\title{
Wiring and information processing in the olfactory bulb of larval Xenopus laevis
}

\author{
Dissertation \\ for the award of the degree \\ "Doctor rerum naturalium" \\ of the Georg-August-Universität Göttingen
}

within the doctoral program International Max Planck Research School (IMPRS) for Neurosciences

of the Georg-August University School of Science (GAUSS)

submitted by

Thomas Offner

from Nürnberg

Göttingen 2019 


\section{Thesis Committee}

Prof. Dr. Ivan Manzini (Reviewer)

Department of Animal Physiology and Molecular Biomedicine, Institute of Animal Physiology, JustusLiebig-Universität, Gießen

Prof. Dr. Thomas Dresbach (Reviewer)

Department of Anatomy and Embryology, Institute of Anatomy and Embryology, University Medical Campus, Göttingen

Prof. Dr. Silvio Rizzoli

Department of Neuro- and Sensory Physiology, Institute of Neuro- and Sensory Physiology, University Medical Campus, Göttingen

\section{Examination Board}

Prof. Dr. Ivan Manzini (Reviewer)

Department of Animal Physiology and Molecular Biomedicine, Institute of Animal Physiology, JustusLiebig-Universität, Gießen

Prof. Dr. Thomas Dresbach (Reviewer)

Department of Anatomy and Embryology, Institute of Anatomy and Embryology, University Medical Campus, Göttingen

\section{Further Members of the Examination Board}

\section{Prof. Dr. Silvio Rizzoli}

Department of Neuro- and Sensory Physiology, Institute of Neuro- and Sensory Physiology, University Medical Campus, Göttingen

Prof. Dr. Jochen Staiger

Department of Neuroanatomy, Institute of Neuroanatomy, University Medical Campus, Göttingen

\section{Prof. Dr. André Fiala}

Department of Molecular Neurobiology of Behavior, Schwann-Schleiden Research Center, Göttingen

\section{Dr. Camin Dean}

Trans-synaptic Signaling Group, European Neuroscience Institute, Göttingen 


\section{Contents}

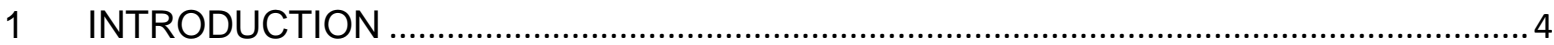

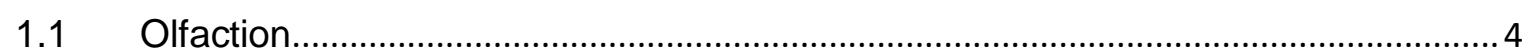

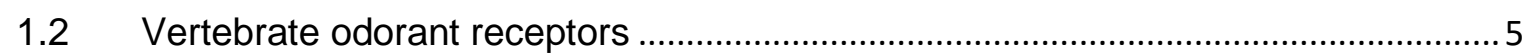

1.3 Molecular structure and ligand selectivity of vertebrate odorant receptors.............. 7

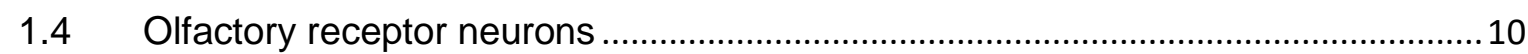

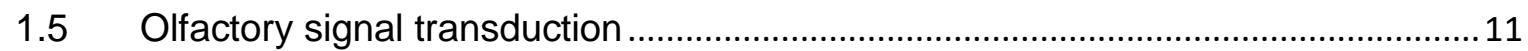

1.6 Olfactory subsystems in vertebrates: functional and anatomical classification.....15

1.7 Odorant receptor expression and ORN axonal wiring logic ..................................19

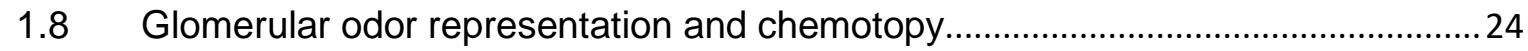

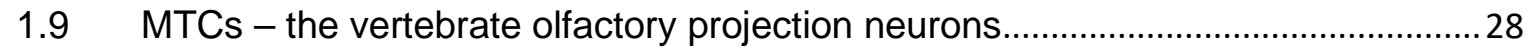

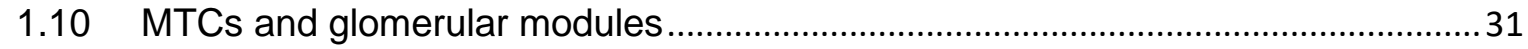

1.11 Output neurons as functionally coupled excitatory modules ..................................32

1.12 Neuromodulation of individual glomerular modules by interneurons in the GL.....32

1.13 Odor information processing in the OB neuronal network ........................................34

1.14 Population coding in MTCs and glomerular modules in the MOS...........................35

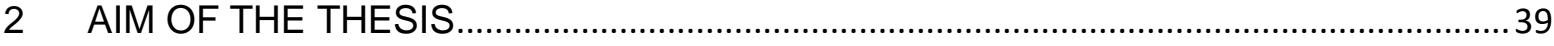

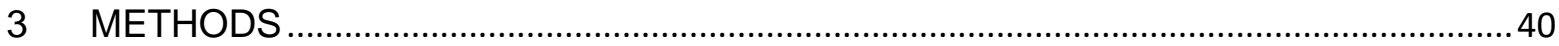

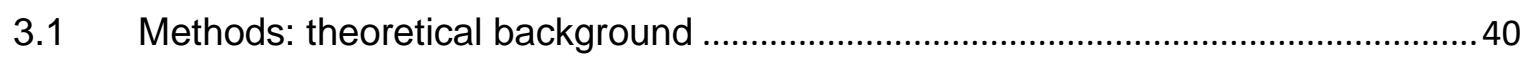

3.1.1 Basics of Fluorescence and Fluorescence Microscopy.....................................40

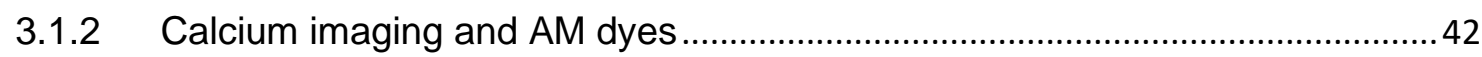

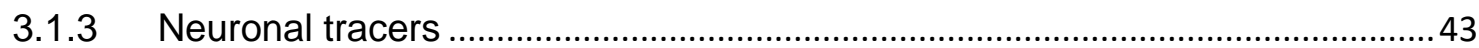

3.1.4 Electroporation of dextran coupled fluorophores into neurons..........................44

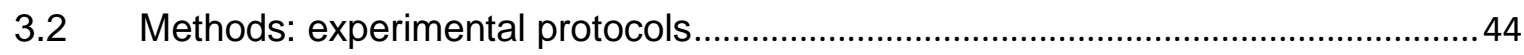

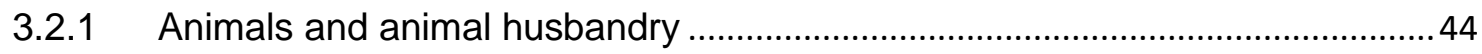

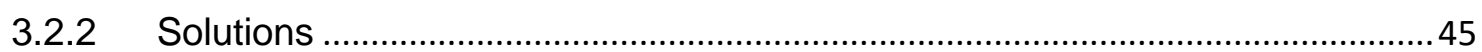

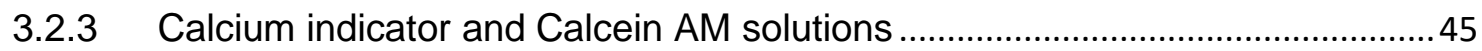

3.2.4 Bulk electroporation of the main olfactory epithelium .........................................45

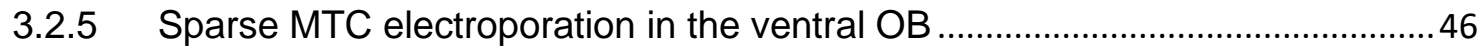

3.2.6 Retrograde tracing of ORNs with WGA-Alexa Fluor conjugates and neuronal

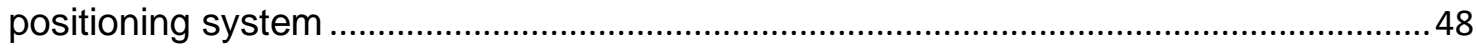

3.2.7 Anterograde labeling of glomerular clusters via dextran coupled WGA ..........50

3.2.8 Whole mount olfactory system preparation for calcium imaging.......................51

3.2.9 Multiple cell bulk loading (MCBL) of AM dyes into the ventrolateral MCL .......51

3.2.10 Multiphoton calcium imaging in the larval olfactory bulb .................................52

3.2.11 Processing and evaluation of calcium imaging data ..........................................54

3.2.12 Analysis and visualization reactive regions to sulfated steroid responses in the

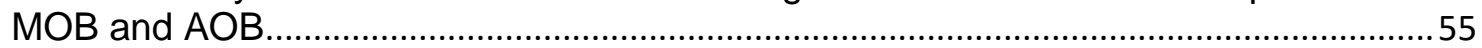

3.2.13 Processing and evaluation of tracings with WGA-coupled fluorophores .........55

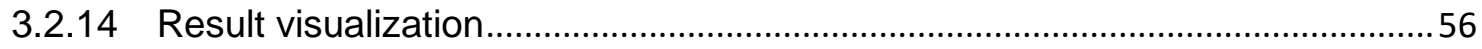


4.1 A ventro-laterally located ORN population innervates the lateral glomerular cluster in larval Xenopus.

4.2 MTCs show topographical association with their neighboring glomerular cluster which is also mirrored in their dendritic and axonal projections.

4.3 Projections of MTCs' apical dendrites are only coarsely topographically arranged within their associated glomerular cluster

4.4 MTCs are very heterogeneous in their individual dendritic configurations but can be classified by number of tufted primary dendrites...

4.5 MTCs with multiple tufts can innervate multiple anatomically distinct glomeruli ...65

4.6 NbT promotor activity labels a subset of MTCs and ORNs in larval Xenopus OB66

4.7 Subsets of NbT positive MTCs show responses to amino acid odorants.

4.8 Glomeruli, JGCs and MTCs preferentially respond to defined groups of amino acids and show differential tuning to amino acids' individual molecular features..... .68

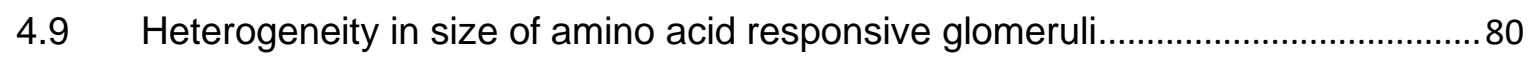

4.10 Lack of stereotypy in the glomerular odor maps between animals ..........................80

4.11 Juxtaposition of glomeruli is not correlated to similarity in odor tuning....................83

4.12 Juxtaposition of NbT-positive MTCs is not correlated to similarity in odor tuning. 85

4.13 Sulfated steroids are suitable stimuli for the MOB and AOB neural network of

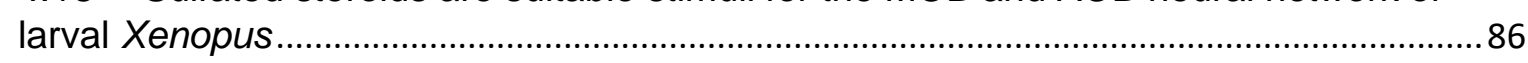

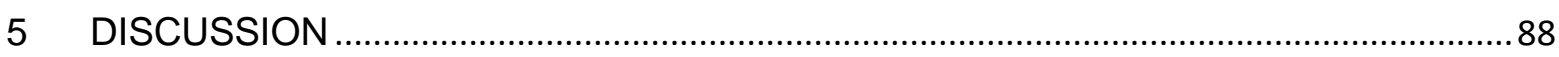

5.1 Zonal distribution of ORNs in MOE and OB of larval Xenopus:...............................8

5.2 partially segregated sub-streams of the LC based on odor groups and VR

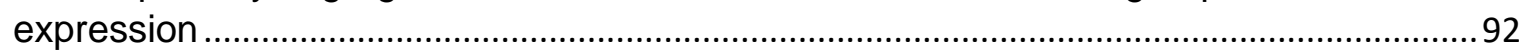

5.3 Odor map organization of the lateral glomerular cluster ..........................................95

5.4 Odor representation in a developing olfactory system ............................................96

5.5 MTCs in larval Xenopus laevis - A morphologically heterogeneous group of neurons.

5.6 Uni- and multi-glomerular MTC wiring patterns as a result of different V2R receptors.

5.7 A 'threaded labeled line' (THRLL) model of glomerular information processing.111

5.8 NbT expression as a marker of a subset of MTCs in larval Xenopus.................... 112

5.9 Odor information processing in the OB neuronal network .....................................116

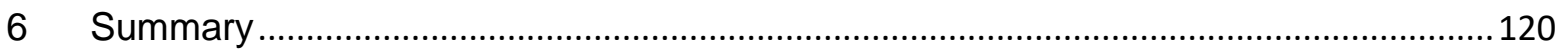

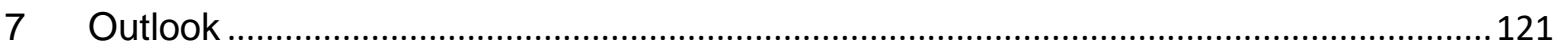

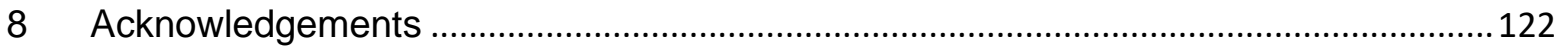

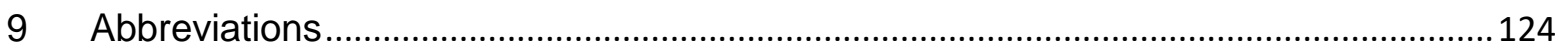

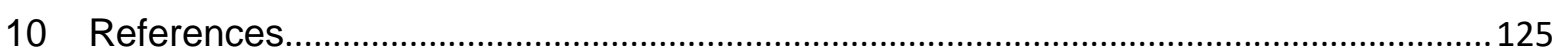

\section{Schematics and Figures}


Schematic 1 Combinatorial receptor coding of odors.

Schematic 2 Odorant receptor dependent signal transduction pathways ...............................14

Schematic 3 ORN subtypes and anatomical segregation of olfactory subsystems in

zebrafish, Xenopus and rodents

Schematic 4 Differential odorant receptor expression patterns in the olfactory subsystems

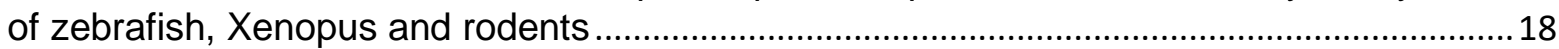

Schematic 5 Axonal wiring in the rodent MOS and glomerular odor representation ..............23

Schematic 6 Differences in odor representation between the rodent MOS and AOS ...........26

Schematic 7 Morphological differences between MTCs of fish, amphibians and rodents....31

Schematic 8 Main cell types of glomerular modules in the rodent MOB ..................................34

Schematic 9 MCs and TCs as parallel odor information processing channels........................36

Schematic 10 Intra- and inter-glomerular neuromodulation in the GL ..................................37

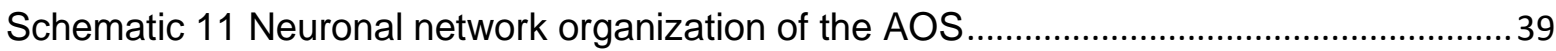

Schematic 12 Principles of epifluorescence, confocal and multiphoton microscopy .............42

Schematic 13 Bulk electroporation of dextran-coupled fluorophores and WGA staining of

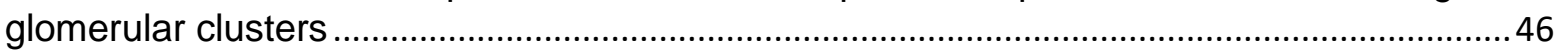

Schematic 14 Sparse cell electroporation and MCBL of Fluo-4 AM calcium indicator..........48

Schematic 15 Multi-color retrograde tracing of ORN axonal projections with WGA-coupled

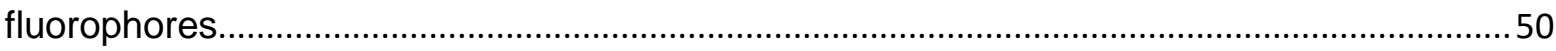

Schematic 16 Labeling of neuronal subpopulations in the OB via Calcein AM dye injections

Figure 1 Ventrolateral distribution of ORNs innervating the lateral glomerular cluster revealed by multicolor retrograde tracing with WGA-coupled fluorophores............................58

Figure 2 Labeling of MTC populations and their dendritic projections associated to the glomerular clusters

Figure 3 Coarse topographic arrangement of MTCs' axonal projections and apical dendrites

61

Figure 4 Morphological staining of MTCs via sparse-cell electroporation in the MCL of larval Xenopus laevis.

Figure 5 MTC morphological subtypes regarding number of tufted primary dendrites and neurite distributions.

Figure 6 Dendritic contributions to glomerular volumes and multi-glomerular sister-MTCs.66

Figure $7 \mathrm{NbT}$ promotor activity and odor responses to amino acid stimuli in NbT-positive

MTCs of the LC.

Figure 8 Selective tuning profiles to single amino acids' structural features in glomeruli and JGCs

Figure 9 Odor response profiles to amino acid stimuli in NbT-positive and negative neurons

Figure 10 Categorization of odor response profiles by their tuning to dominant stimuli and

relative response amplitude differences

Figure 11 Odor tuning and relative differences in response amplitudes of glomeruli and

JGCs to amino acids.

Figure 12 Odor tuning and relative response amplitude differences in NbT-positive MTCs

and $\mathrm{NbT}$-negative neurons of the $\mathrm{MCL}$

Figure 13 Comparison of threshold-based odor tuning with fluorescence intensity difference

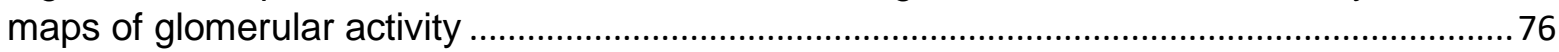

Figure 14 Frequency of the most common odor tunings of glomeruli and JGCs ...................78

Figure 15 Frequency of the most common odor tunings in NbT-positive MTCs and NbT-

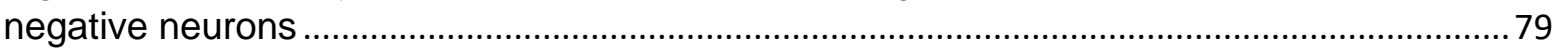

Figure 16 Sizes and spatial distribution of glomeruli and JGCs in the lateral larval OB .......81

Figure 17 Non-stereotypical glomerular odor representation between animals in the LC ....82 
Figure 18 Variable number of glomeruli between animals and dispersed distribution of similary tuned glomeruli in the LC

Figure 19 Quantification of glomerular juxtaposition and similarity in odor tuning .................84

Figure 20 Lack of correlation between distance and similarity in odor tuning among glomeruli or JGCs.

Figure $21 \mathrm{NbT}$-positive MTCs of similar odor tuning are not spatially clustered ....................86

Figure 22 Sulfated steroids are processed in the olfactory bulb (Figure taken from Sansone

et al., 2015, Figure 4; made by Thomas Hassenklöver; own published results) ....................87

Schematic 17 The ventrolateral olfactory stream in larval Xenopus laevis and its subdivision on the glomerular and MTC level.

Schematic 18 Distribution of glomerular reactivity to different odorant groups among the LC lobes and the AOB

Schematic 19 MTC glomerular wiring strategies and possible odorant receptors involved 108 Schematic 20 ORN axonal wiring strategies and possible odorant receptors involved ......110 Schematic 21 Possible co-existence of different wiring strategies in the LC of larval Xenopus

Schematic $22 \mathrm{NbT}$ expression as a possible marker for immature MTCs in a developing olfactory subsystem

Schematic $23 \mathrm{NbT}$ expression as possible marker distinguishing mature MTCs from a rodent AOS-like TC type

\section{INTRODUCTION}

\subsection{OLFACTION}

All life on earth lives at least partially submerged in aqueous waterbodies or surrounded by the gaseous atmosphere of the planet. Every moment, myriads of molecules from both living and inorganic matter dissipate to a small extent into the respective surrounding media. The sense of olfaction represents the ability of animals to perceive fractions of this plethora of molecules. Olfactory sensory systems emerged early in evolution and have proven to be incredibly adaptive during life's conquest of both water and land. This adaptability is, to a considerable part, achieved by the numerous odorant receptors that have evolved to detect structural features of molecules. In addition to the multitude of odorant receptors, vertebrate olfactory systems have developed several neuronal wiring strategies to encode odor information in elaborate neural networks. Amphibians like the African clawed frog Xenopus laevis offer the unique opportunity to investigate the olfactory system of vertebrates that live in the transition zone between water and land. Understanding how the amphibian olfactory systems wires and processes odor information given its odorant receptor repertoire, can thus give invaluable insights into vertebrate olfaction on a bigger scale. 


\subsection{VERTEBRATE ODORANT RECEPTORS}

The discovery of the olfactory receptor (OR) gene family by Buck and Axel (1991) was the hour of birth of modern olfactory neuroscience. The multigene family of $G$ protein-coupled, seven-transmembrane receptors have not ceased to contribute to our understanding of the sense of smell until now. More than 25 years after their discovery, the initial OR genes are currently in the company of a handful of other odorant receptor gene families. Taken together, they represent the fundamental basis of olfaction across vertebrate species (Bear et al., 2016; Silva and Antunes, 2017). Since this work centers around the vertebrate olfactory system, I will mainly address vertebrate odorant receptor families most important in fish, amphibians, and rodents.

The odorant receptor families in rodents can be subdivided by their gene sequences and molecular structures into the OR-type odorant receptors (ORs; Buck and Axel, 1991); vomeronasal type 1 receptors (V1Rs; Dulac and Axel, 1995), vomeronasal type 2 receptors (V2Rs; Herrada and Dulac, 1997; Matsunami and Buck, 1997; Ryba and Tirindelli, 1997), trace amine associated receptors (TAARs; Liberles and Buck, 2006), Formyl-PeptideReceptors (Liberles et al., 2009; Rivière et al., 2009) and MS4A receptors (Greer et al., 2016). Since they were the first odorant receptor genes to be discovered, OR genes, and thus their receptors, are often referred to as the canonical odorant receptors (Buck and Axel, 1991). The multigene family of ORs in rodents covers more than 1000 different genes and thus represents the biggest clade of odorant receptors in the olfactory system (Buck and Axel, 1991; Zhang and Firestein, 2002; Zhang et al., 2004). In fact, the seven-transmembrane G protein-coupled receptors (GPCRs) are the largest multigene family of the entire mammalian genome, exceeding the number of all other class-A GPCRs taken together (Buck and Axel, 1991; Mombaerts, 2004a; Zhang and Firestein, 2002; Zhang et al., 2004). ORs can be subdivided into two major classes according to their amino acid sequence homology: The OR type I and the OR type II receptors (Niimura and Nei, 2005a, 2007; Zhang et al., 2004). The OR type I class makes up around 10-20 \% of the functional OR genes (Niimura and Nei, 2007). Vomeronasal type-1 receptors (V1Rs) represent another type of GPCR that was named after its discovery in rat vomeronasal receptor neurons (VRNs). While they share the seven-transmembrane, rhodopsin-like topology of ORs, they are not homologous in their amino acid sequence to the latter (Dulac and Axel, 1995). The second type of VRN odorant receptors are the vomeronasal type-2 receptors (V2Rs) encoded by the Vnmr2 gene family (Dulac and Axel, 1995; Herrada and Dulac, 1997; Matsunami and Buck, 1997; Ryba and Tirindelli, 1997). The 120 potentially functional Vnmr2 genes (Young and Trask, 2007) differ significantly in sequence compared to the Vnmr1 family (Dulac and Axel, 1995; Herrada and Dulac, 1997). The V2Rs have a different topology than V1Rs and show high sequence variability inside their clade. Most V2Rs possess large hydrophobic $\mathrm{N}$-terminal ligand binding domains and display similarity with metabotropic glutamate receptors. However, a few of 
them resemble taste receptors or calcium-sensing receptors (Dulac and Torello, 2003; Mombaerts, 2004a; Spehr and Munger, 2009). Formyl-peptide receptors proteins represent a family of classical seven-transmembrane GPCR receptors first found in cells of the innate immune system (Le et al., 2002) A subset of the formyl-peptide-receptor family (Fpr-rs 3, 4, $6,7)$ acts as exclusive chemosensors in VRNs (Liberles et al., 2009; Rivière et al., 2009). Conclusions drawn from the one atypical member of the mammalian trace-amine-associated receptors (TAAR1) family, led to the assumption that TAARs are generally detectors of trace amines, a rare form of neurotransmitter (Borowsky et al., 2001; Lindemann and Hoener, 2005; Lindemann et al., 2005). However, other TAARS investigated are expressed exclusively in the olfactory system (MOS only) and fulfill all requirements for odorant receptors (Liberles and Buck, 2006). While they are rather few in numbers in rodents (15 in the mouse; 17 in rat), their orthologous genes in teleost fish can be in the range of more than a hundred members (zebrafish, Korsching, 2008). MS4A receptors are the most recent group of chemoreceptors found to serve as putative odorant receptors. A subset of the MS4A family is associated with the necklace glomeruli subsystem. Its exact function and biological relevance have yet to be revealed (Greer et al., 2016).

While most odorant receptor families have been discovered in rodents, it is important to take into account that from the evolutionary perspective, odorant receptors emerged in fish first. In this work, I will focus mainly on the teleost odorant receptor gene families. It needs to be noted, though, that the common ancestor of odorant receptors (f. ex ORs) might be traced back before the divergence of jawed and jawless fish (Freitag et al., 1999). Teleost fishes like the zebrafish Danio rerio are a suitable model for aquatic olfaction, not only from the molecular biology (Sprague et al., 2006) but also from the evolutionary perspective (Korsching, 2008). Zebrafish is a more 'primitive' species than other studied neo-teleost fish like Medaka and pufferfish. Consequently, it is better suited to compare odorant receptor gene evolution between fish and tetrapods (Korsching, 2008) The OR family of zebrafish comprises around 100 functional genes (Alioto and Ngai, 2005; Niimura and Nei, 2005b). A high divergence in sequence can be observed among the receptors of the teleost OR family (Ngai et al., 1993). The ORA family of zebrafish are homologous to the mammalian V1R receptor genes (Pfister and Rodriguez, 2005; Saraiva and Korsching, 2007; Shi and Zhang, 2007). The seven-transmembrane receptors belonging to GPCR class-A are highly conserved among teleost fish. In zebrafish, six members have been characterized so far (Saraiva and Korsching, 2007 OlfC receptors are class C GPCRs and in terms of their molecular topology related to the phylogenetically younger mammalian V2Rs. However, zebrafish OlfCc genes form a phylogenetically distinct clade, that might have emerged from an ancestral calcium-sensing protein family (Alioto and Ngai, 2006). According to recent findings, OlfCc receptors are unique in their biological function and might not be putative odorant receptors in the canonical sense. (DeMaria et al., 2013; Sato et al., 2005; Speca et al., 1999). While other receptor gene classes outnumber TAAR genes in the mammalian 
olfactory system, they are abundant in teleost fish (Hashiguchi and Nishida, 2007). The zebrafish TAAR repertoire covers 109 genes, which is considerably bigger than the repertoires of other investigated neo-teleost fish like medaka and stickleback (Hashiguchi and Nishida, 2007).

Amphibians like the African clawed frog Xenopus laevis offer an excellent opportunity to investigate odorant receptor evolution on a comparative level (Bear et al., 2016; Silva and Antunes, 2017). Most amphibians spend at least their early development in aquatic environments and can, in the course of their lives, pursue a variable extent of terrestrial lifestyles that require olfaction (Woodley, 2014). Depending on the developmental stage and particular lifestyle, their odorant receptor repertoire needs to be capable of potentially detecting airborne odorants in addition to waterborne odorants. The size and composition of the odorant receptor repertoire often mirror the animal's lifestyle (Shi and Zhang, 2007; Silva and Antunes, 2017). Xenopus laevis is a secondarily aquatic frog adapted to an almost exclusive aquatic lifestyle. The adult animals possess a water-nose and an air-nose (Altner, 1962; Dittrich et al., 2016; Hansen et al., 1998). Their odorant receptor repertoire mirrors their secondary aquatic lifestyle and their intermediate status between fish and terrestrial vertebrates: Xenopus laevis possesses hundreds of OR-type odorant receptor genes composed of both 'fish-like' type I and 'mammalian-like' type II ORs (Freitag et al., 1995; Mezler et al., 1999; Niimura and Nei, 2005b; Saraiva and Korsching, 2007; Shi and Zhang, 2007). Their vomeronasal receptor (VR) repertoire outnumbers even the number of VRs in rodents (Niimura and Nei, 2005a). OR- and VR-type odorant receptors are expressed in a defined spatio-temporal pattern throughout the development and metamorphosis of Xenopus laevis that will be addressed in section 1.6 (Gliem et al., 2013; Mezler et al., 1999; Syed et al., 2013, 2017). The gene family of V2Rs in Xenopus tropicalis covers several hundred receptors and is composed of more 'fish-like 'and some phylogenetically younger 'mammalian-like' odorant receptor types. The VR subfamilies are differentially expressed among the olfactory sensory surfaces (Date-Ito et al., 2008; Hagino-Yamagishi et al., 2004; Syed et al., 2013). From the five known Xenopus laevis TAAR genes, the expression of two of them (TAAR4a and TAAR4b) has been validated in the olfactory mucosa (Hussain et al., 2009; Syed et al., 2015).

\subsection{MoleCULAR STRUCTURE AND LigAND SELECTIVITY OF VERTEBRATE ODORANT RECEPTORS}

Odorant receptors are the basic functional units enabling an organism to detect molecules of its external world. The entity of molecules that can be detected by the odorant receptors of an organism defines its hypothetical odor space (Korsching, 2001). Each odorant receptor thus represents a singular information channel that encodes the short-lived interactions of odorant molecules with the binding pocket of the receptor protein. Consequently, the animal's 
odorant receptor repertoire and each receptor's specific odorant binding pocket defines the perceivable odor space et (Korsching, 2001).

ORs belong to the family of the seven-transmembrane, opsin-like class-A GPCRs (Buck and Axel, 1991). Studies of light-induced conformational changes in rhodopsin and adrenergic receptors (Ballesteros and Palczewski, 2001; Farrens et al., 1996) contributed to biophysical models of OR conformational changes. In contrast to adrenergic receptors in which ligand interactions are stabilized mostly due to ionic bonds, odor molecules typically show weak interactions, with the hydrophobic OR binding pocket (Kato et al., 2008). These weak interactions are important to understand the selective but also broad binding properties of ORs. While the pocket is sterically specific for each OR, the binding interaction can occur with different affinities for a range of molecules that share similar molecular epitopes (Katada et al., 2005). These binding properties of ORs are the biophysical correlate for the combinatorial receptor coding in odor detection (Malnic et al., 1999). The immense variety of OR binding pockets explains crucial features of odor detection: First, individual odorant receptors can be activated by a large number of molecules capable of interacting with its binding pocket. Second, each odorant can potentially be detected by several ORs depending on its molecular epitopes (Malnic et al., 1999). This strategy provides an economical way of potentially encoding for both single as well as complex odors by unique fingerprints of activity (Schematic 1).

In contrast to the singular information channels of the visual system (colors), the odor representation created by OR activity overlaps and cannot easily be separated into modalities (Korsching, 2001). Moreover, higher concentrations of odorants in proximity to the ORs increase the probability of odorants molecules binding to additional less specific OR species. This concentration dependence of odor representations (Fried et al., 2002; Meister and Bonhoeffer, 2001) is a fundamental challenge to be accomplished by the olfactory neuronal network and will be discussed in more detail later on.

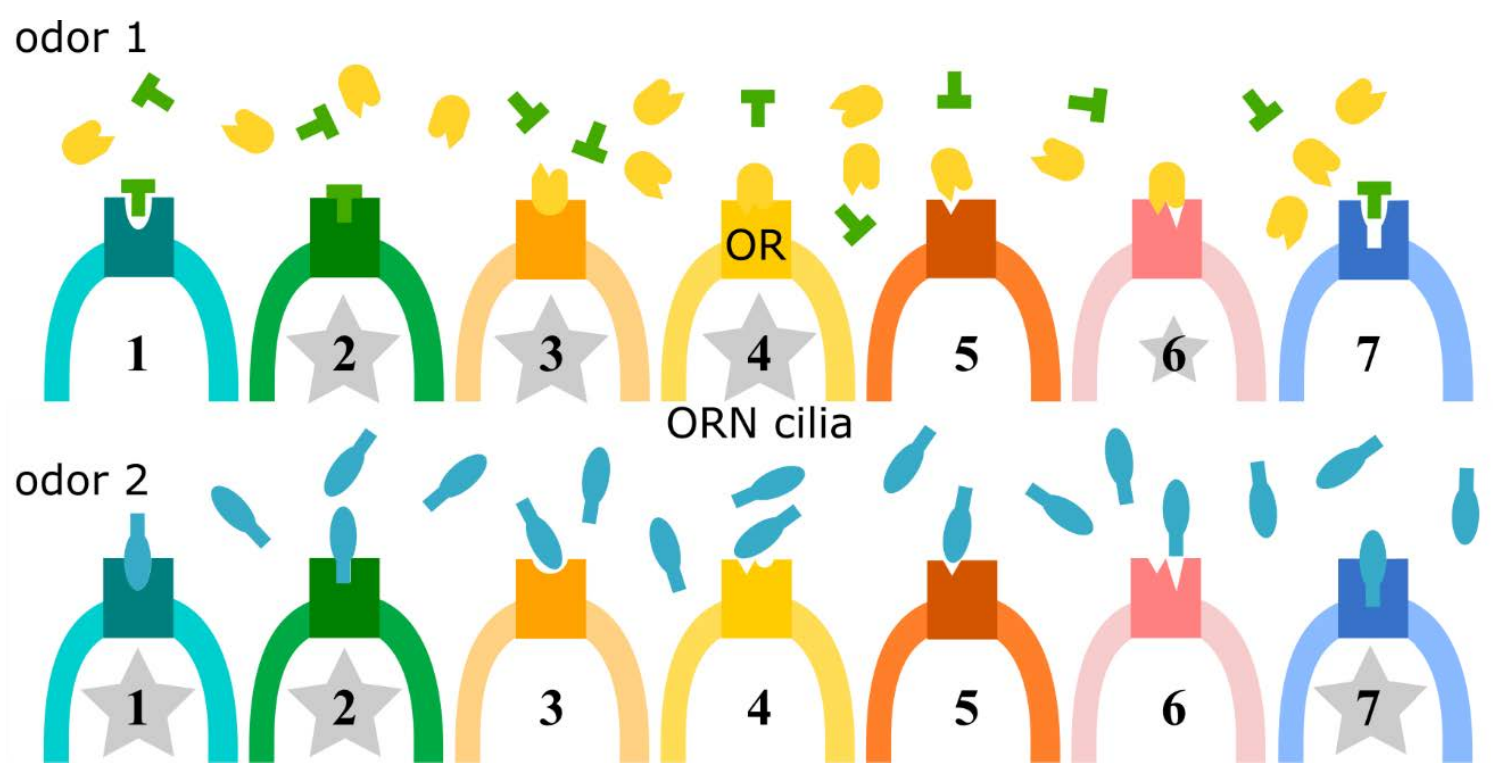


Odors consisting of multiple (odor 1, yellow and green shapes) or single molecules (odor 2, blue shapes) can activate subsets of ORNs expressing individual odorant receptors (f.ex. ORs) on their cilia (different colors; numbers: 1-7). Molecular epitopes of the odorant molecules bind to ORs and activate subsets of ORN species (numbers, grey stars) in a combinatorial manner (odor 1 activates ORN species 2, 3, 4 and 6, odor 2 activates ORN species 1, 2 and 7). Different affinity binding (odor 1: ORN species 2,3 vs. 6) as well as overlapping activity patterns (ORN species 2 activation by both odor 1 and odor 2) are fundamental features of combinatorial receptor coding

While ORs are suitable to detect mostly small, volatile airborne odorants (Matsumoto et al., 2010), VRs operate in the aqueous medium. Aqueous, viscous mucus fills the inner lumen of the VNO, which represents the medium VRs operate in (Meredith, 1991). Comparably few suitable odorants are known. Most compounds have been characterized as water-soluble, non-volatile, macromolecules, especially peptides and proteins (Leinders-Zufall et al., 2004). V1Rs resemble ORs in their molecular topology (Dulac and Axel, 1995) and are mostly associated with the detection of a heterogeneous group of small molecules, like putative pheromones or modified steroid derivatives (Nodari et al., 2008). Sulfated steroids can induce odorant responses in V1R-expressing VRNs already at nanomolar concentrations (Turaga and Holy, 2012). The ancestral V1R-like ORA receptor family is present and highly conserved among fish investigated so far (Pfister and Rodriguez, 2005; Saraiva and Korsching, 2007; Shi and Zhang, 2007). The binding pocket and ligands for the six members of the zebrafish ORA family have recently been described (Cong et al., 2019). ORAs exhibit highly selective but combinatorial binding affinity to steroid derivates like bile acids or bile salts. A conserved 'anchor' for the acid group was described in addition to the steroid selective but variable pocket (Cong et al., 2019). In Xenopus tropicalis, the V1R family covers a group of 21 putative odorant receptors (Date-Ito et al., 2008; Shi and Zhang, 2007), of which four have been validated to be expressed in Xenopus laevis (Gliem et al., 2013). No odorant ligands are known yet for the receptor family so far to the best of my knowledge.

The binding of odorants to V2Rs is not as well characterized but should involve the large characteristic $\mathrm{N}$-terminal domain of the receptor family (Mombaerts, 2004a). ). The lack of known odorants has been interpreted as a sign of higher selectivity of V2Rs (Kimoto et al., 2005; Leinders-Zufall et al., 2009). To fully comprehend the individual tuning profiles of VRNs, the non-random co-expression of vmn $2 r(A, B$ and $D)$ and single selected $v m n 2 r$ receptors needs to be taken into account (Ishii and Mombaerts, 2011; Silvotti et al., 2007). The V2R family is of particular importance for this work since it might be essential to understand odorant responses in aquatic animals to single amino acids (Alioto and Ngai, 2006; DeMaria et al., 2013; Syed et al., 2013). Structurally related proteins imply that OlfC receptors (the fish analog of V2R-Cs) possess a selective binding pocket for amino acid or amino acid-related peptides and derivates (Acher and Bertrand, 2005; Alioto and Ngai, 2006; Bertrand et al., 2002). OlfC receptor ligands and exact binding mechanisms are very elusive to date. One of the well-investigated OlfC receptors in zebrafish, OlfC-c1 seems to play a unique role in OlfC 
odorant recognition. While having an intrinsic tuning for amino acids, the OlfCc1 receptor can serve as chaperones and co-receptors for other OlfC receptors (DeMaria et al., 2013). They were shown to be essential for amino acid detection in zebrafish (DeMaria et al., 2013). While concrete evidence is still missing, members of the amphibian V2R family are likely expressed in a subset of amino-acid sensitive receptor neurons in Xenopus laevis (Gliem et al., 2013; Syed et al., 2013). Whether the broad tuning of receptor neurons to amino acids in Xenopus laevis is a direct result of $\mathrm{v} 2 \mathrm{r}-\mathrm{C}$ expression similar to OlfCc1 in zebrafish remains unknown still (DeMaria et al., 2013; Gliem et al., 2013; Schild and Manzini, 2004).

In non-olfactory research, studies have well described the family of TAARs and their binding properties on biogenic amines (Shi and Javitch, 2002). A characteristic aspartate-residue forms ionic interactions with the amine-group bearing ligands (Lindemann and Hoener, 2005). The olfactory TAARs detect small amines and are thus quite selective in comparison to canonical ORs while displaying similar ligand affinities (Liberles and Buck, 2006). The high abundance of functional TAAR genes in teleost fish like zebrafish highlights their importance in aquatic olfaction (Saraiva and Korsching, 2007). By evolutionary changes in the arrangement or number of aspartate residues contributing to the binding pockets of different TAARs, neo-teleost fish like zebrafish obtained the possibility of detecting a broader range of biogenic amines and the possibility to discriminate between mono- and diamines ( $\mathrm{Li}$ et al., 2015). In Xenopus laevis, TAARs have also been proposed as candidates for the detection of amines. Comparing the bimodal distribution of two TAARs and odorant responses to amines in the olfactory mucosa, Syed and colleagues ruled in the possibility of several coexisting amine detecting systems and receptors in amphibians (Syed et al., 2015).

\subsection{OLFACTORY RECEPTOR NEURONS}

The olfactory receptor neuron (ORN) in vertebrates is a bipolar neuron. It exhibits a knobbed dendrite bearing tubular membrane specializations and an axon projecting from its opposite pole (Figueres-Oñate et al., 2014; Schultze, 1856). The odorant receptors are usually expressed on the dendritic membrane specializations, where they lie exposed to the external world and create electrical potentials upon odor activation (Ottoson, 1956). The axons of the ORNs project to their particular target regions in the brain where they confer the odor information (Mombaerts, 2006; Mombaerts et al., 1996). This basic blueprint applies to most ORNs even though morphological variety can be observed across individual species (Hamdani and Døving, 2007; Manzini and Korsching, 2011; Schild and Restrepo, 1998).

In mammals, there are two major morphological types of ORNs. They either bear immotile cilia or microvilli as specializations on their dendritic knob (Figueres-Oñate et al., 2014; Schild and Restrepo, 1998). The microvillous ORNs of rodents reside in the vomeronasal organ (VNO) and are called vomeronasal receptor neurons (VRNs; Meredith, 1991; Trotier and Døving, 1998). ORs and TAARs are expressed in ciliated ORNs of the MOS (Munger et 
al., 2009). Members of the V1R, V2R and FPR multigene families are usually expressed by subgroups of microvillous VRNs in the VNO (Munger et al., 2009).

In most teleost species investigated, microvillous and ciliated ORN types are present (Hamdani and Døving, 2002; Hamdani et al., 2001; Hansen et al., 2003; Morita and Finger, 1998; Sato et al., 2005). The majority of ciliated ORNs express OR-type receptors and TAARs whereas ORA (V1R-like) and OlfC (V2R-like) expression is mostly confined to microvillous ORNs (Cao et al., 1998; Hansen et al., 2004; Hussain et al., 2009; Korsching, 2008; Speca et al., 1999). Three additional ORN types exist in zebrafish that I will not discuss in detail in this thesis: the crypt neurons, Kappe neurons and the so-called 'pear-shaped' neuron. They appear to be particular specialized subtypes varying in morphology, signal transduction and receptor expression compared to the big two groups of ciliated and microvillous ORNs (crypt neurons: (Hamdani and Døving, 2007; Hansen and Finger, 2000; Oka et al., 2012); Kappe neuron: (Ahuja et al., 2014); 'pear-shaped': (Wakisaka et al., 2017)).

Both ciliated and microvillous ORNs exist in larval and adult Xenopus laevis (Gliem et al., 2013; Hansen et al., 1998; Nakamuta et al., 2011). Several studies imply that a big part of Xenopus laevis microvillous ORNs express VRs. To be more precise, the VRNs of the VNO express V2Rs but no V1Rs. The latter are solely expressed in the main system (Freitag et al., 1995; Mezler et al., 1999). V2Rs are expressed in the main system, more precisely, in the basal ORN population (Syed et al., 2013). The special v2r-2 C receptors showed broad and overlapping expression with a big part of the microvillous ORN population in larval Xenopus laevis (Syed et al., 2013). Consequently, V2R expression seems to be linked to a subset of microvillous ORNs. During the metamorphic remodeling of the olfactory organ, V2R expression shifts together with the respective ORN population to the newly formed waternose (Syed et al., 2017). In parallel, V2R expression ceases in the former larval water-nose that is remodeled to an air-nose (Dittrich et al., 2016; Syed et al., 2017). Whether there is a clear co-expression of $v 2 r$ type receptors of families $(A, B, D)$ with one family $C$ receptor as in the VNO of rodents (Ishii and Mombaerts, 2011; Silvotti et al., 2007) is not clear yet.

\subsection{OLFACTORY SIGNAL TRANSDUCTION}

\section{'Canonical', cAMP dependent olfactory signal transduction cascade in rodents}

Odorant receptors can be found expressed on cilia or microvilli of OSNs (Ottoson, 1956). Independent of the receptor family, the basic molecular transduction machinery in the majority of ORNs consists of four principal (macro-)molecular complexes: a receptor protein, a heterotrimeric G-protein, a membrane-associated enzyme and a set of ion channels (Schematic 1). Already before the OR gene family was discovered, specific mechanisms of odor detection and parts of the signal transduction machinery had already been known (Schild and Restrepo, 1998). The G-protein $\mathrm{G} \alpha_{\text {olf }}$ was the first identified $\mathrm{G}$ protein of olfactory transduction. Its absence leads to an anosmic phenotype in mice (Belluscio et al., 1998; 
Jones and Reed, 1989). In further knockout models, the essential roles of adenylate cyclase III (ACIII) and a cyclic-nucleotide gated channel in ORNs were confirmed (Baker et al., 1999; Brunet et al., 1996; Wong et al., 2000). The latter results and pioneering physiological experiments (Firestein et al., 1991; Nakamura and Gold, 1987), solidified the cAMPdependent olfactory transduction pathway associated with the OR family (Firestein et al., 1991; Nakamura and Gold, 1987). Upon odorant binding to ORs, the GTP bound dissociated $\mathrm{G} \alpha_{\text {olf }}$ subunit binds to adenylate-cyclase III initiating the conversion from ATP to second messenger CAMP (Bakalyar and Reed, 1990; Pace et al., 1985). The second messenger cAMP subsequently triggers the opening of cyclic-nucleotide gated cation channels, causing an influx of sodium and calcium into the ORN (Nakamura and Gold, 1987; Firestein et al., 1991; Lowe and Gold, 1993). The increase in calcium conductance was shown to be essential to trigger further amplification of the generator potential by a $\mathrm{Ca}^{2+}$-activated chloride channels (Kleene and Gesteland, 1991; Kurahashi and Yau, 1993; Lowe and Gold, 1993). The resulting $\mathrm{Ca}^{2+}$-activated chloride ion net efflux depolarizes the cell towards its reversal potential at around $0 \mathrm{mV}$ (Kurahashi and Yau, 1993). The resulting net depolarization is sufficiently more positive than the resting membrane potential to trigger action potentials, the unitary electrical events encoding odor information.

\section{'Non-canonical', cAMP independent olfactory transduction cascade in rodents}

Olfactory signal transduction pathways independent of CAMP were already postulated and partially described early in vertebrates like fish and amphibians (Schild and Restrepo, 1998). With the emergence of the VR family and the investigation of VRN signal transduction, the CAMP independent transduction pathway was back on the radar in rodents. The mutually exclusive expression of two heterotrimeric $\mathrm{G}$ proteins ( $\mathrm{G} \alpha_{0}$ and $\mathrm{G} \alpha_{\mathrm{i} 2}$ ) in two distinct subsets of VRNs were first hints towards the essential cAMP-independent transduction cascades in VRs (Halpern et al., 1995). While $\mathrm{Ga}_{0}$ has been confirmed to act in V2R mediated signal transduction in basal VRNs (Chamero et al., 2011), the role of $\mathrm{G} a_{\mathrm{i} 2}$ in V1R transduction has yet to be validated. In addition to the soluble $\mathrm{G} \alpha$ subunit, the membrane tethered ßy-subunits contribute to the heterogeneity of signal transduction cascades (Rünnenburger et al., 2002; Ryba and Tirindelli, 1995; Sathyanesan et al., 2013; Wu et al., 1996). Instead of the adenylate-cyclase, phopholipase C (PLC) serves as a major effector enzyme that produces second messenger molecules. The membrane-associated PLC is activated by binding to the freed ßy subunit upon GDP-GTP exchange in the Ga subunit (Rünnenburger et al., 2002). PLC catalyzes the hydrolysis of phosphatidylinositol-4,5-bis-phosphate (PIP2) in the inner plasma membrane leaflet, setting free membrane-bound diacylglycerol (DAG) and watersoluble inositol-1,4,5-trisphosphate ( $\mathrm{IP}_{3}$; Holy, 2000; Lucas et al., 2003; Rünnenburger et al., 2002; Spehr et al., 2002). The local elevation of second messengers leads to calcium influx via two main mechanisms: $\mathrm{IP}_{3}$ dissipates from the membrane and triggers calcium influx into the cytosol from intracellular compartments (Kim et al., 2011; Yang and Delay, 2010). DAG diffuses laterally through the plasma-membrane of the VRN microvilli and activates TRPC2, 
a member of the transient receptor potential channel family upon binding (Liman et al., 1999; Lucas et al., 2003; Spehr and Munger, 2009). The increased cation conductance of activated TRPC2 leads to further calcium level elevation in the microvilli of VRNs. Subsequently, calcium ions trigger Ca2+-activated chloride net efflux through ANO1or ANO2 chloride channels (Amjad et al., 2015; Dibattista et al., 2012; Kim et al., 2011; Lucas et al., 2003; Münch et al., 2018; Yang and Delay, 2010). Depolarization through ANO1/2 is possible due to the high chloride concentration in VRN microvilli (Kim et al., 2015; Untiet et al., 2016). TAAR receptor transduction is not studied in detail yet. The receptor topology and involvement of $\mathrm{Ga}_{\text {off }}$ in its signal transduction point to a canonical OR like signal transduction cascade (Liberles and Buck, 2006). FPRs (Liberles et al., 2009; Rivière et al., 2009) and MS4A receptors (Greer et al., 2016) will not be discussed further on the signal transduction level in this work.

\section{Signal transduction in aquatic species}

The existence of cAMP dependent and independent signal transduction pathways in fish was already confirmed early on in catfish ORN cilia (Restrepo et al., 1993). While several studies in fish have contributed to the understanding of signal transduction, few of them were able to characterize the two pathways as detailed and convincing as Sato and co-workers (Sato et al., 2005). Analogous to their mammalian counterparts, ciliated ORNs in zebrafish express $G \alpha_{\text {olf }}$ and a cyclic nucleotide gated channel subunit (here CGNA2), main components of the canonical cAMP transduction pathway (Hansen et al., 2003; Sato et al., 2005). The microvillous ORN type was distinguishable from the ciliated type by its expression of TRPC2 (Munger et al., 2009; Sato et al., 2005).

Both cAMP-dependent and independent signal transduction pathways are present in amphibians (Schild and Restrepo, 1998). Signal transduction independent of cAMP has been confirmed in Xenopus laevis ORNs (Manzini and Schild, 2003a). Key players of the cAMPindependent signal transduction cascade including PLC, DAG and TRPC2 were found to be associated with the microvillous ORN type of the larval olfactory mucosa (Gliem et al., 2013; Manzini and Schild, 2003a; Sansone et al., 2014a, 2014b). 

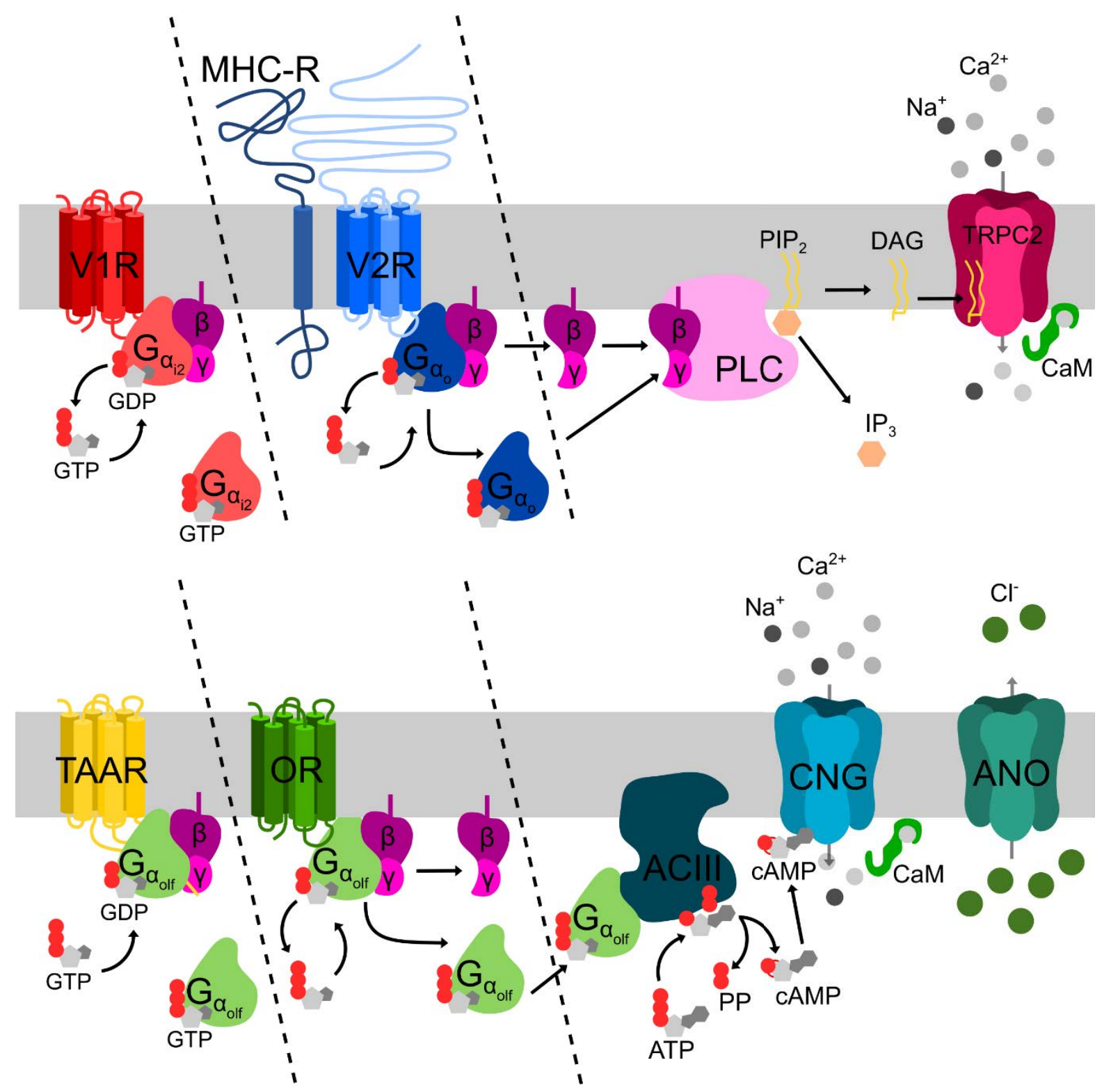

Schematic 2 Odorant receptor dependent signal transduction pathways

Vertebrate odorant receptor classes (V1Rs, V2Rs, TAARs and ORs) use heterotrimeric G-proteins (soluble $\mathrm{G}_{\alpha^{-}}$and membrane tethered ßy-subunit) to transduce information from the exterior to the inside of the cell. Upon odorant binding the odorant receptor undergoes conformational changes that lead to exchange of bound GDP with GTP in the G-protein and its subsequent dissociation into the respective $G_{\alpha^{-}}$(V1R: Gai2, V2R: Gao, TAAR \& OR: Gaolf) and By-subunits. In the V1R and V2R transduction cascade the ßy-subunit, activates PLC which creates the second messengers $\mathrm{IP}_{3}$ and DAG from $\mathrm{PIP}_{2}$. DAG triggers cation-conducting TRPC2 channels, depolarizing the neuron. In the OR and TAAR signal transduction cascade, the GTP-bound Gaolf subunit activates ACIII which catalyzes CAMP production from ATP. Cation influx through CNG channels is triggered upon cAMP binding and the resulting $\mathrm{Ca}^{2+}$ influx induces ANO-mediated $\mathrm{Cl}^{-}$efflux, boosting depolarization of the neuronal plasma membrane. 


\subsection{OlFACTORY SUBSYSTEMS IN VERTEBRATES: FUNCTIONAL AND ANATOMICAL CLASSIFICATION}

Given the large repertoire of odorant receptors, ORN subtypes and different signaling transduction cascades, the concept of a single olfactory system is definitely outdated (Munger et al., 2009). Very different criteria can classify olfactory subsystems. One of the most modern and robust classifications is the receptor family as the overarching principle for a subsystem. Since individual receptor families are often associated with particular transduction machinery and ORN subtypes, receptor-based classifications are well suited for mechanistic and comparative approaches (Munger et al., 2009). However, it is important to remember that in the end, the biological purpose of the olfactory surface dictates its receptor repertoire and neuronal composition (Bear et al., 2016; Silva and Antunes, 2017). Individual receptor repertoires can vary considerably since the biological demands towards an olfactory (sub)system are tightly linked to the lifestyle of the organism (Bear et al., 2016; Shi and Zhang, 2007; Silva and Antunes, 2017). Biologically relevant odor molecules and the medium they occur in, have evolutionary shaped the distribution of olfactory subsystems and often promoted the development of several anatomically distinct sensory surfaces (Shi and Zhang, 2007).

The rodent olfactory sensory surface is not a singular structure but distributed among several distinct olfactory organs (Breer et al., 2006; Munger et al., 2009). The biggest sensory surface is the main olfactory epithelium (MOE), lining the nasal cavities (Barrios et al., 2014). The vomeronasal organ (VNO) consists of two encapsulated epithelial invaginations at the base of the anterior nasal septum. The VNO is filled with mucus and is connected to the nasal cavities by a narrow duct (Halpern and Martínez-Marcos, 2003). Two additional peripheral subsystems that I will not in detail in this work are the septal organ (Rodolfo-Masera, 1943) and the ganglion of Grüneberg (Grüneberg, 1973). The peripheral organs in combination with their higher brain centers are referred to as the main (MOS) and accessory olfactory system (AOS). MOS and AOS are not only anatomically segregated on the sensory surface level: ORNs of the MOE project their axons to the main olfactory bulb (MOB) and the accessory olfactory bulb (AOB) is targeted by VRN axons (Munger et al., 2009). The two systems are subdivided into anatomically segregated territories but do not represent entirely independent systems (Scalia and Winans, 1975; Wilson and Raisman, 1980) 


\section{Zebrafish olfactory mucosa}

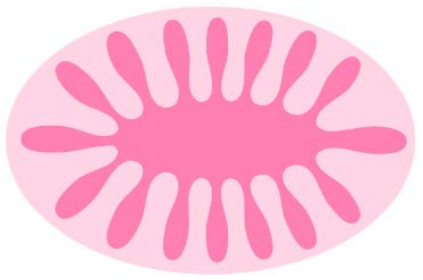

Xenopus larval olfactory organs

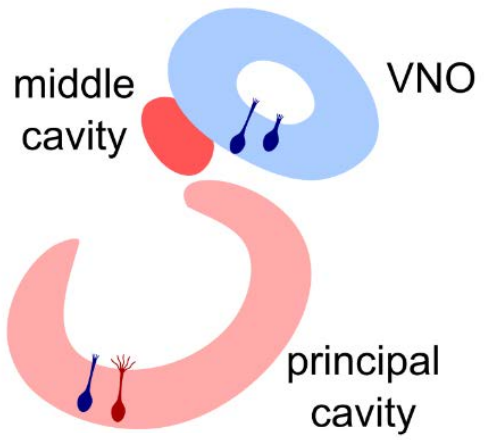

Xenopus adult olfactory organs

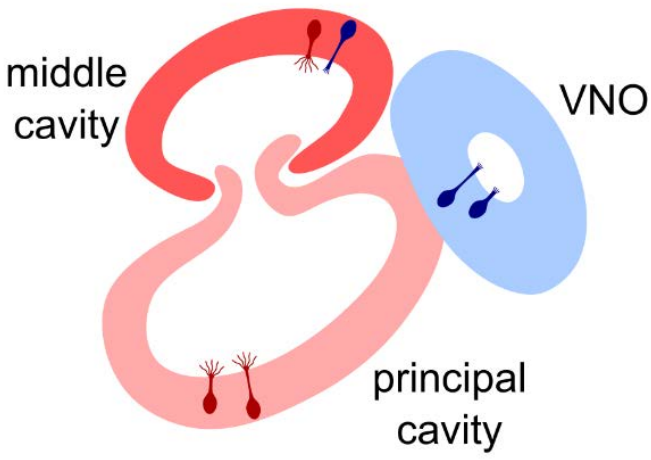

microvillous ORNs

ciliated ORNs

crypt neurons

kappe neurons

pear-shaped neurons

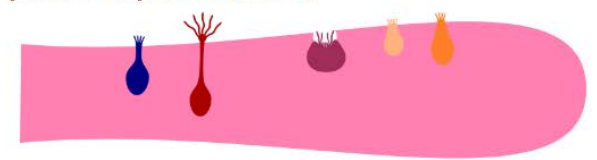

Rodent MOE

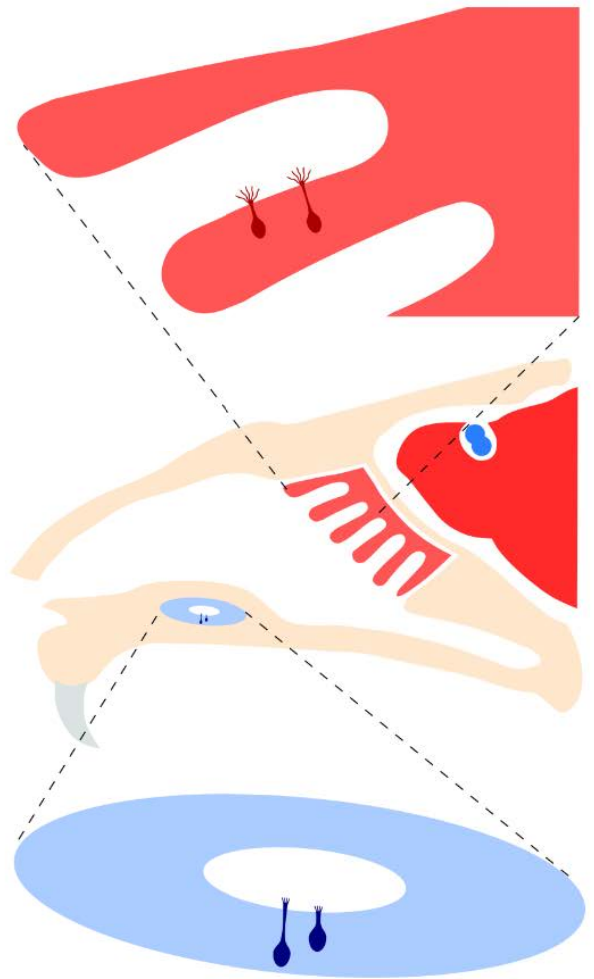

Rodent VNO

Schematic 3 ORN subtypes and anatomical segregation of olfactory subsystems in zebrafish, Xenopus and rodents

In the zebrafish single sensory surface all ORN subtypes exist intermingled in the olfactory mucosa. Individual types (microvillous, ciliated, crypt, Kappe and 'pear-shaped' ORNs) can vary in apical to basal distribution in the mucosa (upper right). In both larval and adult Xenopus there is an anatomical distinct VNO present with microvillous ORNs only. In the water nose (larval principal and adult middle cavity) microvillous and ciliated ORNs coexist. Ciliated ORNs populate the air-nose or principal cavity in adult frogs. The two major olfactory subsystems (MOS and AOS) are anatomically and functionally segregated in rodents. Ciliated neurons inhabit the MOE whereas the VNO is virtually exclusively populated by microvillous VRNs (basal and apical subpopulations).

Rodents represent an 'exceptionally' segregated case regarding the anatomical and functional subdivision into olfactory subsystems (Bear et al., 2016; Munger et al., 2009). While the MOE is predominately composed of ciliated, OR-expressing neurons, the AOS harbors microvillous, cAMP-independent VRNs expressing either V1Rs or V2Rs (Bear et al., 2016; Munger et al., 2009). This virtually exclusive segregation is conserved on both the anatomical and functional levels between the MOS and the AOS (Munger et al., 2009), but see: (Lévai et al., 2006). However, other functional subsystems besides the canonical ciliated ORNs do exist intermingled in the MOS. TAAR, Guanyl-cyclase-D (GC) as well as TRP 
channel expressing ORNs are part of the MOS and exhibit diverse distributions, transduction cascades, and possible biological purposes (Munger et al., 2009).

In contrast to mammalian olfactory systems, the olfactory mucosa of (teleost-) fish represents a singular sensory surface. All types of ORNs and functional subsystems co-exist in this epithelium (Hamdani and Døving, 2007). Differential distributions of ORN types have been observed along the apical-to-basal axis in the individual lamellae of the rosette-shaped olfactory mucosa (Hamdani and Døving, 2007). Microvillous ORNs are found in the apical portion of the epithelium and express V2Rs and the associated cAMP-independent signal transduction cascade described in 1.5 (Hamdani and Døving, 2007; Hansen et al., 2004; Kimoto et al., 2005). Basally located ciliated ORNs represent a cAMP-dependent functional subsystem. Thus, on the microscopic level, a certain anatomical division between the functional subsystems does exits along the apical-basal axis, at least (Hamdani and Døving, 2007; Sato et al., 2005; Thommesen, 1983).

Amphibians are the first tetrapods, that possessed an anatomically distinct vomeronasal system (Eisthen, 1997). In Xenopus laevis, microvillous VRNs project their axons towards an anatomically distinct AOB (Hansen et al., 1998; Nezlin and Schild, 2000). VRNs of the AOB express later diverging V2Rs (Hagino-Yamagishi et al., 2004; Syed et al., 2013) and lack any V1Rs (Date-Ito et al., 2008). In contrast to fish, the sensory surface of adult Xenopus MOS is anatomically divided into the principle and middle cavity. Those cavities emerge during metamorphosis by restructuring the larval principle cavity (the larval water-nose) into the adult air nose and the formation of a novel water-nose, the middle cavity (Altner, 1962; Dittrich et al., 2016; Hansen et al., 1998). The receptor expression and ORN types of the respective cavities are adapted to the detection of airborne or waterborne odorants (Altner, 1962; Hansen et al., 1998; Syed et al., 2017). However, there is no strict anatomical segregation between OR-type and VR-type odorant receptors, as observed in rodents and described earlier in this thesis: cAMP-dependent, microvillous and cAMP-independent, ciliated ORNs co-exist in the larval epithelium. A cAMP-independent, amino acid-sensitive ORN population, is located basally and shows a biased distribution towards the lateral part of the half-moon shaped olfactory mucosa of larval Xenopus (Gliem et al., 2013; Syed et al., 2013). The neuronal population is also present in the newly formed middle cavity of post-metamorphic Xenopus laevis (Syed et al., 2017). The principal and middle cavity can also be seen as functional subsystems if one takes their specific subtypes of odorant receptors into account. The middle cavity mainly expresses 'fish-like' OR type I receptors (Freitag et al., 1995; Mezler et al., 1999) phylogenetically ancestral subfamilies of V2Rs (Shi and Zhang, 2007; Syed et al., 2013, 2017) and V1Rs (Date-Ito et al., 2008). The principal cavity on the other hand expresses 'mammalian-like' OR type II (Freitag et al., 1995; Mezler et al., 1999) as well as V1Rs (Date-lto et al., 2008). 
Receptor families

'fish-like' ORs I

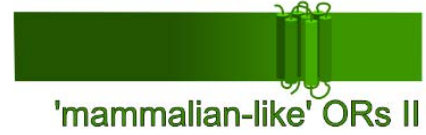

Zebrafish
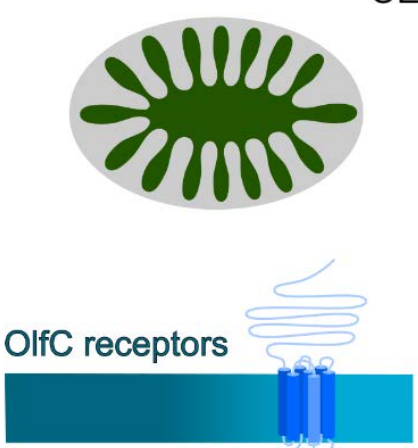

'mammalian-like' V2Rs

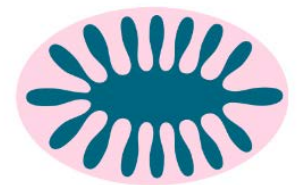

ORA receptors

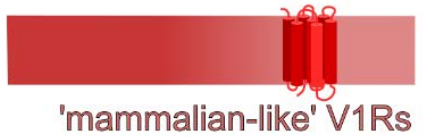

'mammalian-like' V1Rs
Xenopus

\section{Rodents}
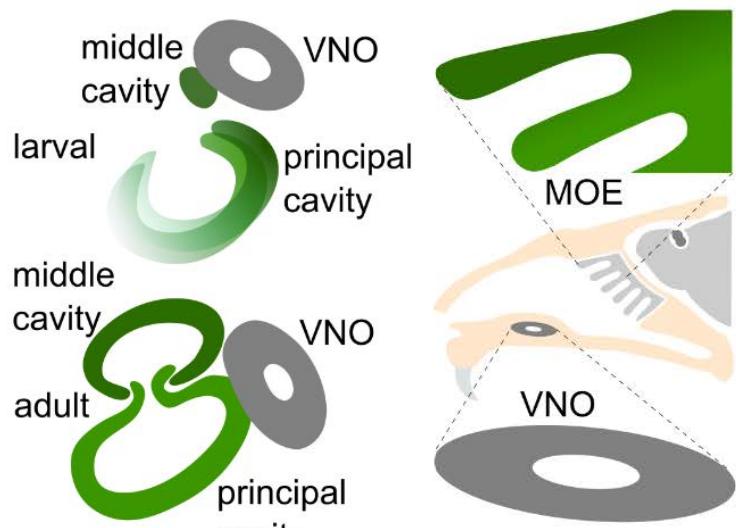

cavity
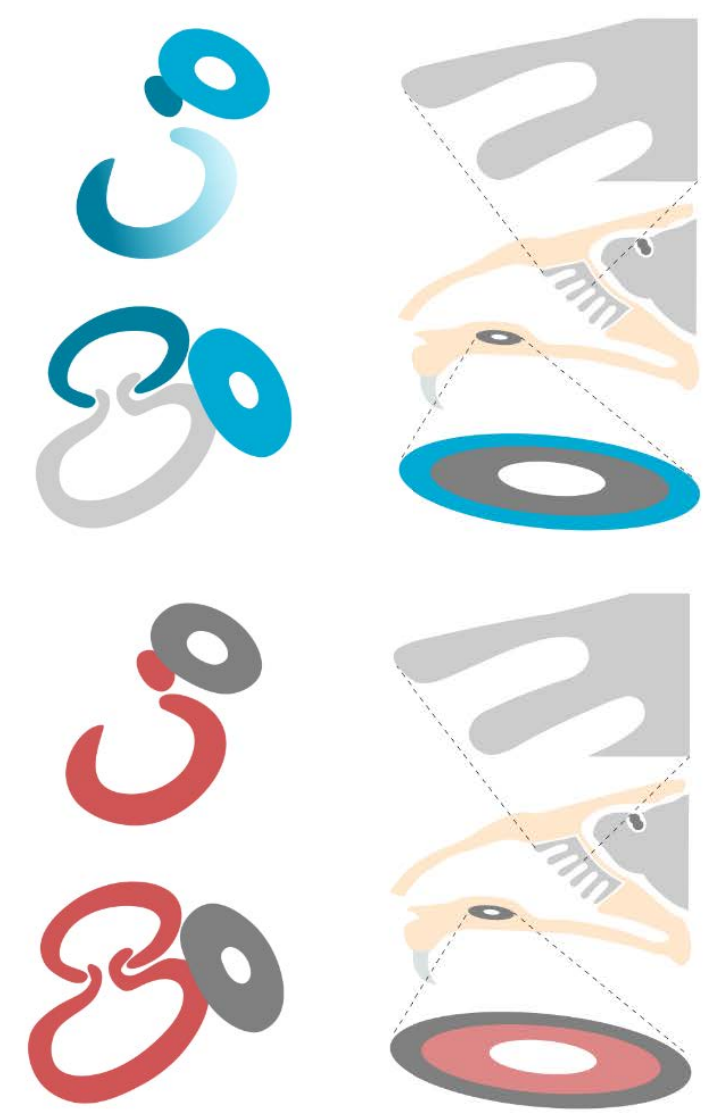

Schematic 4 Differential odorant receptor expression patterns in the olfactory subsystems of zebrafish, Xenopus and rodents

In the zebrafish the main odorant receptor classes (ORs, OlfCs and ORAs) are expressed on the single sensory surface (left row). The later diverging odorant receptor classes in rodents are expressed in different olfactory surfaces or distinct domains among these. ORs are found in the MOE of rodents with a gradient like dorsoventral distribution of more fish-like OR type I receptors to mammalian-like OR type II receptors (upper right). VRs are exclusively found in the VNO. V2Rs are expressed by a basal VRN population, whereas V1Rs are expressed by a more apical VRN population (lower left). In adult Xenopus OR type I receptors are expressed in the water-nose (middle cavity) whereas more mammalian-like OR type II receptors are expressed in the air nose (principal cavity). Amphibian V2Rs can be found in both the MOE (middle cavity, more ancestral V2Rs) and in the VNO (later diverging V2Rs). Xenopus V1Rs are exclusively expressed in the MOE (larval and adult middle and principal cavity). V2Rs and ORs are expressed in a spatially clustered but intermingled manner in the larval principal cavity. 


\subsection{ODORANT RECEPTOR EXPRESSION AND ORN AXONAL WIRING LOGIC}

\section{Axonal wiring logic and olfactory glomeruli}

\section{Axonal wiring logic in the rodent main olfactory system}

In the rodent MOS, OR-type ORNs express a single OR in a monoallelic fashion from a repertoire of around 1000 OR genes (Buck and Axel, 1991; Chess et al., 1994; Serizawa et al., 2000). The one-receptor-one-neuron hypothesis is supported by several studies, but should not be taken as absolute and cannot simply be extrapolated to other receptor families (Mombaerts, 2004b). ORNs expressing one individual OR project their non-bifurcating axons to two spatially defined regions, one in the medial and one in the lateral hemisphere of the MOB (Klenoff and Greer, 1998; Mombaerts, 1996; Ressler et al., 1994; Vassar et al., 1994). The ovoid-shaped neuropil structures formed by the coalescing ORN axons were named olfactory glomeruli by Rámon y Cajal (Figueres-Oñate et al., 2014). A population of ORNs that expresses the same receptor and projects to stereotypic glomeruli will, from now on, be referred to as ORN species. The relative positioning of individual glomeruli on the MOB surface is stereotypic between animals in rodents and dependent on its receptor expression and transduction machinery (Bozza et al., 2002; Feinstein and Mombaerts, 2004; Feinstein et al., 2004; Zapiec and Mombaerts, 2015). The positional precision of glomeruli is highly dependent on the individual receptor expressed by its ORN species (Zapiec and Mombaerts, 2015). Early studies using in situ hybridization proposed that rodent olfactory glomeruli are solely innervated by ORNs expressing one specific receptor (Ressler et al., 1994; Vassar et al., 1994). It is important to acknowledge that the limited subset of ORs investigated and the dynamic nature of the glomerular formation makes the innervation of one glomerulus by one ORN species working hypothesis rather than an absolute rule (Mombaerts, 2004b, 2006).

The MOB shows a zonal patterning of glomeruli along the dorsoventral axis that reflects the OR subfamily-dependent zonal ORN distribution observed in the MOE (Mori et al., 2000; Ressler et al., 1993; Vassar et al., 1993). The dorsal domains of the glomerular array receive mostly input from dorsal ORN populations expressing ORN populations expressing OR type or OR type II receptors, whereas the ventral glomerular domain is mostly targeted by ventral populations of ORNs expressing OR type II receptors (Miyamichi et al., 2005; Mori et al., 2000; Tsuboi et al., 2006). The ventrolateral organization of the glomerular array is conserved among the two mirror-symmetric glomerular maps of the OB (Nagao et al., 2000). The anatomical and functional organization of the glomerular map on a finer scale is far more complex than the coarse zonal division described. Key processes that influence the detailed distribution of glomeruli in the $\mathrm{OB}$ are a result of developmental processes (Mori and Sakano, 2011) and would exceed the scope of this thesis. 


\section{Axonal wiring logic in the rodent AOS}

VRNs reside in the VNO and project their axons to the AOB where they form synapses with their second-order neurons forming the glomerular array (Meredith, 1991). Odorant receptor expression of either the V1R or the V2R family instructs VRN axons to innervate either the rostral or the caudal part of the AOB in an exclusive manner (Belluscio et al., 1999; Rodriguez et al., 1999). In contrast to the MOS, VRNs that express one particular V2R or V1R mostly form several anatomically distinct glomeruli (Belluscio et al., 1999; Rodriguez et al., 1999). The odorant receptor choice for each VRN is an irreversible process as in OR-type ORNs and is believed to lead to the singular expression of one receptor from the respective gene cluster (Rodriguez, 2013). However, exceptions like the V2R families A,B and D that coexpress one receptor of the V2R-C family in a selective, non-random manner exist (Ishii and Mombaerts, 2011). The number of VRN axons making up an AOB glomerulus is more than tenfold smaller than in a MOB glomerulus (Meisami, 1989, 1991). Some of the glomeruli are less than $50 \mu \mathrm{m}$ in diameter and their anatomical borders are not as clearly delineated by glia cells as in the MOB (Meisami and Bhatnagar, 1998). In addition to unbranched VRN axons, some axons bifurcate or ramify before entering the $A O B$, even innervating several distinct glomeruli (Larriva-Sahd, 2008). A high variance in the positioning of glomeruli formed by individual VRN species is present among animals, but also between the same animals' paired AOBs (Belluscio et al., 1999). Positional variability of glomeruli exists in the MOB glomerular map, but in contrast to the AOB it is stereotypical and OR dependent (Zapiec and Mombaerts, 2015). Wagner and colleagues proposed a grid-like organization underlying the projection pattern of VRNs expressing receptors of the V1R family (Wagner et al., 2006). Instead of a fine spatial map, the AOB glomerular array is divided into subdomains targeted by specific subfamilies of V1Rs. Axons of VRNs expressing highly related receptors of a V1R subfamily appear to cluster together on a grid spanned between the dorsoventral and anterior-posterior axis of the AOB (Wagner et al., 2006). Instead of the molecular feature clusters observed in MOB chemotopic domains (Matsumoto et al., 2010; Uchida et al., 2000), the spatial organization of $\mathrm{AOB}$ clusters might serve to distinguish between behaviorally relevant groups of molecules, which are not necessarily homologous in their molecular structure (Hammen et al., 2014; Wagner et al., 2006).

\section{Axonal wiring logic and olfactory glomeruli in fish}

The majority of ciliated zebrafish ORNs that express canonical OR family genes investigated so far seem to follow the 'one neuron - one receptor' dogma (Barth et al., 1997). However, exceptions exist (Sato et al., 2007). The choice of receptors seems to be restricted to ORs that are part of the particular receptor subfamily and regulated as a hierarchical cluster during development (Sato et al., 2007). A different example of a 'one neuron-multiple receptor' configuration is the co-expression of broadly expressed OlfCc1 receptors with other OlfC clade members in microvillous neurons of zebrafish. (DeMaria et al., 2013). An early in vivo 
study by Dynes and Ngai revealed that independent of their target region in the zebrafish OB, individual developing ORNs' axons target single glomeruli in a deterministic manner (Dynes and Ngai, 1998). The unbranched axons were shown to undergo pruning of their axonal ramifications, but finally innervated single glomeruli (Dynes and Ngai, 1998). A more recent study by Shao and coworkers has analyzed axonal targeting of the OB in detail, taking into account the respective ORN family expression of the ORNs (Shao et al., 2017). During the early stages of differentiation, individual ORNs can co-express several OR genes. Nonetheless the majority of ORNs observed do finally project to defined proto-glomeruli in the OB (Shao et al., 2017). During development the zebrafish olfactory glomeruli undergo maturation from defined proto-glomeruli, until in mature zebrafish a mostly stereotypic glomerular map has formed (Braubach et al., 2012; Shao et al., 2017). Around 140 unambiguously identifiable glomeruli have been characterized by their location and immunohistochemical markers (Baier and Korsching, 1994; Braubach et al., 2012), pointing to a stereotypic axonal wiring logic as observed in rodents (Mombaerts et al., 1996). However, in the unparceled, lateral plexus in zebrafish, glomeruli were not easily identifiable (Braubach et al., 2012). This lateral part represents major parts of the TRPC2 positive neuronal ORN population described by Sato et al. 2005 and lacks Ga $_{\text {olf }}$ expression (Braubach et al., 2012; Sato et al., 2005). Similar to rodents, several single ORN species and their corresponding glomeruli have been characterized in fish. Virtually all of the ones investigated so far are associated with one to two individual stereotypic glomeruli although their odorant receptors and ligands are very different (Ahuja et al., 2013; Dieris et al., 2017; Wakisaka et al., 2017; Yabuki et al., 2016).

\section{Axonal wiring logic and olfactory glomeruli in amphibians}

In larval Xenopus laevis, the MOE and the VNO, convey their sensory information via the shared olfactory nerve towards their first central processing centers the anatomically distinct MOB and AOB (Nezlin and Schild, 2000). The MOB in larval Xenopus is located ventrally, whereas the dorsal portion is composed of an unstructured fiber meshwork in the pre- and pro-metamorphic stages (Nezlin and Schild, 2000). Anatomical and functional segregation exists on the level of ORN axonal projection areas in larval Xenopus OB (Gliem et al., 2013; Manzini et al., 2007a). The lateral axonal projection area, also called lateral cluster (LC), is proposed to be innervated by microvillous, cAMP-independent ORNs of the VR-type (Gliem et al., 2013; Manzini et al., 2007a; Nakamuta et al., 2011). In contrast, the medial cluster is composed of axons from cAMP-dependent and presumably mostly ciliated ORNs clustered in the more medial MOE (Gliem et al., 2013; Nakamuta et al., 2011). In addition to an intermediate cluster, a small cluster of specialized glomeruli exists in proximity to the extrabulbar fiber bundle (Manzini et al., 2007a). This cluster is among others involved in temperature and mechano-sensation and will not be discussed in this thesis (Kludt et al., 2015; Manzini et al., 2007a). The larval OB thus shows a lateral to medial zonal organization 
that is less strictly conserved in the olfactory mucosa (Gliem et al., 2013). After metamorphosis, ORNs of the middle cavity project their axons to the ventral OB. ORNs of the remodeled principal cavity innervate the newly formed dorsal OB (Dittrich et al., 2016; Hansen et al., 1998). The detailed anatomical organization of the glomerular clusters in the dorsal and ventral OB of adult Xenopus is unknown so far.

Functional experiments measuring odorant responses to amino acids in the larval MOE revealed a broad range of ORNs tuning profiles for the limited number of stimuli used. The sheer number of unique profiles could support the idea of odorant receptor co-expression in larval Xenopus (Manzini and Schild, 2004; Manzini et al., 2002). Apart from a hand full of studies that deduced receptor co-expression from overlapping expression zones of receptor families (f.ex. V2R-c and V2R; (Syed et al., 2013) barely anything is known about odor receptor choice in Xenopus laevis. There are no transgenic animal models that could help unravel the axonal wiring logic of Xenopus ORN so far. Two of the studies that have given a hint about the glomerular wiring logic in amphibians are single-cell tracing studies performed by Nezlin and Schild (2005) and Hassenklöver and Manzini (2013) in Xenopus. Those studies revealed a remarkable difference in the axonal wiring logic compared to the vertebrates observed to date: ORNs of both, the MOS and AOS, bifurcate in the OB and can innervate either one or multiple (mostly two) glomeruli (Hassenklöver and Manzini, 2013; Nezlin and Schild, 2005). This multi-glomerular wiring of ORNs is present in larval and adult animals (Hassenklöver and Manzini, 2013) and also among other amphibians (Weiss et al., 2019). A similar wiring scheme is not (yet) observed in any other terrestrial or aquatic vertebrate MOS to date (Dynes and Ngai, 1998; Larriva-Sahd, 2008; Mombaerts et al., 1996). Even more puzzling, the bifurcation and multi-glomerular innervation seem to exist in both the medial and lateral olfactory stream (Hassenklöver and Manzini, 2013; our unpublished data). 


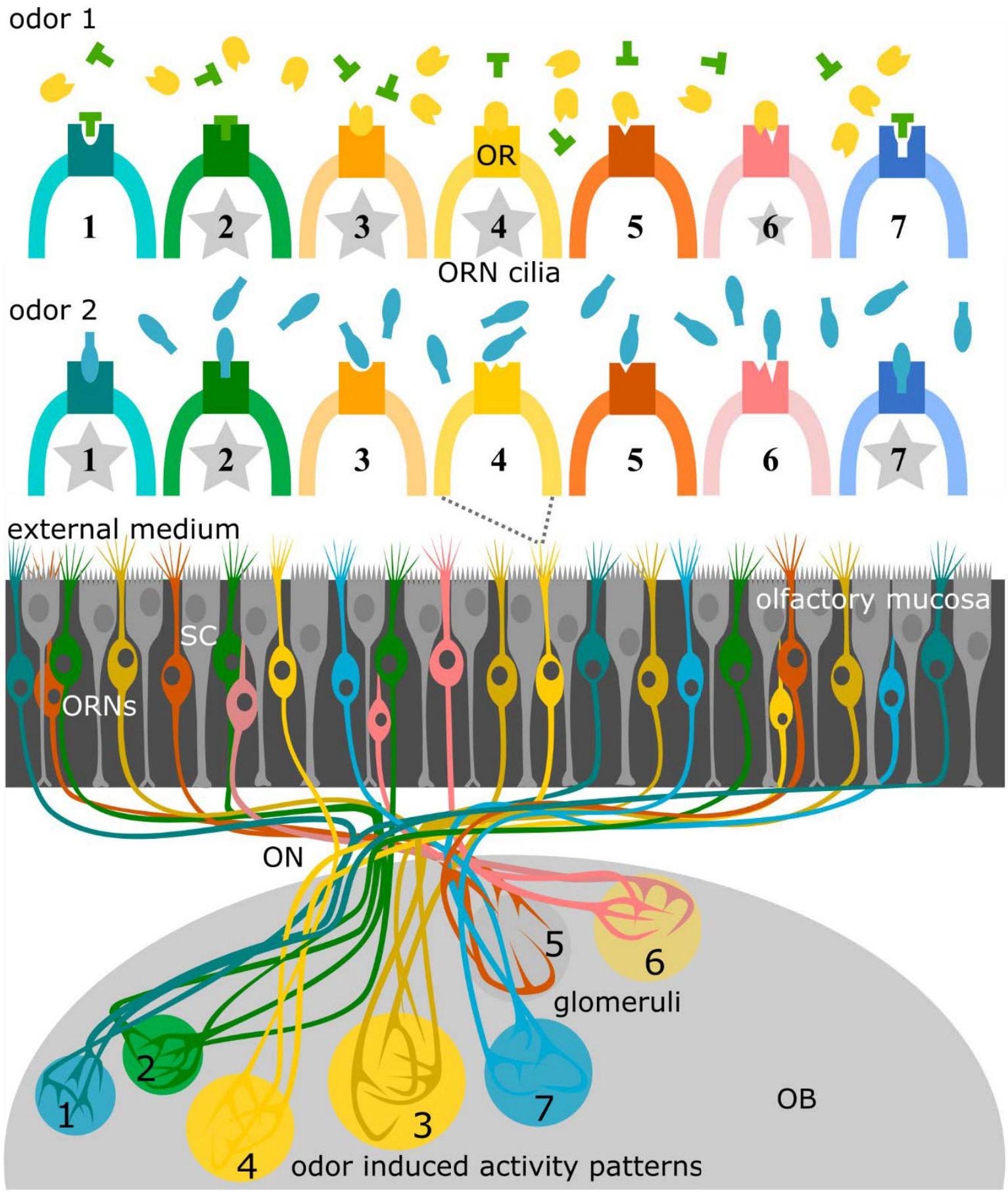

Schematic 5 Axonal wiring in the rodent MOS and glomerular odor representation

Combinatorial receptor coding (See Schematic 1) leads to stimulus-induced neuronal activity in subsets of ORN species (ORNs expressing one OR: individual colors). The axons of each ORN species converge onto olfactory glomeruli in the OB (numbered circles). The activity patterns of the different ORN species are translated into glomerular activity patterns (odor 1: glomeruli 2,3,4 and 6; odor 2: glomeruli 1, 2 and 7). Different strengths of activation (glomerulus 6) as well as partial overlap of glomerular representations (glomerulus 2) are key features of the odor representation in the rodent MOB. (SC: Supporting cells). 


\subsection{GLOMERULAR ODOR REPRESENTATION AND CHEMOTOPY}

\section{Glomerular odor maps}

The discoveries of the odorant receptor-dependent axonal wiring logic (Mombaerts, 1996) and that each olfactory glomerulus in the MOB receives the information of one OR (Ressler et al., 1994; Vassar et al., 1994) were essential to interpret the activity patterns of MOB olfactory glomeruli. The other fundamental mechanism that led to a better understanding of glomerular activity was the combinatorial receptor coding of odors, described earlier (Buck and Axel, 1991; Malnic et al., 1999). The spatio-temporal activity patterns of olfactory glomeruli are the primary odor representation of odorant binding patterns detected on the ORN level (Korsching, 2001). Activation of glomerular ensembles by single odorant molecules was initially used to assign molecular features to individual glomeruli. A broad range of techniques was used to unravel the 'chemotopy' of the glomerular array including autoradiography (Lancet et al., 1982; Sharp et al., 1975; Willhite et al., 2006), fMRI (Poplawsky and Kim, 2014; Schafer et al., 2006; Xu et al., 2003), calcium and voltage imaging (Charpak, 2001; Friedrich and Korsching, 1997, 1998; Wachowiak and Cohen, 2001) as well as genetic strategies to label neuronal subgroups and their activity (Bozza et al., 2004; Mombaerts et al., 1996; Storace and Cohen, 2017; Wachowiak et al., 2013). It should be noted that these glomerular odor representations are highly dynamic on the temporal scale. Moreover, they are shaped by the contributing neuronal network on both pre- and postsynaptic parts (Lizbinski and Dacks, 2018).

\section{Odorant map in rodents}

It has emerged as a common concept in olfactory science that odor input information is mirrored in the OB as a spatio-temporal activity pattern of glomerular ensembles (Wachowiak and Shipley, 2006). Taking into consideration that each glomerulus is composed of the ORN axonal part, MTC dendrites and neurites of interneurons, the individual activity in those glomerular sub-compartments can vary (Storace and Cohen, 2017; Wachowiak et al., 2004). Individual rodent glomeruli receive input from 1000s of ORN (Mombaerts et al., 1996; Ressler et al., 1994). Even though ORNs are located dispersed in their respective zone in the sensory surface (Mori et al., 2000; Ressler et al., 1993), there is no evidence so far that individual axons that innervate a glomerulus carry different latency information (Wachowiak et al., 2004). The activity patterns observed in ORN axon terminals associated with one glomerulus are highly correlated and independent of odor identity or concentration (Wachowiak et al., 2004). Consequently, the presynaptic part of the glomerulus seems to represent a homogeneous, synchronized input of odor information (Wachowiak et al., 2004). Mechanisms like electrical coupling via gap junctions between nerve fibers (Schwartz-Levey et al., 1992; Zhang and Restrepo, 2002). Rapid depolarization through accumulation of $\mathrm{K}^{+}$ions in the extracellular matrix of glomeruli (Friedrich and Korsching, 1998; Jahr and Nicoll, 1981) are supposed to contribute to this synchronicity. The resulting depolarization of ORN axon terminals in a 
glomerulus cause calcium influx at defined 'hot spots' and trigger subsequent glutamate release into the synaptic cleft (Wachowiak et al., 2004). This current model highlights the role of glomeruli in amplifying and enhancing incoming odor information rather than to conserve individual information streams from the respective ORNs (Wachowiak and Cohen, 2001; Wachowiak et al., 2004).

\section{Chemotopy and molecular feature clusters of the rodent MOB}

The multidimensionality of odor space in combination with the combinatorial code underlying odor detection makes odor representations less topologically ordered and intuitive as representations in the visual or auditory system (Korsching, 2001; Malnic et al., 1999). Nonetheless, in the MOB of most mammalian species investigated so far, a coarse grouping of glomeruli activated by structurally similar odor molecule features occurs (Igarashi and Mori, 2004; Matsumoto et al., 2010; Uchida et al., 2000). Glomeruli can be categorized by their molecular receptive range (MRR), which is a measure for their odor selectivity and directly correlated to the associated odorant receptor. It can be seen analogous to the receptive fields of the visual system (Hubel and Wiesel, 1959; Kuffler, 1953). Glomeruli that exhibit similar MRRs respond to structurally similar molecular features of odorant molecules. Glomeruli of similar MRR spatially cluster on the glomerular array of the dorsal OB in rodents. These groups of glomeruli activated by similar molecular features were named molecular feature clusters (Igarashi and Mori, 2004; Matsumoto et al., 2010; Uchida et al., 2000). The coarse chemotopy of the molecular feature clusters can show overlap between individual domains. Predictions of molecular feature development along certain trajectories on the glomerular array are not as easily possible as in other sensory systems (Korsching, 2002; Mori et al., 2006). Nevertheless, the chemotopy of glomeruli and molecular feature clusters in rodents is stereotypic and consistent between animals (Uchida et al., 2000). In fact, the molecular feature clusters seem to not only serve to distinguish different odorants but also correlate with perceived odor qualities associated with their overarching molecular features (Mori et al., 2006).

AOS olfactory chemosensory map: receptor family and biological feature-based topology

The fundamentally different wiring logic underlying the AOS leads to an entirely different distribution of olfactory glomeruli (Belluscio et al., 1999; Rodriguez et al., 1999). Since VRNs that express one VR type receptor project into multiple glomeruli (VRN species), many of the small, less-parceled AOB glomeruli carry odor information of the same receptor (Belluscio et al., 1999; Larriva-Sahd, 2008; Rodriguez et al., 1999). The only extensive study so far mapping glomerular activity to complex odors and single molecular compounds known to activate VRNs was performed by Hammen and colleagues in 2014. Their calcium imaging experiments revealed several important differences between the glomerular map organization of $\mathrm{AOB}$ and $\mathrm{MOB}$ : First, glomeruli exhibiting similar odor tuning were not 
necessary found spatially clustered but occurred rather dispersed in both the rostral and caudal AOB (Hammen et al., 2014). Second, the proximity of glomeruli correlated rather with the amino-acid sequence similarity of their VRs. The juxtaposition of glomeruli and their MRR were not correlated (Hammen et al., 2014; Wagner et al., 2006). Intriguingly, glomeruli of the $A O B$ seem to be clustered rather by their biological relevance and features associated with a certain biological phenotype, like gender, age, or sexual maturity (Hammen et al., 2014). The rodent AOS is highly and broadly responsive to urine (Ben-shaul et al., 2010; Chamero et al., 2012; Holy, 2000). Urine represents a complex blend of structurally heterogeneous molecules. However, urine samples taken from different biological phenotypes activate spatially clustered glomeruli (Hammen et al., 2014). Sulfated steroids represent a structurally related group of odorants that can activate large groups of V1Rs and V2Rs (Hammen et al., 2014; Meeks et al., 2010; Nodari et al., 2008; Turaga and Holy, 2012). Single sulfated steroids that display high molecular similarity were not found to activate adjacent glomeruli (Hammen et al., 2014). The concentration dependence of glomerular representation, as seen in the $M O B$, exists as well in the AOB. Some sulfated steroids elicited significant responses already at concentrations of $1 \mu \mathrm{M}$. The number of recruited glomeruli with $100 \mu \mathrm{M}$ odor concentration increased dramatically, but still in a stimulus-specific manner (Hammen et al., 2014). Overall the functional glomerular map of the rodent $A O B$ is fundamentally differently organized than the MOB.
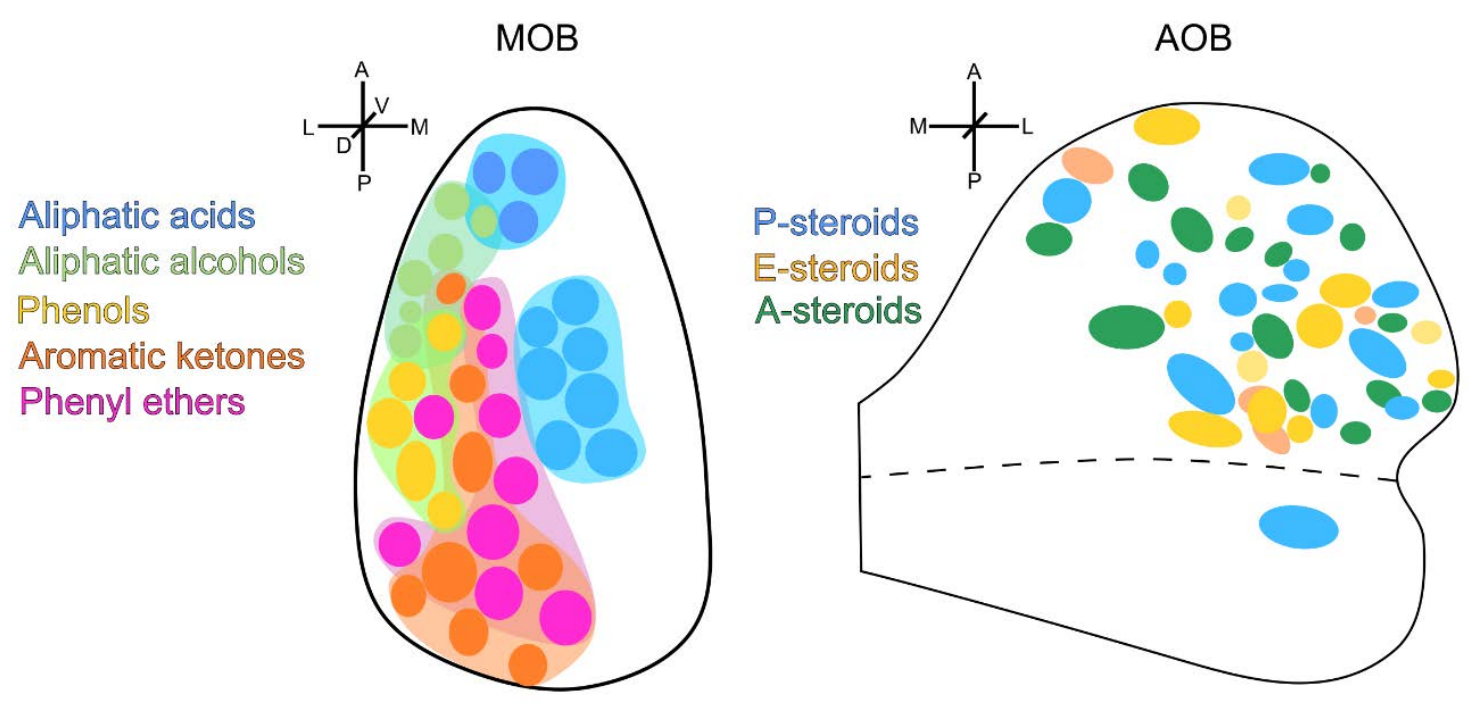

Schematic 6 Differences in odor representation between the rodent MOS and AOS

In the rodent $\mathrm{MOB}$, glomeruli spatially cluster according to the structural features of odorants they detect (left panel). These territories (molecular feature clusters: colored areas) can be overlapping, but are organized in a coarsely chemotopic manner (odorant groups and respective glomeruli are indicated in the individual colors). The rodent $A O B$ is organized in a non-chemotopic manner (right panel). Glomeruli activated by structurally similar molecules (steroid subgroups: different colors) appear dispersed among the entire anterior AOB and glomeruli of dissimilar tuning can be found adjacent to each other. 


\section{Odor maps in fish}

Some of the pioneering studies measuring presynaptic glomerular odor responses with calcium and voltage-sensitive dyes were performed in zebrafish (Friedrich and Korsching, 1997, 1998). Important findings of those studies were, that some territories on the glomerular array showed chemotopic and combinatorial odor representations, whereas other parts displayed rather focal, non-combinatorial glomerular activity (Friedrich and Korsching, 1998). The focal, non-combinatorial activity of individual glomeruli to water-borne odorants like ATP, prostaglandin F2alpha (FGF), and cadaverine as well as their underlying odorant receptors have been described in zebrafish. Those glomeruli act as single-channel subsystems in zebrafish with immediate behavioral consequences (Ahuja et al., 2013; Dieris et al., 2017; Wakisaka et al., 2017; Yabuki et al., 2016). Other odorant molecules activate glomerular subsets in a combinatorial, concentration-dependent manner (Friedrich and Korsching, 1997, 1998)similar to the AOB of rodents (Fried et al., 2002; Meister and Bonhoeffer, 2001). The lateral part of the zebrafish OB is responsive to amino acids (Friedrich and Korsching, 1997, 1998), an attractive, food-related stimulus (Koide et al., 2009), whereas the medial part responded to structurally different bile acids (Friedrich and Korsching, 1998). A coarse chemotopy was present in the amino-acid sensitive lateral glomerular cluster (Friedrich and Korsching, 1997). The glomerular subsets activated by the different groups of amino acids occupied distinct, but partially overlapping territories, as observed in the molecular feature clusters in rodents (Igarashi and Mori, 2004; Matsumoto et al., 2010; Uchida et al., 2000). It should be noted though, that while most molecular feature clusters described in rodents are based on glomeruli formed by OR type I or type II ORNs, the glomeruli of the lateral plexus in zebrafish might be associated with different odorant receptors and signal transduction cascades (Sato et al., 2005).

\section{Odor maps in amphibians}

While the coarse tuning of the glomerular clusters to certain odor groups in Xenopus laevis have been investigated (Gliem et al., 2013) the only detailed studies addressing single glomeruli and their response profiles were performed in slice preparations of the LC (Manzini et al., 2007b) and in two glomeruli associated with the small glomerular cluster (Brinkmann and Schild, 2016; Kludt et al., 2015). The latter represent non-canonical solitary glomeruli, that process additional modalities like mechano-sensitivity and temperature (Brinkmann and Schild, 2016; Kludt et al., 2015). Amino acid-sensitive glomeruli and their odor tuning were described in the LC of larval Xenopus in vitro by Manzini and colleagues. The odor map, as well as the positioning of individual glomeruli, were not addressed in this study (Manzini et al., 2007b). The amino-acid sensitive lateral glomerular cluster of Xenopus is often compared to the lateral plexus in zebrafish (Friedrich and Korsching, 1997; Manzini et al., 2007b). However, there are no detailed studies that confirm a similar glomerular array organization. 


\section{From glomerular maps to postsynaptic network activity}

Sensory input that can be detected by an animal's odorant receptor repertoire is gathered in the peripheral olfactory system and translated into a spatio-temporal activity pattern mirrored on the glomerular array (Korsching, 2001; Wachowiak and Shipley, 2006). The output information of the OB is encoded in the spatio-temporal activity of MTCs, to be more precise, in the activity patterns conveyed by their axons to higher olfactory brain centers (Allison, 1953; Igarashi et al., 2012). However, MTCs are not just passive connectors, but act as an intermediate level of odor representation and odor information processing (Cavarretta et al., 2018). To understand odor representations on the pre- and the postsynaptic levels of the glomerular array, it is crucial to comprehend the morphology, connectivity and intrinsic properties of MTCs.

\subsection{MTCS - THE VERTEBRATE OLFACTORY PROJECTION NEURONS Basic blueprint of MTCs}

MTCs are the major neuronal mediators between the peripheral olfactory system and the higher brain centers involved in odor processing and perception. They receive sensory input from hundreds of ORNs (Meisami, 1989) and convey the information via their axons to higher brain centers (Allison, 1953; Igarashi et al., 2012; Pinching and Powell, 1971a). The morphological blueprint of these cells is a good reflection of their three major functions. Thick apical primary dendrites emerge from the soma, branch, and form extensive dendritic arborizations called tufts. Tufts are dendritic specializations that form synapses with hundreds of ORNs and interneurons associated with the glomeruli (Pinching and Powell, 1971a). Depending on the species or the specific requirements for odor processing, MTCs can bear several secondary dendrites of various lengths and destinations (Dryer and Graziadei, 1994). These dendrites are potential synaptic sites with other neuronal cell types involved in odor processing and can shape the actual odor output of the MTC. The MTCs' axons and axon collaterals convey the integrated odor information to several higher brain centers (Igarashi et al., 2012). Despite this conserved general outline of MTC morphology, the composition of the individual features and dendritic configurations show high variability in vertebrates (Dryer and Graziadei, 1994). This variability might be a result of evolutionary adaptations to different lifestyles and the associated challenges of odor detection and processing. However, this does not imply that the variability of MTC morphology cannot vary even among species of similar lifestyle or environment (Dryer and Graziadei, 1994).

\section{MTCs in the rodent MOS}

The name mitral cell (MC) originates from the mitre like shape of these neurons' somata. The other major group of output neurons of the $\mathrm{OB}$, the tufted cells, were named after their 
dendritic arborizations reminding of brushy tufts. The architecture and cellular morphologies of the OB were first described by Ramon y Cajal and contemporaries (reviewed in (FigueresOñate et al., 2014). Extensive follow-up Golgi impregnation studies performed in the 1970s led to a morphological categorization of the main types of projection neurons in the mammalian OB, and to the definitions of histological layers (Pinching and Powell, 1971a; Price and Powell, 1970). In the concentrically layered MOB of rodents the distinguishable histological layers, starting from the OB surface towards the ventricle are: the nerve layer $(N L)$, the glomerular layer $(G L)$, the external plexiform layer (EPL), the mitral cell layer (MCL), the internal plexiform layer (IPL) and the granule cell layer (GCL; Pinching and Powell, 1971a, 1971b; Price and Powell, 1970). MC somata align in the well-defined MCL, while tufted cells are more dispersed in the neuropil-rich EPL between the MCL and the GL (Macrides and Schneider, 1982). The EPL is mainly composed of dendritic networks of MCs, tufted cells and granule cells (GCs; Pinching and Powell, 1971a, 1971b; Price and Powell, 1970) and several types of interneurons (Schneider and Macrides, 1978). Moreover, the group of tufted cells was additionally subdivided into internal tufted cells residing in the deeper layers of the EPL, middle tufted cells in the intermediate EPL and a third type located in close proximity to the transition zone between the GL and superficial EPL, the external tufted cells (ETCS; Macrides and Schneider, 1982). ETCs' primary dendrites are usually associated with one individual glomerulus, and only rarely with two (Hayar et al., 2004a, 2004b) They have the biggest somatic diameter of the juxtaglomerular cells (JGCs) and extend extensive ramifications into the glomerular volume (Nagayama et al., 2014; Pinching and Powell, 1971b). Middle tufted cells innervate one glomerulus with their apical tufted dendrite and will, from now on, be referred to as TCs. They extend secondary dendrites into the EPL (Nagayama et al., 2014). Axons of TCs project to higher brain centers like the anterior olfactory nucleus and the olfactory tubercle (Igarashi et al., 2012). MCs are the largest OB output neurons ( $20 \mu \mathrm{m}$ diameter) and were further subdivided into type I or type II mitral cells according to their secondary dendritic projection (Nagayama et al., 2014). Type I cells dendrites project to the deeper EPL while type II mitral cells dendrites project to the intermediate EPL (Mori et al., 1983; Orona et al., 1984). Their long secondary dendrites span entire parts of the EPL. Axon collaterals of MCs project to almost the entire olfactory cortex, but they target different regions than TCs (Igarashi et al., 2012). Unlike the other tufted cell types, MCs have not been observed to form connections with the mirror-symmetric contralateral side of the OB (Igarashi et al., 2012).

\section{MTCs of the rodent AOS}

AOB neurons were named analogous to their counterparts in the MOB (Larriva-Sahd, 2008). Despite the lack of a mitre-like shape, the principal output neurons of the rodent $A O B$ are often referred to as accessory mitral cells (AMCs; Mohrhardt et al., 2018). The AOB is histologically divided into the vomeronasal nerve layer, glomerular layer, external cellular layer and internal cellular layer. The dendritic variability among the AMCs is higher than in 
the MOB (Larriva-Sahd, 2008). Detailed morphological studies have divided the AMCs into the large principal cells, round principle cells and tufted cells from here on referred to as ATCs (Larriva-Sahd, 2008). The size and shape of large principal cells' somata vary but are correlated with the number of dendrites projecting to the GL. Those glomerular dendrites are thick, and each large principal cell bears multiple (3-9) of them (Larriva-Sahd, 2008; Takami and Graziadei, 1991). The additional thinner dendrites were classified according to where they project to in the AOB. Local dendrites project to the homonymous and commissural dendrites to the opposite half of the AOB (Larriva-Sahd, 2008). The majority of large principal cells' axons emerge from the soma or dendritic trunks and bypass the associated as well as the opposite $A O B$ half several times before leaving the $A O B$ towards higher brain centers (Larriva-Sahd, 2008). On their way, they form decussating collaterals innervating both to the homonymous and the opposite AOB half (Larriva-Sahd, 2008). While corresponding to the TCs in the MOB, the tufted cells of the AOB (ATCs) should be considered local interneurons (Larriva-Sahd, 2008), since their axons do not project to higher brain centers as in the MOS (Igarashi et al., 2012). In contrast to the large principal cells, they extend a single dendrite to one individual glomerulus from their smaller ellipsoid soma (Larriva-Sahd, 2008).

\section{MTCs in fish}

The MTCs of fish represent the most ancient group among the ubiquitous vertebrate projection neurons (Dryer and Graziadei, 1994). The histological layering of the OB seen in mammalian systems does not exist to the same extent in fish. A coarse laminar organization into four layers was proposed (Byrd and Brunjes, 1995; Kermen et al., 2013). Due to the diffuse borders between the layers, fish MTCs are mostly characterized by their morphology (Kosaka and Hama, 1982; Oka, 1983). In fish investigated so far, MTC morphologies appear very heterogeneous (Dryer and Graziadei, 1994). A shared feature of fish MTCs is the absence of basal secondary dendrites. In contrast to mammals, MTCs with multiple primary dendrites exist that can innervate one up to several glomeruli (Dryer and Graziadei, 1994; Oka, 1983). Due to their variable morphologies and lack of comparable layering, it is not clear whether functional subtypes like MCs and TCs exist in the fish olfactory system. Zebrafish MTCs share those features mentioned above, but in harsh contrast to other investigated teleost species multi-dendritic types predominantly innervate a single glomerulus (Dryer and Graziadei, 1994; Fuller et al., 2006; Wanner et al., 2016). The soma varied in shape and size and was indicative of the existence of multiple dendrites (Fuller et al., 2006). The individual MTC's position in the OB along the medio-lateral axis was a solid predictor whether its axon would converge into the lateral or medial olfactory tract (Fuller et al., 2006; Sato et al., 2005). 


\section{MTCs in amphibians}

The most characteristic morphological difference between Amphibian MTCs and the ones of fish is the existence of basal dendrites (Dryer and Graziadei, 1994; Scalia et al., 1991). Moreover, due to the longer primary dendrites compared to fish, an EPL can be histologically distinguished (Scalia et al., 1991). Primary dendrites can emerge from basal dendrites. The multiple primary dendrites were frequently observed to innervate multiple anatomically distinct glomeruli (Jiang and Holley, 1992). The multi-dendritic and multi-glomerular configuration of MTCs has been confirmed in the OB of larval Xenopus laevis (Nezlin and Schild, 2005). Whether these MTC morphologies are still present in the adult OB after metamorphosis or whether certain MTC subtypes exist in Xenopus laevis, is not known yet.

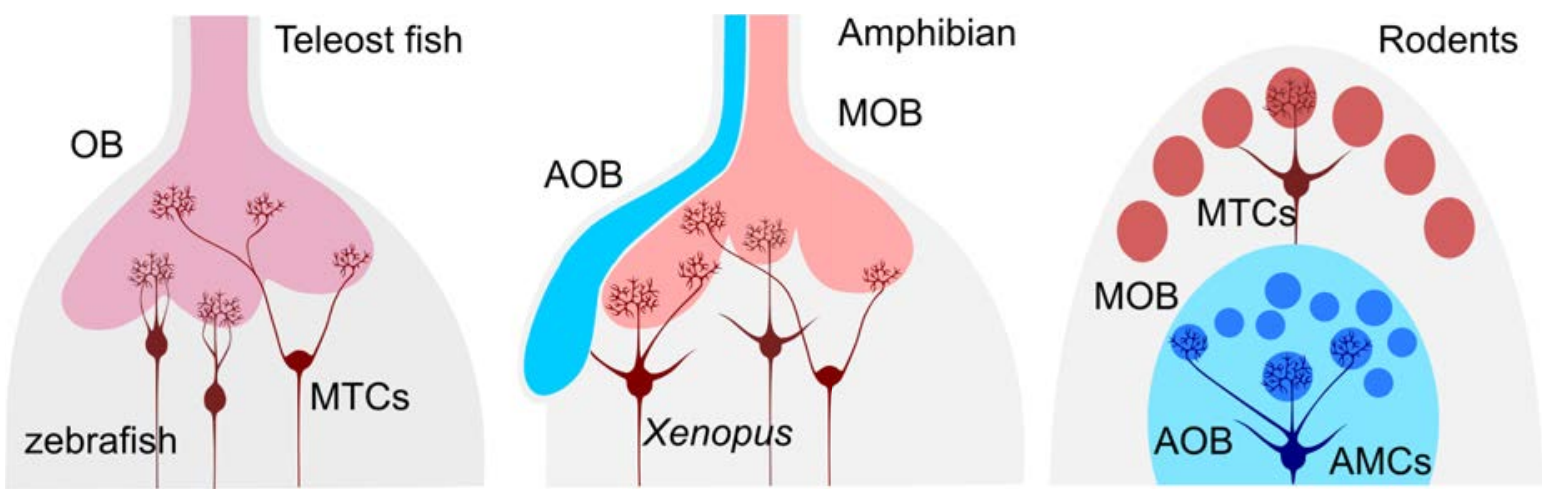

Schematic 7 Morphological differences between MTCs of fish, amphibians and rodents

Teleost fish mostly possess MTCs with multiple tufted dendrites and no basal dendrites. While in zebrafish the uni-glomerular wiring of MTCs dominates, in other teleost fish MTCs receiving multi-glomerular input occur frequently. Amphibians like Xenopus possess multi-dendritic MTCs with basal dendrites. They can either innervate individual or numerous glomeruli with their primary dendrites. In rodents, the MTCs (MCs and TCs) possess secondary dendrites and innervate one glomerulus with their apical primary dendrite. AMCs, the projection neurons of the AOS, usually innervate numerous glomeruli with their tufted dendrites.

\subsection{MTCS AND GLOMERULAR MODULES}

The basic cellular components and fundamental wiring strategy of the olfactory system circuitry is very conserved among vertebrate species (Hildebrand and Shepherd, 1997). One species of ORNs, is defined by its odorant receptor expression profile and projects its axons to the OB where it synapses with a certain subset of TCs and MCs (Mombaerts, 1996; Ressler et al., 1994; Vassar et al., 1994). MCs integrating the input from the same odorant receptor species are often referred to as sister MCs (Dhawale et al., 2010). Given the fundamental importance of the odorant receptor identity in axonal coalescence to glomeruli (Mombaerts et al., 1996; Ressler et al., 1994; Vassar et al., 1994), the ensemble of neurons connected to a single glomerulus is also often termed glomerular module analogous to cortical network modules (Chen and Shepherd, 2005). Considering the high prevalence of multi-dendritic MTCs among vertebrates (Dryer and Graziadei, 1994; Nezlin and Schild, 2005) and the existence of ORN axonal bifurcations innervating several glomeruli (Hassenklöver and Manzini, 2013; Larriva-Sahd, 2008; Weiss et al., 2019) the definition of glomerular modules needs to be more adaptive. To account for the possibility of several wiring logics (co-)existing in different animal classes, I will from now on use the term glomerular module 
for all cells receiving direct excitatory input (mono-synaptically or via gap junctions) from the axon terminals of one specific ORN species (concept modified (Chen and Shepherd, 2005).

\subsection{UTPUT NEURONS AS FUNCTIONALLY COUPLED EXCITATORY MODULES}

Gap junctions or electrical synapses are an efficient way to electrically couple individual neurons to form a functional entity. Gap junctions have shown to be critical at several levels of OB processing, especially between neurons associated with individual glomeruli (Chen et al., 2009; Hayar et al., 2004a; Zhang and Restrepo, 2002). ETCs and MCs were shown to form gap junctions with their sister cells associated to the same glomerulus. The synchronized release of excitatory neurotransmitter from the ORN part of the glomerulus leads to a synchronized activation of glomerulus associated ETCs, boosting of their intrinsically rhythmic activity (Hayar et al., 2004a, 2004b, 2005; Wachowiak et al., 2004). Recently a paradigm shift in glomerular signal transmission has happened: ETCs mainly drive MC excitation via feed-forward excitation and/or gap junctions with MC dendrites (Gire et al., 2012; Najac et al., 2011; De Saint Jan et al., 2009) In addition to electrical coupling, glutamate release by the $M C$ dendrite itself can lead to self-excitation via glutamate auto-receptors (Salin et al., 2000). 'Glutamate spillover' in the synaptic cleft can, in addition, excite adjacent MCs through AMPA and NMDA receptors (Isaacson, 1999; Schoppa and Westbrook, 2001). Experiments performed on pairs of TCs cells revealed that also sister TCs seem to be electrically coupled (Ma and Lowe, 2010). The two excitatory axes (ORN-ETC-MC and ORNTC) are capable of conveying highly synchronous temporal activity patterns within milliseconds as well as at the level of hundreds of milliseconds (Christie et al., 2005; Schoppa and Westbrook, 2001). There is barely anything known about sister MTCs in fish. However, in Xenopus laevis, electrical coupling, and highly correlated activity between sister MTCs have been reported (Chen et al., 2009). Chen and colleagues could show that uni-glomerular sister MTCs exist in larval Xenopus laevis. However, their experiments could not reveal whether this was also true for the abundant multi-glomerular MTC type (Chen et al., 2009; Nezlin and Schild, 2005). Synchronization through excitatory mechanisms are just a part of the complex multiplexed neuronal code used by MTCs. In fact, their spatio-temporal activity patterns cannot be understood without neuromodulation exerted by interneurons at several levels and timescales.

\subsection{NEUROMODULATION OF INDIVIDUAL GLOMERULAR MODULES BY INTERNEURONS IN THE GL}

Neuromodulation is the alteration of biophysical properties and synaptic transmission of neurons by other neurons in neuronal networks (Katz and Calin-Jageman, 2009; Kupfermann, 1979; Lizbinski and Dacks, 2018). Neuromodulation can be divided into different categories. Intrinsic neuromodulation describes neuromodulation among connected neurons of a 
network module that processes shared information. Extrinsic neuromodulation is modulation of a neuronal network's activity by another neuronal network module that processes independent information (Katz and Calin-Jageman, 2009; Katz and Frost, 1996; Lizbinski and Dacks, 2018). MTCs and the invertebrate equivalents are the main targets of intra-bulbar neuronal modulation at several levels in the OB (Cavarretta et al., 2016; Lizbinski and Dacks, 2018). In this work, I will mainly address the intrinsic neuromodulation of MTCs at the glomerular level. A single glomerulus is composed of the ORNs axons (ON part) and the interdigitating non-ON part that made up by OB neurons' dendrites (Kosaka et al., 1998). Apart from the tufted dendrites of MTCs, most of the glomerular non-ON part consists of extensive arborizations from periglomerular cells (PGCs(Kosaka et al., 1998; Pinching and Powell, 1971b) PGCs' dendrites extend throughout the entire glomerulus and can receive direct monosynaptic input from ORNs (Kosaka and Kosaka, 2016; Kosaka et al., 1998; Pinching and Powell, 1971a, 1971b). The most common form of intra-glomerular neuromodulation of MTCs is feed-forward inhibition. This inhibition can be exerted via different microcircuits: The ORN-PG-MTC, ORN-ETC-PG-MTC, or MTC-PG-MTC pathway (Wachowiak and Shipley, 2006). Depending on which pathway is used, immediate or longerlasting inhibitory events can be triggered to sculpt the temporal activity pattern conveyed to the output neurons soma (Wachowiak and Shipley, 2006). The main neurotransmitter involved in intra-glomerular inhibition by PGs is gamma aminobutyric acid (GABA). However, the entity of JGCs represents a heterogeneous group of interneurons (Kosaka and Kosaka, 2005, 2016; Kosaka et al., 1998; Nagayama et al., 2014). They can also exert modulatory effects by other neuropeptides like CCK and somatostatin or dopamine (Gutièrrez-Mecinas et al., 2005; Morona and González, 2013). 


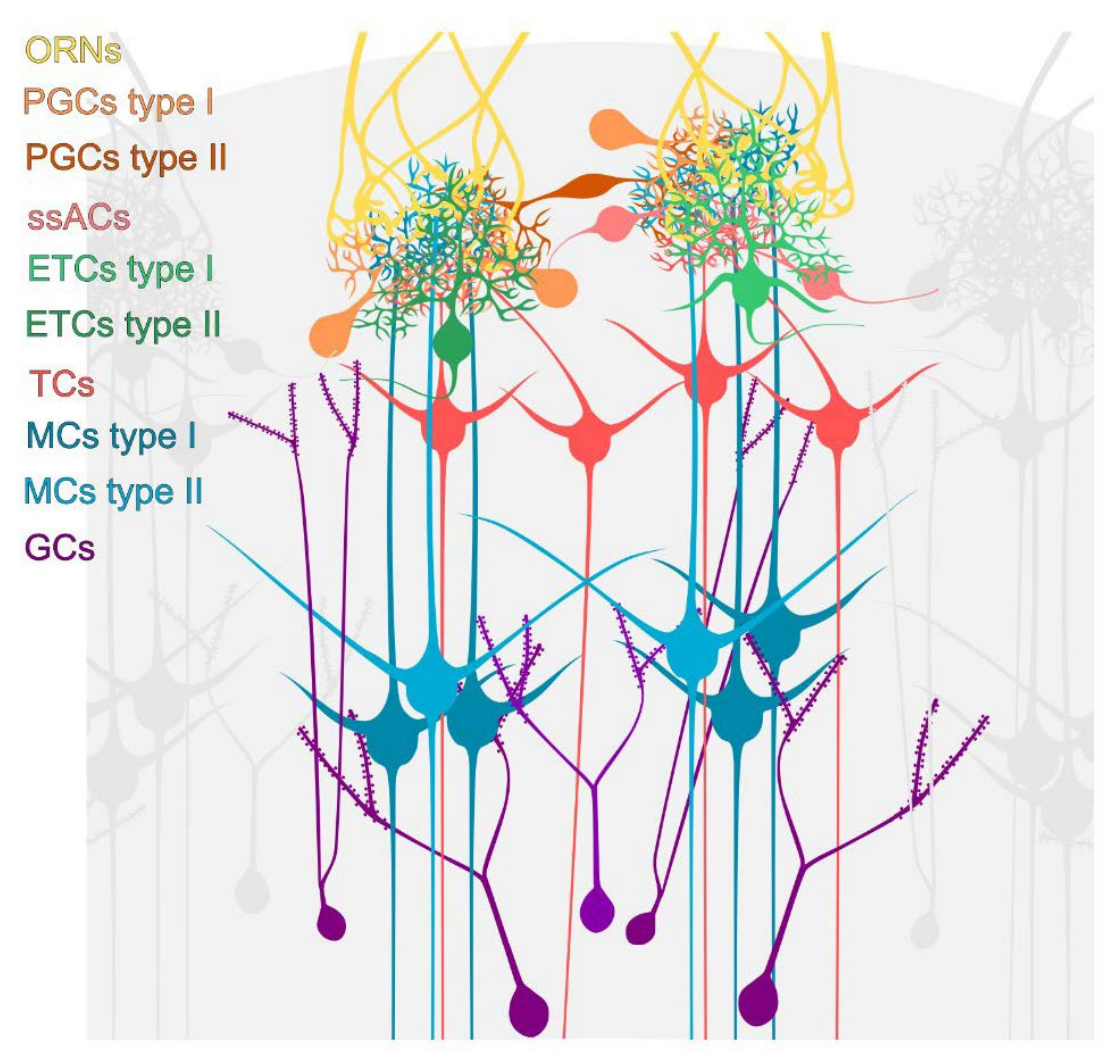

Schematic 8 Main cell types of glomerular modules in the rodent MOB

Schematic representation of two glomerular modules in the rodent MOB. The individual cell-types of the modules are highlighted in different colors. ORN input (yellow) is relayed onto primary tufted dendrites of TCs (red), MCs (type I, dark blue; type II, light blue) and a set of PGCs (light orange) and ETCs (green). PGCs and ETCs can be associated with one glomerulus (orange) or rarely with two (dark orange). ETCs can possess secondary dendrites (dark green) and can be coupled to superficial short axonal cells (light red). GCs form dendro-dendritic synapses with MCs and TCs of the same (dark purple) or different glomerular modules (light purple).

\subsection{ODOR INFORMATION PROCESSING IN THE OB NEURONAL NETWORK}

\section{Neuronal code and multiplexing in sensory system}

The discovery of the action potential and neuronal spikes as a unit of information processing in the brain has revolutionized neuroscience and the endeavor of understanding neuronal coding strategies. A detailed review of neuronal coding would certainly exceed the frame of this dissertation. This paragraph's purpose is to briefly introduce relevant terms and main models of neuronal coding that are essential to my work in the olfactory system. (For a great review on the topic, see (Panzeri et al., 2010). The two basic dimensions of the neuronal code are space and time. Different populations of neurons carry the spatial dimension of the neuronal code. These populations can exist as either spatially distinct or intermingled neuronal network modules (Pouget et al., 2000). The temporal dimension of neuronal coding is defined by the changes in activity patterns of neurons over time (Victor, 2000). Like yin and yang, none of the dimensions can explain the neuronal code on its own, but they are rather two parts of an entity (Panzeri et al., 2010). Both the population, as well as temporal coding mechanisms, play essential roles in the olfactory system. In this work, I focused more on the population coding carried by MTCs and their associated glomerular modules. 


\subsection{POPULATION CODING IN MTCS AND GLOMERULAR MODULES IN THE MOS}

In the rodent $\mathrm{MOB}$, the excitatory input to a single glomerulus is highly correlated in timing and represents a single ORN species' odor induced activity (Wachowiak et al., 2004). The OB network's capacity to extract and process primary sensory input is remarkable and to a big extent, achieved by different forms of neural coding of the neuronal networks involved.

\section{Parallel processing of odor modalities by MCs and TCs in the glomerular module}

There are two major consequences of combinatorial receptor coding (Malnic et al., 1999). First, odor representations of similar odorants or odor blends lead to broadly distributed, overlapping glomerular representations (Fried et al., 2002; Meister and Bonhoeffer, 2001). The second consequence is that the glomerular odor representation is concentrationdependent due to the activation of more glomeruli with increasing concentration (Fried et al., 2002; Storace and Cohen, 2017). The postsynaptic olfactory network has developed numerous computational mechanisms to extract essential information about odor identity and concentration. One of the mechanisms is the parallel processing of glomerular input by MCand TC-associated microcircuits (Cavarretta et al., 2018; Igarashi et al., 2012). TCs are now believed to carry mainly odor identity information on the short timescale of 100-150 ms. They provide stable odor representations over a broad range of different concentrations (Burton and Urban, 2014; Igarashi et al., 2012). On the circuit level, this higher sensitivity to glomerular input is also reflected by the TCs broader MRRs in comparison to MCs (Kikuta et al., 2013). MC and TC activity might also occur temporally segregated to a certain extent. TCs respond with significantly lower latencies to odorant stimulation then MCs (Igarashi et al., 2012). Behavioral experiments indicate that mice are capable of discriminating odorants on the timescale of $100 \mathrm{~ms}$ (Uchida and Mainen, 2003). MCs have higher thresholds for odor concentrations and respond with higher latencies to odorant stimulation (Igarashi et al., 2012; Kikuta et al., 2013; Nagayama et al., 2004). The different temporal activity patterns of MCs in combination with their narrower odor tuning thus provide a second layer of odor information (Igarashi et al., 2012; Kikuta et al., 2013; Nagayama et al., 2004). 


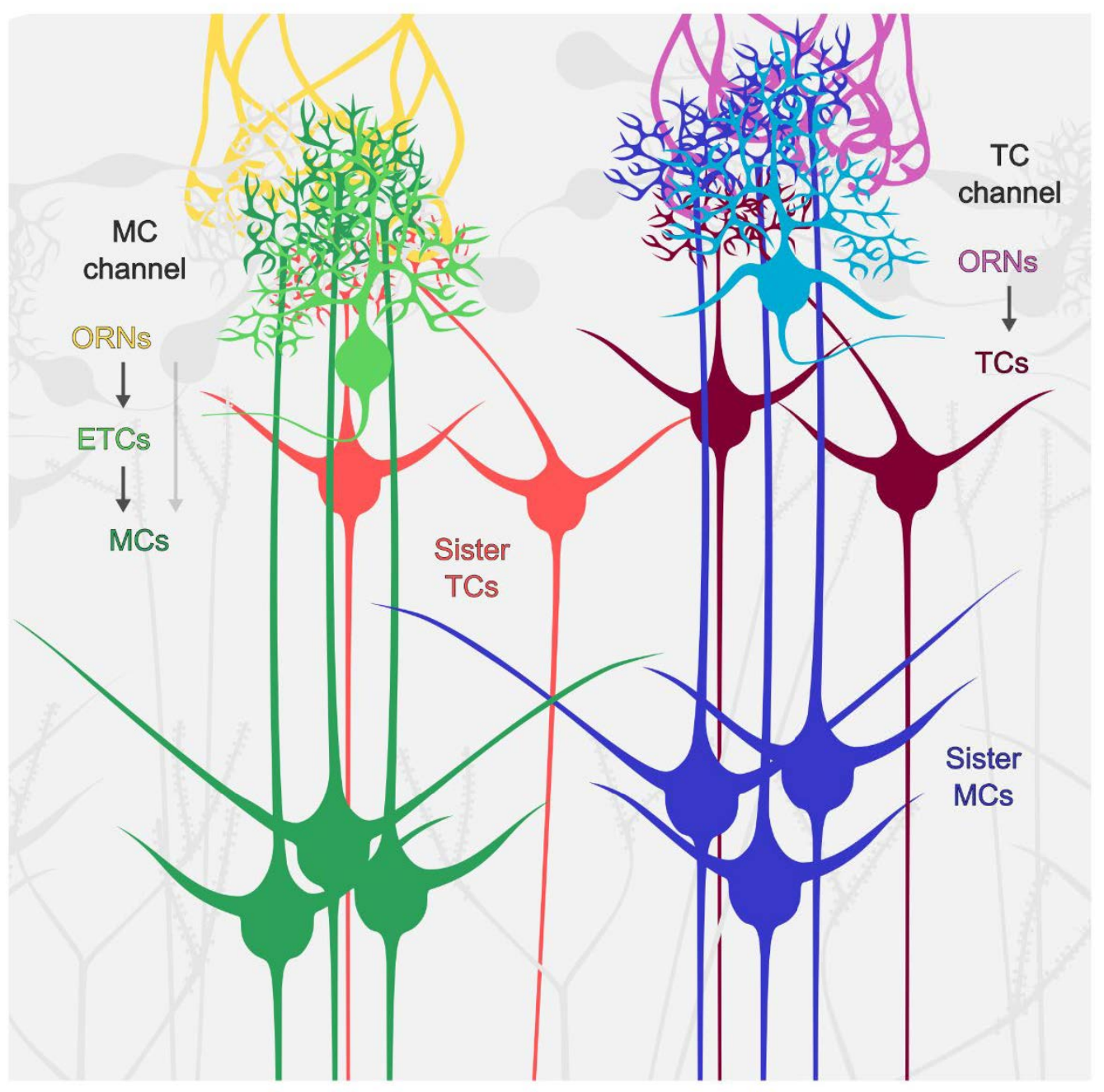

Schematic 9 MCs and TCs as parallel odor information processing channels

Odor information from different glomeruli (glomerulus 1: yellow, glomerulus 2: magenta) is conveyed via parallel MC and TC channels towards higher brain centers. The TC channel and the ETC-MC channel decompose glomerular input and can encode for different temporal or spatial aspects of odor information. TCs convey direct ORN input. Despite the existence of direct ORN input to MCs, ETCs mainly drive MC output via feed-forward excitation. MCs and TCs associated with one glomerulus are referred to as sister-MCs/TCs

\section{Neuromodulation between glomerular modules - lateral inhibition}

Granule cells (GCs) sheer number and their ability to inhibit several non-sister MTCs via reciprocal synapses, made them central candidates in early models of interglomerular lateral inhibition (Isaacson and Strowbridge, 1998; Rall et al., 1966; Urban and Sakmann, 2002). Their importance for olfactory coding is undeniable and their dynamic repertoire of inhibitory action ranges from sculpting individual MTC output, to coordinating entire neuronal ensembles' activity across the OB (Egger and Urban, 2006; Schoppa and Urban, 2003). While they play an essential role in early olfactory discrimination (Abraham et al., 2010), recent findings point to different mechanisms of GC lateral inhibition, than proposed in earlier studies in analogy to the retinal circuitry (DeVries and Baylor, 1993; Mori and Shepherd, 1994). The classical 'winner takes it all' model proposed a sharpening of the odor representation by lateral suppression of less strongly activated glomerular modules through strongly activated glomerular modules (DeVries and Baylor, 1993; Mori and Shepherd, 1994). This competition for odor representation space was mostly contributed to lateral inhibition 
through GCs and has been replaced by more modern approaches like 'winnerless competition' (Laurent et al., 2001). Lateral inhibition between glomerular modules seems to be mostly executed in the GL and not necessarily follow simple center-surround lateral inhibition logic (Aungst et al., 2003; Cavarretta et al., 2016; Economo et al., 2016). Interglomerular lateral inhibition acts on the level of MC or TC tufts and involves superficial short axonal cells indirectly inhibiting activity of other glomeruli: The excitatory superficial short axonal cells (SSACs) receive glomerular input from ETCs and can activate inhibitory PGCs of several distant glomeruli via their axons (Aungst et al., 2003; Banerjee et al., 2015; Whitesell et al., 2013). Economo and co-workers could show, that odor stimulation leads to specific, non-random patterns of inhibition and excitation on the glomerular array (Economo et al., 2016). Selective, lateral inhibition of glomerular modules was proposed to be less dependent on the position of the glomerular modules (center-surround inhibition) but more related to the actual molecular features detected by the involved glomeruli (Economo et al., 2016).

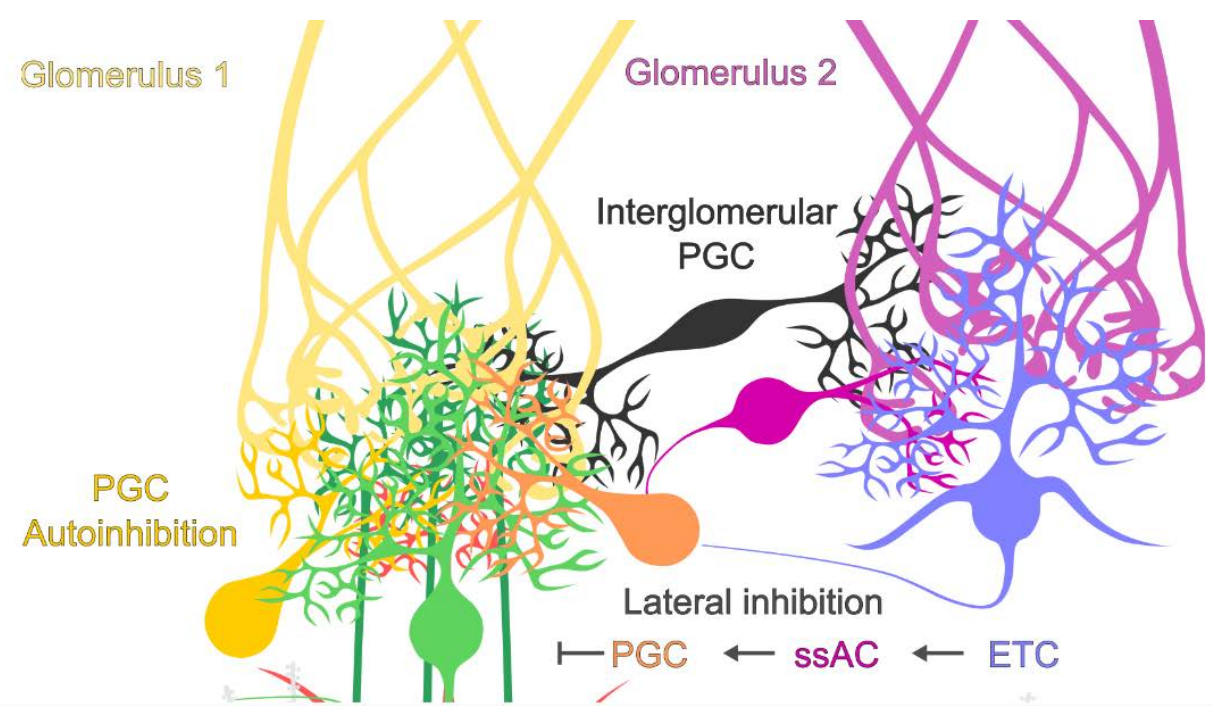

Schematic 10 Intra- and inter-glomerular neuromodulation in the GL

On the glomerular level, PGCs can exert autoinhibition onto the associated glomerular modules. The activation of individual glomerular modules can also lead to lateral inhibition of other glomerular modules. ETCs can excite superficial short axonal cells (sSACs) that subsequently trigger PGCs' inhibitory action onto other modules via their short axons. PGCs that project their dendrites into more than one glomerulus can also be involved in interglomerular inhibition.

PGCs that project their dendrites into more than one glomerulus can also be involved in interglomerular inhibition There is good evidence that GCs' effect on slow odor induced inhibition is not as substantial as inhibition on the GL level. Especially the observed suppression of spontaneously active glomeruli is unlikely to be caused by GC circuitry (Economo et al., 2016; Fukunaga et al., 2014). Lateral inhibition by GCs seems to be more involved in fast oscillatory synchronization of TC or MC output channels (Cavarretta et al., 2018; Fukunaga et al., 2014) and temporal decorrelation of MC output activity together with cortical feedback (Gschwend et al., 2015; Otazu et al., 2015). 


\section{Population coding in AMCs and glomerular modules in the AOS}

Apart from a few studies using multisite electrodes (Kahan and Ben-shaul, 2016; Tolokh et al., 2013) there are no comprehensive in vivo recordings of AMC ensemble activity. The group of AMCs is very heterogeneous and population activity patterns could only be derived from in vitro studies (Gorin et al., 2016; Kahan and Ben-shaul, 2016; Yoles-Frenkel et al., 2018). Extensive calcium imaging studies, as performed by Hammen et al. 2014, have not been done so far in the postsynaptic network of the AOB (Hammen et al., 2014).

\section{AMC cross channel integration instead of labeled line}

The fundamentally different wiring logic of the AOS makes a direct comparison to coding mechanisms discussed in the MOS difficult. AMCs usually receive excitatory input from several glomeruli via their multiple apical tufted dendrites (Takami and Graziadei, 1991; Urban and Castro, 2005; Yonekura and Yokoi, 2008). Given their multi-glomerular innervation, at least two different wiring strategies of AMCs with their parental glomeruli exist: In the homotypic configuration, an AMC receives input of VRNs expressing the same VR (Del Punta et al., 2002). In the heterotypic wiring configuration, AMCs innervate multiple glomeruli formed by different VRN species (Wagner et al., 2006). While this means that AMCs potentially integrate odor information of different receptors, the wiring was proposed to be selective heterotypic and related to amino acid sequence similarity between the V1Rs (Wagner et al., 2006). A combination of both wiring strategies could also be possible. It was suggested that in contrast to MTCS, AMCs encode for information of a set of molecular structures rather than single molecules (Wagner et al., 2006). The seemingly 'redundant' input of several glomeruli to AMCs was hypothesized to be a way of detecting particular molecular blends with behavioral relevance (Hammen et al., 2014; Wagner et al., 2006).

\section{AMC modules self- versus lateral inhibition}

In the $\mathrm{MOB}$, odor information is carried by spatio-temporal activity patterns of glomerular modules, each associated with one particular OR and glomerulus. The essential role lateral inhibition plays between MOB glomerular modules (Aungst et al., 2003; Cavarretta et al., 2016; Economo et al., 2016) might not be as pronounced in the AOB (Mori, 1987). Periglomerular cells are sparse in the AOB and mostly GABAergic (Mugnaini et al., 1984; Quaglino et al., 1999). In contrast to the MOB, there is no well-defined shell of PGCs surrounding AOB glomeruli (Meisami and Bhatnagar, 1998; Mugnaini et al., 1984). Few is known about their function and detailed connectivity, but the circuitry is supposed to be simpler than in the MOB: PGCs form reciprocal dendro-dendritic synapses with AMC tufts and are supposed to operate via feedback-inhibition (Mori, 1987). GCs of the AOB, like their counterparts in the MOB, form dendro-dendritic reciprocal synapses with AMCs (Hayashi et al., 1993; Jia et al., 1999; Taniguchi and Kaba, 2001). The silencing or 'gating' of AMCs by GCs was supported by studies investigating GABAergic inhibition on AMCs in memory formation experiments (Brennan and Keverne, 2015; Hayashi et al., 1993; Kaba et al., 1994). 
Physiological recordings from both PGCs and GCs will be necessary to prove, whether 'selfinhibition' is indeed the major type of AOB inhibitory neuromodulation on AMCs.

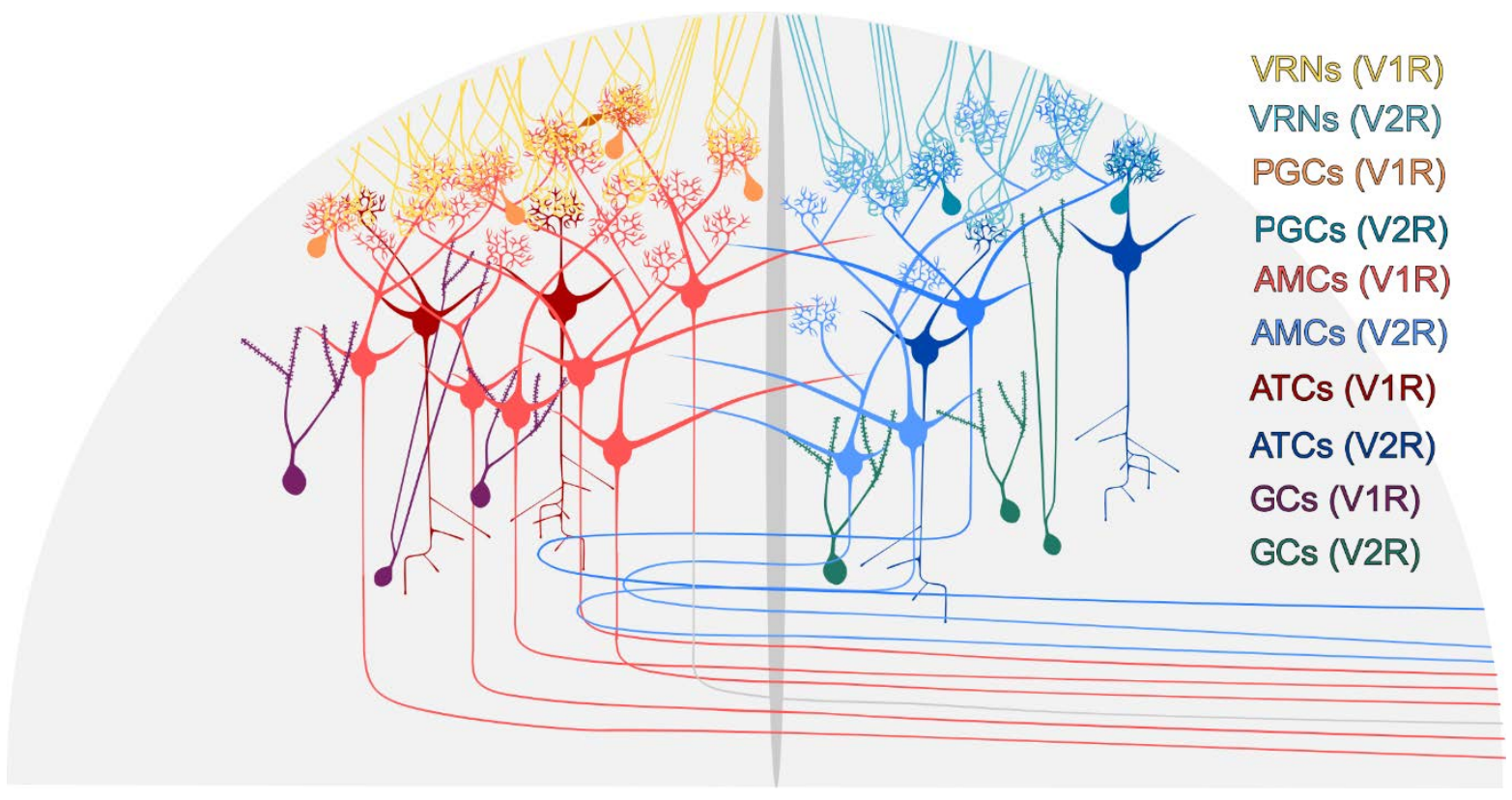

Schematic 11 Neuronal network organization of the AOS

Schematic summary of neuronal components making up the AOB neuronal networks. VRN species form multiple glomeruli. Their VR subtype (V1R or V2R) dictates which anatomical domain of the AOB they innervate. Both $A O B$ networks receive their input from VRN axons in the GL. The olfactory glomeruli are relay stations of presynaptic odor input to the excitatory projection neurons (AMCs) that convey odor information to the higher brain centers via their axons. Inhibitory interneurons modulate and sculpt the activity of projection neurons on the glomerular level (PGCs) and the level of the secondary dendrites (GCs). ATCs are excitatory interneurons that receive input of one glomerulus and project their axon collaterals to local targets in the AOB.

\section{AIM OF THE THESIS}

The main goal of my thesis was to investigate the wiring and odor information processing in the lateral olfactory subsystem of larval Xenopus laevis, especially on the glomerular and MTC level.

Regarding the wiring, the main aim of my work was:

1) to map the distribution of ORNs in the MOE that innervate the lateral glomerular cluster

2) to characterize the MTC population in terms of morphological subtypes, glomerular connectivity and neuronal marker expression

On the level of odor information processing my major goals were:

1) to characterize the postsynaptic glomerular odor map of the LC to amino acid and sulfated steroid stimuli

2) to analyze the distribution and odor tunings of glomeruli in the LC

3) Compare odor tuning of glomeruli to JGC and MTC somata to get insight into the glomerular module organization in larval Xenopus

The investigation of an evolutionary 'intermediate stage' olfactory subsystem between fish and mammals had the aim to help understand, how olfactory system circuitry evolved and adapted to the specific requirements of the individual species. 


\section{METHODS}

\subsection{MethodS: THEORETICAL BACKGROUND}

\subsubsection{Basics of Fluorescence and Fluorescence Microscopy}

Fluorescence Microscopy has revolutionized life sciences research. Fluorescent dyes or genetically encoded fluorescent proteins have enabled us to 'spy on' molecular and physiological processes to an extent hardly any other method available has (Tsien, 2005). Fluorescence is a property of individual molecules that are capable of absorbing photons. Absorption of a photon can lift electrons of those fluorophores from their ground state to electron orbitals of higher energetic level (Jablonski, 1933; Lichtman and Conchello, 2005). This excited state lasts only several femtoseconds, followed by internal conversion processes on energetic meta-levels. Once the electron returns into its ground state, the energy is emitted in the form of a photon of higher wavelength (Jablonski, 1933; Lichtman and Conchello, 2005; Stokes, 1852). This process is called fluorescence and happens in the nanoseconds range. The higher wavelength (lower energy) of the emitted photon is a result of the partial internal conversion of the electron's energy and vibrational relaxation before returning to the ground state level (Lichtman and Conchello, 2005). This difference between the energy/wavelength of the photon exciting the fluorophore and the one it emits is also called 'Stokes-shift' and forms the basis of most fluorescence microscopy methods since it allows separation of the emission from the illumination wavelength (Stokes, 1852; Lichtman and Conchello, 2005). Fluorescence microscopy via epi-illumination is one of the simpler microscopy methods. White excitation light is generated in light sources like halogen/arc lamps and sent into the microscope via light guides. Filter cubes are essential modules of these microscopes that are composed of an excitation filter, an emission filter, and a dichroic mirror. They represent an elegant way of directing excitation light of particular wavelengths through the microscope's objective to the sample and are at the same time capable of selectively transmitting emitted fluorescence from the sample through the objective to the ocular or camera (Schematic 12; Lichtman and Conchello, 2005). A dichroic mirror reflects the excitation wavelengths but is transmissive for the fluorescence emission wavelengths. The emission filter in the cube increases the selectivity of the microscope by only transmitting the wished wavelengths of fluorescent light passing through the dichroic mirror (Lichtman and Conchello, 2005). Fluorescence is most efficiently emitted from fluorophores in the focal plane of the objective. While the photon density is highest in the focal plane, fluorophores in different planes of the light-path can also be excited to a lesser extent. This out-of-focus emission leads to a loss of information about the fluorescent signal's origin in the z-dimension. Together with effects like light scattering and interference in biological samples represents one of the major limitations of this method (Claxton et al., 2006; Lichtman and Conchello, 2005). 
Confocal microscopy, especially laser scanning confocal microscopy, has significantly improved the resolution of fluorescence microscopy to the level of subcellular structures or even larger molecules (Claxton et al., 2006). By using several lenses and an adjustable pinhole for the emission wavelengths, light originating from off-focus planes can be excluded from the detected fluorescence emission (Wilson and Sheppard, 1984). Instead of continuous white light for fluorophore excitation, in laser scanning confocal microscopes, laser beams of single wavelengths raster-scan across the sample, directed by Galvano mirrors (Claxton et al., 2006). A photomultiplier collects every scanned voxel's emitted photons. The photon count was assigned as an intensity value to the voxel's position. By using piezo-elements or motors, the objective can be moved in z-direction in minimal steps. Together with the confocality defined by the pinhole, this allows plane-wise sectioning of biological samples at high spatial resolution along all axes (Claxton et al., 2006). By using multiple lasers of different wavelengths, suitable lenses and filters, multi-color imaging of entire tissue volumes are possible with this technique (Claxton et al., 2006). Spectral fingerprinting' of the emission light from simultaneously excited different fluorophores can be spectrally decomposed by diffraction grating and collected by several bins each containing a photomultiplier. Emission light from different fluorophores can be spectrally decomposed by diffraction-grating and collected by several photomultipliers. This method of spectral fingerprinting allows a way higher resolution of the fluorescence emission wavelength spectrum (at $\sim 10 \mathrm{~nm}$ steps) than regular filter cubes. Moreover, it enables simultaneous multi-color imaging and the distinction of multiple different fluorophores (Zeiss; http://zeiss-campus.magnet.fsu.edu/ articles/spectralimaging/ introduction.html).

The introduction of multiphoton microscopy to neuroscience research has boosted the progress in both structural and functional imaging of the nervous system (Denk and Svoboda, 1997; Yang and Yuste, 2017). Two-photon excitation can occur if two photons of half the energy that is required to lift an electron to the excited state hit a fluorophore virtually simultaneously (Denk et al., 1990; Göppert-Mayer; Peticolas et al., 1963). This two-photon effect happens very sparsely compared to one-photon excitation and thus requires high laser powers with mode-locked, pulsed laser light, for instance, from Titan-sapphire lasers (Denk et al., 1990; Spence et al., 1991). The sparseness of the two-photon effect comes with a major advantage on the other hand: In out of focus planes, the photon density is far too low to excite fluorophores efficiently. Consequently, pictures taken by a multiphoton microscope are 'intrinsically confocal' (Helmchen and Denk, 2005). The ability of pulsed infrared light to travel through biological samples several hundred micrometers without substantial interference with the tissue allows taking 3D image stacks of samples with superior resolution in-depth compared to confocal microscopy (Denk and Svoboda, 1997; Helmchen and Denk, 2005; Svoboda et al., 1997). Acquisition of 3D image-stacks in raster-scanning mode is possible as in confocal microscopy (Göbel and Helmchen, 2012; Helmchen and Denk, 2005). By using mirrors that vibrate at high frequencies (resonant laser scanning) instead of the 
Galvano-mirrors, the sample can be scanned at an even higher acquisition rate (in our microscope up to $400 \mathrm{~Hz}$; Nikon A1R MP). In this work, functional recordings of neuronal activity were performed using this resonant scanning mode. Samples where the fine morphological features of cells mattered were imaged in laser scanning mode.

\section{One photon \\ fluorescence}
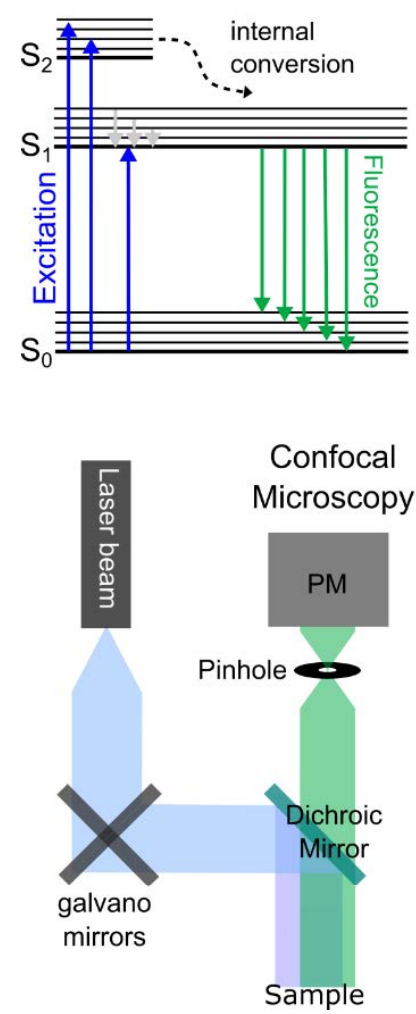

Two photon

fluorescence
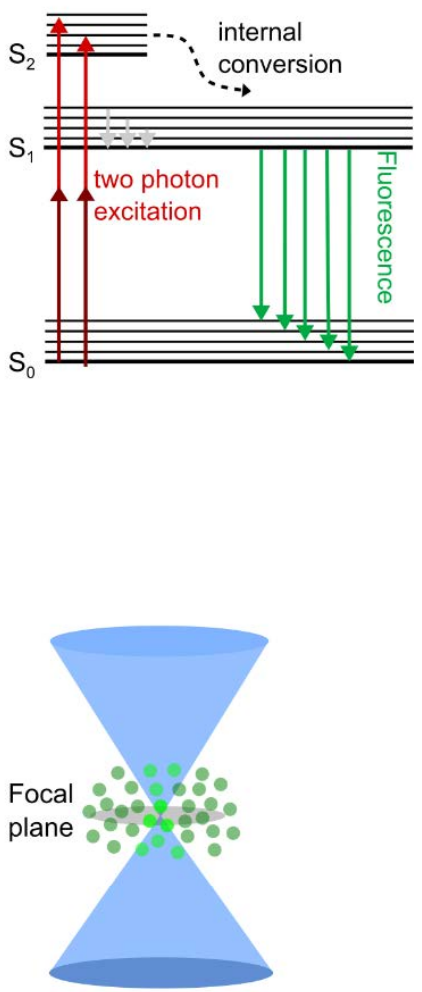

Epifluorescence Microscopy
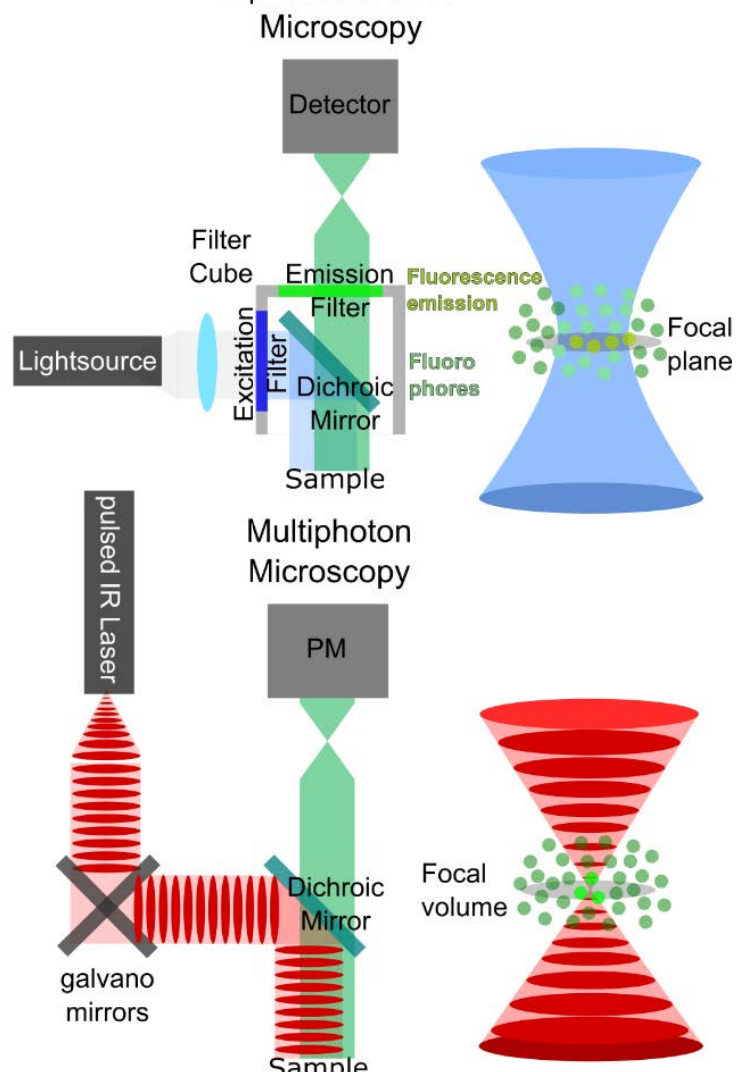

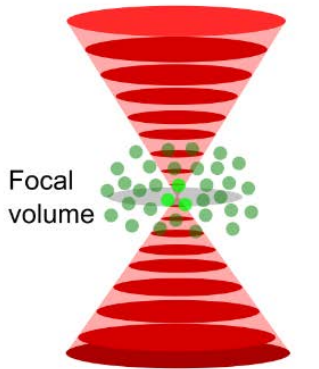

Schematic 12 Principles of epifluorescence, confocal and multiphoton microscopy

In epifluorescence microscopy, excitation light of particular wavelength is created (for example via white light passing through an excitation filter), deflected by a dichroic mirror and focused via lenses to excite fluorophores (green dots) in a focal plane of the imaged sample (yellow dots, upper third, left). Fluorophores that are not in the focal plane but in the cone of the light beam, can get excited, less probably than in the focal plane though (light green dots, upper left). Upon photon absorption (f. ex. blue light), the fluorophores emit fluorescence as lower energy photons (f. ex. green light). Fluorescent light reenters the objective, passes the dichroic mirror and an emission filter and can be detected in the ocular or by a camera. In confocal microscopy the light source is usually a laser beam of narrow wavelength spectrum, that raster-scans the focal plane using moveable mirrors and excites fluorophores. Out of focus fluorescence from non-focal fluorophore excitation can be filtered out by a pinhole in the light-path before the photomultiplier (PM). In multiphoton microscopy, pulsed infra-red laser light is used to excite fluorophores taking advantage of the two photon-effect. Two photons of half the energy as the excitation wavelength needed to excite the fluorophores can lift electrons to the high energy state if they arrive virtually simultaneously. This effect is very improbable and needs high photon densities that only occur in the focal plane, thereby granting multiphoton microscopy its 'confocality' The emitted fluorescence of lower wavelength light is collected via sensitive PMs.

\subsubsection{Calcium imaging and AM dyes}

Calcium ions are, without a doubt, among the most important second messengers in living organisms and are involved in a plethora of physiological processes like development, muscle contraction, and neuronal signaling (Berridge, 1993; Giorgi et al., 2018). Neurons invest a considerable amount of energy in the maintenance of low calcium concentration in their cytoplasm in comparison to the extracellular space or the cellular organelles (Clapham, 
2007; Giorgi et al., 2018). The resulting high $\mathrm{Ca}^{2+}$ gradient across the membranes can be used by neurons to trigger depolarization, calcium-dependent signal transduction pathways, or synaptic vesicle release through $\mathrm{Ca}^{2+}$ influx into the cytoplasm (Giorgi et al., 2018). The concentration of free $\mathrm{Ca}^{2+}$ in the cytoplasm of neurons and its spatio-temporal dynamics are major readout parameters of neuronal activity in modern neuroscience research (Giorgi et al., 2018). Nowadays, a plethora of different probes and tools to monitor calcium dynamics in neurons exist, offering in vitro and in vivo imaging applications with high spatial and temporal resolution (Lin and Schnitzer, 2016; Yang and Yuste, 2017). Chemical calcium indicators have hugely contributed to our understanding of neuronal signaling. Even with the rise of genetically encoded biosensors, they still represent valuable tools for research questions that require accurate knowledge of calcium concentration and binding dynamics (Paredes et al., 2009). In this work, I mainly used the high affinity, single wavelength calcium indicator Fluo4 (Thermo-Fisher; Invitrogen ${ }^{\mathrm{TM}}$ ). Its fluorescence quantum yield increases drastically once its carboxy-groups form a transient chelate complex with free calcium ions (Tsien, 1981). The change in fluorescence quantum yield can be detected as an increase in green fluorescence emission under constant excitation conditions. Instead of its one-photon excitation maximum at $488 \mathrm{~nm}$, I used $800 \mathrm{~nm}$ wavelength laser light to excite Fluo-4 under the multiphoton microscope. One powerful approach used in the field has been to add acetoxy-methyl (AM) groups to the carboxyl-groups of the calcium indicator. The addition of those groups turns the negatively charged chemical calcium indicator neutral and facilitates its uptake into neurons at the same time avoiding extrusion or compartmentalization (Tsien, 1981). The AM-esters of the chemical calcium indicator are less hydrophilic, so that passive diffusion through the plasma membrane is possible. Intracellular esterases hydrolyze ester bonds and are present in almost all living cells. Consequently, the chemical calcium indicator returns to its functional, hydrophilic form and gets trapped inside the cell (Tsien, 1981). For my calcium imaging experiments, I used Fluo-4 AM dye (Thermo-Fisher; Invitrogen ${ }^{\mathrm{TM}}$ ) to load neurons in the OB of larval Xenopus via multi-cell bulk loading (MCBL; Garaschuk et al., 2006).

\subsubsection{Neuronal tracers}

To understand neuronal networks, it can be crucial to label neuronal populations and their projections selectively. Already in the early studies by Golgi and contemporaries, the sparse, seemingly stochastic labeling of individual neurons served to get first hints about neuronal populations and their connectivity (Figueres-Oñate et al., 2014; Nassi et al., 2015). It was not until the introduction of tracer molecules like the protein horseradish-peroxidase (Kristensson and Olsson, 1971; LaVail and LaVail, 1972) that entire populations of neurons could be traced via their processes. Horseradish peroxidase can be taken up by axonal terminals and transported towards the neurons' somata (Kristensson and Olsson, 1971). By conjugating horseradish peroxidase to plant lectins like wheat germ agglutinin (WGA), neuronal tracing was improved significantly (Gonatas et al., 1979). WGA is a protein that can bind to polysaccharides containing $\mathrm{N}$-acetyl glucosamine residues (Bains et al., 1992). Upon binding 
to sugar epitopes exhibited on neuronal membranes, it is taken up via endocytosis. It can be transported in vesicular structures through neuronal processes in either anterograde or retrograde manner (Broadwell and Balin, 1985). One of the most intriguing features of this tracer represents its capability to cross synapses and thus label interconnected neurons (Broadwell and Balin, 1985; Yoshihara et al., 1999). Its efficient uptake by any neurons that exhibit suitable carbohydrate epitopes and the fast transport into both directions make WGAcoupled fluorophores valuable tracers for neuronal populations (Reeber et al., 2011; Tsuriel et al., 2015).

\subsubsection{Electroporation of dextran coupled fluorophores into neurons}

Dextrans are large poly-carbohydrates that can get coupled to fluorophores. The large, charged tracer molecules can neither cross cellular membranes nor be degraded easily inside the cell. In some cases, dextran-amines were reported to be taken up by neurons via unknown mechanisms (Reiner et al., 2000). Their delivery into cells often depends on the temporary disruption of the plasma membrane barrier (Chen et al., 2006a; Haas et al., 2002). Electrical fields are capable of transiently permeabilizing cell membranes. Voltages surpassing particular membrane specific thresholds, lead to the formation of short-lived pores that can enable the passage of macromolecules (Chen et al., 2006a; Ho and Mittal, 1996). Besides the diffusion-limited movement through those transient pores, charged molecules can be actively transported into cells via the applied electrical field (Neumann et al., 1999). Micropipette tip diameters of only a few micrometers restrict the electroporation to single or few cells. In contrast, dextran-coupled dye injection and application of external electrical fields via plate or wire electrodes can lead to the labeling of entire neuronal populations (Haas et al., 2002). The electroporated, dextran-coupled fluorophores can be visible for days up to several weeks in Xenopus laevis (Dittrich et al., 2016; Haas et al., 2002; Hassenklöver and Manzini, 2013).

\subsection{Methods: EXPERIMENTAL PROTOCOLS}

\subsubsection{Animals and animal husbandry}

All scientific procedures on living animals were approved by the regional board (RP Giessen Az: V54-19c2015h01 GI 15/7; Niedersächsisches Landesamt für Verbraucherschutz und Lebensmittelsicherheit, Oldenburg, Germany, Az: 16/2136) in accordance with the German animal welfare law and the European legislation for the protection of animals used for scientific purposes (2010/63/EU). I used Xenopus laevis wild-type (pigmented or albinos) or transgenic tadpoles of stages 51-54 (Nieuwkoop and Faber, 1994) for all experiments. Wildtype (NASCO strain) and transgenic tadpoles (European Xenopus Resource Center; EXRC) 
Portsmouth, UK, Line: EXRC \#202; Xla.Tg(tubb2b:Katushka;cryga:Venus) were reared at $22{ }^{\circ} \mathrm{C}$ in our breeding facility in Göttingen/Gießen and fed daily with algae (Chlorella, Dohse Aquaristik $\mathrm{GmbH}$, Bonn, Germany). Animals lived in aquaria under $12 \mathrm{~h}$ dark/light cycles.

\subsubsection{Solutions}

Frog ringer consisted of (in millimolar): $98 \mathrm{NaCl}, 2 \mathrm{KCl}, 1 \mathrm{CaCl} 2,2 \mathrm{MgCl} 2,5$ glucose, 5 sodium-pyruvate, 10 hydroxyethyl piperazineethanesulfonic (HEPES), $230 \mathrm{mOsmol} / \mathrm{l}, \mathrm{pH} 7.8$ (All chemicals were purchased from Sigma). I dissolved odorants as concentrated stock solutions $(10 \mathrm{mM})$, aliquoted and stored them at $-20^{\circ} \mathrm{C}$. Odor solutions of $100 \mu \mathrm{M}$ concentration were freshly prepared before each experiment from aliquots (only unthawed and used once). I used odorant solutions of the individual L-amino acids (Sigma-Aldrich, HPLC grade), arginine $(R)$, histidine $(H)$, isoleucine $(I)$, leucine $(L)$, lysine $(K)$, methionine $(M)$, phenylalanine $(F)$, tryptophan $(\mathrm{W})$, valine $(\mathrm{V})$ and odor mixtures of amino acids with basic/aromatic $(H, K, R, F, W)$, long-chain-neutral $(M, L, I, V)$ or small-chain-neutral $(G, T, S$, C) residues. I moreover used sulfated steroids as odorants in two mixtures called $P$ - and $E$ mix (as described in Sansone et al., 2015). A mixture of alcohols, ketones and aldehydes ( $\alpha$ Terpineol, $\beta$-ionone, $\beta$-phenylethylalcohol, $v$-phenylpropylalcohol and citral; Sigma-Aldrich) was used as a negative control for the lateral glomerular cluster (Gliem et al., 2013).

\subsubsection{Calcium indicator and Calcein AM solutions}

Fluo-4 and Calcein AM dye mixes (Thermo Fisher) for injection into the OB of the wholemount preparation were prepared the same day of the experiment. $50 \mu \mathrm{g}$ of Fluo- 4 or Calcein AM were dissolved in $5 \mu \mathrm{l}$ of DMSO (Sigma-Aldrich). Sequentially, $10 \mu \mathrm{l}$ of Pluronic F-127 (Sigma-Aldrich) and $35 \mu \mathrm{l}$ of frog ringer were added to the solution. After $1 \mathrm{~min}$ of centrifugation (at 16,100 relative centrifugal force) I added $3 \mu \mathrm{l}$ of MK571 (50 $\mu \mathrm{M}$, Alexis Biochemicals) and $0.3 \mu$ cascade blue dextran (Thermo Fisher) to the supernatant. MK571 was used to inhibit multi-drug transporters that might lead to cellular extrusion of the AM dyes (Manzini and Schild, 2003b). The cascade-blue dextran allowed visualization of the injected dye bolus in the tissue, using fluorescent illumination.

\subsubsection{Bulk electroporation of the main olfactory epithelium}

I anesthetized tadpoles of developmental stages 51-54 in $0.02 \%$ MS-222 (ethyl 3aminobenzoate methanesulfonate; Sigma-Aldrich; $\mathrm{pH}$ : 7.6) up to 5 min until the complete absence of responsiveness. The animal was placed on a wet paper towel on a petri-dish and kept moist throughout the entire procedure. I removed excess water in the proximity of the olfactory organs with a paper tissue until there was only residual liquid left in the nostrils. A desiccated crystal of dextran-coupled fluorophore was carefully placed into the nostrils with fine forceps, where it dissolved in the remaining moisture. I generated the dye crystals beforehand by dissolving the fluorophore-coupled dextran at a concentration of $3 \mathrm{mM}$ in frog ringer (Oregon-Green 488 BAPTA-1 dextran; 10.000 MW; Thermo Fisher) and then pipetting tiny droplets of the solution on parafilm. Crystals formed after $30 \mathrm{~min}$ at room temperature. 
Several dye crystals were dissolved in the remaining moisture of the nasal cavities until the whole cavity was saturated with the dye. I carefully positioned two platinum wire electrodes (diameter: $0.2 \mathrm{~mm}$ ) in a way that one electrode was submerged in the dye-filled nostril and the other contacted the animal's cheek laterally of the ON (Schematic 13). Via a voltage pulse generator (ELP-01D, NPI Electronics; additional capacitor connected in parallel: Domoport, $3 \mu \mathrm{F})$ four to six trains of 3 square pulses (15-20 V, $500 \mathrm{~ms}$ duration and $25 \mathrm{~ms}$ delay) were applied in alternating polarity (larvae of stages. 51-52: $15 \mathrm{~V}$ four trains; larvae of stages 5354: $20 \mathrm{~V}$, six trains). The voltage pulses were observable by gas formation due to hydrolysis at the electrodes. Following electroporation, animals were left to awaken from anesthesia. After I assessed their health status, I put them into a big beaker filled with tap water to recover for 24 to $48 \mathrm{~h}$. On the day of the experiment, animals were not fed and kept in the dark to avoid photobleaching of the calcium indicator.

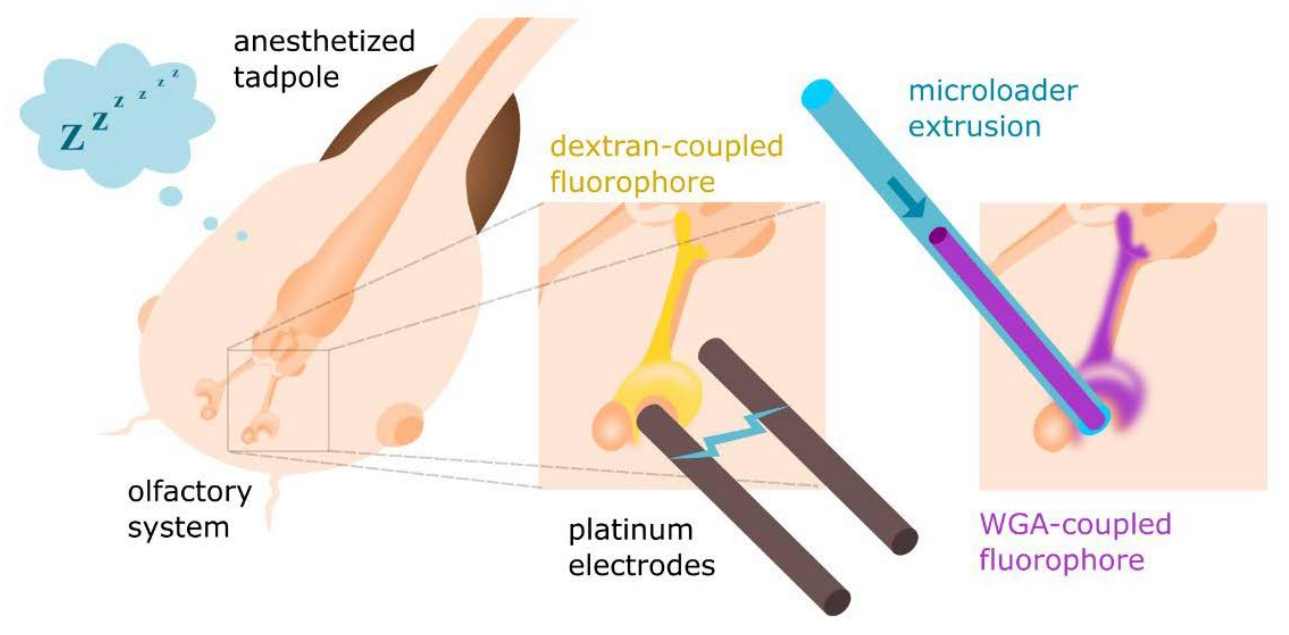

Schematic 13 Bulk electroporation of dextran-coupled fluorophores and WGA staining of glomerular clusters

ORN axonal projections can be labeled by dissolving dextran-coupled dyes in the nostrils of anesthetized tadpoles and subsequent application of electrical pulses via wire electrodes. The transiently permeabilized neuronal plasma membranes allow the passage of the dextran-coupled dyes into ORNs where they get transported to the axon terminals. WGA-coupled fluorophores can be directly administered into the nostrils of larval Xenopus laevis. The dye is endocytosed in minutes and gets transported in vesicular structures through the ORNs in anterograde and retrograde direction.

\subsubsection{Sparse MTC electroporation in the ventral OB}

One to two days after bulk electroporation of larval Xenopus olfactory mucosa with OregonGreen 488 BAPTA-1 dextran (MW 3.000, Thermo Fisher), I prepared 'whole-mount preparations' for sparse-cell electroporation experiments. Therefore, I anesthetized tadpoles for $5 \mathrm{~min}$ in MS-222, assessed proper anesthesia, and sacrificed them by severing their nervous system with two cuts, caudally and rostrally of the brain stem using fine scissors. A rectangular block containing the entire peripheral olfactory system and the bulb was cut out and pinned ventral side up to a silicone-filled petri dish with fine needles. I removed excess connective tissue and exposed the $\mathrm{OB}$ by carefully removing the superincumbent, cartilaginous, palatial tissue until the level of the ON with fine scissors. I mechanically fixed 
this whole-mount preparation in a recessed plastic recording chamber via a nylon-stringed platinum grid and covered it with frog saline to keep the tissue alive and moist.

The recording chamber was placed on the stage of an upright fluorescent stereomicroscope (Olympus BX51WI) equipped with the sparse-cell electroporation setup described in detail in (Weiss et al., 2018). I pulled micro-pipettes from borosilicate glass capillaries (outer diameter $1.5 \mathrm{~mm}$, inner diameter $0.86 \mathrm{~mm}$, length $100 \mathrm{~mm}$; Warner Instruments) with a horizontal pipette puller (P 1000, Sutter Instruments). I fabricated micro-pipettes with slim tapers and pipette resistances between 8-15 M $\Omega$. Micropipettes were filled with $4 \mu \mathrm{l}$ of the dextrancoupled fluorophore dissolved in frog ringer (3 mM; Alexa Fluor 488 / 555 / 594 or cascade blue, all $\sim 10.000$ molecular weight (MW); Thermo Fisher). I mounted the tracer-filled micropipettes to $\mathrm{a}$ head-stage (AP-1A-0.1MU, Axon instruments) attached to $\mathrm{a}$ micromanipulator (Scientifica, PatchStar). The AgCl-coated wire was partially submerged in the dye solution, and a reference electrode was put into the frog ringer on the recording chamber. The micropipette was carefully lowered into the liquid column between the waterimmersion objective and the tissue sample. I focused the pipette tip and lowered it further with the micromanipulator until the surface of the ventral bulb was visible. In addition to bright field illumination, I used fluorescent light from a coupled light source (AMH-200-F6S; Andor Technology-Oxford Instruments) to spot the green fluorescence of the ORN axons (labeled with Oregon-Green 488 BAPTA-1 dextran, MW 3.000, Thermo Fisher) to navigate the pipette tip towards the MCL. I penetrated the pipette tip along its axis into the MCL of the bulb using the virtual approach mode of the micromanipulator. Once I found cellular outlines of MTC somata of interest by bright field illumination, I approached the micropipette tip into proximity of the cell body. To label MTCs, I triggered trains of positive square voltage pulses with the single-cell electroporation device (voltage $50 \mathrm{~V}$, a pulse length of $300 \mu$ s and a frequency of $300 \mathrm{~Hz}$ for $500 \mathrm{~ms}$; Axoporator 800A, Axon Instruments / Molecular Devices). Upon successful electroporation, dye extrusion from the pipette tip and the outlines of the filled MTC soma and dendrites were quickly visible under fluorescent illumination. Consequently, I carefully withdrew the micropipette from the tissue sample. 


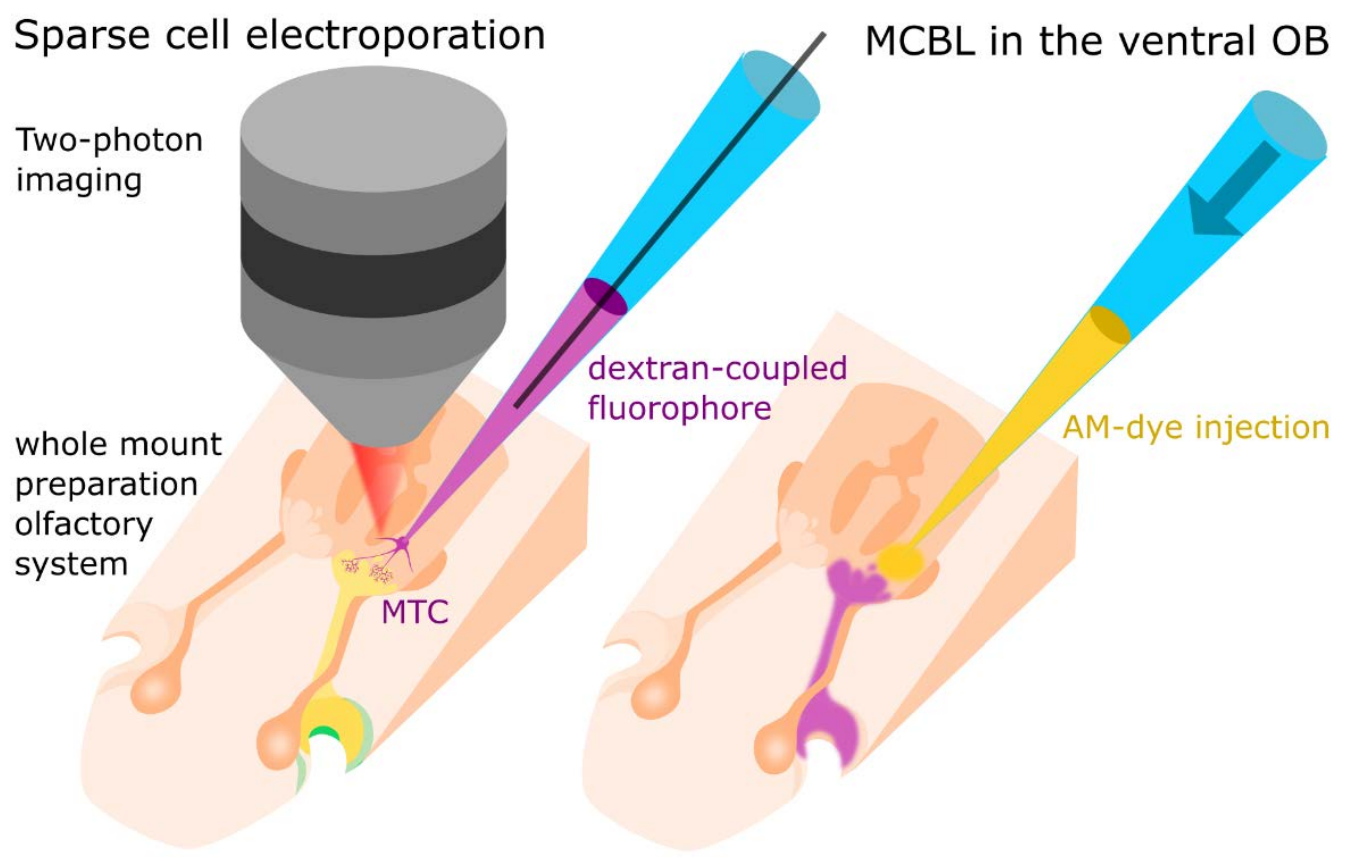

Schematic 14 Sparse cell electroporation and MCBL of Fluo-4 AM calcium indicator

Morphologies of MTCs and other neurons in the OB can be visualized by sparse cell electroporation. Dextrancoupled, fluorophore-filled micropipettes are used to load MTC somata in proximity of the pipette tip via trains of electrical pulses (left panel). For calcium imaging in entire populations of OB neurons, Fluo-4 AM can be injected into the ventral $\mathrm{OB}$ by pressure application (right panel). For both methods, prior ORN axonal stainings (fluorophore-coupled dextrans: yellow; WGA-coupled fluorophores: magenta) facilitate orientation in the OB.

\subsubsection{Retrograde tracing of ORNs with WGA-Alexa Fluor conjugates and neuronal positioning system}

For in vivo injections of fluorophore coupled WGA, I pulled borosilicate pipettes (outer diameter $1.5 \mathrm{~mm}$, inner diameter $0.86 \mathrm{~mm}$, length $100 \mathrm{~mm}$; Warner Instruments) with a long taper and high resistance (10-15 $\mathrm{M} \Omega$ ). The width of the pipette's opening was increased by carefully touching a moistened microfiber tissue. I assessed the quality of the resulting 'break tip' micro-pipettes under a microforge device (MF-830, Narishige Japan). Subsequently, suitable pipettes were loaded with 3-5 $\mu$ of WGA-Alexa Fluor conjugate solution $(2.5-10$ $\mu \mathrm{g} / \mu \mathrm{l})$. I vortexed the solution after unthawing and directly before loading to avoid aggregation of WGA-conjugates. I mounted the loaded glass pipettes to a micromanipulator and connected it to a Microinjector device (Femto Jet express, Eppendorf)

The following procedures were carried out under a stereomicroscope (Olympus SZX16; light source: X-Cite Series 120 Q, Lumen dynamics) either under bright field or fluorescent illumination. Albino tadpoles were anesthetized as described previously and placed on a moistened, silicone-filled petri dish. I used a scalpel to create a small incision into the animal's skin at the level of the midline between the two OB hemispheres (Schematic 15). Using fine scissors, I perforated the subjacent meninges to enable proper access to the OB for the 
micropipettes. I performed four subsequent injections of four different WGA-coupled fluorophores (WGA-Alexa Fluor 488, 555, 594, and 647; Thermo Fisher) at various locations in the rostral OB. Using the micro-manipulator, I carefully penetrated the micropipette's tip through the incision into the OB tissue. After I verified the proper positioning of the tip using fluorescent illumination, I gradually increased the continuous injection pressure on the microinjector until remote dye extrusion from the tip was observable. By increasing the constant pressure (50-100 $\mathrm{hPa}$ ) above this threshold, dye injection into the tissue occurred gradually. Once the dye spread sufficiently in its defined target region, the pressure was turned off, and the micropipette carefully withdrawn from the tissue.

I targeted four distinct sites in the $\mathrm{OB}$ and injected the different dyes that there was partial overlap between the injections similar as described by (Tsuriel et al., 2015) The four injections were performed, i) between the LC and the AOB (Alexa Fluor 488), ii) between the LC and medial cluster (Alexa Fluor 594), iii) between the ventral and dorsal medial cluster (Alexa Fluor 555) and into the dorsal OB (Alexa Fluor 647). Only animals with four proper injections were used for further experiments. After a successful injection, I closed the incision of the skin with tissue glue, and animals were put back into a beaker filled with tap water until they awoke from anesthesia. I assessed normal swimming behavior and tactile responsiveness before transferring the larvae to a big water-filled beaker. There they recovered for $24 \mathrm{~h}$ (in the dark to avoid photobleaching of fluorophores).

The next day, I anesthetized and sacrificed the animals as described previously. Subsequently, a rectangular tissue block containing the olfactory organs and bulb was cut out (Schematic 15; middle panels). The tissue block was then cut coronally at the level of the ON entering the OB. Using a vibratome (VT 1200 S, Leica), I cut the block containing the olfactory organs twice in intervals of $100-120 \mu \mathrm{m}$, once from the anterior side and once from the posterior side (Schematic 15; lower panels). This sectioning technique allowed me to 'unfold' the tissue block (See Schematic 15). This sectioning technique facilitated orientation and allowed me to scan all layers of the olfactory organ with a laser scanning confocal microscope from both sides (upright LSM 780, Zeiss). Before scanning, the OB was pulled out of its cavity and together with the unfolded tissue block of the olfactory organs, fixed with a nylon-stringed platinum grid in a recording chamber containing frog ringer.

I used the lambda scanning mode of the confocal microscope (LSM 780, Zeiss; Zen 2010 software) to acquire image stacks of spectral emission profiles of both the OB and MOE. The ventral and the dorsal side of the olfactory organ section was scanned. Laser wavelengths of 488, 514 (Argon), 561 (DPSS), and 633 (HeNe) lasers were used at fixed power to excite fluorophores in all samples of one animal equally. A 32-channel spectral detector collected fluorescence emission light in 8-10 nm wavelength bins (module of LSM 780, Zeiss). 


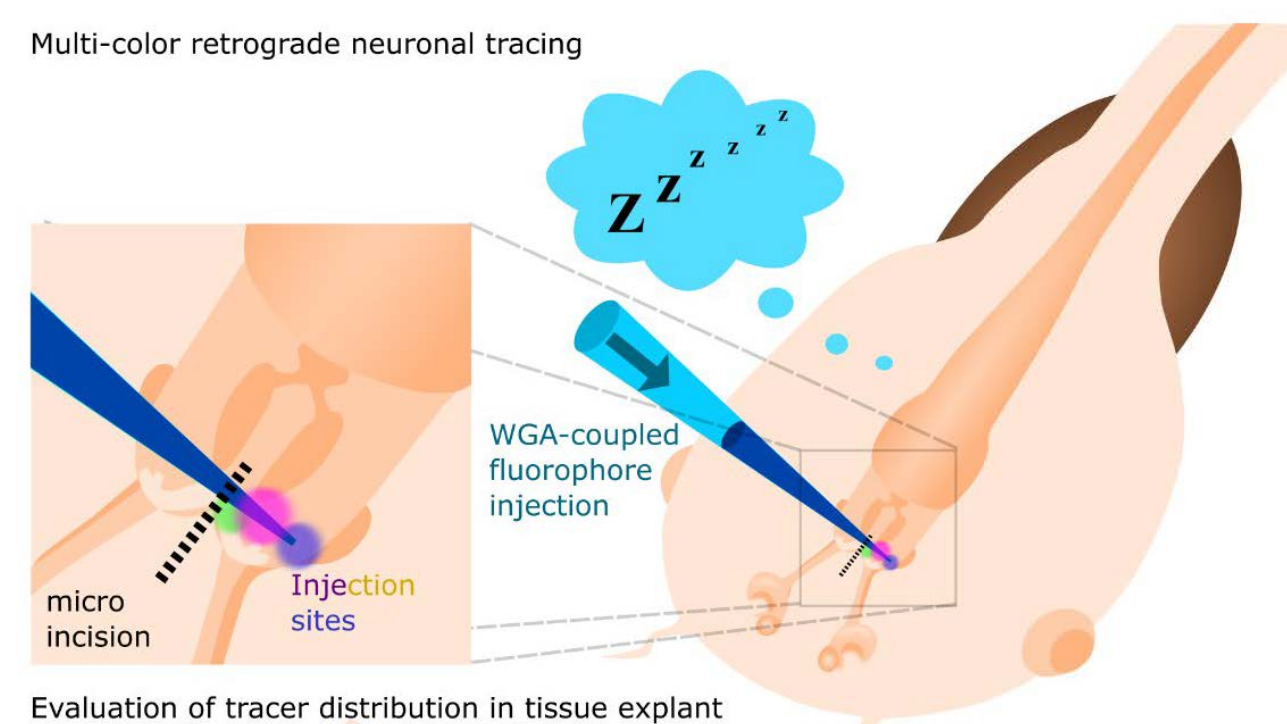

Evaluation of tracer distribution in tissue explant

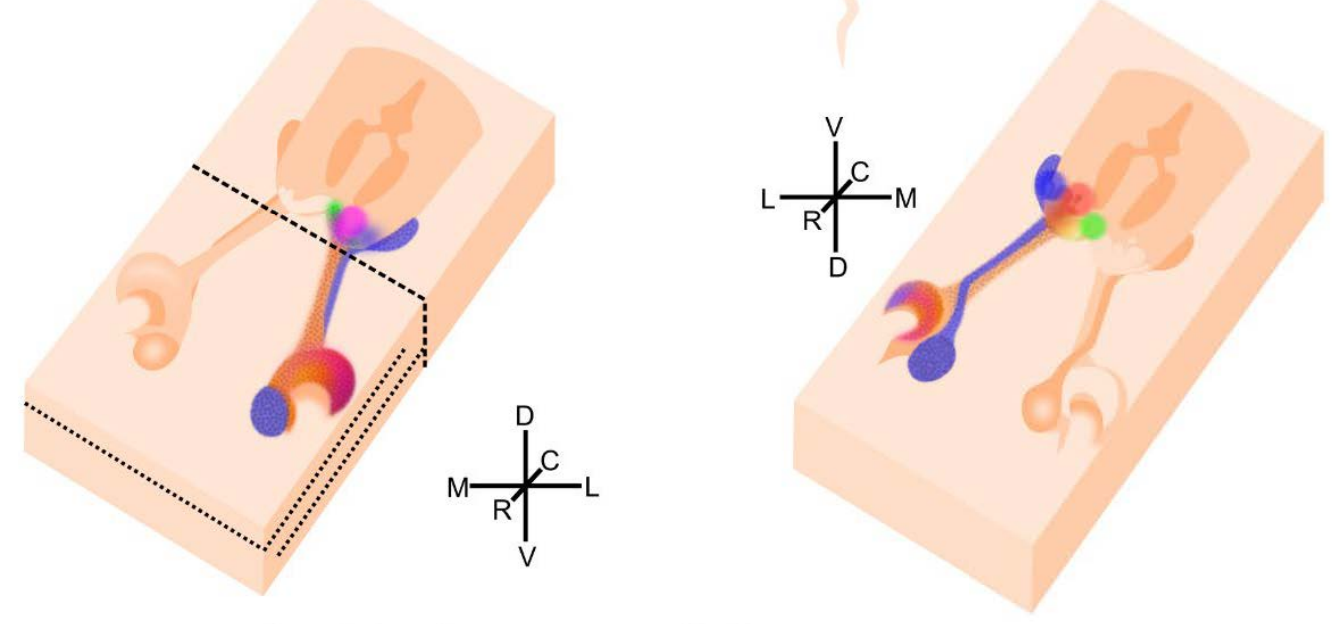

Tissue sectioning for whole olfactory organ and bulb imaging

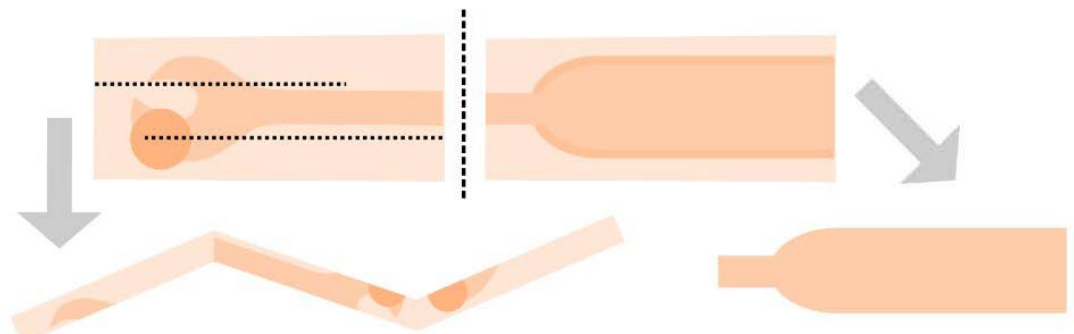

Schematic 15 Multi-color retrograde tracing of ORN axonal projections with WGA-coupled fluorophores

WGA-coupled fluorophores can be injected into the OB of anesthetized tadpoles through a surgical micro-incision. Differently colored tracers injected at different sites in the OB, get retrogradely transported by ORN axons to the olfactory organs in a few hours. The whole olfactory system explant can be split into the OB part and a block containing the olfactory organs. This block is sectioned in a way that the entire olfactory system can be scanned from both sides with confocal microscopy to image tracer distribution.

\subsubsection{Anterograde labeling of glomerular clusters via dextran coupled WGA}

I anesthetized albino tadpoles in 0,02\% MS-222 for at least 5 min until they were completely anesthetized and irresponsive. Subsequently, I transferred the animal to a petri dish covered in moist micro-fiber tissue (Kimtech) and removed excess water in and around the principal cavity with a micro-fiber tissue (Kimtech). I pipetted $250 \mathrm{ng} / \mu \mathrm{l}$ solution of WGA coupled dextran (WGA-Alexa Fluor 594, 0.5-1 $\mu$ l, Thermo Fisher) into the nasal cavities with a microloader tip. The solution was gently pipetted up and down to ensure even distribution of the 
dye without letting it spill over the cavity. Animals were kept on the moist tissue with their gills submerged in water for 10-15 min. Afterward, I washed off excess WGA-coupled dextran and let them recover in a beaker filled with tap water in the dark. After $24-48 \mathrm{~h}$, the WGA-coupled fluorophores were visible in both the MOE and the OB when checking under fluorescent illumination.

\subsubsection{Whole mount olfactory system preparation for calcium imaging}

I anesthetized tadpoles for $5 \mathrm{~min}$ in ice-chilled water until they were irresponsive. After I confirmed proper anesthesia, I sacrificed the larvae by severing the brain stem and extracted a rectangular tissue block containing the complete peripheral olfactory system and the bulb. (Schematic 14) The principal cavity was made more accessible for later odorant application, by removing excess tissue rostral of the nostril. When measuring responses in the $A O B, a$ small incision was cut into the anterior VNO to enable odorant access. The block was flipped with the ventral side up, and I carefully removed the palatial tissue covering the ventral $O B$ without injuring or stretching the ONs. The tissue block was mechanically fixed in a recording chamber via a nylon-stringed platinum grid and submerged in frog ringer.

\subsubsection{Multiple cell bulk loading (MCBL) of AM dyes into the ventrolateral MCL}

I positioned whole-mount preparations in their recording chamber under an upright stereomicroscope (Olympus BX51WI). Micro-pipettes could be mounted to a head-stage that allowed manual pressure application via an attached tubing system. Pressure could be built up via compressing air in a $50 \mathrm{ml}$ syringe connected to the tube via a three-way valve. Borosilicate-glass micropipettes with a short taper and 8-10 $\mathrm{M} \Omega$ pipette resistance were pulled for AM dye injection. I loaded the micropipettes with $3 \mu \mathrm{l}$ of the saturated calcium indicator solution. Subsequently, I checked the pipette for air bubbles or calcium indicator precipitate before mounting it and lowering it into the ringer solution. The pipette tip was penetrated into the MCL of the lateral glomerular cluster. I used a combination of the fluorescent signal of the WGA-conjugate, the cascade blue dextran added to the calcium indicator mix, and dim bright field illumination for orientation. Dye extrusion was triggered by building up pressure in the syringe and quickly opening the valve. The cascade blue dextran allowed me to estimate the extent of dye extrusion. If the injected volume was not sufficient, I either applied another pressure pulse or another injection attempt in proximity to the previous site. The calcium indicator was injected at three different locations in the MCL of the LC. The three injections covered the majority of associated MTCs but at the same time, minimizing the mechanical damage to the neuronal network. After the successful injection of the calcium indicator, the preparation was left incubating for $30 \mathrm{~min}$. Successful uptake of the AM dye was visible as a faint, but increased green fluorescent signal among the MTCs compared to the time-point of injection.

For the calcium imaging experiments in the $\mathrm{NbT}$ reporter line, I followed the same steps as described before. The only difference was that the dark red fluorescent signal of the NbT- 
positive neurons and ORN axons was used to position the micropipette for injection into the MCL properly.
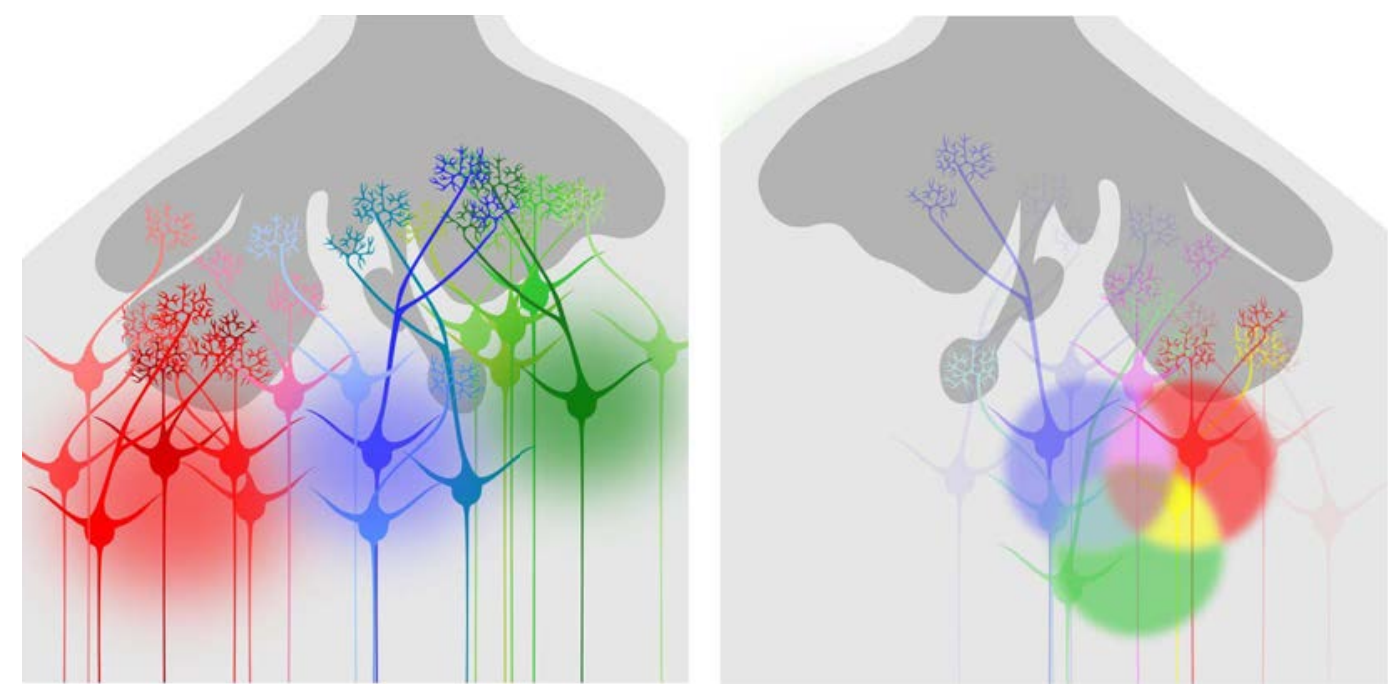

Schematic 16 Labeling of neuronal subpopulations in the $O B$ via Calcein AM dye injections

MTC subpopulations in the larval Xenopus OB can be labeled by injection of Calcein AM dyes of different colors (left panel). Injection of Calcein AM dyes at adjoining sites in the OB (right panel) can lead to labeling of MTC subpopulations and their neurites with individual color hues via uptake of different tracer ratios (see PRISMA technique)

Focal injections of AM dyes into the MCL of the ventral OB in larval Xenopus laevis offered the possibility to label subsets of MTCs, including their dendritic and axonal projections. Depending on the applied pressure, the volume and extent of AM dye extrusion could be controlled under fluorescent illumination. To anatomically label subsets of MTCs, I used Calcein AM dyes of different colors (Calcein Green AM, Calcein Orange AM, Calcein Violet AM; Thermo Fisher). By injecting differently colored Calcein AM dyes at different, but adjoining sites in the MCL, MTCs could take up different ratios of the individual dyes. These cells and their dendritic appendages thus were labeled in a different color hue than the unicolored MTCs at the injection sites. I named this method of creating concentration gradients of Calcein AM dye between three adjoining locations in space that can lead to differential dye-uptake by individual neurons' somata, PRISMA. PRISMA is an analogy to the German word for glass-prisms that can decompose 'white light' into its colors. Depending on the proximity of the injection sites and volume of injected dye, the number of labeled neurons and their diversity in color nuances can be varied.

\subsubsection{Multiphoton calcium imaging in the larval olfactory bulb}

positioned whole mount preparations loaded with Fluo-4 AM dye on the stage of the multiphoton microscope in their recording chambers. To apply odorants to the olfactory organs, I created a continuous flow with the help of a 16-channel perfusion system and a suction pump. The perfusion system (ALA-VM-8 Series; ALA Scientific) consisted of 16 syringes (50 ml) connected to a common outflow (Milli Manifold; 16 inlet ports; ALA Scientific) via silicone tubing. The gravity-fed flow of the individual channels was controlled via valve commanders (VC3-8xP Series; ALA Scientific). The different syringes were filled with around 
$20 \mathrm{ml}$ of odorant solutions and positioned around $50 \mathrm{~cm}$ above the level of the outflow. The odorant solutions used are listed in the solutions section. The flexible outflow-tube was mounted to a micromanipulator (M-MT-AB2, Newport) and lowered into the recess of the recording chamber in front of the olfactory organs. One channel was used for constant frog ringer perfusion and continuously refilled to provide a stable baseline flow. A bent syringe needle connected to a peristaltic pump (Cyclo II, Roth) via flexible silicone tubing was positioned behind the preparation. I adjusted suction speed and strength until an equilibrated flow towards the olfactory organs was created. After I lowered the objective into the solution of the recording chamber, the ventral $\mathrm{OB}$ was focused in the field of view using brightfield illumination. If the flow was stable and the region of interest visible, I occluded the setup from light and started the multiphoton imaging in one of the injected MOBs.

Before performing experiments, I probed with odorant mixtures whether there were stimulusinduced calcium responses, and the flow from the perfusion system properly reached the olfactory organ. The actual measurements in the resonant scanning mode of the multiphoton microscope, lasted 8 minutes each. I acquired image stacks of 35-40 image planes at frequencies between $0.5-0.75 \mathrm{~Hz}$. The distance between individual z-planes was in the range of 3-6 $\mu \mathrm{m}$, and the scanned volumes in the samples did not exceed $200 \mu \mathrm{m}$ in the $z-$ dimension. All recordings were done in bilinear scanning mode with $x$ - and $y$ - dimensions of $512 \times 512$ pixels. For glomerular recordings, I chose a magnification resulting in a resolution of $0,33 \mu \mathrm{m} /$ pixel whereas in the NbT-recordings I chose magnifications resulting in resolutions of $0,5-0,66 \mu \mathrm{m} / \mathrm{px}$

In the eight minutes of recordings, eight stimuli were applied to the sample. I applied each stimulus for $5 \mathrm{~s}$ after $10 \mathrm{~s}$ of baseline activity recording, before switching back to frog ringer perfusion. One channel was filled with $20 \mathrm{ml}$ of frog ringer as a control (ringer control). This was to ensure that observed responses were not due to the pressure differences arising from switching between the continuous frog ringer perfusion and the odorant solution channels. After the $5 \mathrm{~s}$ stimulation interval, the sample was recorded for another $60 \mathrm{~s}$ before applying the next stimulus. Complete datasets of MTC activity typically included two recordings with the stimulus sequence 1 (basic/aromatic mix, H, R, K, F, W, ringer control, basic/aromatic mix) and two recordings with the stimulus sequence 2 (short-neutral-chain mix, long-neutralchain mix, M, L, I, V, alcohol/aldehyde/ketone mix, long-chain-neutral mix) to repeat every stimulus at least twice and the amino acid mixes at least twice per recording. Despite mechanical fixation via the platinum grid slight drift of the sample along all three dimensions was unavoidable in the 32 min overall recording time. This drift was more impactful at the higher magnifications in glomerular activity measurements. While I performed four stimulus sequences per experiment, a complete dataset of glomerular activity for analysis thus consisted only of the two different stimulus sequences without a second repetition. 


\subsubsection{Processing and evaluation of calcium imaging data}

I mainly processed and evaluated the calcium imaging experiments using the programming language Python on the Jupyter-notebook interface (jupyter.org). The code for calcium imaging analysis used for this work is composed of different modules. Individual modules can differ in the origin of source code and contributions in code writing. The key to the individual modules' origin and contributions is documented in the code and accessible upon request from the authors Thomas Offner or Dr. Thomas Hassenklöver (t.offner@gmx.de; thomas.hassenkloever@physzool.bio.uni-giessen.de).

Generally speaking, the volumetric time series underwent a sequence of processing steps that I will sketch in the following paragraph. First, the raw image files and their metadata were transformed into a format that made them processable in Python. Artifacts from bilinear resonant scanning mode were removed, and the individual measurements of eight stimuli concatenated into a single 4-dimensional array. To compensate for the drift of the sample in the z-dimension, I combined groups of 6 subsequent image planes to one plane by creating maximum intensity z-projection from them. To obtain 'drift-free' image planes, I ran a piecewise-rigid motion correction algorithm (NoRMCorre; (Pnevmatikakis and Giovannucci, 2017) The resulting pre-processed calcium imaging data were used for further steps like denoising, deconvolution, and de-mixing by the calcium imaging analysis toolkit CalmAn (Giovannucci et al., 2019) based on algorithms described in detail in (Friedrich et al., 2017; Pnevmatikakis et al., 2014, 2017).

After denoising, the data, constrained non-negative matrix factorization was performed to extract the spatial and temporal components of the measurements (Pnevmatikakis et al., 2014). The parameters I used to detect odorant responsive regions of interest (ROIs), and their fluorescence intensity time-course in CalmAn are documented in the code used for this thesis. As side-products of the CalmAn pipeline and the CNMF algorithm, movies of the denoised data could be created. Another useful image series created in the process only displayed the spatial components and their deconvolved fluorescent time-courses that fulfilled the criteria set beforehand. The resulting 'only neurons' movie (Giovannucci et al., 2019) only included the denoised and deconvolved fluorescence intensity changes of odor responsive ROIs that classified as neurons/glomeruli.

One way to visualize odor response profiles of stimulus-responsive ROIs was to create fluorescence intensity difference maps from those movies. Intensity difference maps were created by subtracting the peak fluorescence intensity (mean of time-frame $3 \mathrm{~s}$ post-stimulus $+/$ - 1 frame) of stimulus correlated responses from baseline fluorescence prior to the stimulus (mean of time-frame $5 \mathrm{~s}$ prior to stimulus +/- 2 frames). I created the glomerular odor maps in this work by using the 'only-neurons' movie as a template for intensity difference map generation. I merged the intensity difference map image stacks of individual stimuli to multicolor pictures in Fiji (Schindelin et al., 2012). 
Each deconvolved fluorescence intensity time-trace was normalized by the maximum stimulus correlated response amplitude of the entire time-series (10 s interval after stimulus application). Responsive ROIs (spatial components plus their fluorescence intensity timecourse) underwent several steps of filtering and quality control. First, I only used responsive ROIs that had unambiguous odor responses to at least one of the odor mixes (amplitudes exceeding the median of the entire time trace's fluorescence intensity values). Second, I only used ROIs with positive responses in all repetitions of the individual stimuli. Third, I only used ROIs with fluorescent time traces in which the mean fluorescence intensity of all pre-stimulus intervals (10 s intervals, 20 s before stimulation), exceed the standard deviation value of each trace's fluorescence intensity distribution. Selected responsive ROIs were manually categorized into JGCs or glomeruli (based on the WGA-Alexa Fluor 594 staining) or into NbTpositive/negative neurons (based on presence of cytosolic fluorescence of the red fluorescent protein Katushka).

\subsubsection{Analysis and visualization reactive regions to sulfated steroid responses in the $M O B$ and $A O B$}

All scripts for the visualization and analysis of reactive regions in the $M O B$ and $A O B$ to sulfated steroids were written in MATLAB (Mathworks, Natick, USA) by Thomas Hassenklöver. Figure 22 is published in (Sansone et al., 2015) and was created by Thomas Hassenklöver based on my calcium imaging data. Difference image stacks were calculated via subtraction of the pre-stimulus baseline activity from the mean peak response of reactive regions. The difference image stacks underwent plane-wise, adaptive thresholding to convert them to binary image stacks. Those binary data were smoothened and either used for 3D reconstruction of reactive regions or as mask to extract single fluorescence time traces of glomeruli or MTCs.

\subsubsection{Processing and evaluation of tracings with WGA-coupled fluorophores}

Multi-channel 'lambda stacks' were acquired with the spectral fingerprinting function of the 32-channel detector of the LSM780 confocal microscope. For spectral matching between voxels of the acquired 32-channel, 3D image stacks, I performed the voxel-wise division of each channel's fluorescence intensity value by the summed intensity values of all 32 channels. The similarity between one reference voxel of the GL in the OB and the voxels of the imagestacks, including the olfactory organs were calculated by the element-wise subtraction of the two spectral fingerprints and calculation of the mean of all 32 differences' absolute values. This value of spectral similarity between voxels (ranging between 0 and 1 ) was also used to create 16-bit images for visualization of matching pixels' distribution. I wrote the scripts for this analysis in Python. 


\subsubsection{Result visualization}

I visualized my results with graphs created in Python using the Seaborn (version 0.9; 10.5281/zenodo.1313201), matplotlib (Hunter, 2007) and NetworkX (NetworkX developer team (2014)) packages or the open-source image processing software Fiji (Schindelin et al., 2012). MTC morphologies from sparse-cell electroporation experiments were reconstructed using the 3D image stack as a reference and draw dendritic trajectories in the vector graphic program Inkscape. I drew all schematics in introduction, methods and discussion with Inkscape myself (Harrington, et al., (2004-2005); Inkscape. http://www.inkscape.org/).

\section{RESULTS}

\subsection{A VENTRO-LATERALLY LOCATED ORN POPULATION INNERVATES THE LATERAL GLOMERULAR CLUSTER IN LARVAL XENOPUS}

I injected four differently colored WGA-coupled fluorophores (Alexa Fluor 488, 555, 594, and 647 ) into four defined regions of the larval OB. I did this to predict ORNs' axonal target region in the OB by the spectral fingerprint of the vesicular structures in their somata (Tsuriel et al., 2015). I scanned the entire sliced MOE of the larvae $24 \mathrm{~h}$ after injection using spectral emission fingerprinting in the lambda-scanning mode of the confocal microscope. The injection of multiple fluorophore-coupled WGA conjugates led to punctuate glomerular labeling in the MOB and the $\mathrm{AOB}$. The injections nicely stained groups of glomeruli.

In contrast to glomerular labeling via bulk electroporation, there were no axonal fiber components stained (Figure 1, B). I recorded ten OBs in larval stages between 51 and 54. In all of them, it was possible to distinguish two adjacent but distinguishable 'lobes' in the LC. I will henceforth name them ventromedial lobe and dorsolateral lobe (Figure 1, B). The emerging dorsal OB was visible on the medial side, dorsally of the MC. Gradual, overlapping distributions of the different WGA-coupled fluorophores were apparent, especially in the ORN part of the GL. I observed anterograde and retrograde transport of tracer-filled vesicles in the whole-mount preparation (not shown). Already $24 \mathrm{~h}$ after injection, massive amounts of tracer-filled vesicular structures were visible in the olfactory organs. Due to a lack of counterstaining, it was not possible to analyze the 'basal to apical' distribution of the tracerfilled vesicular structures. Strong punctuate labeling of the ON, and more than $1000 \mu \mathrm{m}$ of distance between the olfactory organs and the $\mathrm{OB}$, suggested active retrograde transport to ORN somata. Spectral fingerprints of vesicular structures in the MOE and VNO were diverse. Even taking only 3 of the recorded 32 emission channels into account, the spectral fingerprints of the vesicles and their distribution among the olfactory surfaces nicely reflected already described axonal connectivity in larval Xenopus (Gliem et al., 2013; Hassenklöver and Manzini, 2013). Spectral fingerprints observed in the VNO matched with the AOB and vesicular structures with tracer compositions as seen in the LC were found spatially clustered 
in the lateral part of the MOE (Figure 1, B and C). These findings implied that necessary conditions for the Neuronal Positioning System with WGA tracers by Tsuriel and colleagues (Tsuriel et al., 2015) were met in Xenopus laevis. Consequently, I chose spectral profiles of individual regions of interest among the glomerular clusters (for example of a pixel in the ventromedial lobe of the LC; black cross Figure 1, D) and calculated the spectral similarity of every pixel of the image stack to this region's spectral profile (visualized as intensity value; see methods section for details). Those similarity heatmaps were generated for the OB image stack (Figure 1, D) and the image stacks of the corresponding olfactory organs (Figure 1, E and F). I could observe clear lateralization of vesicular structures matching the LC's spectral fingerprint in the lateral MOE, more precisely in the 'middle sections' of the whole olfactory organ (yellow puncta, Figure 1, E). In the most dorsal slice of the MOE, this clear lateralization was not apparent, and signal matching the LC was either sparsely distributed or non-existent (not shown). High numbers of vesicular structures spectrally matching to the LC were present in ventral sections of the MOE around the choane, without bias to either the medial or lateral side (Figure 1, F). This ventrolateral distribution of ORNs innervating the LC was observed in multiple animals. Individual slices were scanned from both sides to exclude tissue depthdependent limitations in the detection of tracer fluorescence. Vesicular structures matching the MC's spectral fingerprint (Figure 1, D) were found broadly dispersed throughout the MOE with no obvious spatial clustering along the mediolateral or the ventrodorsal axes (white cross, Figure 1, D, E and F).

\subsection{MTCS SHOW TOPOGRAPHICAL ASSOCIATION WITH THEIR NEIGHBORING GLOMERULAR CLUSTER WHICH IS ALSO MIRRORED IN THEIR DENDRITIC AND}

\section{AXONAL PROJECTIONS}

To investigate, whether the anatomical segregation of the lateral and the medial olfactory streams observed by (Gliem et al., 2013) is conserved on the level of the MTCs in larval Xenopus laevis, I labeled different subsets of MTCs via multi-color Calcein AM dye injections (Figure 2, B). I chose injection sites among the MCL, which were in proximity to the LC, intermediate cluster, medial cluster, or AOB. It was possible to label big groups of MTCs and other neurons but still control the extent of individual dye injection along the mediolateral axis (Figure 2, C). Somata, dendritic structures, and axons could already be visualized by this technique after 30 min of incubation time. It was not possible to selectively label MTCs. However, I could distinguish their characteristic tufted dendrites as main contributors to the postsynaptic glomerular neuropil (See also Figure 6, A). The Calcein AM dye injection experiments revealed that MTCs' somatic proximity to a defined glomerular cluster was predictive of their primary dendritic contributions to this cluster's glomerular neuropil. The MTC population seemed to be coarsely topographically arranged in the larval OB (Figure 2, C). 

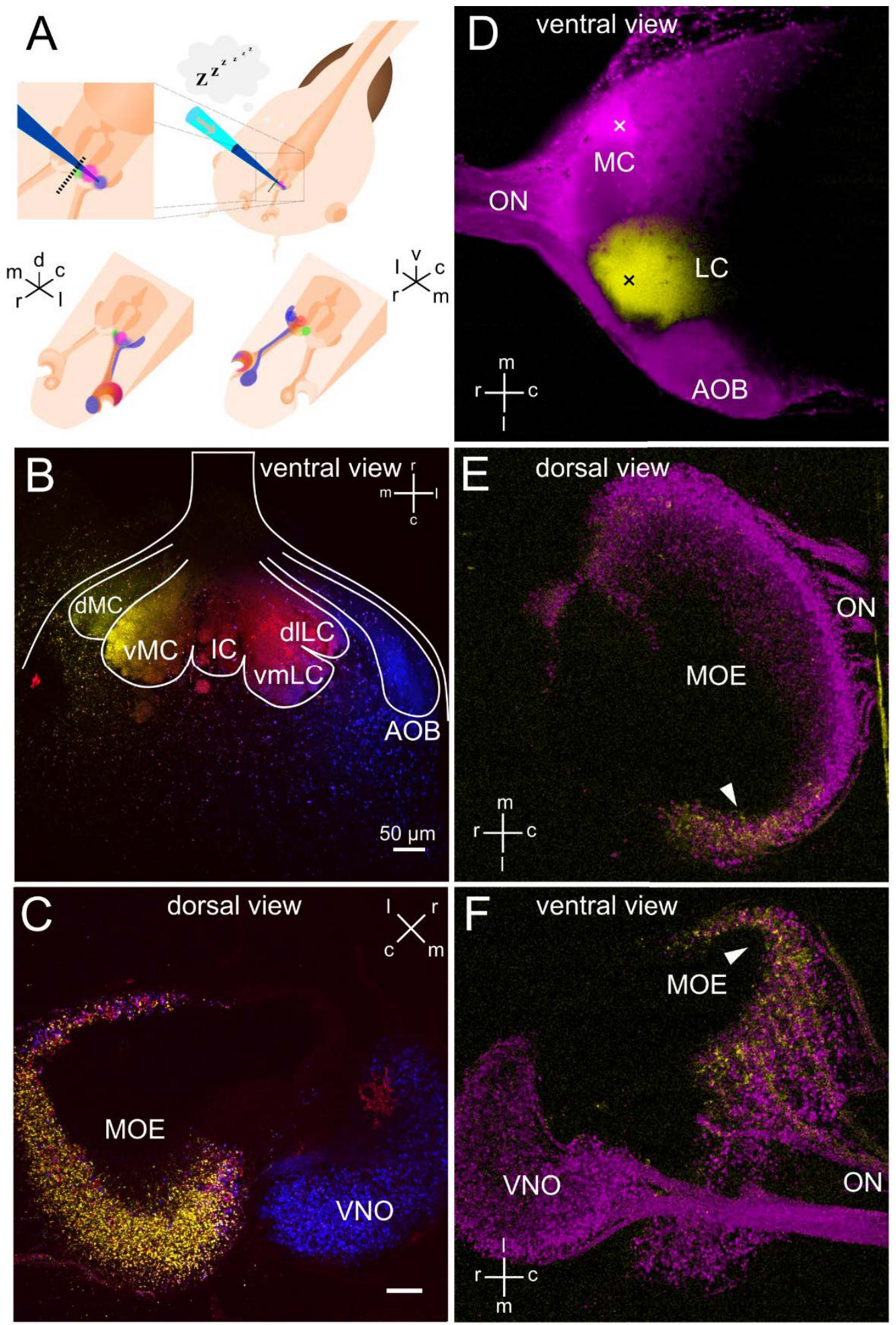

Figure 1 Ventrolateral distribution of ORNs innervating the lateral glomerular cluster revealed by multicolor retrograde tracing with WGA-coupled fluorophores

A) Schematic of multi-color dye injection into the OB of Xenopus laevis tadpoles. Dye spread in the olfactory explant after $24 \mathrm{~h}$ is illustrated from the dorsal and ventral view B) WGA-coupled Alexa Fluor dye distribution in the ventral larval Xenopus OB, imaged in tissue sections $24 \mathrm{~h}$ after injection. Different WGA-coupled Alexa Fluor dyes are labeled in false colors. C) Distribution of retrogradely transported WGA-coupled dyes in the olfactory organs from the respective OB (B) of the injected animal. D, E and F) Tracing of the ORN populations innervating the LC via spectral fingerprinting of tracer molecules D) Intensity heat map depicting spectral similarity of all pixels to one lateral (yellow; black cross) and one medial (magenta; white cross) region of interest. E) Lateralized distribution of vesicular structures matching the LC's spectral fingerprint (yellow puncta) in a dorsomedial section of the corresponding larval olfactory organs. F) Ventral view on vesicular structures matching the LC's spectral fingerprint in the ventral MOE. The signal (yellow) was found enriched around the choane, with no apparent bias on the mediolateral axis 
I could not observe dendritic projections from neurons of the lateral OB to the glomerular array of the medial OB and vice versa. However, individual dendritic branches of MTCs that projected to an adjacent glomerular cluster occurred (Figure 2, C; asterisks). Primary dendritic projections from MTCs of the AOB to the MOB glomerular array and vice versa were not present in the animals and developmental stages I imaged.

The whole-mount preparation could be kept alive for at least 1.5 to $2 \mathrm{~h}$ in frog ringer. The additional time allowed the AM dyes to travel into the thin axons of MTCs and made them possible to image at higher laser powers. Due to the presumably diffusion-limited distribution of Calcein dyes, the extent of axonal labeling could not always be easily estimated. However, it was often possible to follow axonal trajectories for several hundred micrometers towards the telencephalon or even farther higher brain centers (Figure 3, A). The coarse topographical arrangement of MTC populations along the mediolateral axis was also conserved in the Calcein labeled axonal bundles: A considerable amount of fibers from the medial or lateral OB converged to the loosely arranged medial or lateral olfactory tract, respectively (Figure 3, $A, B, C$ and D). In addition to the two main tracts, sparse fiber bundles projected in a fan-like, coarsely topographical manner across the entire surface of the $\mathrm{OB}$, ventrally of the ventricle.

\subsection{Projections OF MTCS' APICAL DENDRITES ARE ONLY COARSELY TOPOGRAPHICALLY ARRANGED WITHIN THEIR ASSOCIATED GLOMERULAR CLUSTER}

After I observed that MTCs were topographically associated with their neighboring glomerular clusters, I wanted to find out whether this topographical association exists also on the smaller level of individual glomerular clusters. By injecting differently colored Calcein AM dyes at three adjoining sites in the MCL of the LC, I labeled small subsets of neurons. The individual colors of their somata and dendritic projections to the GL resulted from the different ratios of colored AM-dyes taken up by the neuronal cell bodies (see methods section; PRISMA). Medially located subset of MTCs (and other labeled OB neurons in that layer) projected their dendritic contributions to the neuropil to more medial glomeruli (Figure 3, E and H). The dendritic projections of laterally located neurons had a bias to the more lateral parts of the glomerular cluster (Figure 3, E and F). Neurons located in between those two populations projected their dendrites to more central parts of the LC (Figure 3, E and G). This coarsely topographic clustering of neurons was also observed along the dorsoventral axis to a certain extent. However, the dendritic projections of ventral subsets of MTCs were often intermingled (Figure 3, I, J, K and L). Dendrites of more laterally or medially located MTCs could be observed in the contra-lateral parts of the glomerular array (asterisks, Figure $3, \mathrm{~F}$ and $\mathrm{H}$ ). Overall there seemed to be a crude bias of MTCs to project their tufted dendrites to proximal glomeruli of the LC. This bias was less pronounced however than on the macroscopic scale. 

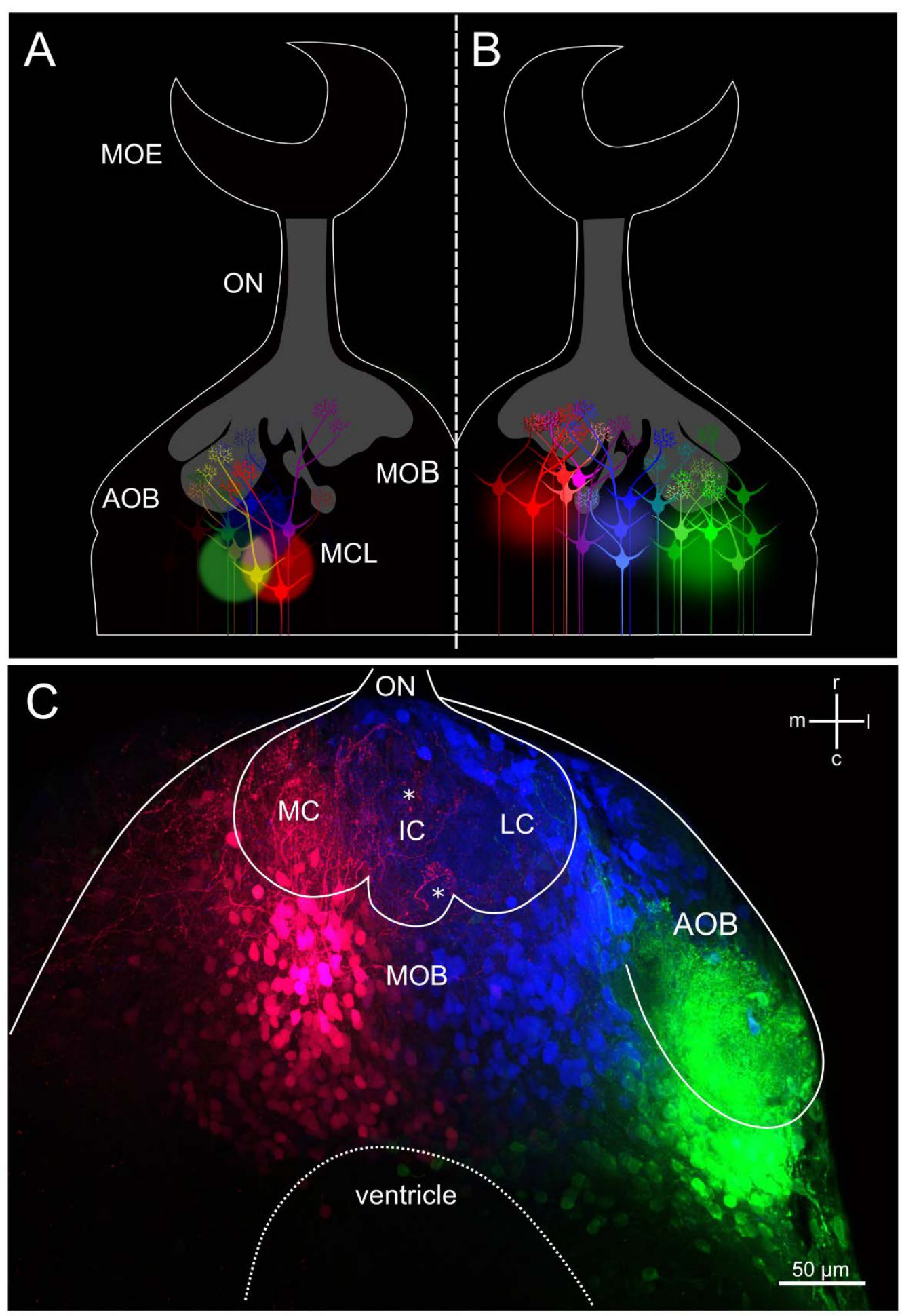

Figure 2 Labeling of MTC populations and their dendritic projections associated to the glomerular clusters

A) Schematic of Calcein AM dye injections at proximal adjoining sites in the ventral olfactory bulb of the whole mount preparation (PRISMA technique). B) Schematic of Calcein AM dye injection at three sites among the MCL to label neuronal cell bodies (MTCs) and primary dendritic projections to the glomerular clusters of the MOB or the AOB. C) Representative example of topographical association of MTCs and other neurons of the MCL to their neighboring glomerular cluster. Individual primary dendrites from the medial neuronal population (red) were observed to project to the intermediate cluster (IC) with their primary dendritic tufts (asterisks). MTCs of the larval $A O B$ did not project tufted dendrites to the MOB and vice versa. 


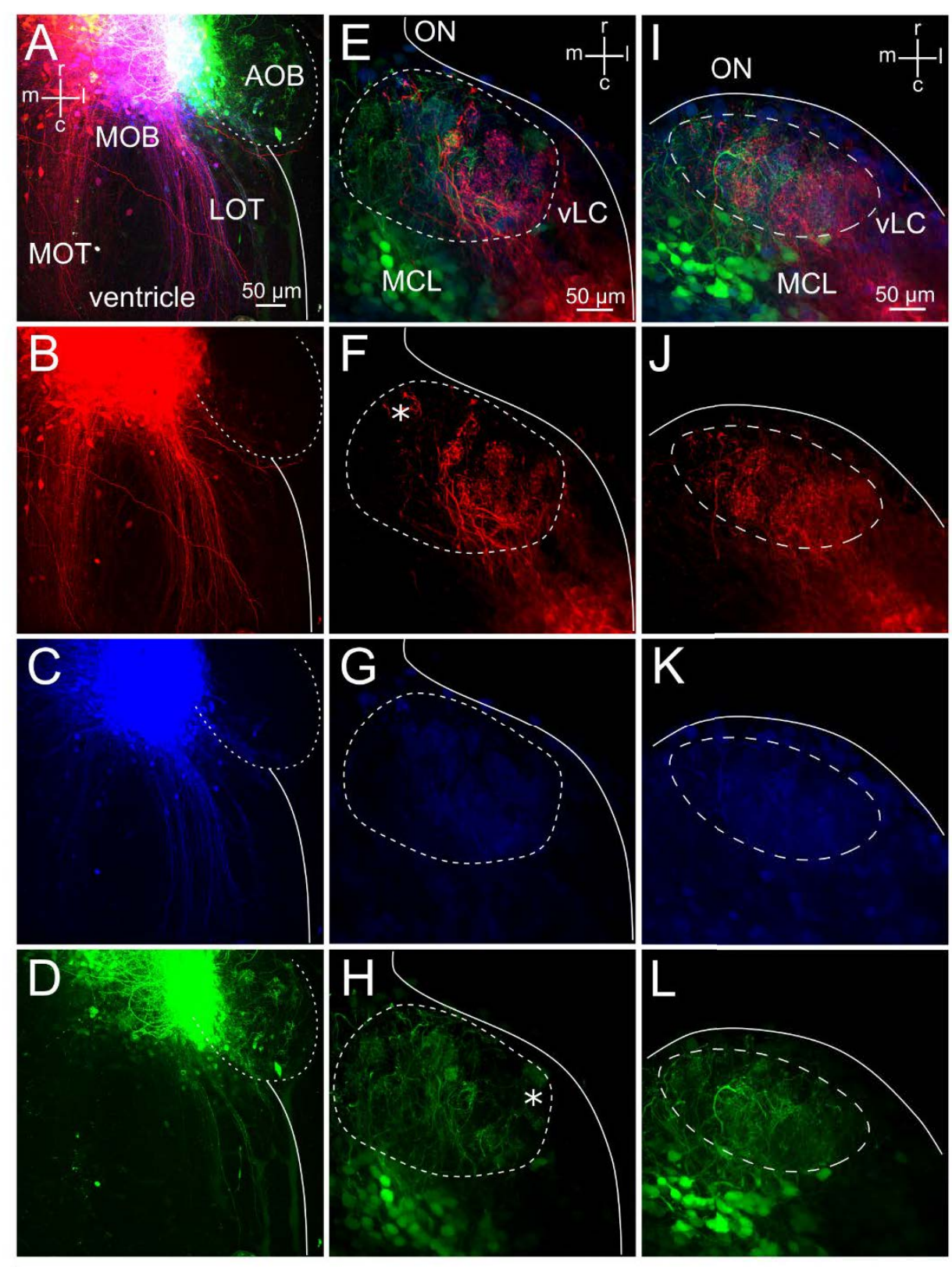

Figure 3 Coarse topographic arrangement of MTCs' axonal projections and apical dendrites

A, B, C and D) Axonal projections of different MTC subsets along the mediolateral axis. Different colors represent different Calcein AM dye injections in the $\mathrm{OB}$ (B, C and D). Axonal bundles group into the coarsely organized lateral and medial olfactory tract (LOT, MOT). Axons project caudally in a fan-like manner on the OB surface ventrally of the lateral ventricle. E, F, G, and H) Coarsely topographic projection areas of postsynaptic neurons' dendrites to the GL. Lateral neuronal populations project tufted dendrites to the more lateral glomeruli $(F)$ mediallybiased populations project their dendrites to more medial glomeruli $(\mathrm{H})$. Individual tufted dendrites are present in the contralateral part of the GL (white asterisks). I, J, K, and L) Dendritic contributions of different neuronal populations in a more ventral level of the same animal's GL. Dendritic projections in the ventral part appeared more intermingled in this animal. 


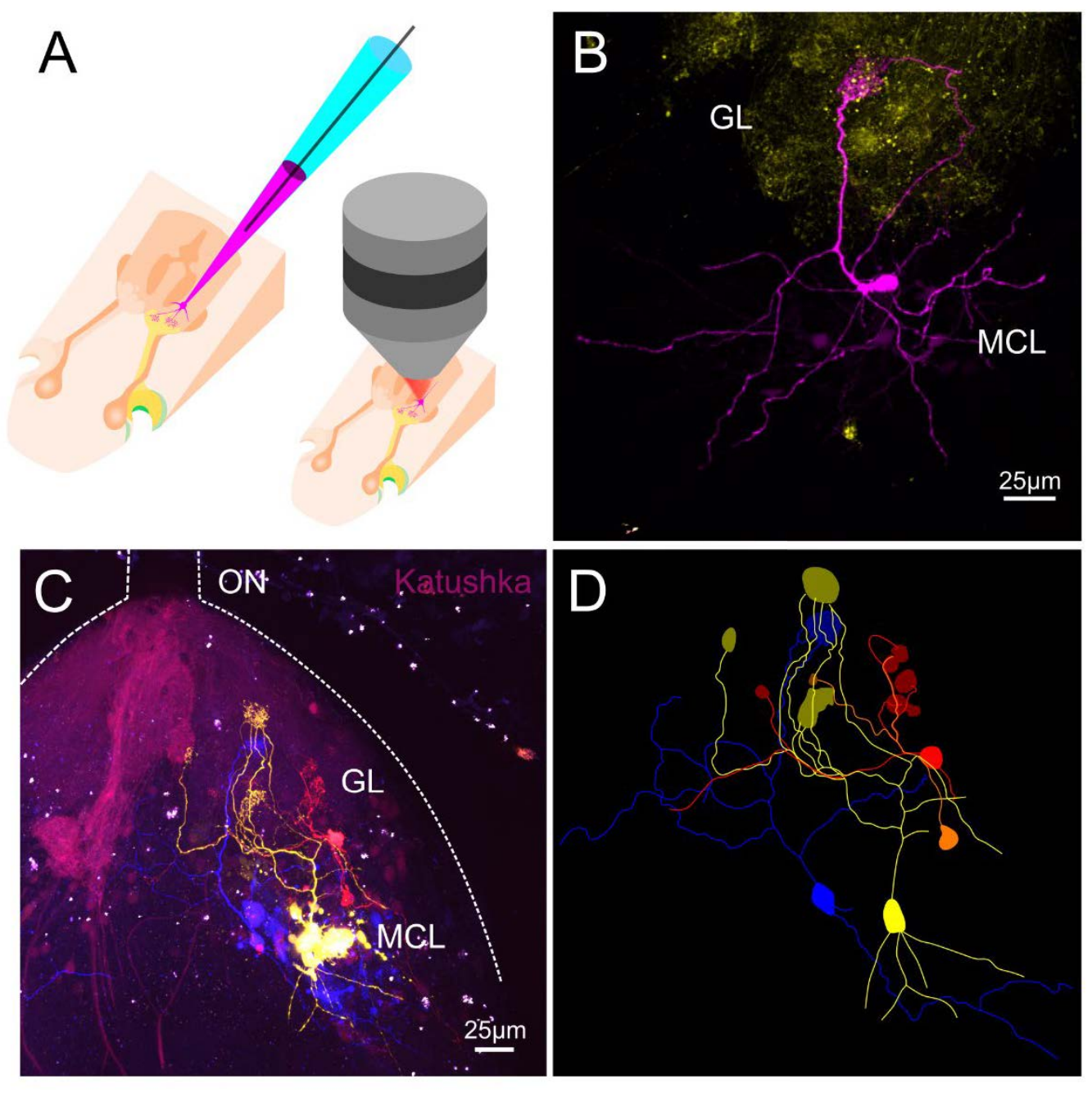

Figure 4 Morphological staining of MTCs via sparse-cell electroporation in the MCL of larval Xenopus laevis

A) Schematic representation of the sparse-cell electroporation method in the whole-mount preparation of the larval olfactory system. Single MTCs in the MCL (magenta) are electroporated with dextran-coupled fluorophores. ORNs labeled with a dextran-coupled fluorophore of different color (f.ex., bulk electroporation) serve to navigate the pipette to the MCL (yellow). MTC morphologies were imaged in the whole-mount preparation using multiphoton microscopy. B) Example of single MTC (magenta). Glomeruli are stained by ORN electroporation with Oregon Green 488 BAPTA dextran (yellow). C) Multi-color dextran electroporation in the NbT-Katushka reporter line. NbT-expressing MTCs and ORN axons are visible by Katushka fluorescence (magenta). Individual MTCs electroporated with dextran-coupled fluorophores (blue, yellow and red). D) reconstruction of MTC main morphological features (tufts, dendrites and soma) in a 2D maximum projection.

\subsection{MTCS ARE VERY HETEROGENEOUS IN THEIR INDIVIDUAL DENDRITIC CONFIGURATIONS BUT CAN BE CLASSIFIED BY NUMBER OF TUFTED \\ PRIMARY DENDRITES}

I established the sparse cell electroporation protocol in the MCL of larval Xenopus OB to have a high-throughput method for gathering 'authentic' morphological data of individual MTCs in the intact olfactory system explant (Figure 4). I electroporated and reconstructed MTCs of wildtype (Figure 4, B) and NbT-Katushka animals (Figure 4, C and D) to categorize existing morphological subtypes and their frequency among the MTC population. I electroporated and reconstructed the morphological features of 77 MTCs from 35 OBs of 29 wildtype and 18 OBs of 9 NbT-Katushka animals. MTCs were very heterogeneous in their 
morphology. I categorized them into three subgroups based on their tufted primary dendrites: 40,2 \% (31 MTCs) formed one cohesive tuft with their apical dendrites, 42,9 \% (33 MTCs) bore two anatomically distinct tufted dendrites and 11,7 \% (9 MTCs) even exhibited three or more tufts on their primary dendritic branches. Four cells $(5,3 \%)$ did not show any apparent tufts on their extensive primary dendritic arbors at all (Figure 5, lower part). Virtually all MTCs possessed a common primary dendritic trunk that branched into primary tufted or secondary dendrites. Since the axons were not distinguishable from basal dendrites with the method, I used (see discussion), both were treated as 'basal neurites' (basal neurites: grey, Figure 5). In MTCs bearing one or two tufts, individual or multiple secondary dendrites could emerge from virtually every level of the primary dendritic tree. They also varied remarkably in length (in the range of few dozens to more than $150 \mu \mathrm{m}$ ). The sub-classification of MTC types, according to secondary morphological features, aims to highlight common dendritic configurations among groups of MTCs rather than to distinguish between different functional cell-types. MTCs with one tuft could possess both, multiple secondary dendrites and numerous basal neurites (Figure 5; type A I). Other subtypes exhibited either more (Figure 5; type A II) or fewer (Figure 5; type A III) secondary dendrites compared to the number of basal neurites. A small group seemingly possessed no secondary dendrites at all (Figure 5; type A IV). In some MTCs only one secondary dendrite and few (Figure 5; type A II) to no (Figure 5; type A V) basal neurites were found. In a subset of MTCs, the tuft consisted of multiple primary dendrites, of which at least one had loop-like trajectories before converging to the single tuft (Figure 5; type A III).

Different morphological subtypes were found among the MTCs with two distinct tufted dendrites. MTCs could possess higher (Figure 5, type B II, III, IV) or comparable (Figure 5, type $\mathrm{B} \mathrm{VI}$ ) numbers of secondary dendrites compared to the basal neurites. Long secondary (occasionally bifurcating) dendrites emerged either from the shared primary dendritic trunk (Figure 5, type B II), the primary tufted dendrites (Figure 5, type B IV) or from both (Figure 5, type B III). MTCs with either multiple (Figure 5, type B I, B VI), or few to no (Figure 5, type B III) basal neurites (Figure 5, type B II, IV, V) were present. The diameter of most of those tufts was below $25 \mu \mathrm{m}$ except a group of MTCs, that exhibited non-spheroidal tufted arborizations. Those tufts were often interconnected by an extensive network of primary dendritic branches (Figure 5, type B VI).

MTCs that bore more than two tufts were rarer and less diverse in their general dendritic configurations. In type C I, individual tufted dendrites branched off from a common primary dendrite (Figure 5, type C I). In other cells, individual tufts consisted of multiple tufted dendrites that branched off at several levels of the primary dendritic stem (Figure 5, type C. II) I also found one MTC in which from every one of its tufts small, blunt-ended dendritic protrusions emerged. On one of those protrusions, a small arborization was visible, but it was arguable whether it was a proper fourth tuft (Figure 5, type C. III). 


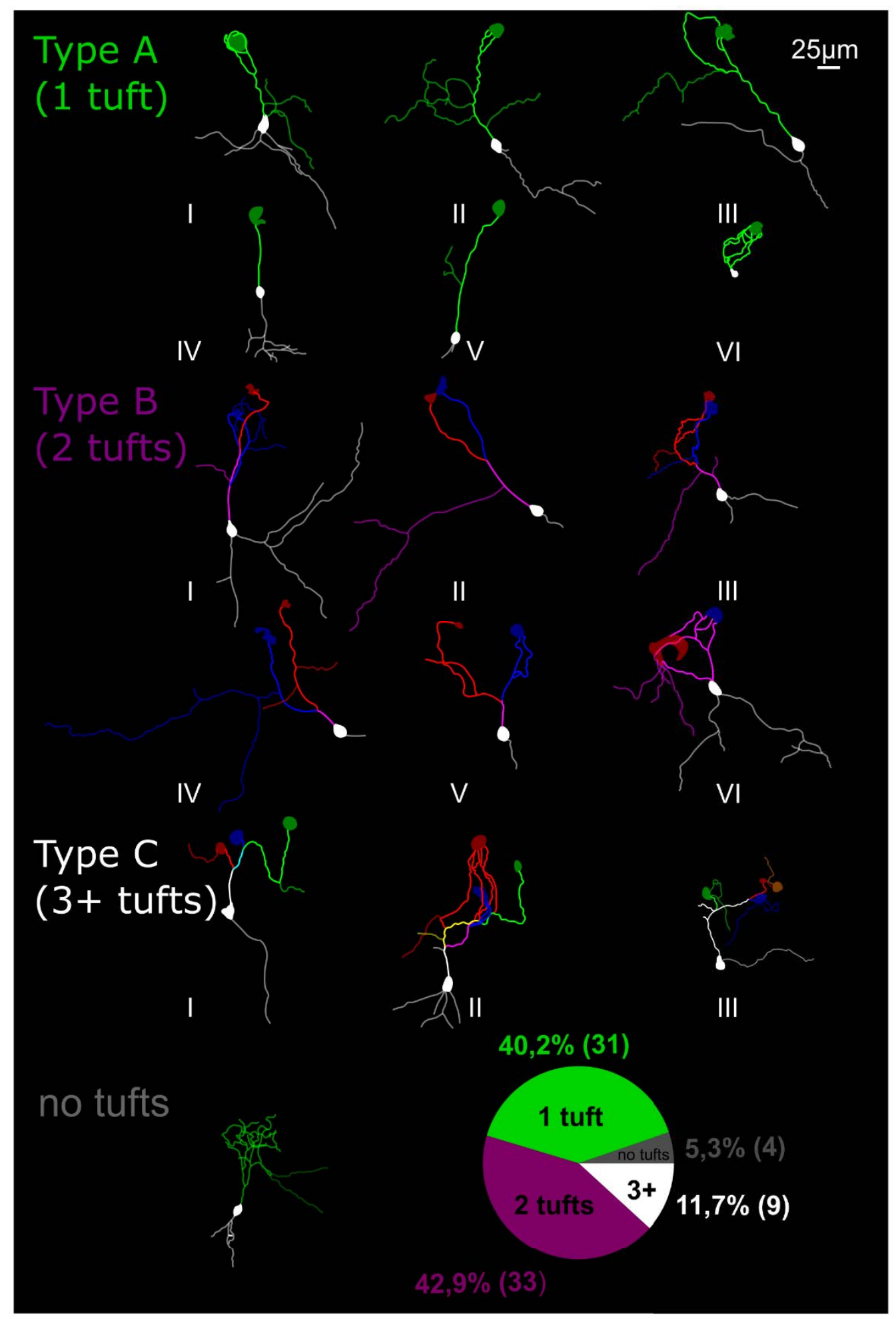

Figure 5 MTC morphological subtypes regarding number of tufted primary dendrites and neurite distributions

MTC morphologies can be subdivided by number of anatomically distinct tufts. (Type A, B and C). Type A MTC primary dendrites (light green) innervate single tufts (green areas). Type A MTCs can be further divided into subtypes (I-VI) by the different numbers and ratios of secondary dendrites (darker green) and basal neurites (grey). Type B MTCs possess two primary apical dendrites (light red and light blue) bearing anatomically distinct tufts (blue and red areas). Type B MTCs can further be subdivided by the number and ratio of basal neurites (grey) and secondary dendrites. Subclassification was additionally based on secondary dendrites' emergence from the shared primary dendritic trunk (magenta; dark magenta) individual tufted dendrites (dark blue or dark red) or from both. Type C MTCs bear more than two tufts and were subdivided into three types (I-III) based on number of primary dendrites (light blue, green and red dendrites) that innervate the tufts (blue, green and red areas) and the existence of blunt (dark green and blue) or tufted dendrites emerging from primary tufts (orange). MTCs with no tufts classified as an independent type. The piie-chart diagram represents ratios and numbers of the different MTC types (A-C and no tufts) 


\subsection{MTCS WITH MULTIPLE TUFTS CAN INNERVATE MULTIPLE ANATOMICALLY DISTINCT GLOMERULI}

Due to the unparceled character of the lateral glomerular cluster, it was not always easy to tell whether MTCs that bear multiple distinct tufts also innervate multiple glomeruli. I managed to electroporate sister-MTCs (2 pairs) that both innervated two anatomically distinct glomeruli (Figure 6, E). The individual tufts shared the glomerular volumes, and several primary dendrites of both cells contributed to both glomeruli. The PRISMA technique, which I established to differentially color MTCs and their dendritic projections, helped to estimate the glomerular volumes even in the unparceled parts of the glomerular array (Figure 6, A). Glomerular boundaries were distinguishable by differently colored dendritic contributions. The differently colored dendritic tufts were tightly intermingled. In some glomeruli, only dendrites of certain colors were observable (Figure 6, B, C and D; numbered, encircled glomeruli). The differently colored, dendritic arborizations in the individual glomerular territories, were very similar in size. The glomerular sizes were in the range of the tuft sizes observed in electroporated MTCs ( $25 \mu \mathrm{m}$ or smaller in diameter). These observations do not rule out the existence of bigger glomeruli. They rather highlight the tight 'packing' of glomeruli innervated by different subsets of MTCs on the glomerular array. 

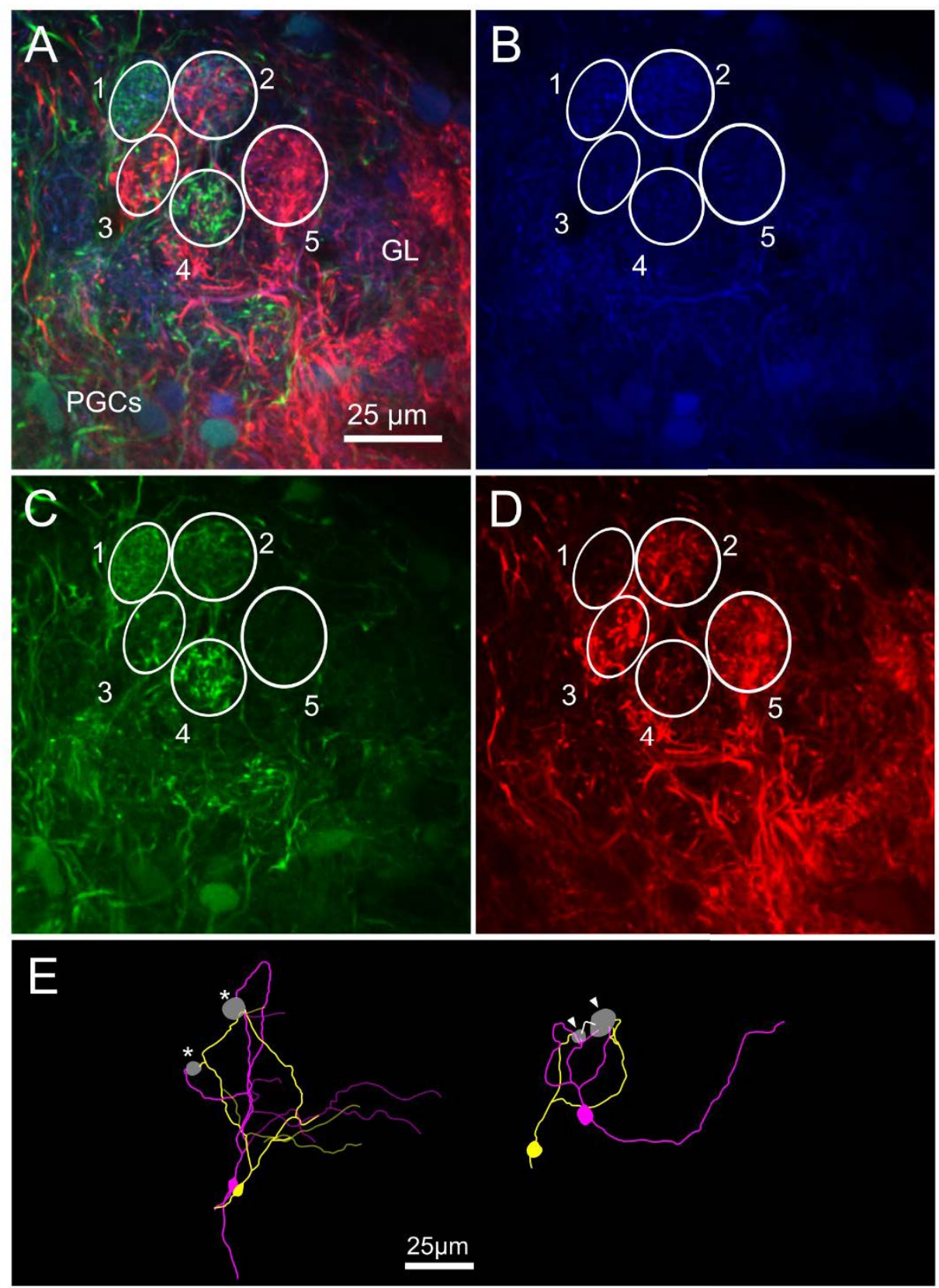

Figure 6 Dendritic contributions to glomerular volumes and multi-glomerular sister-MTCs

(A-D) Differential labeling of subsets of neurons in the LC and their tufted dendritic contributions to glomerular volumes (white outlines, numbers 1-5). Individual glomeruli were composed of dendrites from different neuronal populations (f.ex glomeruli 1, 2 and 5; B, C and D). E) Pairs of reconstructed sister-MTCs (yellow and magenta) innervating the same two, anatomically distinct glomeruli (grey areas; white asterisks or arrowheads).

\subsection{NBT PROMOTOR ACTIVITY LABELS A SUBSET OF MTCS AND ORNS IN LARVAL Xenopus OB}

After I could characterize MTC morphologies, their glomerular innervation patterns and distribution on the population level, I searched for possible markers that might selectively label MTCs in our model organism. After a dozen antibodies I tried, I found a promising, yet poorly investigated, transgenic line from the European Xenopus Resource Center. I performed multiphoton imaging of the $\mathrm{OB}$ of transgenic tadpoles expressing the deep red fluorescent protein Katushka under the Xenopus NbT promotor. Katushka fluorescence was 
present in ORN somata and their axons (Figure 7; A and B). Moreover, several ellipsoid somata with a small apical dendritic trunk were observable on the level of the MCL (Figure 7, $B$; white crosses). In the GCL, there seemed to be no signal at all. To validate that the $\mathrm{NbT}$ positive neurons were MTCS, I performed sparse-cell electroporation, targeting the fluorescent somata of NbT-positive cells in the MCL (See also Figure 4, C). However, due to the dense, surrounding dendritic meshwork from other neurons, additional cells than the one targeted were labeled by the green and orange dextrans I used. The spectral bleed-through of those dyes into the deep red emission channel of the multiphoton microscope was so high that the initial Katushka fluorescence could not be unambiguously confirmed afterward. Nevertheless, it can be stated that in none of the experiments I tried targeting NbT-positive cells, any other cell type but MTCs was labeled. The NbT-positive cells were not the only neurons with MTC morphologies. I managed to electroporate a small number of NbT-negative neurons in the MCL with a blue fluorophore-coupled dextran that had no bleed-through into the red channel and displayed MTC morphology (Figure 4, C and D; blue neuron) ). Given the limited number of animals I had for these experiments, I was not able to compare the morphologies of NbT-positive and NbT-negative neurons.

\subsection{SubSets of NBT POSITIVE MTCS SHOW RESPONSES to AMINO ACID ODORANTS}

The activity of the NbT promotor driving Katushka expression in the transgenic line I used has been associated with neurogenesis and axogenesis in Xenopus embryos (Moody et al., 1996). Therefore, I performed calcium imaging experiments in the whole-mount preparation with amino acids as odor stimuli to test whether NbT-positive MTCs receive synaptic input from ORNs. The calcium imaging experiments in the transgenic NbT reporter line revealed that a part of the NbT-positive MTC population was responsive to single amino acids as (Figure 7; white empty arrowheads). However, a substantial portion of the NbT-positive neurons did not respond to the odorants (Figure 7; white asterisks). Since the CalmAn algorithm excluded spatial components that did not respond to odor stimuli, the exact ratio between responsive and non-responsive NbT-positive cells was not possible to quantify. Odorant responses were also observed in neurons of the MCL that did not show evident Katushka fluorescence (Figure 7; white arrowheads). It seemed that the $\mathrm{MCL}$ in larval Xenopus was composed of NbT-positive and NbT-negative MTC populations, each of them, to a certain extent responsive to amino acids as odorants. The detailed odor tuning of MTCs will be described in the following sections that cover the odor information processing at the different levels of the OB. 

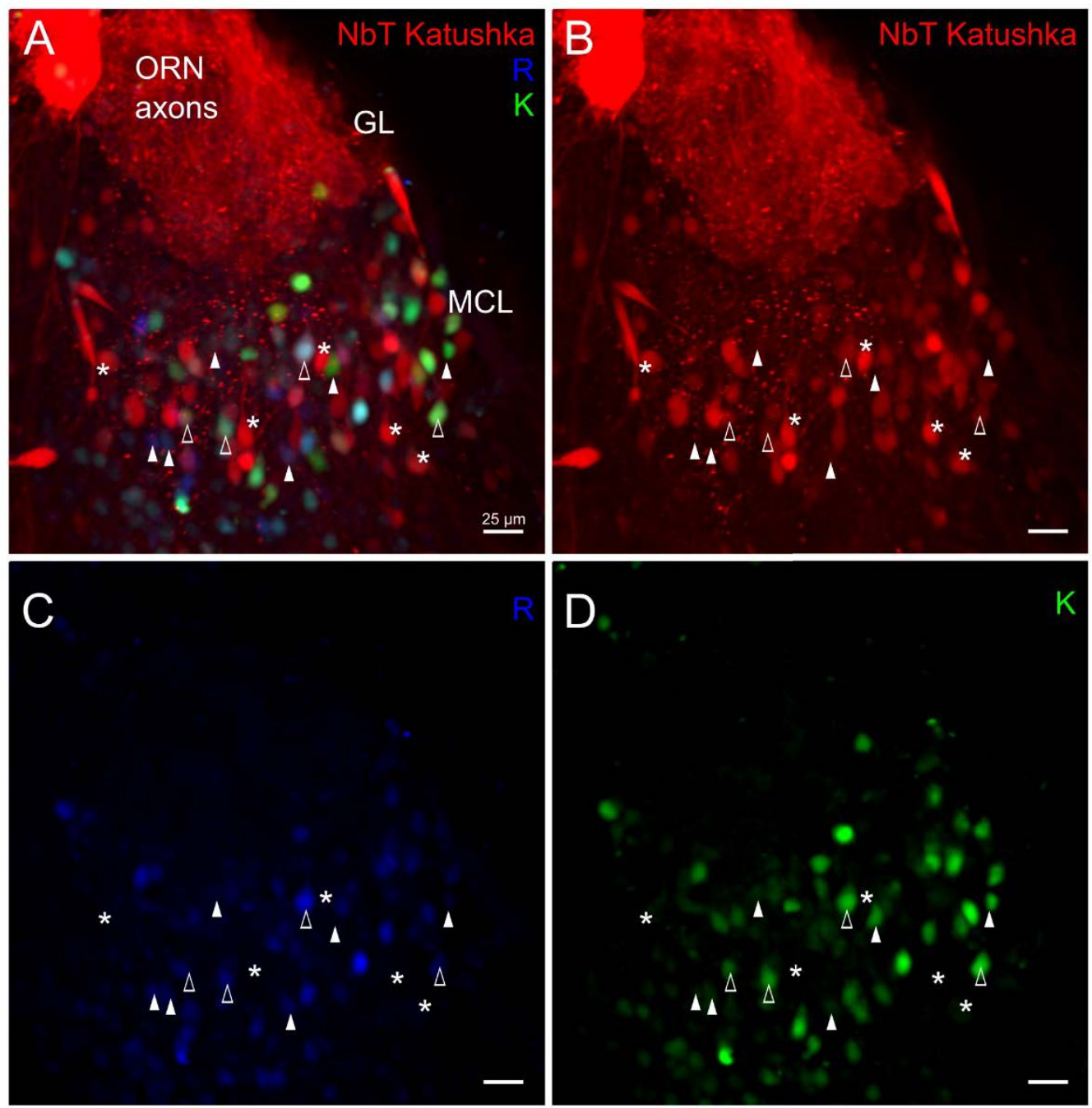

Figure $7 \mathrm{NbT}$ promotor activity and odor responses to amino acid stimuli in NbT-positive MTCs of the LC

A, B, C, and D) Fluorescence intensity difference map of odor responses in the OB neuronal network in NbTKatushka animals. Katushka fluorescence (red) is visible in ORN axons and a set of MTCs in the MCL. Odor responses to the amino acids, arginine ( $C$, blue) and lysine ( $D$, green) can be found in NbT-positive (white empty arrowheads) and NbT-negative (white arrowheads) neurons of the MCL. NbT-positive neurons not responding to amino acids (B, C, and D; white asterisks) were also present in all animals imaged.

\subsection{GLOMERULI, JGCS AND MTCS PREFERENTIALLY RESPOND TO DEFINED GROUPS OF AMINO ACIDS AND SHOW DIFFERENTIAL TUNING TO AMINO ACIDS' INDIVIDUAL MOLECULAR FEATURES}

Loading of MTCs with Fluo-4 AM calcium indicator could be used to image the postsynaptic glomerular responses of MTC dendritic tufts. JGCs and other neurons of the MCL were also labeled by the MCBL method. To get insights into the organization of the glomerular odor map, I measured calcium responses of the postsynaptic glomerular network in the GL of larval Xenopus. To better visualize glomerular territories, the GL was beforehand labeled with WGA-Alexa Fluor 594 as described in the methods. The dataset was pooled from 10 animals' right ventral $\mathrm{OB}$ (ventromedial lobe of the LC). After thresholding and quality control of the spatial and temporal components (as described in the methods and the code) reactive ROIs (1256, 270 amino acid-responsive, glomeruli and 140 JGCs) were analyzed in total from 10 
animals. Relevant features analyzed were their cross-sectional area in $\mu \mathrm{m}^{2}$, positional information in the 3D image stack, and their deconvolved or raw fluorescence intensity time traces. Amino acid-responsive ROIs detected by the CalmAn algorithm underwent several levels of quality control and were manually assigned to the categories: glomeruli, JGCs or MTCs based on the criteria described in the methods section.

I performed a pair-wise correlation analysis between the pooled maximum response amplitudes of JGCs or glomeruli to individual amino acids (Figure 8, A). Response peak amplitudes to structurally related amino acids were weakly correlated ( $p: 0,2-0,6)$ or uncorrelated (Figure 8, C and D). Positive correlation coefficient values occurred between amino acids of molecularly related sidechains, like lysine and arginine (Figure 8, B). Between structurally different amino acids, for example arginine and tryptophan, the response amplitudes were either uncorrelated or negatively correlated (Figure 8,C and D). In general, the correlation matrices of the postsynaptic glomeruli and JGC somata were very similar. Correlation coefficient values (Pearson) between amino acid pairings of the different subgroups were either negative (Figure 8, C; JGCs) or values around zero (Figure 8, D; glomeruli). A clear preferential tuning of both glomeruli and JGCs to one of the amino acid mixes I used was apparent. Glomeruli and JGCs were either more selectively tuned to the mix of basic/aromatic amino acids, or the mix of amino acids with long, neutral sidechains (Figure 8, C and D).

I performed (correlation-based) hierarchical cluster analysis of the maximum response amplitudes of amino acid-responsive JGCs or glomeruli. I could observe several recurrent response profiles among glomeruli and JGCs. These clustered profiles could be grouped by dominant response amplitudes to either one, two, or three individual amino acids (Figure 8, C and D).

Analyzing the somatic responses of NbT-positive and NbT-negative neurons from the OBs of seven NbT-Katushka tadpoles, similar clustering patterns were observed using correlationbased hierarchical cluster analysis. NbT-positive and NbT-negative neurons could be coarsely grouped by dominant responses to one, up to three amino acids (Figure 9, C and D). However, the strict selectivity between amino acids subgroups (basic/aromatic vs. longchain-neutral mixtures), as observed on the glomerular level, was not as pronounced on the $\mathrm{MCL}$ level (Figure 9, A and B). Correlation coefficients between maximum response amplitudes to single amino acids above 0,25 were sparse and only occurred between structurally related amino acids like arginine and lysine or tryptophan, histidine, and phenylalanine (Figure 9, A and B; see Figure 8, B). 


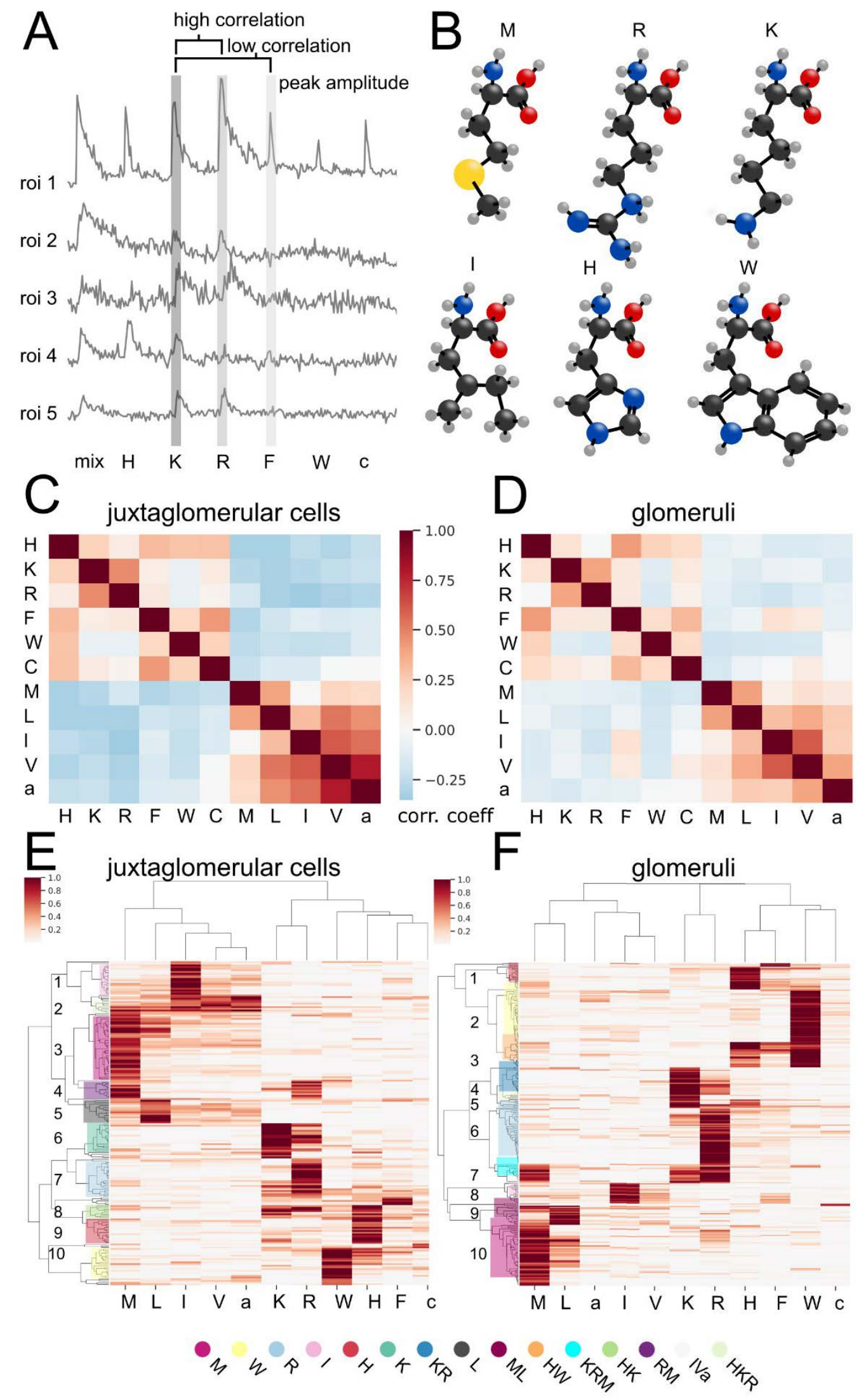

Figure 8 Selective tuning profiles to single amino acids' structural features in glomeruli and JGCs

A) Analysis of correlation between stimulus-induced response peak amplitudes. Maximum response amplitudes of all responsive ROls were pooled and used for calculation of Pearson's correlation coefficient (number of glomerular ROIs: 270; number of JGCs: 140; number of animals: 10) B) Molecular structures of a set of L-amino acids used (M, R, K, I, H and W; hydrogen (grey), carbon (black), nitrogen (blue), oxygen (red) and sulfur (yellow) atoms). C, D) Correlation-matrices (Pearson's correlation coefficient; see color scale) of JGCs' (C) or glomeruli's (D) maximum response amplitudes to single amino acid stimuli. E, F) Hierarchical cluster analysis (correlationbased) of pooled peak response amplitudes to single amino acid stimuli. Odorant response profiles of 
juxtaglomerular cells (E) and glomeruli (F) cluster into groups exhibiting prominent responses to single amino acids or few structurally related amino acids (single letter code combinations; different colors).

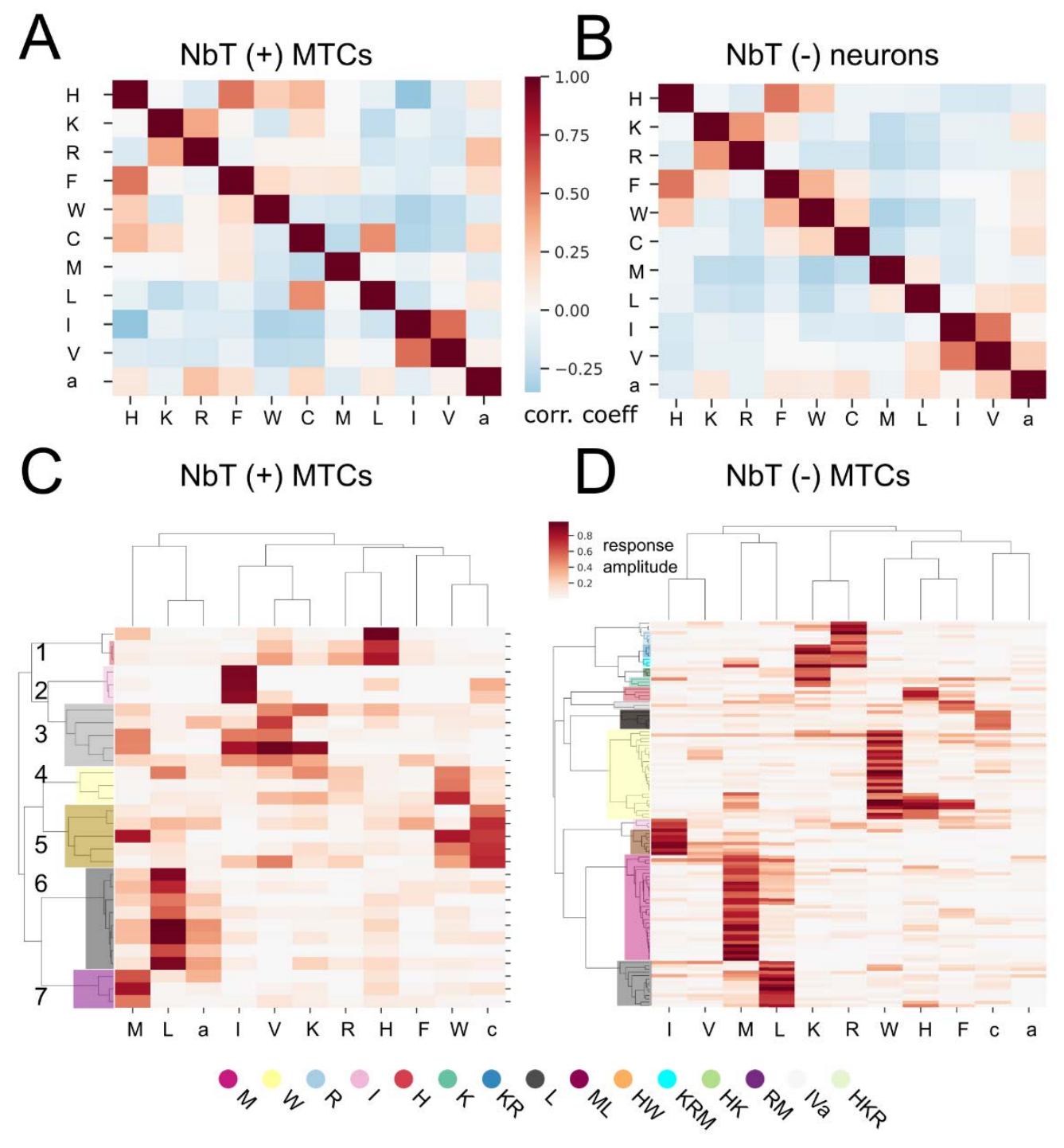

Figure 9 Odor response profiles to amino acid stimuli in NbT-positive and negative neurons

A, B) Analysis of correlation between stimulus-induced response peak amplitudes. Maximum response amplitudes of all responsive NbT-positive MTCs or NbT-negative neurons were pooled and used for calculation of Pearson's correlation coefficient (number NbT-positive MTCs: 30; NbT-negative neurons: 117; number of animals: 7). Correlation-matrices (Pearson's correlation coefficient; see color scale) of NbT-positive MTCs (A) or NbT-negative neurons (B) maximum response amplitudes to single amino acid stimuli. C, D) Hierarchical cluster analysis (correlation-based) of pooled maximum response amplitudes to single amino acid stimuli. Odorant response profiles of NbT-positive MTCs (C) and NbT-negative neurons (D) cluster into groups showing prominent responses to single or few structurally related amino acids (single letter code combinations; different colors).

Based on the observed clustering in hierarchical cluster analysis, I grouped odor response profiles according to their dominant response amplitudes to certain amino acids. Fluorescence intensity values of each responsive ROI were normalized to the maximum response amplitude (1.0). The (dominant) odor tuning of each responsive ROI will from now on be defined as the (group of) amino acid stimuli that trigger responses with an amplitude of more than $50 \%$ of the maximum response amplitude observed in that entire measurement (Figure 10; A). This (dominant) odor tuning is represented by a combination of the amino acid single letter code and a particular color throughout the thesis (Figure 10; A). 
A
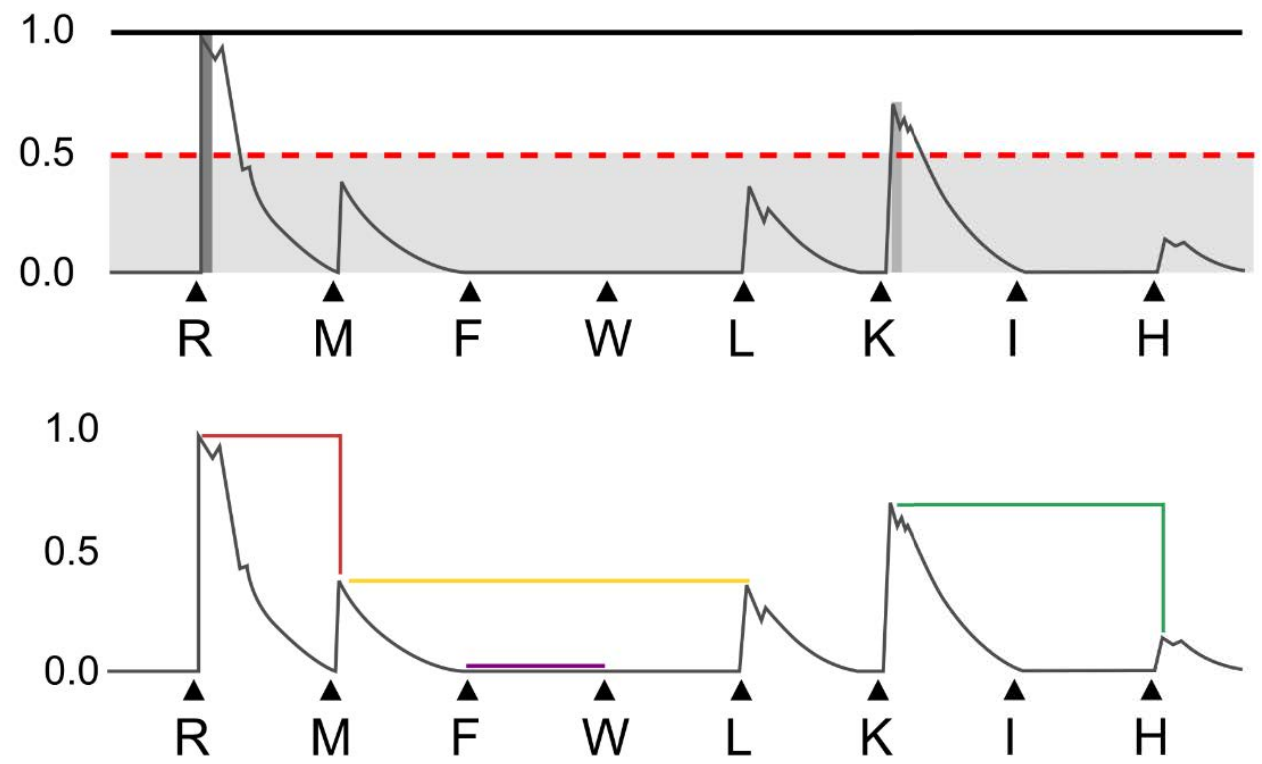

B

dominant odor tuning

( $50 \%$ of maximum peak amplitude)

KR

relative peak amplitude differences

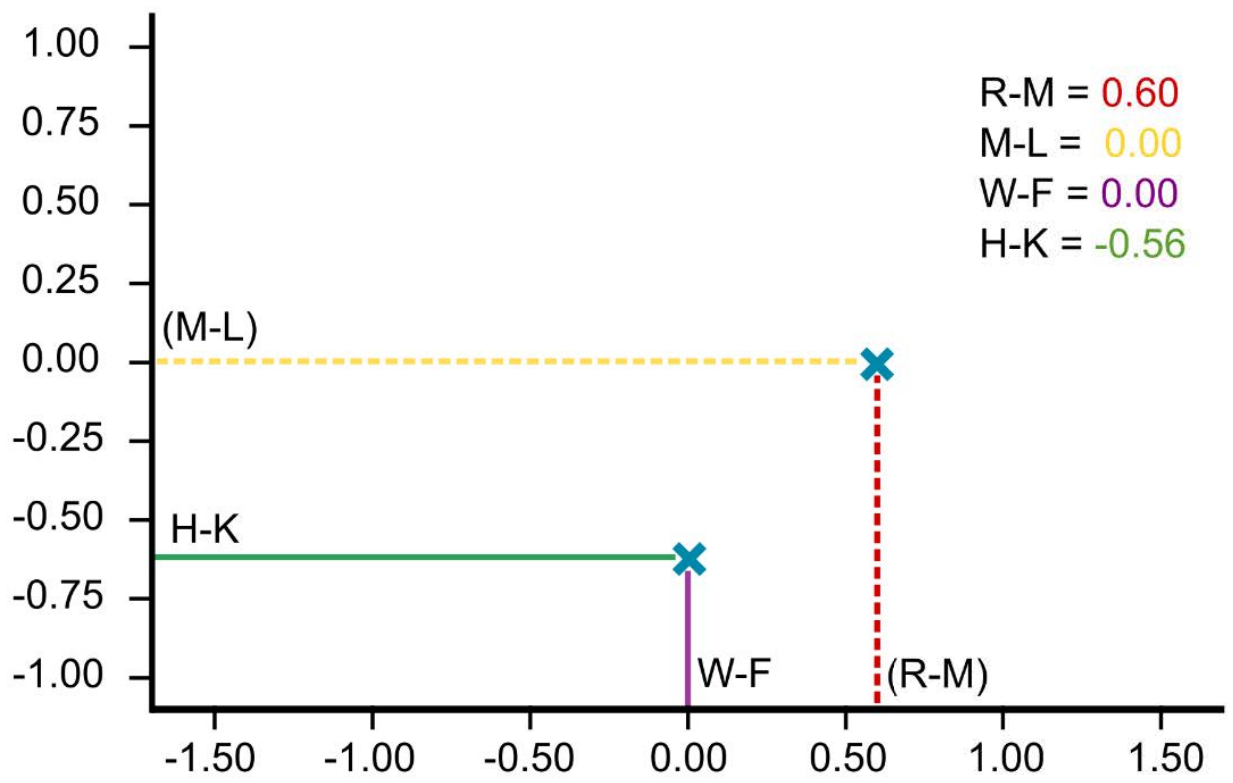

Figure 10 Categorization of odor response profiles by their tuning to dominant stimuli and relative response amplitude differences

A, B) Definition of (dominant) odor tuning (upper panel) and relative response amplitude differences of subthreshold stimulus responses (lower panel). A) Dominant odor tuning of ROls to single amino acid stimuli was defined as the (combination of) stimuli that exceeded $50 \%$ of the maximum odor response of the entire measurement (see upper example trace; maximum response of the measurement: arginine $(R)=1.0$; dominant odor tuning: KR since response peak to lysine (K) exceeds $50 \%$ of response peak amplitude to $\mathrm{R}$; red dashed line). Relative differences in response amplitudes were calculated by subtracting peak response amplitudes to individual amino acids from each other (A; lower panel). B) Relative differences in response amplitudes to pairings of amino acid stimuli (B, upper right panel) were used to map ROIs on a 2D cartesian coordinate system. Graphs like this one contain information of both (dominant) odor tuning (KR; individual color of datapoint) and relations of 
subthreshold responses to each other (position on coordinate system f. ex. $x=W-F$ and $y=H-K$ or $x=R-M$ and $y=M-L)$.

Responsive glomeruli, JGCs and MTCs could be grouped by this approach according to their odor selectivity. However, the individual response profiles were still variable when taking into account odor responses with amplitudes below $50 \%$ of the maximum response amplitude.

To visualize the (dominant) odor tuning and variability in response amplitudes to other stimuli, I plotted responsive ROIs (glomeruli, JGCs, MTCS) in a way, that the $x$ - and $y$-axis represented the difference in response peak amplitudes between two amino acid responses in the respective measurement (Figure 10; B). These relative differences in peak amplitude between two odor stimulus responses (Figure 10; A, lower panel; Figure 10; B) were used to assign the entity of responsive ROls to 2-dimensional cartesian coordinate systems. Their (dominant) odor tuning, type, and cross-sectional area are represented by individual colors, symbols and the symbols' sizes (Figure 11, Figure 12).

Glomeruli and JGCs of same (dominant) odor tuning were variable in relative response amplitude differences to other amino acid combinations (Figure 11, A and B). This range of variability became apparent through the extent of spatial clustering of ROIs with identical (dominant) odor tuning. The variability in the individual response profiles of glomeruli and JGCs was linked to their (dominant) odor tuning. For instance, glomeruli with (dominant) odor tuning to tryptophan were biased to exhibit higher response amplitudes to histidine than to lysine (Figure 11, A). Glomeruli with (dominant) odor tuning to lysine, on the other hand, were biased to exhibit higher response amplitudes to arginine than to tryptophan (Figure 11, A). This bias was correlated with the structural similarity of the individual amino acid residues. Relative response amplitude differences of zero indicated that some glomeruli or JGCs did not have any response to the two given amino acids at all, or the response amplitudes were identical (Figure 10; A, lower panel; Figure 11, A and B). Either way, this indicated an indifference in odor tuning to the selected amino acids' differences in molecular structure (Figure 10; A, lower panel, Figure 12). Overall, glomeruli, JGCs and MTCs were tuned selectively to individual amino acids or combinations of structurally similar amino acids (dominant odor tuning). Smaller response amplitudes, especially the relative amplitude differences between stimuli, varied but were linked to the structure of the dominant amino acid stimuli. 


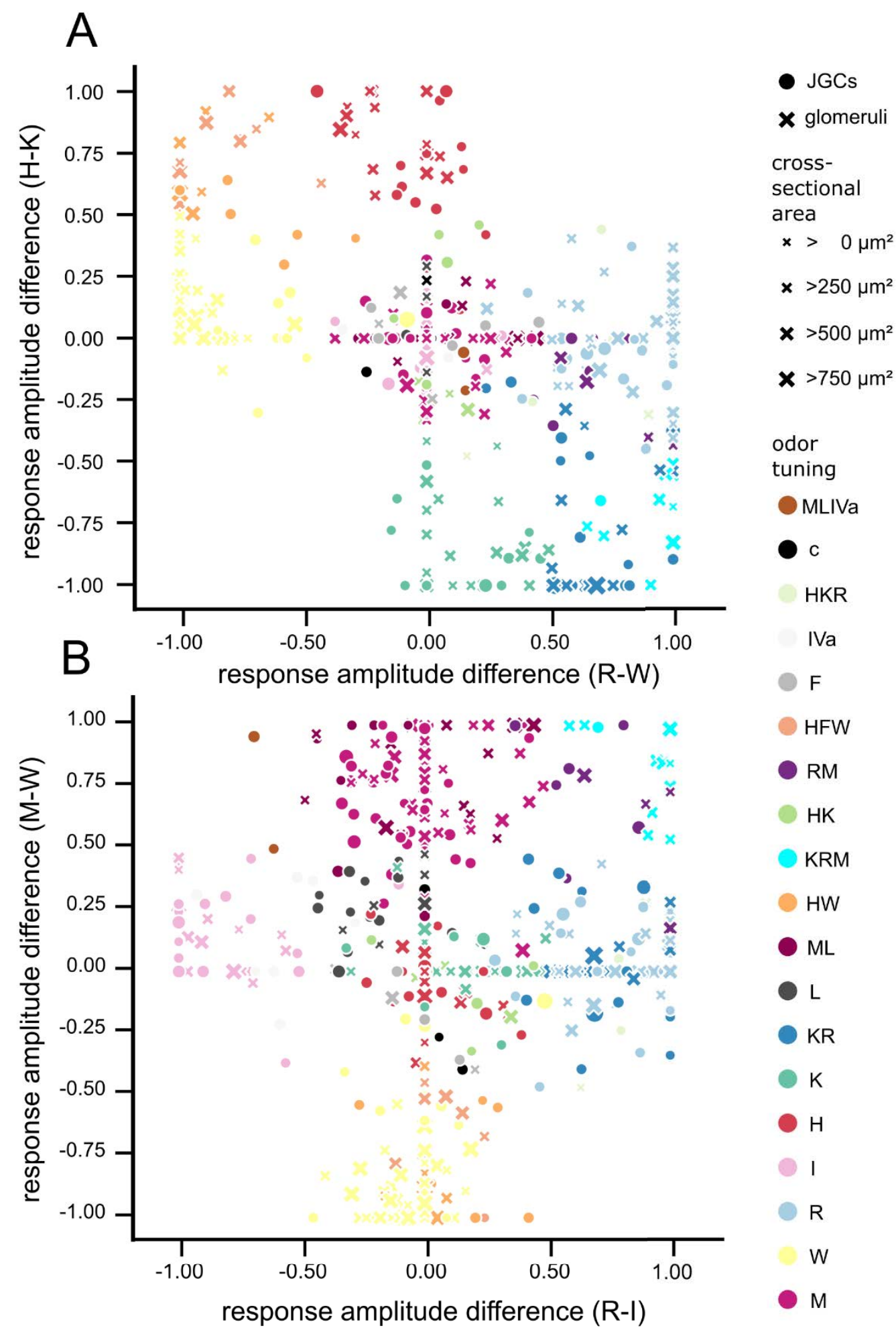

Figure 11 Odor tuning and relative differences in response amplitudes of glomeruli and JGCs to amino acids

A and B) JGCs (circles) and glomeruli (crosses) plotted according to their relative differences in response amplitudes to the amino acid pairings R-W and H-K; details see Figure 10). Glomerular cross-sectional areas are indicated by the crosses' relative sizes. Colors and single-letter codes represent the (dominant) odor tunings. Glomeruli and JGCs of identical (dominant) odor tunings spatially cluster. Variance in response amplitude differences were apparent among glomeruli and JGCs of identical odor tuning. For several clustered groups with identical odor tuning, uni-lateral distribution to structurally similar amino acids were observed. (number of glomeruli: 270; number of JGCs: 140; 10 animals) 

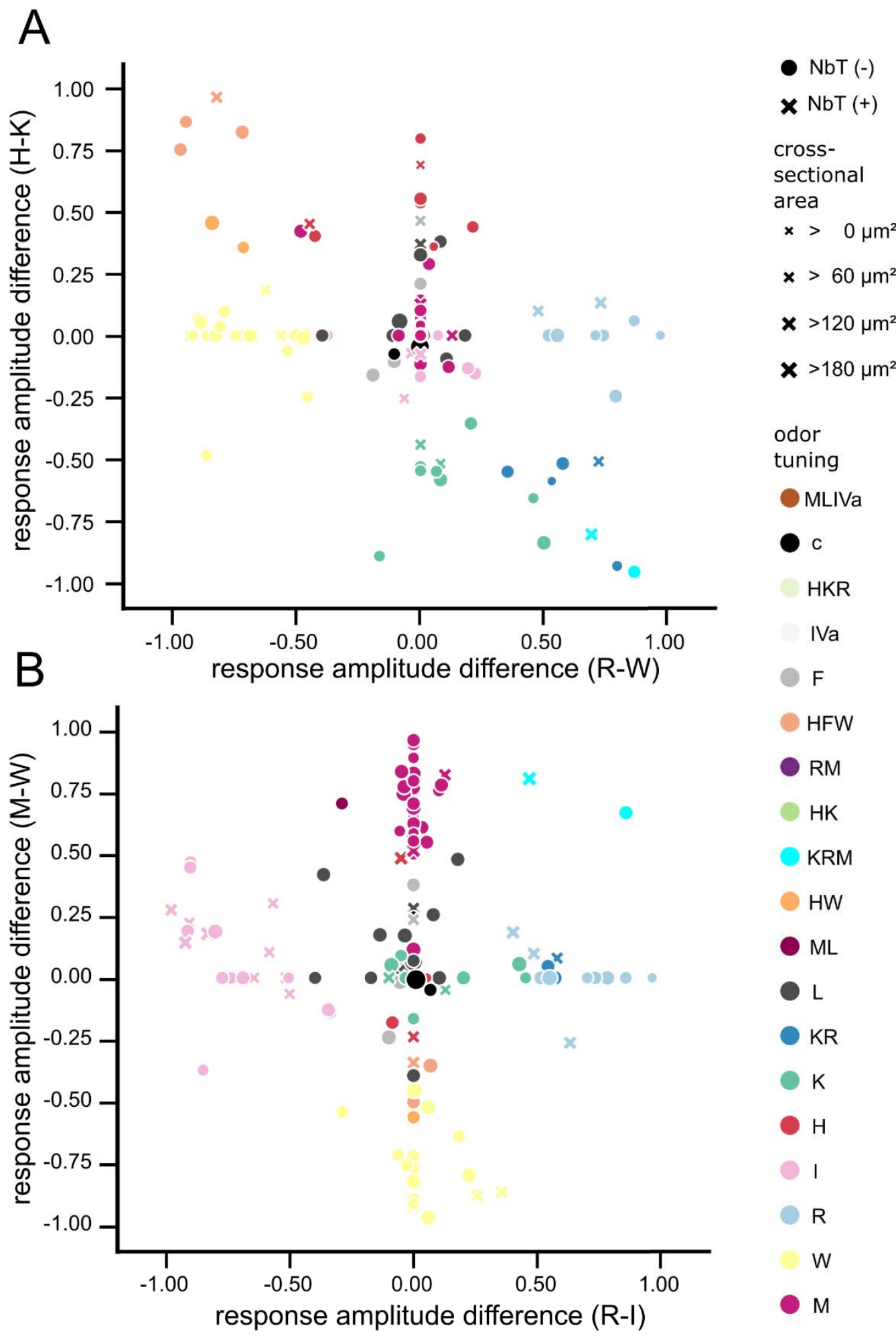

Figure 12 Odor tuning and relative response amplitude differences in NbT-positive MTCs and NbT-negative neurons of the $M C L$

A and B) NbT-negative neurons (circles) and NbT-positive MTCs (crosses) plotted according to their relative differences in response amplitudes to the amino acid pairings $\mathrm{R}-\mathrm{W}$ and $\mathrm{H}-\mathrm{K}$; details see Figure 10). NbT-positive MTCs' cross-sectional areas are indicated by the crosses' relative sizes. Colors and single-letter codes represent the (dominant) odor tunings. Neurons of identical (dominant) odor tunings spatially cluster. Variance in response amplitude differences were apparent among neurons of identical odor tuning. For several clustered groups with identical odor tuning. (number NbT-positive MTCs: 30; NbT-negative neurons: 117; number of animals: 7) unilateral distribution to structurally similar amino acids were observed. 
The numbers of different odor tunings present in glomeruli, JGCs, and MTCs, were highly dependent on the response amplitude threshold used. To validate the suitability of the $50 \%$ response threshold I used, I compared the classification by odor tuning to dominant stimuli with the fluorescence intensity difference map created from CalmAn movies (See methods section). The glomerular activity map created from positional information of the individual PGCs or glomeruli and their (dominant) odor tuning was very comparable to the one based on fluorescence intensity difference images (Figure 13). Some glomeruli that were visible in the intensity difference map were not present in the odor map created with CalmAn and the dominant odor tuning thresholding. This was often due to the strict criteria I defined for the selection process of responsive ROIs and their odorant response profiles.
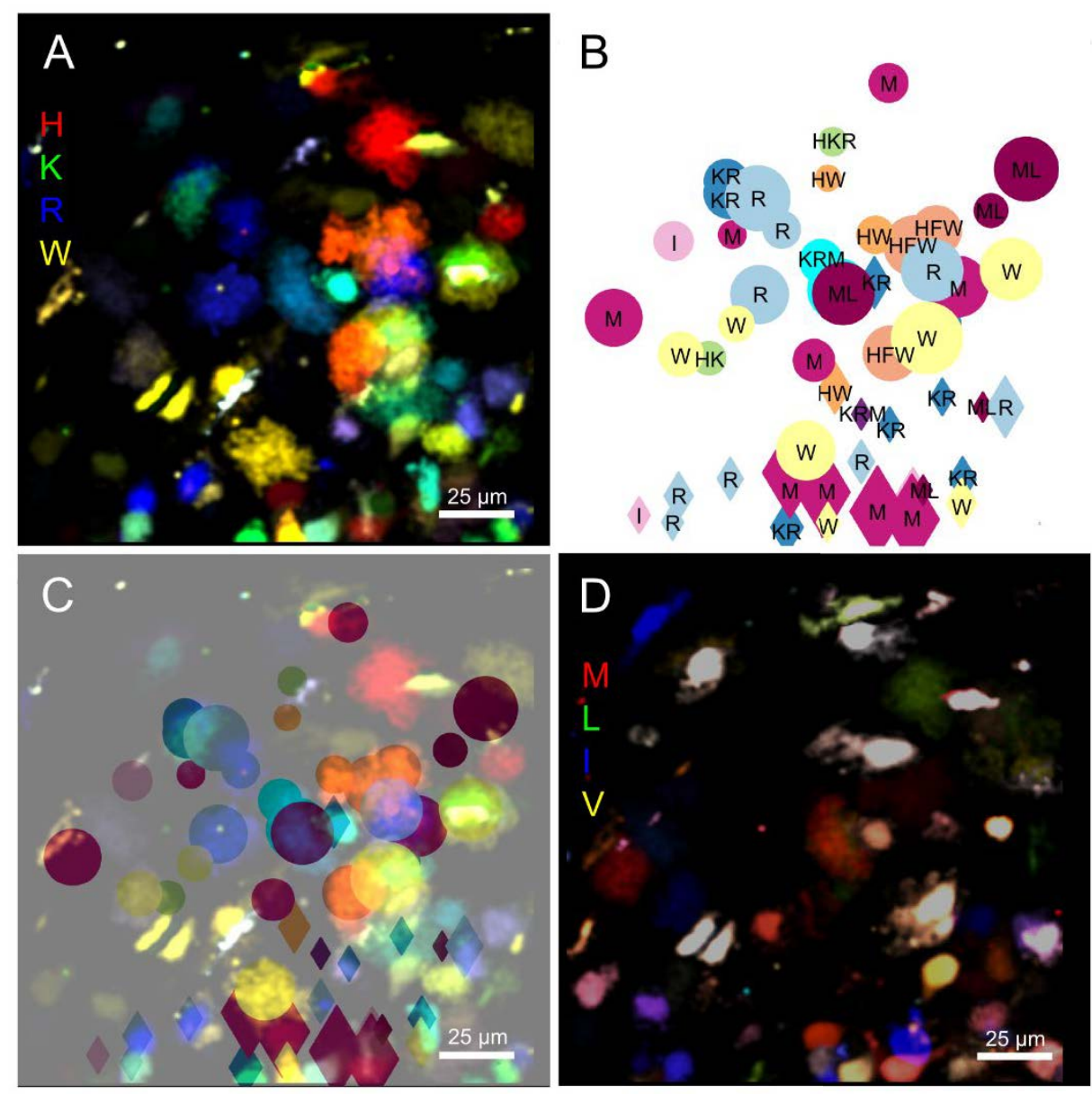

Figure 13 Comparison of threshold-based odor tuning with fluorescence intensity difference maps of glomerular activity

A) Fluorescence intensity difference map of the medioventral LC to a set of single amino acids (for detailed description of difference map generation see methods section). Glomerular responses to amino acids are depicted as individual colors $(\mathrm{H}$ : red, $\mathrm{K}$ : green, R: blue and W: yellow). Mixed colors from those primaries (f.ex. orange and cyan glomeruli) represent glomeruli responsive to several amino acids. B) Glomerular odor map of the same animal as in (A). Responsive ROIs and their coordinates were detected by the CalmAn algorithm. Dominant odor tuning (colors and single-letter code) of glomeruli (circles) and JGCs (diamonds) using the 50\% threshold (Figure 10, A) matched the intensity-based odor maps. C) Overlay of both odor map methods shows high congruence of glomerular position, size and tuning. D) Fluorescence intensity-based odor map of the same animals medioventral LC to four different amino acids (M: red, L: green, I: blue, V: yellow). 
From the 1223 amino acid-responsive ROIs from all the postsynaptic glomerular calcium imaging experiments, I selected only ROIs of 20 different odor tunings for further analysis $(n=831)$. The chosen odor tunings included the 18 most frequent odor tunings in the dataset (tunings with positive responses to the alcohol mix excluded). Additionally, ROls with tuning profiles selective to all amino acids of either the basic/aromatic or long-chain-neutral amino acid mix were analyzed (Figure 14; C). The majority of glomeruli and JGCs were tuned to single amino acid stimuli. In both glomeruli and JGCs, odor tunings to methionine, tryptophan, arginine, isoleucine, lysine and histidine were most common (Figure 14; A, E and F). While for some odor tunings, the numbers of glomeruli and JGCs were similar, especially the arginine and tryptophan reactive glomeruli were more frequent in number than JGCs of the same tuning (Figure 14; B, D).

The amino acid-responsive ROls from the calcium imaging experiments in the MCL of NbTKatushka animals were subdivided into the 20 odor tuning profiles as described before $(n=$ 540). A significant difference between the two datasets was that many neurons of the MCL 'failed' the strict selection criteria for ROIs that were necessary for further analysis (Figure 15, C). The disqualification of ROIs for analysis was mainly due to high levels of noise or superimposed spontaneous activity in addition to the odor induced calcium transients. In these somatic measurements, the vast majority of NbT-positive MTCs and other NbTnegative neurons of the MCL were also tuned to individual amino acids (Figure 15, A, D, E and F). While NbT-positive MTCs represented only a fraction of the measured neurons (Figure 15, B), the most frequent odor tuning profiles to amino acids were present in this population (Figure 15, D, E).

Interestingly more NbT-positive MTCs were responsive to long-chain-nuclear amino acids (isoleucine, leucine, methionine) than to basic-aromatic amino acids (Figure 15, E). On the glomerular level, tryptophan and arginine responsive glomeruli were very frequent (Figure 14; E). However, it needs to be taken into account that the number of amino acid-responsive NbT-positive MTCs analyzed was lower compared to the number of glomeruli. 

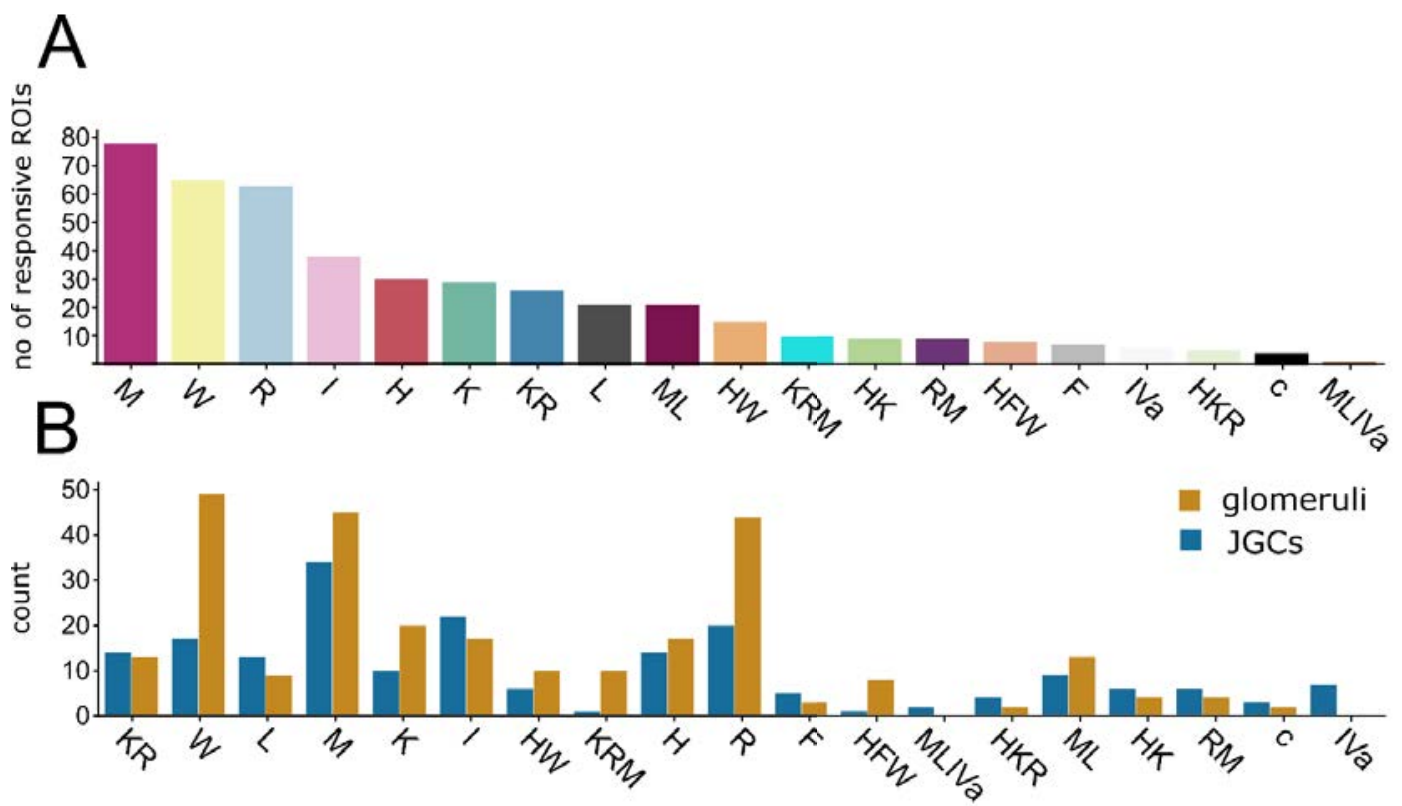

C Odor tuning of responsive ROIs

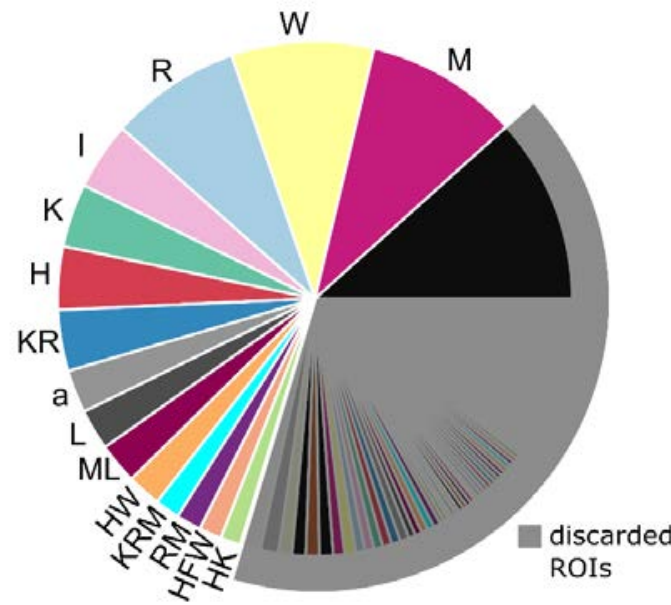

E Glomerular population odor tuning

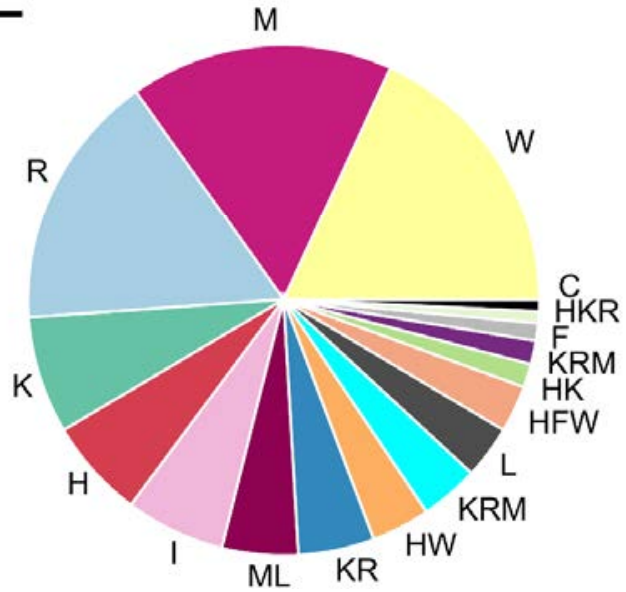

D Odor tuning glomeruli vs. JGCs

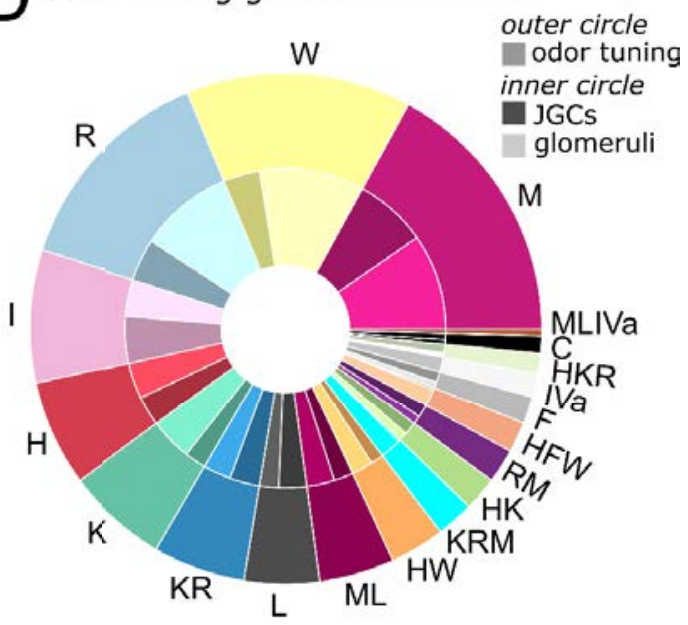

F JGC population odor tuning

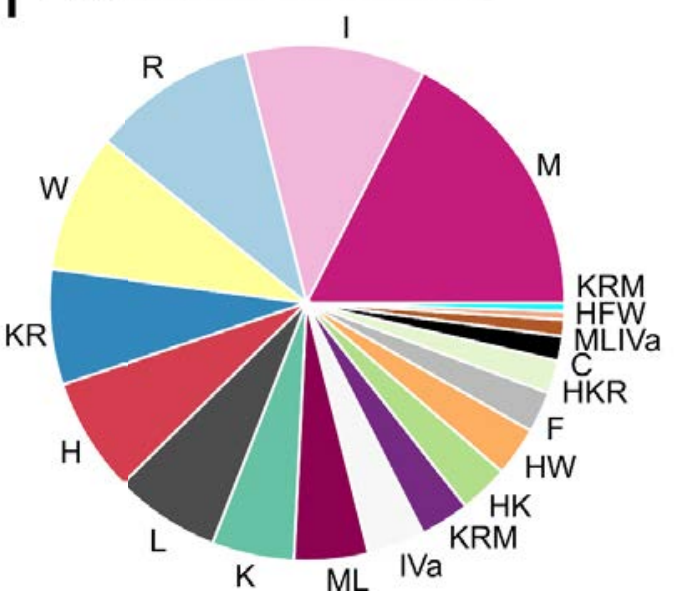

Figure 14 Frequency of the most common odor tunings of glomeruli and JGCs

A) Most common (dominant) odor tunings to amino acids (of both glomeruli and JGCs) sorted by frequency. B) Numbers of glomeruli (orange) vs. number of JGCs (blue) displaying individual odor tunings (number of glomeruli: 270; number of JGCs: 140; 10 animals). C) Frequency of odor tunings in all responsive ROIs. ROls discarded due to quality control (black) and sub-selection of most frequent profiles are shaded grey. C) Nested pie-chart of odor response profile distribution in general (outer circle) and the ratio of the respective tunings (inner circle) between glomeruli (lighter color variant) and JGCs (darker color variant). E, F) Individual frequencies of odor tunings in glomeruli (E) and JGCs (F) 


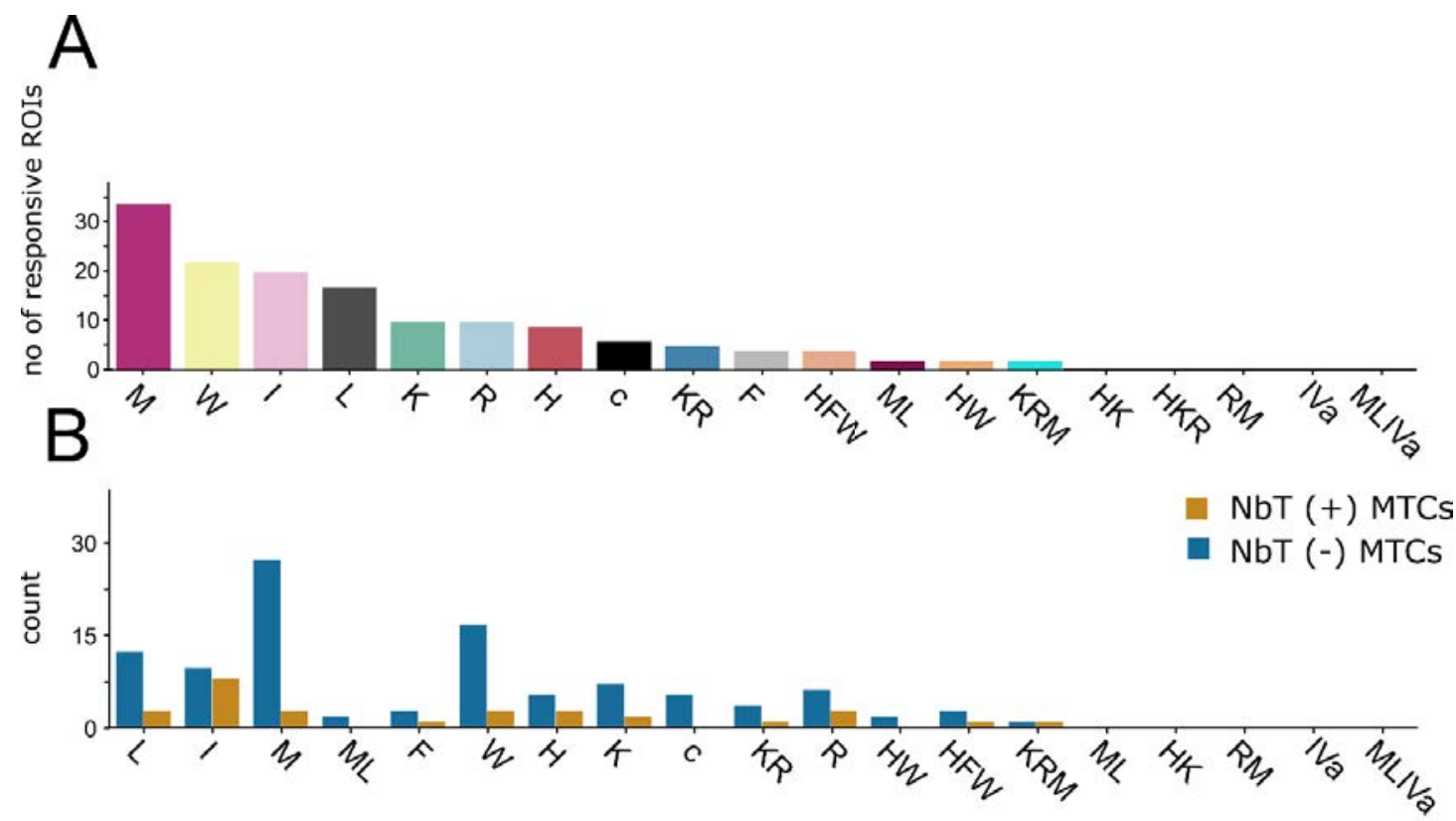

C Odor tuning of responsive ROIs

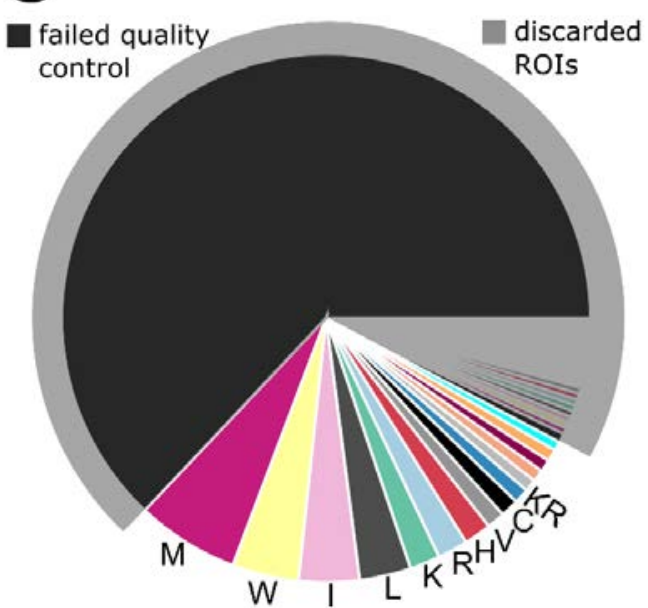

E NbT (+) MTC population odor tuning

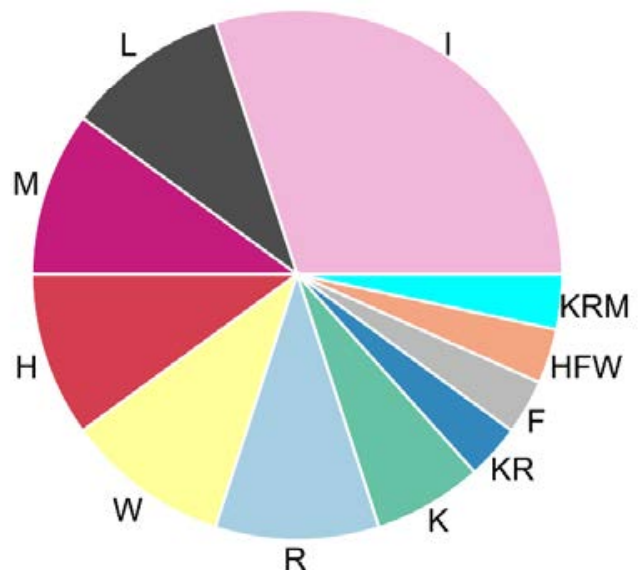

D Odor tuning $\mathrm{Nbt}(+)$ vs (-) MTCs

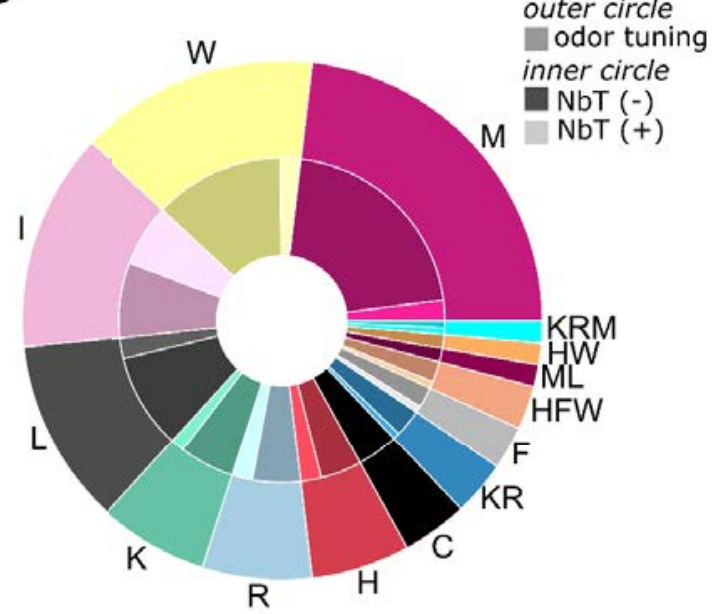

NbT (-) MTC population odor tuning

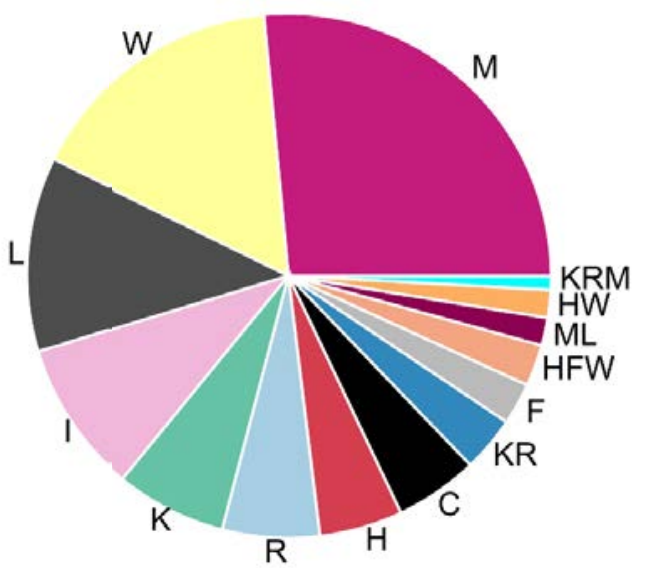

Figure 15 Frequency of the most common odor tunings in NbT-positive MTCs and NbT-negative neurons

A) Most common (dominant) odor tunings to amino acids (of NbT-positive MTCs and NbT-negative neurons) sorted by frequency. B) Numbers of NbT-positive MTCs (orange) vs. NbT-negative neurons (blue) displaying individual odor tunings (number of glomeruli: 270; number of JGCs: 140; 10 animals). C) Frequency of odor tunings in all responsive ROIs. ROIs discarded due to quality control (black) and sub-selection of most frequent profiles are shaded grey. C) Nested pie-chart of odor response profile distribution in general (outer circle) and the ratio of the respective tunings (inner circle) between NbT-positive (lighter color variant) and NbT-negative neurons (darker color variant). E, F) Individual frequencies of odor tunings in NbT-positive MTCs (E) and NbT-negative neurons $(F)$ 


\subsection{HETEROGENEITY IN SIZE OF AMINO ACID RESPONSIVE GLOMERULI}

After I characterized the odor tuning of glomeruli in the LC, I used the sizes of the spatial components detected by the CalmAn algorithm to draw conclusions about sizes of functional glomeruli in larval Xenopus. The cross-sectional areas of amino acid-responsive ROls were used as a measure of glomerular and JGC somatic sizes. Most JGC somata were in the range of 50-200 $\mu \mathrm{m}^{2}$ (assuming a circular shape: diameters $8 \sim 16 \mu \mathrm{m}$ ). JGC sizes were similar between ventral and dorsal sections of the bulb (Figure 16; A and C; mean cross-sectional area of all JGCs $120.5 \mu \mathrm{m}^{2}$ ). Glomeruli of the larval OB were very variable in size. Some glomeruli were in the range of JGC somata, whereas other glomeruli had cross-sectional areas around $600 \mu \mathrm{m}^{2}$ (assuming a perfect circle: diameter 8 26 $\mu \mathrm{m}$; mean cross-sectional area: $246.1 \mu \mathrm{m}$ ). These sizes fit well with the observed glomerular morphologies in the Calcein tracings (Figure 6). The average cross-sectional area of glomeruli was $200 \mu^{2}$ or higher independent on their position along the dorsoventral axis (Figure 16; A). I could observe variability in glomerular size depending on the individual glomerular odor tuning (Figure 16; C). Especially glomeruli responding to combinations of histidine and lysine were smaller than tryptophan or arginine-responsive glomeruli on average (Figure 16; C). Glomeruli that responded to tryptophan or combinations, including tryptophan, were particularly variable in glomerular size (Figure 16; C). However, high variability in glomerular size was observable in most glomeruli.

\subsection{LACK OF STEREOTYPY IN THE GLOMERULAR ODOR MAPS BETWEEN ANIMALS}

A major feature that can give insight into olfactory system wiring logics is the glomerular odor map, i.e. the relative positioning of glomeruli to each other depending on certain molecular features. I used fluorescence intensity difference images to visualize responsive glomeruli to particular amino acid stimuli in multiple colors, as described in the methods section. While certain glomerular response profiles (colors) were present in the odor maps of most measured animals $(n=10)$, there was no apparent stereotypic distribution of glomeruli along the mediolateral or rostro-caudal axis (Figure 17; Figure 18, B, C, D and E). Glomeruli of individual odor tunings were variable in number between animals (Figure 17; A). I pooled the glomeruli of all animals and analyzed their distribution along the mediolateral (Figure 18, D), rostro-caudal (Figure 18, C) and dorsoventral axis (Figure 18, E). The distribution of individually tuned glomeruli was broad along all axes with no clear positional bias. The majority of glomeruli were scattered across the entire GL independent of their odor tuning (Figure 18, B). 
A

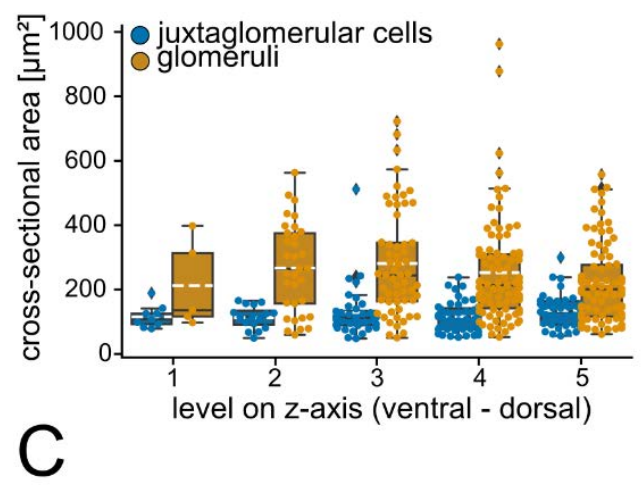

B

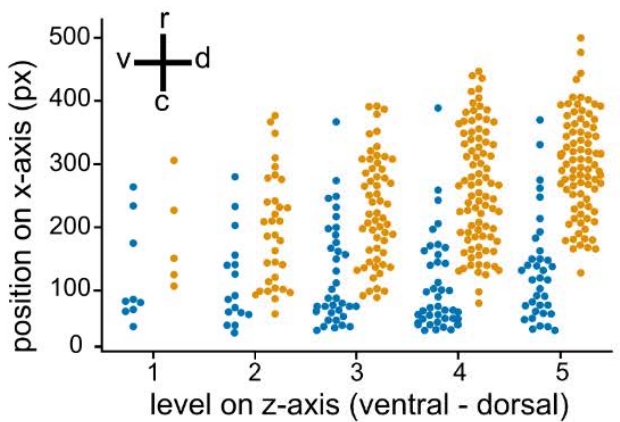

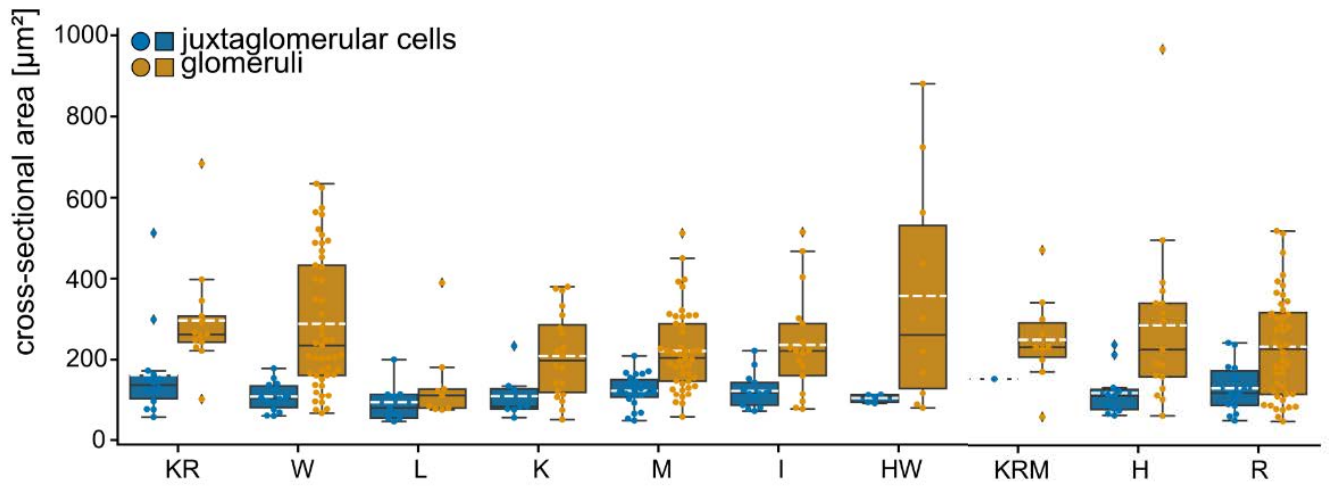

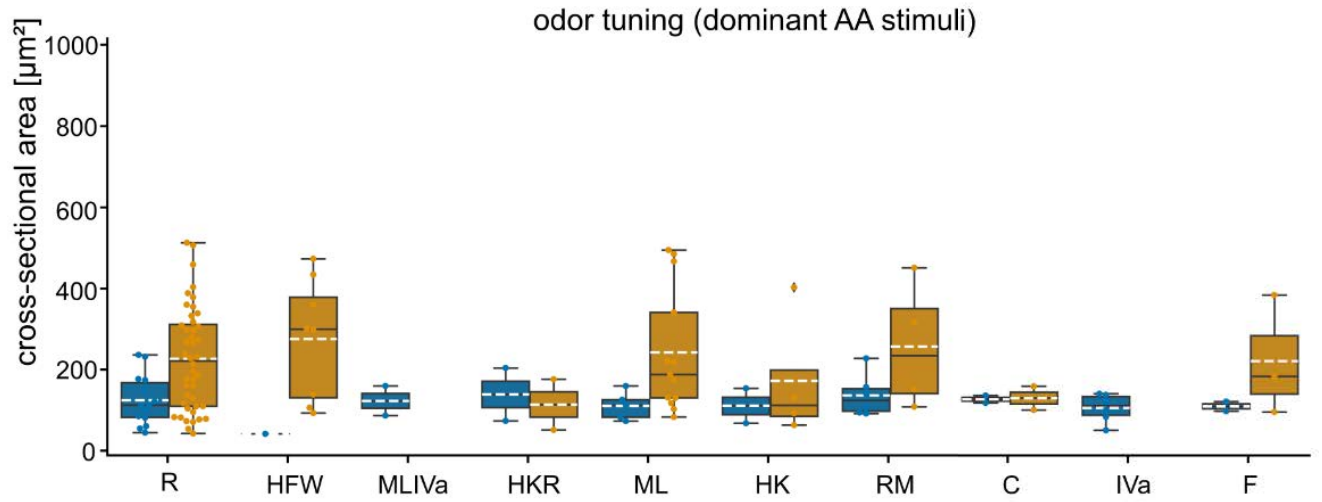

Figure 16 Sizes and spatial distribution of glomeruli and JGCs in the lateral larval OB

A) Distribution of glomerular (orange) and JGC (blue) sizes (cross-sectional areas in $\mu^{2}$ ) on 5 different levels along the dorsoventral axis in the larval OB. B) Spatial distribution of glomeruli and JGCs along the rostro-caudal and dorsoventral axes. C) Cross-sectional areas (sizes) of glomeruli and JGCs of different (dominant) odor tunings. Boxplot outlines represent first and third quartile of distribution. Black horizontal lines illustrate the median, white dashed-lines the mean value of the cross-sectional area distributions. 

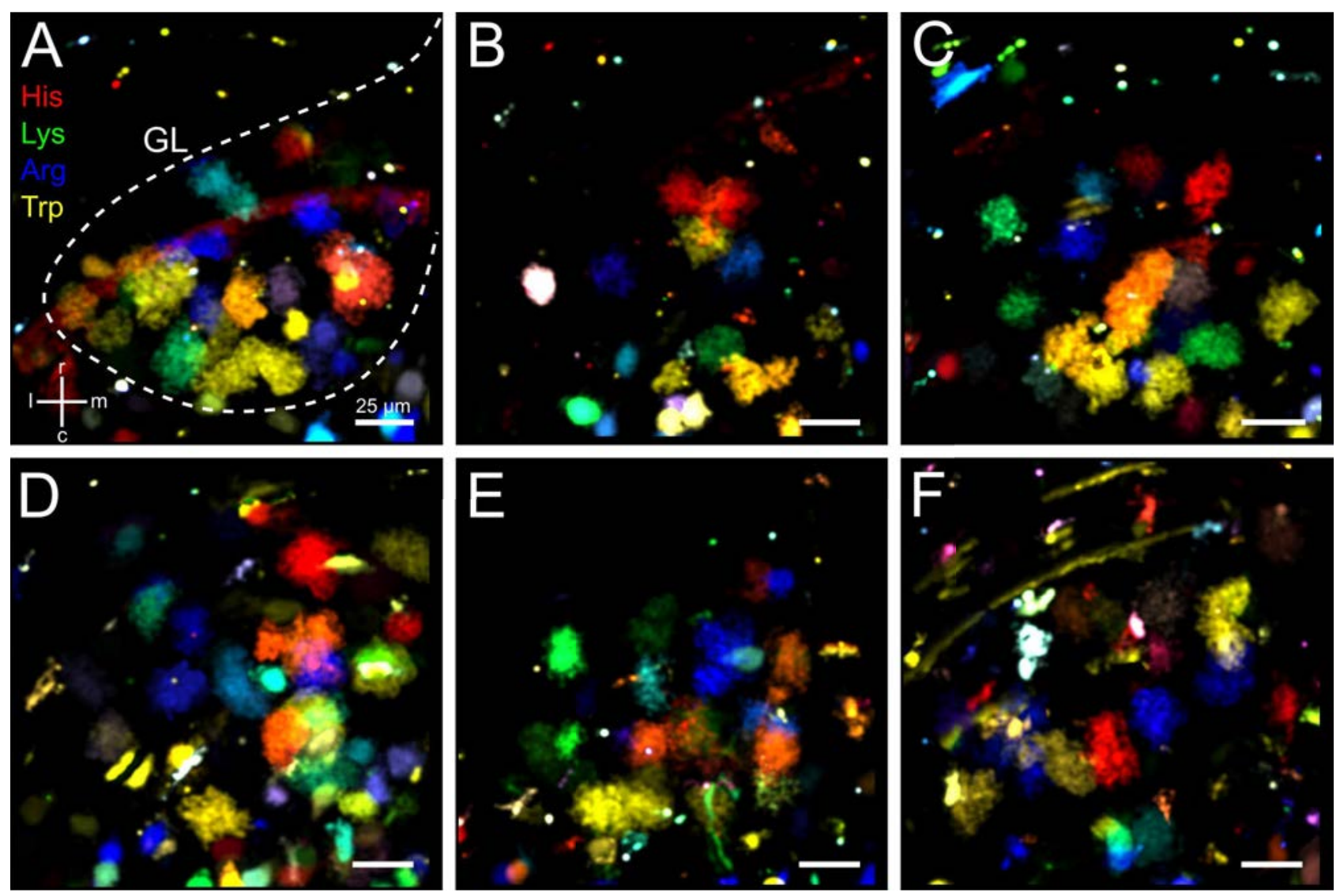

Figure 17 Non-stereotypical glomerular odor representation between animals in the LC

A - F) Glomerular odor maps (maximum projections of the measured medioventral LC) to amino acid stimuli $(\mathrm{H}$ : red, $\mathrm{K}$ : green, R: blue, W: yellow) in six representative animals (A-F). Variability in glomerular distribution and number of individually tuned glomeruli is visible by their activity (different colors). Scale bars: $25 \mu \mathrm{m}$ 

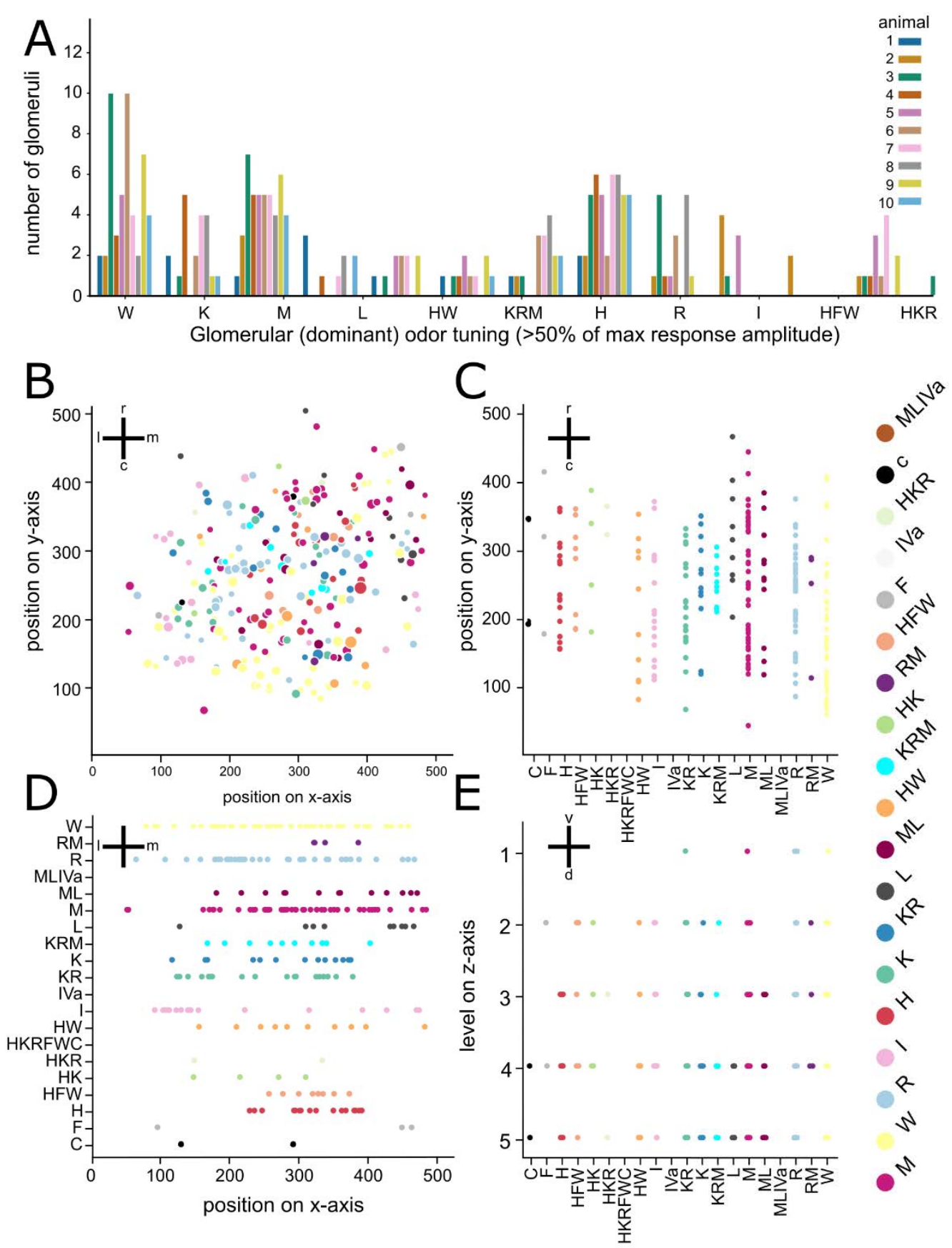

$\sqrt{2}$
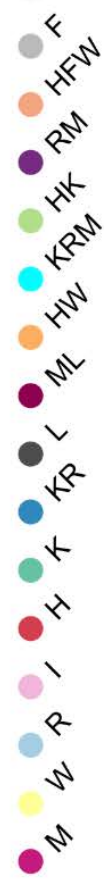

Figure 18 Variable number of glomeruli between animals and dispersed distribution of similary tuned glomeruli in the $L C$

A) Variability in number of glomeruli of individual odor tunings between animals (different colors; $n=10$ ). B, C, and D) Distribution of glomeruli (270 glomeruli pooled from 10 animals) along the rostro-caudal (B and C), mediolateral ( $B$ and $D$ ) and dorsoventral (E) dimension. Individual (dominant) odor tunings are indicated by colors and lettercodes.

\subsection{JUXTAPOSITION OF GLOMERULI IS NOT CORRELATED TO SIMILARITY IN ODOR TUNING}

To quantify further, whether chemotopy was indeed not present in the glomerular odor map of the LC, I analyzed the Euclidean distances between glomeruli in each individual animal. Moreover, the similarity in odor tuning was calculated between all pairings of glomeruli per animal (Figure 19; B, C). I used the Pearson correlation coefficient between the fluorescence 
intensity time traces as a measure of similarity in odor tuning. For every glomerular pairing, the Euclidean distance and correlation coefficient were calculated (Figure 19; B, C). The resulting data was pooled for general analysis. There was no correlation between the Euclidean distance of glomeruli to each other and their odor tuning (Pearson correlation coefficient between time traces; Figure $20, A$ ). In fact, glomeruli that were tuned to structurally different amino acids (for example, tryptophan and arginine) were often found in close proximity (juxtaposition) to each other (Figure 19, A). As an additional way to analyze the relation between odor tuning similarity and the inter-glomerular distance, I grouped the glomeruli into three categories based on their (dominant) odor tuning: Glomeruli with identical odor tuning, glomeruli that had at least one stimulus in common, and glomeruli that had no stimulus in common (Figure 20, C). There were no significant differences in distributions and mean Euclidean distance values between the three groups (Figure 20, C). Overall, the juxtaposition of glomeruli did not correlate with similarity in odor tuning. I also observed the independence of odor tuning and distance between individual JGCs (Figure 20, B)
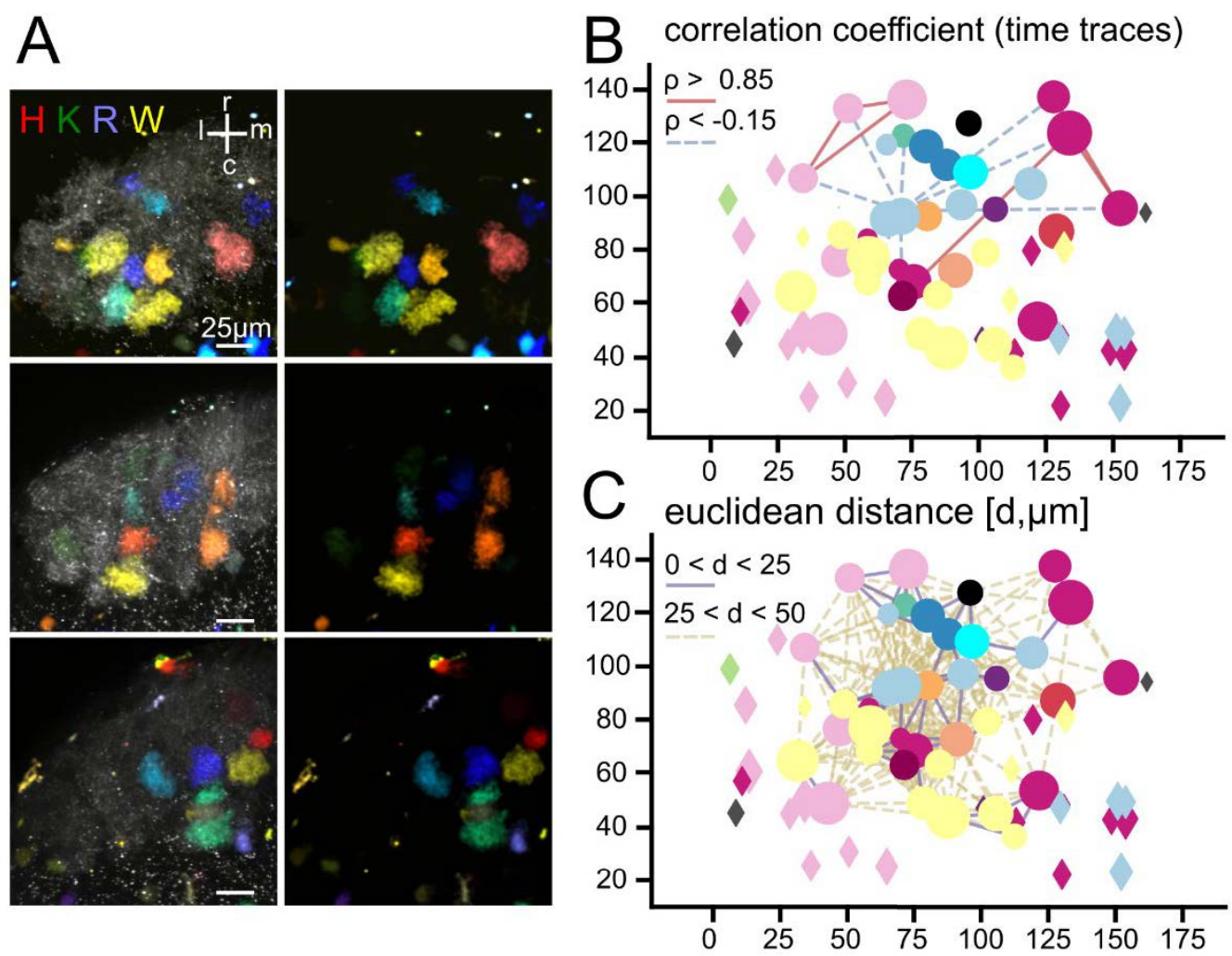

Figure 19 Quantification of glomerular juxtaposition and similarity in odor tuning

A) Glomerular odor maps of the medioventral larval LC. Glomeruli responsive to individual amino acids (H: red, $\mathrm{K}$ : green, R: blue, and W: yellow) in three different animals (three different rows). In the left column WGA-Alexa Fluor 594 labeling is included to visualize the glomerular array (grey punctuate staining). Glomeruli of different odor tunings (for example arginine (blue) and tryptophan-reactive glomeruli (yellow) appeared in close juxtaposition. B, C) Pairwise analysis of (B) glomerular (circles) odor response profile similarity (Pearson's correlation coefficient between fluorescence intensity time traces) and (C) inter-glomerular Euclidean distances. High odor tuning similarity (Pearson's correlation coefficient $p>0.85$ ) is indicated as red lines between glomeruli, uncorrelated activity $(\mathrm{p}<-0.15)$ as blue dashed lines. C) Euclidean distances between 0 and $25 \mu \mathrm{m}$ are indicated as magenta lines, distances between 25 and $50 \mu \mathrm{m}$ as yellow lines. JGCs: differently colored diamonds. 

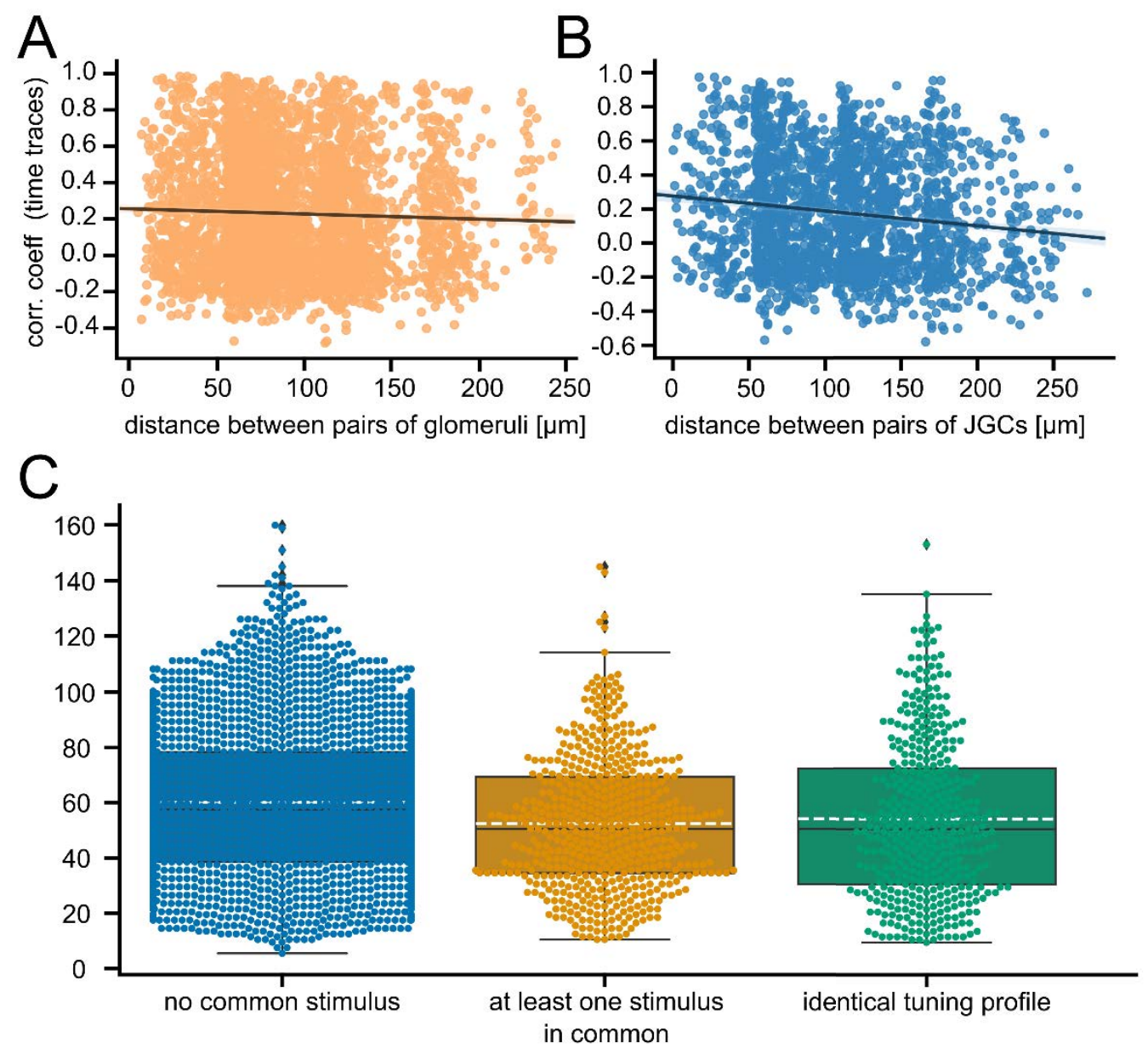

Figure 20 Lack of correlation between distance and similarity in odor tuning among glomeruli or JGCs

A) Pooled data of glomerular pairings (from 10 animals) with each dot (orange) representing a glomerular pair's inter-glomerular Euclidean distance and similarity in odor tuning (Person's correlation coefficient between fluorescence intensity time traces). No correlation between interglomerular distance and odor tuning similarity was apparent (fitted line). B) No correlation between JGCs' distances to each other and their odor tuning was apparent either. C) Glomerular pairings sorted by their similarity in (dominant) odor tuning and inter-glomerular distances. Three categories of similarity in (dominant) odor tuning were defined: no common amino acid stimulus in common, at least one stimulus in common or identical (dominant) odor tuning.

\subsection{JuXtaposition OF NBT-POSITIVE MTCS IS NOT CORRELATED to SIMILARITY IN ODOR TUNING}

To investigate whether despite the lack of chemotopy on the level of glomeruli, there might be chemotopic arrangements of MTCs, I analyzed NbT-positive and negative neurons from the calcium imaging experiments performed in NbT-Katushka animals the same way I did for the glomeruli and JGCs Similarity in odor response profiles (Pearson correlation coefficient) of NbT-positive neurons was independent of the neurons' somatic distance to each other (Figure 21, A). The tuning of NbT-negative neurons and their distance to each other was also found to be uncorrelated (Figure 21, B). However, there was a slight negative correlation for distance values above $150 \mu \mathrm{m}$. 
A

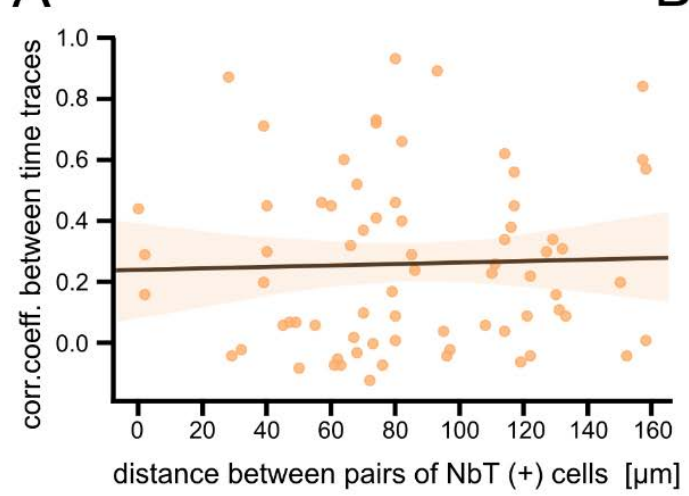

B

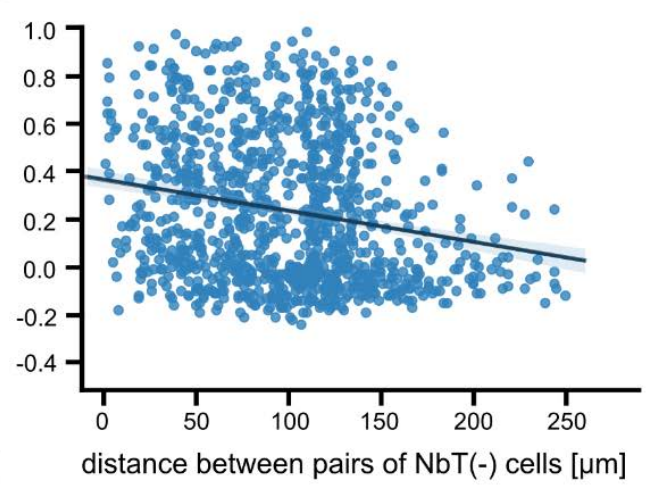

Figure 21 NbT-positive MTCs of similar odor tuning are not spatially clustered

A) Pooled data of NbT-positive MTCs (from 7 animals) with each dot (orange) representing the Euclidean distance and similarity in odor tuning (Person's correlation coefficient between fluorescence intensity time traces) between the two MTCs. No correlation between MTCs' position and odor tuning similarity was apparent (fitted line). B) No correlation between NbT-negative neurons' distances to each other and their odor tuning was apparent in the range of 10-150 $\mu \mathrm{m}$ distance. Slightly negative correlation might be present at higher distances (black line).

\subsection{SULFATED STEROIDS ARE SUITABLE STIMULI FOR THE MOB AND AOB NEURAL NETWORK OF LARVAL XENOPUS}

Prior to our study (Sansone et al., 2015), no suitable stimuli for the Xenopus AOS were known. Sansone could demonstrate in this study that $E$ and $P$ type sulfated steroids are suitable odorants of both the MOE and the VNO of larval Xenopus. I performed calcium imaging experiments in the larval Xenopus MOB (medioventral LC) and AOB to reveal whether sulfated steroids activate the postsynaptic neuronal network and glomeruli. I used a mix of amino acids as an additional positive control for the LC. I could observe extensive and strongly overlapping odor responses to $E$ and $P$ type sulfated steroid mixes in both the lateral MOB (Figure 22, A; right panel) and $A O B$ (Figure 22, A; right panel). In the MOB reactive regions (tufts of MTCs or MTC somata) were tuned differentially to sulfated steroids and amino acids (Figure 22, B and C). I found MTC somata that were more selectively tuned to one of the sulfated steroid mixes or to a combination of one of the mixes and amino acids (Figure 22, C; left panel). In contrast, in MTC somata of the AOB I did not measure any apparent responses to amino acids but only to the sulfated steroids on both the MTC tuft (Figure 22, B; lower panel) and somatic level (Figure 22, C; right panel). The reactive regions in $A O B$ and $M O B$ were very broad and consisted of both cell bodies and the postsynaptic glomerular neuropil. Upon transection of the ON, odorant responses to sulfated steroids were completely abolished (data not shown). 
A

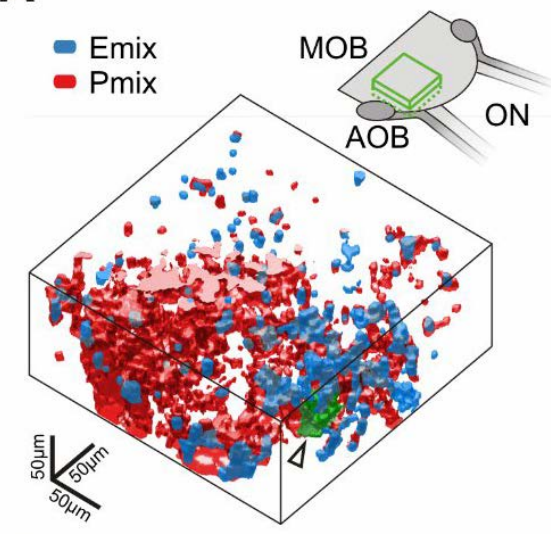

$\mathbf{C}_{\text {MOB }}$
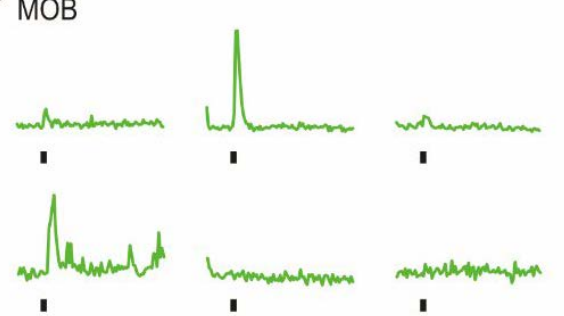

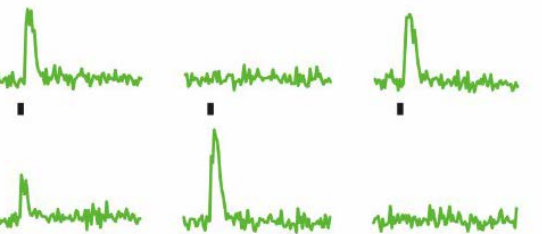
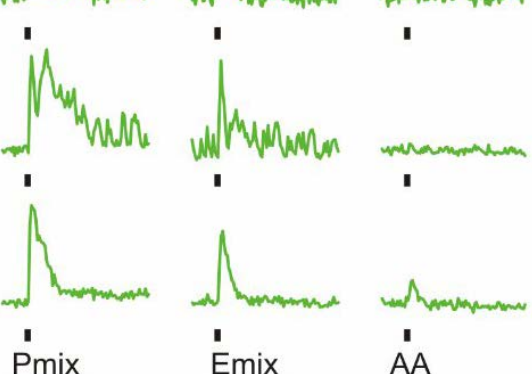
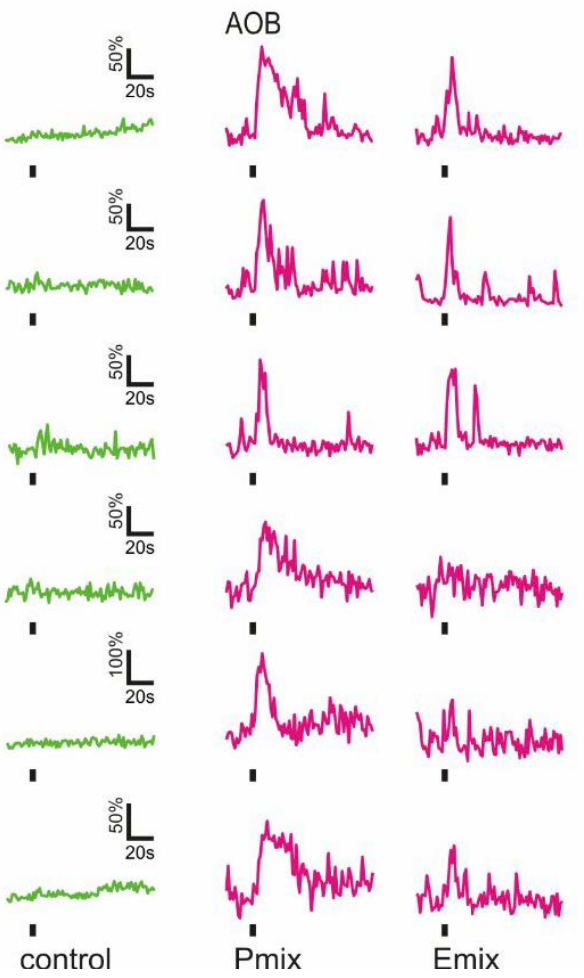

I ।
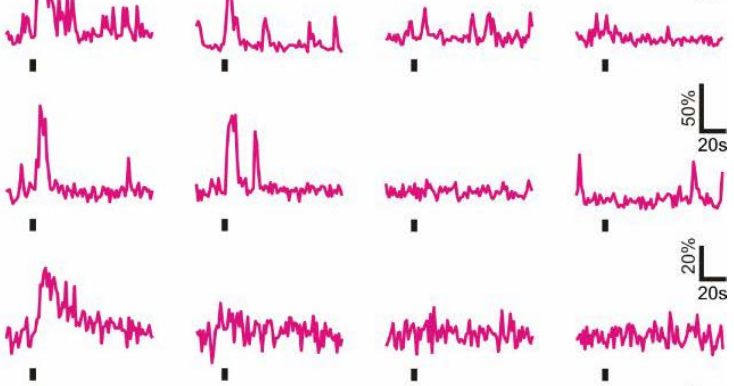

Nh/mimhan
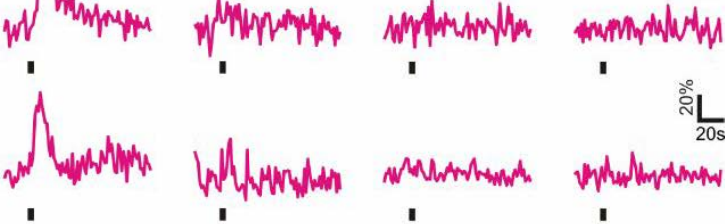

Mnth
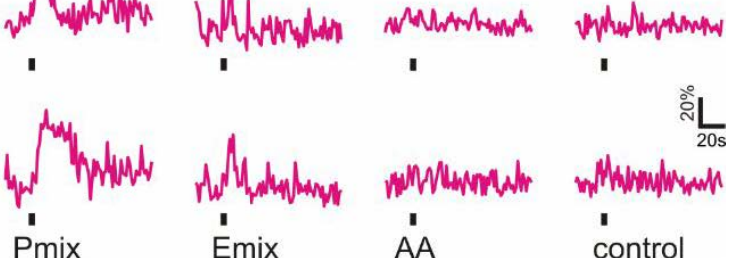

Figure 22 Sulfated steroids are processed in the olfactory bulb (Figure taken from Sansone et al., 2015, Figure 4; made by Thomas Hassenklöver; own published results)

(A) ' $3 \mathrm{D}$ rendering of reactive volumes in the $\mathrm{MOB}$ (left hand side) and $\mathrm{AOB}$ (right hand side) upon mucosal application of $E$ and $P$ mix (E mix-responsive areas, blue; $P$ mix-responsive areas, red). Active regions always contained both cell somata and glomerular structures. Schematics of the olfactory bulb in the upper part show the approximate location of the responsive regions. (B) Calcium responses of the glomerular regions indicated by an arrow and green color in $\mathrm{A}$ (MOB, green traces; $\mathrm{AOB}$, magenta traces). Amino acid mixture (AA, $100 \mu \mathrm{M})$ was applied as a positive control for activation of the MOB. (C) Representative calcium responses of mitral/tufted cells in the MOB (green) and AOB (magenta) upon mucosal application of sulfated steroids (E mix and P mix, $200 \mu \mathrm{M}$ ) and amino acids (AA, $100 \mu \mathrm{M}$ ). In both main and accessory olfactory bulb mitral/tufted cells were activated by either $\mathrm{E}$ or $\mathrm{P}$ steroids, or both stimuli. Amino acids only elicited calcium responses in mitral/tufted cells of the MOB. Similar results were obtained in 4 MOBs and 3 AOB.' (Figure legend taken literally from Sansone et al., 2015; Figure 4; own published results; written by Thomas Hassenklöver) 


\section{DISCUSSION}

\subsection{ZONAL DISTRIBUtion OF ORNs In MOE AND OB OF LARVAL XENOPUS:}

\section{The ventrolateral olfactory stream in larval Xenopus}

I used an adapted form of the WGA-based neuronal positioning system introduced by Tsuriel and colleagues to retrogradely label ORNs in the larval MOE (Tsuriel et al., 2015). I injected four WGA-coupled fluorophores of different colors into four distinct locations in the GL of the larval OB. The axon terminals subsequently took up different ratios of the individual tracer molecules via endocytosis (Tsuriel et al., 2015). I used the color identities of individual vesicular structures filled with tracer molecules in the ORNs' somata to deduce the vesicles' origin, i.e., the ORN's likely axonal target region in the OB. Vesicular structures in ORNs that matched the composition of tracer molecules in the LC could be found laterally enriched in the MOE of the larvae (Figure 1). This observation is in accordance with previous studies in the larval Xenopus olfactory system. Gliem and colleagues have proposed a lateral olfactory stream (Gliem et al., 2013): A laterally biased group of cAMP-independent, microvillous ORNs exhibits highly similar somatic odor tuning to amino acids, as the axon terminals associated with the LC. In the latter study, retrograde tracing of the lateral ON bundle was performed using biocytin electroporation (Gliem et al., 2013). My tracing experiments confirmed the partially segregated olfactory processing streams observed by Gliem and colleagues. I analyzed the entire sectioned MOE, scanning each tissue slice from both sides. This way, I could assess the mediolateral but also the dorsoventral dimension. In the ventral MOE, especially the region around the choane, there was a high density of vesicular structures matching in color composition with the LC. The signal in this most ventral part of the MOE was evenly distributed, with no apparent bias towards the medial or lateral side (Figure 1, F). These results highlight that the ORN population innervating the LC might be bigger and extend more ventrally than previously assumed. This region of the olfactory epithelium was most likely not addressed due to the different sample preparations used for calcium imaging or the biocytin tracing by Gliem and colleagues. The updated ventrolateral distribution of this ORN population in the larval MOE sheds light on a possible zonal organization of ORN distribution along the ventrolateral to dorsomedial axis, similar to rodents (Mori et al., 2000).

Possible roles of developmental timing and odorant receptors in instructing the zonal organization of larval Xenopus olfactory system

In the rodent MOS, a receptor subtype-dependent zonal patterning along the dorsoventral axis exists on both MOE and glomerular level, (Mori et al., 2000; Ressler et al., 1993; Vassar et al., 1993). The ventral glomerular domain is mostly targeted by OR type II ORNs (Mori et al., 2000; Takahashi et al., 2010) The dorsal domains receive axonal innervation from ORNs expressing OR type I or OR type II receptors (Mori et al., 2000; Tsuboi et al., 2006). This 
dorsoventral patterning is believed to be a partial result of developmental processes during olfactory map formation (Mori and Sakano, 2011). The timing of neurogenesis in different ORN species plays an important role in axonal navigation and hence, the sequence of glomerular formation (Eerdunfu et al., 2017; Mori and Sakano, 2011). In the rodent MOB, the glomerular map is sequentially formed along the dorsoventral axis, first starting with the most dorsal axonal projections. Later generated ORN species' axons use the latter as guidance and target the more ventral zones (Eerdunfu et al., 2017; Takahashi et al., 2010). In the radially-symmetric olfactory sensory surface of zebrafish, the timing of neurogenesis and radial migration shape the distribution of ORNs along the lamellae (Bayramli et al., 2017). It is not known in Xenopus laevis, whether the glomerular array forms sequentially. Ventral-to dorsal differences in receptor subfamily expression have also not yet been investigated. There is convincing evidence that the ORN population I traced in this work uses cAMPindependent signal transduction and to a certain extent, expresses ancestral V2R genes (Gliem et al., 2013; Syed et al., 2013). Second messengers like cAMP have been demonstrated to be essential in axonal guidance and instructing glomerular positioning in the MOB of rodents (Feinstein et al., 2004; Nakashima et al., 2013; Zou et al., 2007). Apart from external guidance cues and axon-axon interactions (Feinstein and Mombaerts, 2004; Mori and Sakano, 2011), the spontaneous activity of ORs in the axonal growth cone was shown to be detrimental in glomerular formation in the rodent MOS and linked to the cAMP level (Feinstein et al., 2004; Nakashima et al., 2013). The ventrolaterally-biased distribution of CAMP-independent ORNs in larval Xenopus might have fundamentally different axonal targeting mechanisms than the OR-expressing, cAMP-dependent ORNs. Differences in timing of neuronal differentiation and axonal outgrowth between the two ORN populations might be established early during development. Processes like axonal sorting and axonal growth cone guidance would then sharpen the segregation on the OB level, resulting in the distinct glomerular clusters observed (Gliem et al., 2013; Manzini et al., 2007a). Similar segregation exists in zebrafish, between their medial glomerular cluster and the lateral plexus (Braubach et al., 2012; Sato et al., 2005). Already early in development, the microvillous ORNs exclusively innervate distinct subsets of proto-glomeruli which will form the lateral plexus later on (Sato et al., 2005; Shao et al., 2017). Intriguingly, at that stage, co-expression of multiple odorant receptors of the respective receptor class was observed in individual ORNs (Shao et al., 2017). The expression of multiple receptors points to more general guidance mechanisms being responsible for the initial segregation of the two subsystems than receptor-based axonal navigation. In Xenopus laevis tadpoles, a narrowing in odor tuning profiles to amino acids was observed in the course of development between stages 50-51 to 52-54 (Manzini and Schild, 2004). Co-expression of several odorant receptors in ORNs at earlier developmental stages was proposed as one of the possible reasons for the developmental variability in odor tuning (Manzini and Schild, 2004). As in zebrafish (Shao et al., 2017), singular odorant receptor expression might not be pivotal for early axonal targeting 
in larval Xenopus. The olfactory system of Xenopus laevis originates from the olfactory placodes, which occur around developmental stage 23 (Klein and Graziadei, 1983). After the formation of the olfactory pits from the placodes the neuroepithelia undergo rapid cellular and morphological differentiation. During this phase, ORNs differentiate and display mature morphologies. However, it is only from stage 30 on that the ON forms and innervation of the OB begins. From stage 38 on the neuroepithelia closely resemble their mature versions (Klein and Graziadei, 1983). To find out whether the ventrolateral to dorsomedial zonal organization I observed in larval Xenopus is in fact a product of spatially distinct stem cell populations or timing in neurogenesis it would be necessary to investigate ORN differentiation between developmental stages 28 to 38 on both the MOE and the OB level. Suitable markers that label key elements of the two distinct olfactory streams will be crucial to get a clear insight into the fast, spatio-temporal processes taking place during early development.

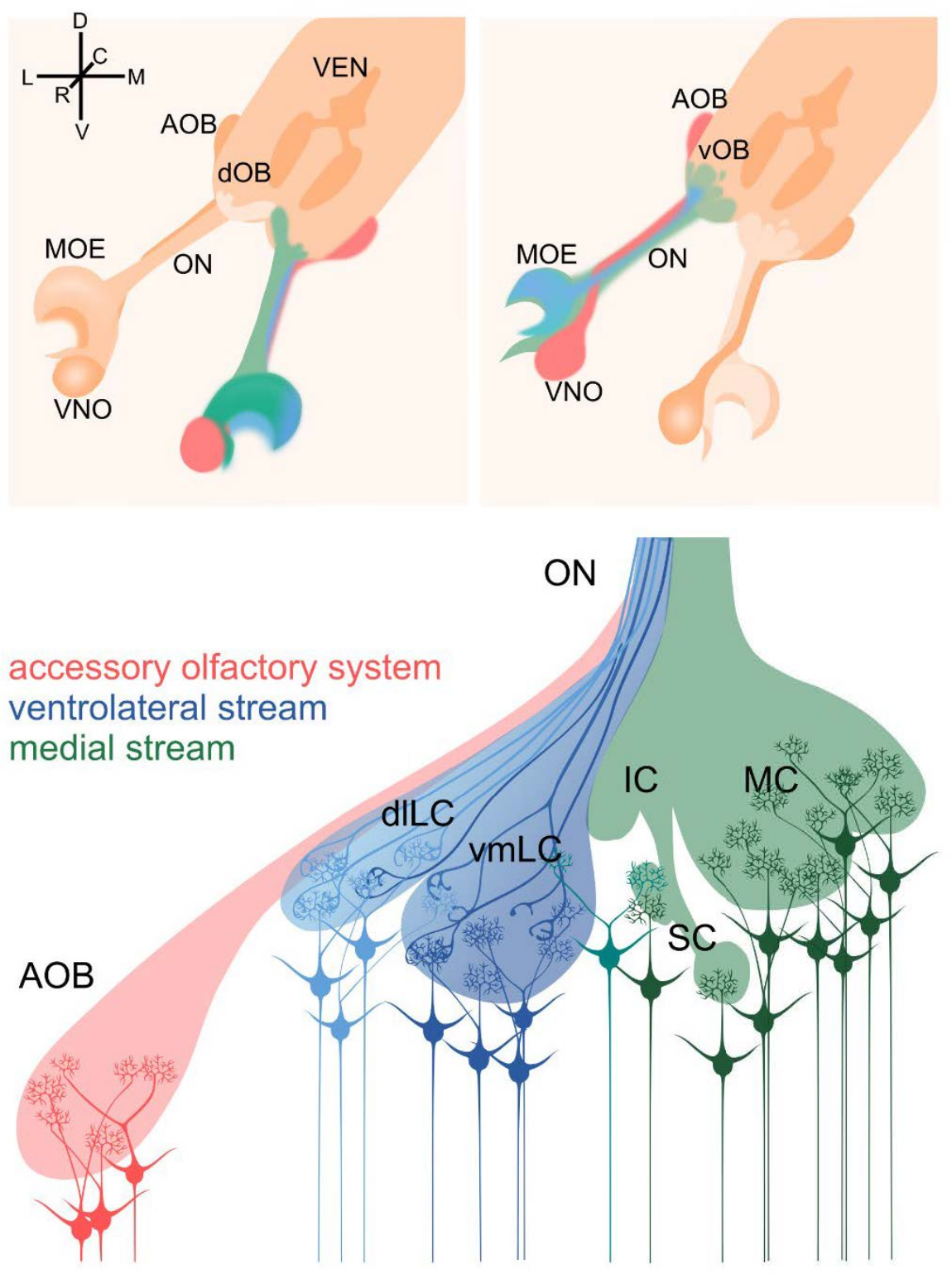

Schematic 17 The ventrolateral olfactory stream in larval Xenopus laevis and its subdivision on the glomerular and MTC level 


\section{Conserved segregation of olfactory processing streams on the level of MTCs - A result of MTC positioning or functionally distinct MTC populations?}

I performed multi-color Calcein AM dye injections to label subparts of the MTC population and to reveal their dendritic and axonal projections. The coarse division into glomerular clusters on the ORN level could also be observed on the MTC level. Somata of MTCs that projected their apical dendrites into the GL of the MC, IC, or LC were found clustered close to their respective glomerular clusters (Figure 2,B). However, I saw dendritic projections of individual MTCs beyond their most proximal cluster in both Calcein AM dye injection and sparse cell electroporation experiments. MTCs that clustered around the LC never projected their tufted dendrites as far as the MC and vice versa in this study. This coarse positional conservation of the odor processing streams on the MTC level is very likely a product of glomerular formation during development. While glomerular formation is a highly interactive process that involves both pre- and postsynaptic neuronal populations, the current view in the field is that ORN axon terminals have a major instructive role in glomerular formation which is in large parts independent of the postsynaptic network (Imai and Sakano, 2007; Imai et al., 2009; Mori and Sakano, 2011). Pre-existing postsynaptic target structures do not seem to be crucial for ORN axonal navigation during early glomerular development (Blanchart et al., 2006; Bulfone et al., 1998; Treloar et al., 1999). Very recent findings of Nishizumi and colleagues support the theory that MTCs form synapses with neighboring glomeruli in their proximity independent of receptor identity (Nishizumi et al., 2019). While the presynaptic glomerular scaffold seems mostly predetermined by the numerous ORN axonal guidance mechanisms (Imai and Sakano, 2007; Mori and Sakano, 2011), connectivity of MTCs to glomeruli is more dependent on the cells' positioning in the OB (Nishizumi et al., 2019). This hypothesis could explain the tight association of MTCs in Xenopus larval OB with their respective glomerular clusters, at least on the population level. The absence of MTC input integration from both the MC and LC could thus not only be due to the glomerular clusters' different signaling transduction pathways and odorant receptor types, but possibly due to the distance between them (Gliem et al., 2013; Manzini and Schild, 2003a; Manzini et al., 2007a).

I labeled subsets of MTCs associated with the LC to evaluate whether their somatic position was predictive of their primary dendritic projections among the glomerular cluster (See PRISMA technique in the methods section). I could observe a coarse topographic dependence between MTC soma positions and the positions of their tufted dendrites in the glomerular cluster (Figure 3; E, F, G and H). However, the projection fields were largely overlapping between the subpopulations investigated. Single tufted dendrites occasionally projected to non-proximal glomeruli all across the LC. The analysis of MTC morphology backs up those results: I found MTCs that had a proximal glomerular target but also others that innervated glomeruli scattered across the LC. The topological association of glomeruli and their neighboring MTCs as seen in rodents (Nishizumi et al., 2019) seems to also be present on the level of glomerular clusters in larval Xenopus. However, it needs to be taken into 
account, that Nishizumi and colleagues investigated MTCs in the MOS and thus mostly addressed the uni-glomerular MTC type (Nishizumi et al., 2019). The LC and maybe even the entire ventral bulb of larval Xenopus cannot be understood without taking the existence of multi-glomerular innervation patterns by individual MTCs into account (Nezlin and Schild, 2005); own results). The frequency and detailed features of those cells will be discussed in detail later on. The multiple tufted dendrites likely account for the less topological dendritic projections of the LC MTCs to a considerable extent.

\subsection{PARTIALLY SEGREGATED SUB-STREAMS OF THE LC BASED ON ODOR GROUPS AND VR EXPRESSION}

The partial segregation of the LC into ventromedial and dorsolateral lobe as a reflection of adjacent V2R and V1R expression domains

The injections of WGA-coupled fluorophores into the ventral OB strengthened the idea of the bimodal olfactory streams in larval Xenopus laevis (Gliem et al., 2013). Moreover, the staining revealed glomerular morphologies in a punctuate and less fiber-like manner than in dextran bulk electroporation (Figure 1; A). I described a dorsolateral lobe of the LC that is directly adjacent to the more prominent ventromedial lobe. The coarse boundaries between the lobes were distinguishable in stages 52-54. These two lobes are very likely the equivalent to the projection fields named PF4 and PF 5 by Gaudin and Gascuel (Gaudin and Gascuel, 2005). The segregation of the two projection fields / lobes were described, especially during ongoing development (Gaudin and Gascuel, 2005). I did not observe responses to amino acids in the dorsolateral lobe, at least within the limitations of the methods I used. While there is no concrete proof yet, ORN responses to amino acids in Xenopus laevis are highly correlated to V2R odorant receptor class expression (Syed et al., 2013, 2017). The retrogradely traced population of ORNs that innervate the two lobes of the LC might consist of both V2R and V1R-expressing neurons. One could speculate that the ventromedial lobe of the LC characterized in this work is dominated by V2R-expressing ORNs (amino-acid sensitive) while the dorsolateral lobe could be a target of V1R-expressing ORNs. In the rodent AOB, the two VR domains are dichotomous (Belluscio et al., 1999; Rodriguez et al., 1999). However, the individual neuronal networks processing the caudal and rostral domains' information interact on the bulb level (Larriva-Sahd, 2008). It might very well be that in larval Xenopus, two subsystems exist within the LC similar to the rodent AOB but less anatomically distinct. 


\section{Amino acids and sulfated steroids - Suitable odorants for different sets of V2Rs}

The calcium imaging experiments I performed in the LC of larval Xenopus MOB revealed that sulfated steroids induced odor responses on the glomerular and MTC level (Figure 22 published in (Sansone et al., 2015). Both, rodent V1Rs and V2Rs can potentially be activated by sulfated steroids (Hammen et al., 2014; Isogai et al., 2012). However, to the best of my knowledge, there are no reports of rodent V1Rs or ORAs in fish that are tuned to single amino acids. In rodents, a small but consistent population of sulfated steroid-responsive glomeruli was characterized in the posterior AOB domain (Hammen et al., 2014), which is assumed to be exclusively innervated by V2R-expressing VRNs (Belluscio et al., 1999; Rodriguez et al., 1999). The authors interpreted this surprising observation as either 'displaced' V1R glomeruli or a subset of $\mathrm{V} 2 \mathrm{Rs}$ that respond to sulfated steroids. Xenopus laevis possesses an extensive V2R repertoire, and V2Rs are broadly expressed in its MOE (Hagino-Yamagishi et al., 2004; Syed et al., 2013). Consequently, it seems relatively realistic that a set of V2Rs could carry sensitivity to sulfated steroids in Xenopus. Different contributions of V1Rs and V2Rs might explain differences in detection thresholds of sulfated steroids between the VNO and MOE (Sansone et al., 2015) could be explained by different contributions of V1Rs and V2Rs. The AOS of Xenopus laevis harbors phylogenetically younger, mammalian-like V2Rs and lacks the more ancestral 'fish-like' V2Rs (Hagino-Yamagishi et al., 2004; Syed et al., 2013). The glomeruli and AMCs I measured in larval Xenopus AOB, did not respond to amino acids, but to both sulfated steroid mixes (E- and P-mix). If one assumes these response profiles to be representative for sets of later-diverging V2Rs of the AOS, the glomerular responses to sulfated steroids in the larval MOS are more likely a result of the ancestral V2Rs or V1Rs expressed (Date-Ito et al., 2008; Hagino-Yamagishi et al., 2004; Syed et al., 2013).

There is evidence from in situ hybridization studies, that Xenopus V2Rs and the V2R-C receptor could selectively be co-expressed (Syed et al., 2013) similar as observed in fish (DeMaria et al., 2013) and rodent V2Rs (Ishii and Mombaerts, 2011; Silvotti et al., 2007). In zebrafish, the OlfCc1 receptor serves as amino acid sensor and co-receptor for other OlfC receptors. Loss of OlfCc1 results in general loss of sensitivity to polar, basic, and acidic amino acids (DeMaria et al., 2013) and thus might be essential to detect food stimuli in zebrafish (Koide et al., 2009). Amino acid responses in the ventromedial lobe could be explained by similar combinations of Xenopus ancestral V2Rs and the broadly expressed v2r-C receptors (Syed et al., 2013). V2Rs tuned to hydrophobic, hetero-cyclic features in amino acid residues like tryptophan might potentially also be activated by similar features found in the sulfated steroids as a result of combinatorial receptor coding (Malnic et al., 1999; Sansone et al., 2015).

The calcium imaging experiments in the LC of the larval MOB revealed MTCs exclusively responding to either sulfated steroids or amino acids but also subsets that responded to both groups (Figure 22). Amino acid-insensitive glomeruli that reacted to sulfated steroids might 
carry information from V2Rs tuned to particular features of sulfated steroids that are not present in amino acids. Another possibility is that the amino acid-insensitive fraction of sulfated steroid-responsive glomeruli in Xenopus receives input from V1Rs. In rodents, V1Rs can be activated by sulfated steroids already at concentrations of $1 \mu \mathrm{M}$ (Hammen et al., 2014). The apparent sensitivity of the larval Xenopus MOS to sulfated steroids could be composed of a more sensitive V1R part and a less sensitive V2R part (Sansone et al., 2015). The V2Rbased responses to sulfated steroids at higher odorant concentrations (Sansone et al., 2015) could be due to less specific odorant receptor-binding This could explain the lower average sensitivity of ORNs in the MOE to sulfated steroids (both V1R and V2R components) in comparison to the VRNs (V2R components only; (Date-Ito et al., 2008; Sansone et al., 2015). If the dorsolateral lobe (PF5) should indeed represent the amino acid-insensitive V1R expression zone, its small size could be a reflection of the low number of V1R genes in Xenopus laevis (Date-Ito et al., 2008; Shi and Zhang, 2007). However, quantification of this observation will be necessary. To sum it up, the described ventromedial lobe of the LC could represent a non-exclusive V2R expression domain with amino acids and sulfated steroids as potent stimuli. Its responsiveness to amino acids might be based on ORNs co-expressing ancestral V2Rs and V2R-Cs. The responses to sulfated steroids in Xenopus MOB could be a result of low-affinity binding to those ORNs' odorant receptors at high odorant concentrations. Alternatively, V2R-expressing ORN species tuned to sulfated steroids might account for the observed responses. The dorsolateral lobe might represent the amino-acid insensitive V1R expression zone, possibly responsive to sulfated steroids. No evidence for cAMP-dependent olfactory signal transduction was found in the LC of larval Xenopus (Gliem et al., 2013). The $G$-protein $G_{i_{2}}$ seems to be associated with the signal transduction cascade of V1Rs in Xenopus (Date-Ito et al., 2008). Immunohistochemical stainings against $\mathrm{G}_{\mathrm{i}_{2}}$ or calcium imaging using V1R specific ligands would undoubtedly help to deduce odorant receptor contributions to the observed ventromedial and dorsolateral lobe. Overall, the first part of my results confirms but at the same time, expands the existing idea of the bimodal olfactory processing streams in larval Xenopus laevis. I could reveal the full extent of the ventrolateral ORN population projecting to the LC. MTCs associated with particular glomerular clusters reside in proximity to those. Their somatic position can be predictive for their dendritic glomerular innervation among the LC. However, there are subsets of MTCs that can project to distal or even multiple glomeruli scattered across the glomerular array. In addition to amino acids, I established sulfated steroids as suitable stimuli for the postsynaptic neuronal network associated with the LC. The observed glomerular response patterns to those odorant groups, rule in the possibility of two distinct, but partially overlapping subsystems within the LC. The medioventral and dorsolateral lobes of the LC could be reflections of different VR-based subsystems 


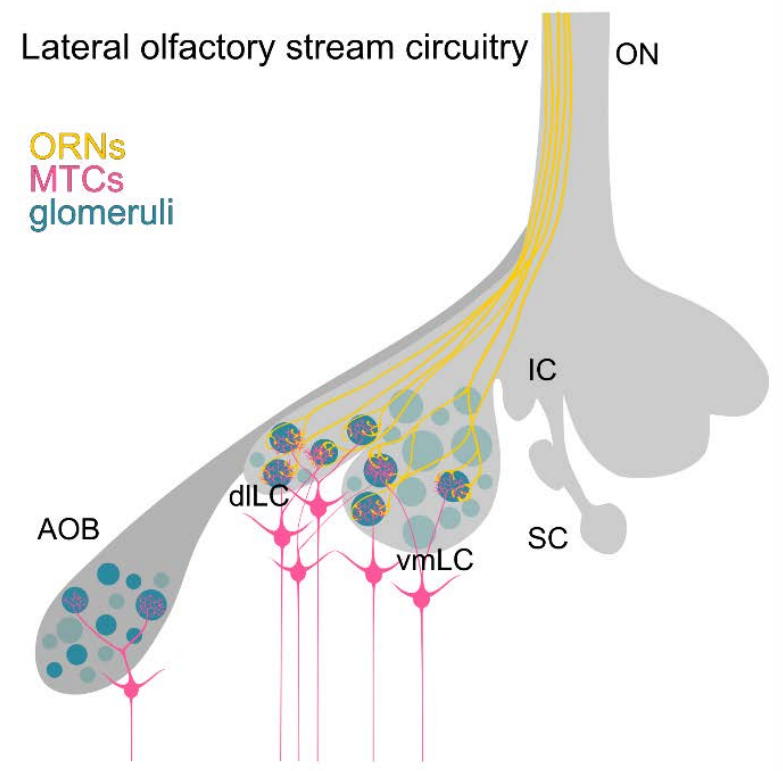

Glomerular odorant responses

sulfated steroids (V2R) sulfated steroids (V1R) amino acids (AA)

sulfated steroids + AA

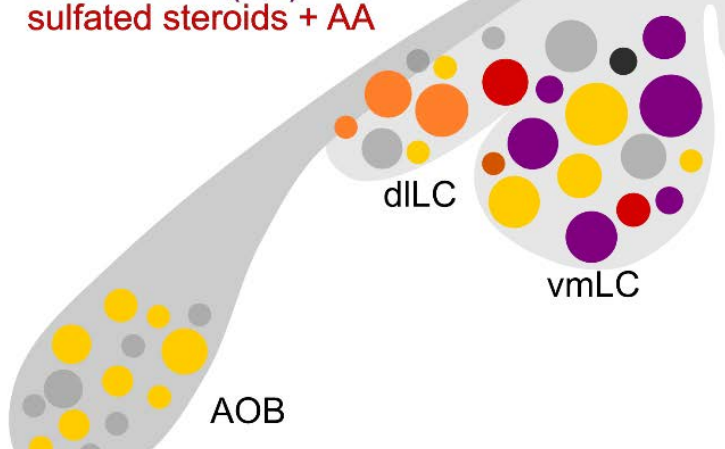

Schematic 18 Distribution of glomerular reactivity to different odorant groups among the $L C$ lobes and the $A O B$

\subsection{ODOR MAP ORGANIZATION OF THE LATERAL GLOMERULAR CLUSTER Lack of chemotopy as a major feature of the lateral olfactory system?}

Calcium imaging in the OB network of the $\mathrm{LC}$ revealed that there seems to be no stereotypical glomerular odor map in larval Xenopus. At least there is no evidence for consistency in glomerular position or number between bulbs of different animals (Figure 18). These results were somewhat surprising, especially since the zebrafish $\mathrm{OB}$ shares many similarities with the one of larval Xenopus. Both possess a lateral cAMP-independent amino acid-sensitive olfactory stream that is anatomically and functionally distinct from the medial CAMP- and ORdependent stream (Gliem et al., 2013; Sato et al., 2005). Using single amino acids as stimuli, Friedrich and colleagues could demonstrate that a coarsely chemotopic and stereotypical glomerular odor map exists in the lateral OB of zebrafish (Friedrich and Korsching, 1997). In the following paragraphs, I will discuss several possibilities for this discrepancy in the two seemingly similar aquatic systems.

Variable uptake of calcium indicator by MTCs might lead to heterogeneity in odor map

One of the caveats of the MCBL method I used is that variability in injection might lead to heterogeneous odor responses. This variable labeling of MTCs could distort the number and distribution of reactive glomeruli. In the experiments using the PRISMA method, Calcein AM dyes were injected into the MCL of the LC, the same way as Fluo-4 AM. Calcein AM dye uptake leads to increased and confined fluorescence in healthy, living cells, highlighting their morphologies (Chan et al., 2012). While undoubted, not every single cell in the MCL was labeled, the postsynaptic neuropil was densely stained (Figure 3, E). Even if individual MTCs had not taken up Fluo-4 AM dye, a sufficient number of odor-responsive MTC tufted dendrites should have been observable. Despite the variable number of glomeruli I observed between 
animals (Figure 18), coarse molecular feature clusters of glomeruli as seen in zebrafish (Friedrich and Korsching, 1997) and rodents (Matsumoto et al., 2010; Uchida et al., 2000) should have been noticeable if the larval LC was similar to the rodent MOB.

\section{Possible discrepancies between presynaptic and postsynaptic glomerular odor representation}

Most early functional mapping experiments in zebrafish or rodents were performed on the ORN glomerular level or derived from imaging of changes in intrinsic signal (Bozza et al., 2004; Friedrich and Korsching, 1997; Matsumoto et al., 2010; Uchida et al., 2000). The presynaptic glomerular activity is highly synchronized on the temporal scale. Its homogeneity amplifies and enhances the ORN species' input information (Wachowiak et al., 2004). While calcium signals recorded from the presynaptic side of glomeruli might be stereotypical, there is the possibility that the postsynaptic odor map could be different. Possible mechanisms might involve modulation or inhibition at the level of synaptic transmission by the local interneurons (Lizbinski and Dacks, 2018). At least in the rodent MOS, this does not seem to be the case. To the best of my knowledge, Storace and Cohen were the first to compare glomerular odorant responses on both the pre- and postsynaptic side in rodents. They simultaneously measured glomerular responses from a green genetically-encoded voltageindicator expressed in the postsynaptic network and a red calcium indicator in the presynaptic ORN axons. Their results pointed out that the postsynaptic glomerular odor map was actually less variable than the presynaptic side and robust across several odor concentrations (Storace and Cohen, 2017). In both zebrafish and mice, an increase of odor concentration leads to the recruitment of bigger sets of glomeruli. This effect is mainly attributed to the combinatorial receptor coding (Fried et al., 2002; Malnic et al., 1999; Meister and Bonhoeffer, 2001; Storace and Cohen, 2017). I used $100 \mu \mathrm{M}$ single amino acid solutions as odor stimuli and applied them for 5 seconds. At this concentration and stimulus duration, a maximal set of glomeruli should theoretically be activated (Manzini et al., 2007b). Auto-excitation (Salin et al., 2000) and glutamate spill-over at the MTC tufts (Isaacson, 1999)should additionally amplify the presynaptic signal and consequently contribute to the robustness of the postsynaptic odor representation (Schoppa and Westbrook, 2001; Storace and Cohen, 2017). Simultaneous calcium imaging on both the pre- and postsynaptic level would be crucial to confirm the congruence of both glomerular odor representations in larval Xenopus.

\subsection{ODOR REPRESENTATION IN A DEVELOPING OLFACTORY SYSTEM}

(When) is the lateral olfactory system fully developed?

One of the first things that come to mind when discussing reasons for the heterogeneity of the odor map in our larval animal model is, of course, development itself. The olfactory system of Xenopus laevis is influenced even stronger by developmental processes than that of many fish. In addition to the embryonic development of the larval olfactory system, their olfactory 
system undergoes massive restructuring during metamorphosis, including the formation of a new subsystem for air-borne odor detection (Altner, 1962; Dittrich et al., 2016; Hansen et al., 1998). In the pro-metamorphic stages I used in my work (Stages 52-54), a functional aquatic olfactory system should exist already. Unpublished behavioral data of our group could validate that tadpoles from stage 51 on show attraction to amino acids i.e., behavioral responses to known stimuli of the ventrolateral olfactory system (Hawkins et al., unpublished data). Amino acids were described as suitable stimuli in other aquatic animals like zebrafish, where they can serve as cues for the presence of food (Braubach et al., 2009; Koide et al., 2009). However, there is no direct evidence so far that behavioral responses to amino acids of larval Xenopus are related to food stimuli. Two observations by Hassenklöver and colleagues challenge the food stimulus character of amino acids in larval Xenopus: First, ORNs are preferentially tuned to single amino acids instead of the more common peptide derivates from protein degradation (Hassenklöver et al., 2012). Second, to elicit strong odorant responses, concentrations of amino acids in the micromolar range were necessary (Manzini and Schild, 2004; Manzini et al., 2002; Schild and Manzini, 2004). Amino acid concentrations in that range do not occur naturally in estuarine water bodies (Coffin, 1989; Jørgens, 1986). In this light, the diverse but at the same time selective odor tuning profiles to amino acids I recorded in glomeruli and MTCs seem even more puzzling. A possible explanation for these seemingly contradictory observations could be that the source of single amino acids detected by the olfactory system of larval Xenopus might not be the surrounding water body. Pipids like Xenopus laevis do not possess ciliated palates to transport particles towards the esophagus actively. However, ciliated grooves exist in the ventral part of the choane that can actively transport mucous and particles from the nostrils to the larynx of the larvae (LeCluyse et al., 1985). Xenopus tadpoles are water-dwelling filter-feeders that use continuous buccal pumping for respiration and feeding (Ryerson and Deban, 2010; Seale et al., 1982). The olfactory organs of fish are often adapted to direct the laminar flow of odorants along the lamellae during swimming or create vortices by motile cilia to optimize odor detection (Cox, 2008). It is imaginable that buccal pumping, motile cilia, or a combination of both deliver fractions of amino acid-enriched water from inside the animal's larynx through the choane towards the olfactory mucosa.

The concentration of amino acids in these fractions might be higher than in the surrounding water and lead to the activation of ORNs. Retro-nasal olfaction plays an essential role in mammalian olfaction and is tightly-linked to breathing rhythms (Gautam and Verhagen, 2012; Rebello et al., 2015). This hypothesis of 'retro-nasal' amino acid-detection in Xenopus tadpoles could also explain the ventrolateral enrichment of amino acid- sensitive ORNs I observed from a 'hydrodynamic point of view.' The lateral olfactory stream might serve the mostly stationary filter-feeders (Seale et al., 1982) to asses information about ingested material in their surroundings, rather than to provide positional information about (distant) food sources as in zebrafish (Koide et al., 2009). It is imaginable that the lateral glomerular 
map is non-stereotypical because it is still undergoing developmental processes. Despite the ongoing developmental processes, it might suffice for its task to analyze the ingested food via the ventrolateral ORN population close to the choane. The active location of odor sources (Catania, 2013; Rajan, 2006) might be a fundamental driving force that has shaped the glomerular map organization in certain mammalian MOBs. Such an odor map might thus be absent in tadpoles since active searching of odor sources might not be crucial to the tadpole's survival.

\section{Lack of stereotypy in a developing, partially 'proto-glomerular 'map}

The glomerular array of pro-metamorphic tadpoles could partially consist of proto-glomeruli as in developing zebrafish (Shao et al., 2017). Proto-glomeruli are innervated in a receptor class-exclusive manner but can be composed of different ORN species (Shao et al., 2017). During maturation of ORNs, a single odorant receptor is chosen, which predetermines its target proto-glomerulus (Shao et al., 2017). Mature glomeruli only emerge later by compartmentalization of the proto-glomeruli through a couple of glomerular refinement processes that were mainly discovered in rodents and will not be addressed here in detail (Feinstein and Mombaerts, 2004; Hasegawa et al., 2012; Mori and Sakano, 2011). The variability in glomerular position, size, and number I observed in my calcium imaging experiments might be a postsynaptic reflection of dynamic glomerular refinement. A narrowing of odor response tuning in ORNs of larval Xenopus was observed between early (51-53) and late (54-56) pro-metamorphic stages (Manzini and Schild, 2004). Both more and less selectively tuned ORNs could thus contribute to the glomerular ensemble of the LC in the form of mature or proto-glomeruli. The expression of multiple odorant receptors in individual (developing) ORNs has been discussed in Xenopus and might contribute to the diversity in odorant response profiles of ORNs and glomeruli (Schild and Manzini, 2004; Manzini et al., 2007). In contrast to other vertebrates investigated (Dynes and Ngai, 1998; Klenoff and Greer, 1998; Mombaerts et al., 1996), ORN axons of larval and adult Xenopus laevis bifurcate and can innervate one or several (mostly two) distinct glomeruli (Hassenklöver and Manzini, 2013; Nezlin and Schild, 2005). This feature could increase the variability of proto-glomerular pruning and contribute to the larval odor map's heterogeneity.

\section{Lack of stereotypy due to 'metamorphosis of odor representations' in the larval aquatic OB}

Another reason for the heterogeneous lateral glomerular map could be the onset of metamorphic remodeling. During metamorphosis, the principal cavity and former larval waternose is completely remodeled into an air-nose that will innervate the emerging dorsal $O B$. Simultaneously from stage 52-53 on, the middle cavity emerges in the periphery and starts innervating the ventral OB forming the new aquatic olfactory system (Altner, 1962; Dittrich et al., 2016; Hansen et al., 1998). At these stages, ORN axons from the middle cavity start innervating the ventral $\mathrm{OB}$, whereas ORN axons from the principal cavity are eliminated by 
apoptosis in the process of transformation into an air-nose (Dittrich et al., 2016). This turnover might also lead to variability in the glomerular composition and distribution. However, the peak of these processes should happen in later metamorphic stages from stage 56 on (Dittrich et al., 2016). Developmental effects might account for numerous features observed in the heterogeneous glomerular map of larval Xenopus. However, the possibility needs to be ruled in, that the glomerular odor map of the LC does not follow the canonical axonal wiring paradigm of the MOB in rodents (Mombaerts, 2006; Mombaerts et al., 1996). On the contrary, my investigations of the lateral olfactory stream strongly suggest, that the glomerular map and wiring logic in larval Xenopus laevis has more similarities to an AOS-like configuration.

\section{The lateral glomerular map in Xenopus laevis: An AOS-like odor representation}

Single amino acids are powerful stimuli to map chemotopy in aquatic olfactory systems. They share certain structural features like the amine and carboxy-group, but at the same time, offer a wide range of different molecular epitopes in their defining residue (Friedrich and Korsching, 1997). The coarse chemotopic arrangement of glomeruli in the lateral OB of zebrafish (Friedrich and Korsching, 1997) had similarities with the molecular feature clusters in rodents (Matsumoto et al., 2010; Uchida et al., 2000). I did not find any evidence for consistent molecular feature clusters in the LC of larval Xenopus. Despite the existence of recurrent glomerular response profiles, the distribution of similarly tuned glomeruli was very heterogeneous and inconsistent. This inconsistency was very surprising, especially since the main groups of odor response profiles I derived from hierarchical cluster analysis, were virtually identical to the ones observed in zebrafish (Friedrich and Korsching, 1997). They also clustered according to their amino acid residues' structural similarity, as seen in zebrafish (Friedrich and Korsching, 1997). In addition to the lack of stereotypy, I could show that glomeruli with dissimilar odor tuning often appeared adjacent to each other (Figure 19). This juxtaposition of glomeruli with uncorrelated or even negatively correlated MRR is a key feature of the glomerular odor representation observed in rodent AOB (Hammen et al., 2014). Pair-wise comparison between larval Xenopus glomeruli revealed that there was no spatial clustering of similarly tuned glomeruli. The lack of stereotypy and a consistent odor map is another characteristic feature seen on the VRN level in the AOS of rodents (Belluscio et al., 1999; Rodriguez et al., 1999). In mammalian systems, there is high variability in number and positioning of glomeruli formed by an individual VRN species (expressing one VR-type receptor). This variability can not only be observed between different animals but also between the two bulbs of the same animal (Belluscio et al., 1999; Rodriguez et al., 1999). In a pioneering study performed by Wagner and colleagues, three V1R-expressing VRN species were fluorescently labeled in the same transgenic animal to investigate the glomerular distribution in the rodent AOB (Wagner et al., 2006). It turns out that the distribution of glomeruli in the anterior $\mathrm{AOB}$ is not random, but organized in a grid-like manner: Glomeruli innervated by VRNs that express closely related V1Rs (in this case V1Ra1 and 
V1Ra3 from the subfamily a) were found dispersed but intermingled on a particular level on the dorsoventral axis. V1Rb1 (subfamily b) expressing VRNs formed glomeruli on a more dorsal level of the 2D grid (Wagner et al., 2006). Glomeruli of the same V1R identity dispersed along the anterior-posterior axis of their particular dorsoventral grid level. Glomeruli innervated by V1Rs of different subgroups were found in a fixed distance to each other along the dorsoventral axis of the AOB (Wagner et al., 2006). I could not find a similar 2D grid-like pattern in the distribution of amino acid-sensitive glomeruli in the LC, at least when categorizing them by their dominant odor tuning properties. However, this does not mean that there is no geometrical scaffold along which glomeruli are distributed. The 3D-structure of the lateral glomerular cluster in Xenopus (Manzini et al., 2007a) cannot be as easily divided into a grid as in the AOB of rodents (Wagner et al., 2006). It is very well possible that axonal guidance in such a three-dimensional setting still follows similar mechanisms of glomerular distribution as described in rodents by Wagner and colleagues. I investigated the glomerular distribution in a maximum projection with the x-coordinates being the ventrolateral and the $y$ coordinates being the anterior-posterior axis. The observed geometry of glomerular distribution cannot be easily understood nor quantified from the perspective I chose. Even more importantly, one of the major hallmarks of the proposed grid model is that the similarity between amino acid sequences of the V1Rs defines whether there is spatial clustering (Wagner et al., 2006). This is in contrast to the spatial clustering of glomeruli by molecular features of their ligands, as it is the case in the MOB (Mori et al., 2006). Overall sequence similarity between receptors is just coarsely predictive for the similarity in their molecular receptive range (Wagner et al., 2006). Since amino acids exhibit both diverse but at the same time similar molecular features (Friedrich and Korsching, 1997), it could very well be, that spatially clustered glomeruli of weakly or even uncorrelated MRR are formed by VRNs expressing phylogenetically related receptor variants (Hammen et al., 2014; Wagner et al., 2006). That means that receptor-based patterned distributions among the glomerular array would not be easily visible by just looking at odor tuning.

It is tempting to extrapolate the principles of glomerular distribution found in the V1Rexpressing domain (Wagner et al., 2006) to the entire AOB. At the moment, it is unknown whether the same organizational principles apply to the V2R-expressing domain in the posterior AOB in rodents (Del Punta et al., 2002; Rodriguez et al., 1999). As highlighted in previous paragraphs, ancestral V2Rs and their class C co-receptors are the most promising candidates for amino acid sensitivity in Xenopus laevis lateral olfactory stream (Gliem et al., 2013; Syed et al., 2013). The glomerular distribution I observed might thus follow different V2R-dependent axon guidance mechanisms. Del Punta and colleagues investigated the glomeruli formed by V2r1b expressing VRNs in the rodent AOB. Six to ten glomeruli were innervated by a VRN species. The glomeruli were dispersed among restricted areas of the caudal AOB (Del Punta et al., 2002). The relative 'depth' of V2r1b expressing glomeruli in the rodent GL was very comparable and seemed to be a characteristic parameter of the 
glomerular ensemble (Del Punta et al., 2002). Larval Xenopus possesses a laminar organization of the main histological layers, namely nerve-layer, GL, MCL and GCL. However, the GL of the LC consists of 'grape-like' groups of unparceled glomeruli, which makes the analysis of their relative positioning difficult (Nezlin and Schild, 2000).

\section{Sensory experience and its impact on glomerular map variability}

The unparceled and seemingly variable character of the LC in larval Xenopus could also be partially explained by an intriguing phenomenon of glomerular formation observed in zebrafish. Braubach and colleagues mapped the distribution of glomeruli in zebrafish using immunohistochemistry against a plethora of molecular markers. They could identify around 150 glomeruli in adult zebrafish, of which 27 were unambiguously identifiable between animals (Braubach et al., 2012). In a follow-up study, they analyzed the sizes and numbers of the smaller, less distinct glomeruli that also frequently occurred in the unparceled lateral OB (Braubach et al., 2012, 2013). They investigated numbers and sizes of glomeruli between animals that were reared in an environment enriched with amino acids and animals that were raised without this additional enrichment during their larval stages. Glomeruli were found to be more numerous and smaller in diameter in animals that experienced environmental enrichment during development in comparison to the control group (Braubach et al., 2013). The impact of this sensory experience on the development of larger stereotypic glomeruli was also assessed: It turned out, that this set of glomeruli formed already before hatching and stayed virtually invariant for both conditions (Braubach et al., 2013). While our tadpoles were all raised under controlled conditions in the animal facility, it could be that the variability in the amino acid-sensitive glomerular map is also a product of different sensory experiences among animals. A lack of biologically relevant olfactory stimuli of their wildlife habitat (Woodley, 2014) could lead to the observed variability in glomerular size and positioning. It is due to the encountered difficulties in distinguishing individual glomeruli of the LC that performing a similar experiment with larval Xenopus would be challenging. However, my protocols of labeling glomeruli in living tadpoles via WGA-coupled dextrans might still offer the best shot at making glomerular boundaries distinguishable at the moment.

Sensory experience and its impact on cellular composition and connectivity are also reported in the AOS of rodents: Mice that underwent deprivation of olfactory sensory input were shown to exhibit differences in sizes of VRN populations or even the absence of individual VRN species compared to control animals ( $\mathrm{Xu}$ et al., 2016). Most strikingly, some sexual dimorphisms on the VRN level that were observed in this study were dependent on exposure to scents of the other gender rather than solely induced by internal hormones (Xu et al., 2016). These differences in VRN populations found in the latter study should impact the glomerular configuration in the rodent $A O B$ as well. In my work, I did not distinguish between female or male larval tadpoles. Both sensory experiences, as well as effects of sexual dimorphism, could additionally contribute to the observed heterogeneity of glomerular maps. If the lateral 
olfactory stream was indeed a more AOS-like system, as I am proposing in this work, the variability in glomerular odor representation could actually be an essential feature of this subsystem. It would enable animals already in larval stages to adapt their olfactory circuitry to environmental or social demands. It should be noted that the glomerular variability by sensory experience observed in zebrafish might underly completely different mechanisms on the circuit level (Braubach et al., 2013). Zebrafish MTC primary dendrites almost exclusively form individual tufts projecting to one glomerulus (Fuller et al., 2006; Wanner et al., 2016). In the rodent $A O B$, the majority of $A M C s$ project multiple tufted dendrites to numerous olfactory glomeruli (Larriva-Sahd, 2008; Takami and Graziadei, 1991). In the next paragraphs, I will discuss how the morphological subtypes of MTCs I characterized in the lateral olfactory stream of Xenopus might further support the hypothesis of rodent AOS-like features in this subsystem.

\subsection{MTCS IN LARVAL XenOPUS LAEVIS - A MORPHOLOGically HETEROGENEOUS GROUP OF NEURONS} Identification of MTCs and methodological limitations

To characterize the morphology of MTCs, I established the method of sparse-cell electroporation in the MCL larval $\mathrm{OB}$. The technique is based on the single-cell electroporation method (Haas et al., 2001), especially in the peripheral olfactory organs of Xenopus laevis (Hassenklöver and Manzini, 2013; Weiss et al., 2018). While the method turned out to be an elegant and fast way to collect individual cells' morphologies, some methodological caveats need to be discussed beforehand.

I used two different approaches to target the $\mathrm{MCL}$ in the olfactory explant of tadpoles specifically: The first approach was to perform bulk electroporation with dextran-coupled fluorophores of the MOE two to three days before the experiment. Electroporation with a different fluorophore-coupled dextran allowed me to visualize the glomerular clusters and thereby have a better orientation, where the MCL was located (described in detail in Weiss et al., 2018). Additionally, I used animals of a Xenopus laevis transgenic reporter line in which the deep-red fluorescent protein Katushka was expressed under the $3.8 \mathrm{~kb}$ Xenopus neuronal beta-tubulin (NbT) promotor (Moody et al., 1996); created by the EXRC see methods). My investigations revealed that the NbT promotor labels ORNs and a subset of cells in the histological MCL. I tried to target the fluorescent somata of those NbT-positive cells directly. However, due to the dense, surrounding dendritic meshwork from other neurons, it was often the case that the electroporation labeled additional cells than the one targeted. I had to use fluorescent dextrans of different spectral properties than the expressed Katushka protein. Moreover, high concentrations of the tracers $(3 \mathrm{mM})$ were necessary to visualize the morphological details of MTCs in multiphoton-microscopy. The resulting spectral bleedthrough of green and orange dextrans into the far-red emission channel was so high that 
Katushka fluorescence could not be unambiguously confirmed anymore in the electroporated samples. This methodological caveat made it difficult to ascertain that all visible neurons after electroporation were NbT-positive. However, it can be stated that in none of the experiments, I targeted NbT-positive cells, any other cell type than MTCs were labeled. These results strongly suggest that NbT-positive cells are (mostly) MTCs. There was also a significant number of neurons in the MCL that did not show NbT fluorescence (See also calcium imaging results of NbT animals). It would need a fluorescent $\mathrm{NbT}$ reporter line in which the fluorescent protein expressed emits fluorescence of lower wavelengths than the dextrans used for targeted electroporation. That way, spectral bleed-through even at high tracer concentrations would be negligible, enabling a clear distinction of NbT-positive from accidentally labeled neurons. Expression of the fluorescent protein Katushka nicely delineated the boundaries of the MCL in larval Xenopus. Consequently, sparse-cell electroporation in the delineated $\mathrm{MCL}$ was possible with multiple colors, increasing the yield of labeled MTCs.

The electroporation of MTC somata often led to their death and subsequent neuritedegradation. This cellular degradation in the explant made it particularly challenging to distinguish the axon from possible basal secondary dendrites in MTCs. Moreover, in some cases, it was not easy to tell whether the neurites ended in the OB or the dye did not travel farther. In this work, I will discuss both axons and basal secondary dendrites as basal neurites, to not draw wrong conclusions about MTCs basal dendrites or axonal projection targets. If only one thin neurite emerged from the MTC soma and projected towards the caudal part of the $\mathrm{OB}$, it was reasonable to assume that this neurite represented an axon. In the end, MTCs/AMCs are the primary, axon-bearing output neurons of the MOB/AOB (Igarashi et al., 2012; Larriva-Sahd, 2008)

\section{MTC morphological subtypes - Do multiple tufts imply multi-glomerular wiring.}

MTCs of the larval lateral olfactory stream were very heterogeneous in shape and dendritic configurations. I reconstructed 77 MTCs and classified them by their basic morphological features. I subdivided MTCs by the number of distinct primary dendritic tufts: There were MTCs that did not bear any apparent tufts (5\%), MTCs with apical dendrites converging to one tuft (31\%) and MTCs with multiple, anatomically distinct dendritic tufts ( $\left.{ }^{*} 42 \%\right)$. The majority of the latter group possessed two tufts $(-33 \%)$, whereas around $12 \%$ even exhibited more than two tufts. In some MTCs, it was hard to differentiate whether two closely adjacent tufted dendrites formed one or two dendritic tufts. In the majority of MTCs, the distinction was possible, however. The ratio between MTCs bearing one dendritic tuft and MTCs possessing two dendritic tufts was almost 1:1 (31 vs. 33 cells). The existence of both MTC types in larval Xenopus OB has already been described by Nezlin and colleagues (Nezlin and Schild, 2005). To the best of my knowledge, this work is the first to systematically classify the different morphologies, dendritic configurations, and ratios of individual MTC subtypes in Xenopus laevis. 
The main question that arises given the morphologies of MTC in the LC is directly related to their primary dendrites and tufts: Do singular tufts indicate uni-glomerular input to MTCs and are multiple tufts a sign of multi-glomerular input?

The calcium imaging experiments of postsynaptic glomerular responses gave an impression about the possible sizes of functional glomeruli. The range of glomerular cross-sectional areas I observed was very broad. Some glomeruli were the size of JGC somata or smaller. Others had cross-sectional areas of $600 \mu \mathrm{m}^{2}$ or more. This variability was also observed among MTC tufts and could be a direct representation of the heterogeneous glomerular sizes. In tadpoles of developmental stage 54, Nezlin and colleagues demonstrated that ORN axons and MTC tufts are the main components contributing to the ovoid glomerular neuropil (Nezlin et al., 2003). Already in early electron-microscopy studies of glomerular ultrastructure in mammals the extensive arborizations of MCs and TCs, which occupied major parts of the glomerular volume, were stressed out. MC and TC dendrites form numerous synapses with ORN axons but also with JGCs that surround glomeruli in a shell-like manner (Kosaka and Kosaka, 2005; Pinching and Powell, 1971a, 1971b). AMCs in the rodent AOS also exhibit extensive dendritic arborizations. Due to the lack of clearly delineated glomerular borders, it is not easy to state whether AMC tufts and $A O B$ glomeruli are comparable in size (Belluscio et al., 1999; Larriva-Sahd, 2008). The findings of Nezlin and colleagues (Nezlin et al., 2003) and my results of the Calcein AM dye injections of MTCs in the LC support the idea that Xenopus MTC tufts cover big portions of the glomerular volume and are to a certain extent predictive for overall glomerular size. I could observe individual glomeruli that were innervated by differently-colored but similarly-sized intermingled dendritic tufts (Figure 6). The glomerular volumes derived from the functional calcium imaging experiments also match well to the MTC tufts' diameters. However, concrete comparisons between tuft and glomerular sizes will only be possible once the glomerular boundaries can clearly be labeled, for instance, by genetically labeling a particular ORN/VRN species (Mombaerts, 1996; Wagner et al., 2006).

\section{Multi-glomerular innervation of MTCs in Xenopus - parallels to the rodent AOS wiring logic}

Assuming that the tuft sizes are representative for the glomerular volume, the MTCs I reconstructed can be divided into different functional subtypes: Group one that receives input from one anatomically confined glomerulus and group two that receive input from multiple glomeruli. In the rodent MOS, uni-glomerular input is usually homogeneous and from one ORN species expressing a single odorant receptor (Mombaerts, 2006). Multi-glomerular innervation by individual MTCs was mainly addressed by studies investigating the wiring logic in the rodent AOS. In those studies, the concept of homotypic or heterotypic wiring of MTCs with their parental glomeruli was discussed (Belluscio et al., 1999; Del Punta et al., 2002; Rodriguez et al., 1999). While the concept of homotypic wiring (MTCs collecting input from 
VRN species expressing the same VR) was proposed early on by Del Punta and colleagues, nowadays there is good evidence, that besides homotypic wiring (Del Punta et al., 2002) a selective heterotypic wiring pattern exists that has been described in the V1R expressing domain of the AOB (Wagner et al., 2006). Individual AMCs received glomerular input from VRN species expressing different V1Rs. However, AMCs seemed to selectively wire with V1Rs of the same phylogenetic subfamily i.e., receptors of high amino acid sequence homology (Wagner et al., 2006). The high ratio of MTCs that projected tufts to multiple distinct glomeruli in my work (42\%), rules in the possibility, that multi-glomerular wiring patterns could play a fundamental role in odor processing of Xenopus. If the multi-dendritic MTCs in Xenopus should also operate as they do in the rodent AOS, this would be another strong support for the hypothesis that the LC of larval Xenopus represents an AOS-like olfactory subsystem. However, it is not clear yet if this is the case and to gather evidence would require genetic labeling of individual VR-type ORN populations as done by Wagner and colleagues. Another option would be an experiment combining presynaptic calcium imaging with electroporation of single (multi-glomerular) MTCs as we published in (Weiss et al., 2018). I performed preliminary trials of this pilot experiment, but no conclusive data was available yet at the time of my thesis submission. The experiment does work though and will hopefully soon unravel whether MTCs receive glomerular input of differently tuned glomeruli.

\section{Developmental pruning as a reason for MTC primary dendrite branching patterns}

There were several additional morphological features I observed among the MTCs in larval Xenopus LC, that resembled features of AMCs in the AOB of rodents (Larriva-Sahd, 2008). The apical primary dendrites of multi-glomerular MTCs mostly originated from a common primary dendritic trunk emerging from the soma, which quickly branched into multiple primary dendrites. Similarly as in AMCs of the rodent AOB (Larriva-Sahd, 2008), the tufted dendrites contributing to the glomerular postsynaptic part could emerge from every imaginable position along the primary dendritic tree. I often observed a bifurcation of the initial primary dendrites and subsequent projection of the branches towards two distinct glomeruli. Most of the time, the initial two dendrites branched even more until terminating in their target glomerulus. Instead of following the initial dendrite's trajectory towards the proximal glomerulus, some of those subbranches turned and innervated the opposite, more distant glomerulus. Different branching patterns of the primary dendritic tree could be categorized with dendrites emerging from every subbranch, innervating either of the glomeruli. These diverse wiring patterns in Xenopus could be seen as a result of glomerular formation, similar to some of the processes described in the olfactory system of rodents. Once the presynaptic scaffold of the glomerular map formed, MCs and TCs in the MOB form extensively branched primary dendritic trees towards the GL early in development (Blanchart et al., 2006; Lin et al., 2000). According to recent findings, the $\mathrm{MC}$ primary dendrites in the rodent $\mathrm{MOB}$ would then form dendritic tufts and synapses with neighboring glomeruli (Nishizumi et al., 2019). In mature AMCs of the rodent AOS (which might be more comparable to Xenopus multi-glomerular MTCs), 
innervated glomeruli can be more than $100 \mu \mathrm{m}$ apart from each other (Larriva-Sahd, 2008; Takami and Graziadei, 1991). Consequently, mature AMCs do certainly not innervate the most proximal glomeruli, as MCs in the MOS (Nishizumi et al., 2019). It is imaginable, that AMCs branch very extensively during development and form connections with a lot of glomeruli among the glomerular array. After this initial phase, excessive branches could be eliminated via pruning mechanisms, as observed in the MOB of rodents (Lin et al., 2000). Similar pruning mechanisms could explain the variability in primary dendritic branching patterns of AMCs and MTCs in the LC of Xenopus laevis. Independent of the primary dendritic branches' trajectory during development, once they wire with their 'target' glomeruli tufts will be stabilized and not eliminated by pruning. While the pruning in homotypically-wired MTCs is more easily imaginable, it is fascinating to contemplate about the underlying mechanisms that would instruct a selectively heterotypic MTC to keep connected with glomeruli of different receptor identities. Since glomeruli formed by individual VRN species can be redundant (Wagner et al., 2006), these MTCs would potentially need to selectively eliminate dendritic connections between a number of glomeruli with different receptor identities. Spontaneous activity patterns of spiking, synaptic release, and second messenger fluctuations are correlated with the spontaneous activity of the particular odorant receptor expressed. Those odorant receptor-dependent, spontaneous activity patterns are elementary for glomerular map formation and maturation (Nakashima et al., 2013, 2019). In the AOB, the glomeruli of different receptors of the same receptor subfamily might display different tuning properties (Hammen et al., 2014; Wagner et al., 2006). The spontaneous activity patterns of two distinct receptors could thus vary. If glomerular pruning in the AOB followed similar mechanisms as Nakashima and colleagues proposed (Nakashima et al., 2013, 2019), the selective heterotypic wiring (Wagner et al., 2006) would be challenging to explain since correlated spontaneous activity seems to be the common denominator of proper glomerular wiring (Nakashima et al., 2013, 2019). However, it could be that overarching common patterns in spontaneous activity are shared between phylogenetically similar VR families. These patterns might make them recognizable as suitable targets by selective heterotypic MTCs (Wagner et al., 2006).

\section{MTC secondary dendrites as targets of GC inhibitory input}

Apart from the primary glomerular dendrites, the multi-glomerular MTCs in larval Xenopus possessed additional dendrites that did not bear tufts, which in the rodent AMCs are also called accessory dendrites (Larriva-Sahd, 2008). Several of them emerged from individual primary dendritic branches or from the common primary dendritic trunk. A striking feature of the bi-glomerular subtype B II (Figure 5) in Xenopus was the existence of long accessory dendrites projecting medially into the plexiform layer. The dendrites resemble the caudal and rostral accessory dendrites observed in AMCs of the rodent AOB (Larriva-Sahd, 2008). 
In contrast to AMCs of the rodent $A O B$ where those dendrites often emerge from the soma (Larriva-Sahd, 2008), I observed long accessory dendrites mainly branching off the primary dendritic tree. In addition to the long variants, shorter accessory dendrites were found both emerging from the primary dendritic branches, but also from the basal side of the soma. Secondary dendrites of MTCs in the MOB of rodents are known to form synaptic contacts with local interneurons, especially with the predominant inhibitory type of the OB, the GCs (Price and Powell, 1970; Shepherd et al., 2007). GCs of the AOB similarly as their counterparts in the MOB form dendro-dendritic reciprocal inhibitory synapses with AMCs (Hayashi et al., 1993; Jia et al., 1999; Taniguchi and Kaba, 2001). Two types have been reported, external GCs which synapse with the soma or glomerular dendrites of the AMCs (Larriva-Sahd, 2008; Mori, 1987; Moriya-Ito et al., 2013) whereas internal GCs synapse on more distal dendritic compartments (Larriva-Sahd, 2008). In larval Xenopus laevis, GCs are not as well investigated as in rodents or fish, but they were shown to be similar in morphology, distribution, and functional properties (Lin et al., 2007; Nezlin and Schild, 2000; Zhang et al., 2016). No matter whether Xenopus MTCs are more similar to rodent MCs, TCs, or AMCs, the observed dendritic specializations likely offer sites of inhibitory input by GCs. Further ultrastructural and functional experiments in our model system will be necessary to elucidate whether the dendro-dendritic synapses formed between accessory dendrites and GCs rather operate self-inhibitory as in the rodent AOB (Mori, 1987) or have a dynamic repertoire of inhibitory actions ranging from sculpting individual MTC output, to coordinating entire neuronal ensembles across the OB as in the rodent MOB (Egger and Urban, 2006; Schoppa and Urban, 2003). 


\section{OR (cAMP independent) \\ V2R (multiglomerular) \\ V2R (uniglomerular) \\ $\mathrm{V} 1 \mathrm{R}$ (uniglomerular) \\ V1R (multiglomerular)}

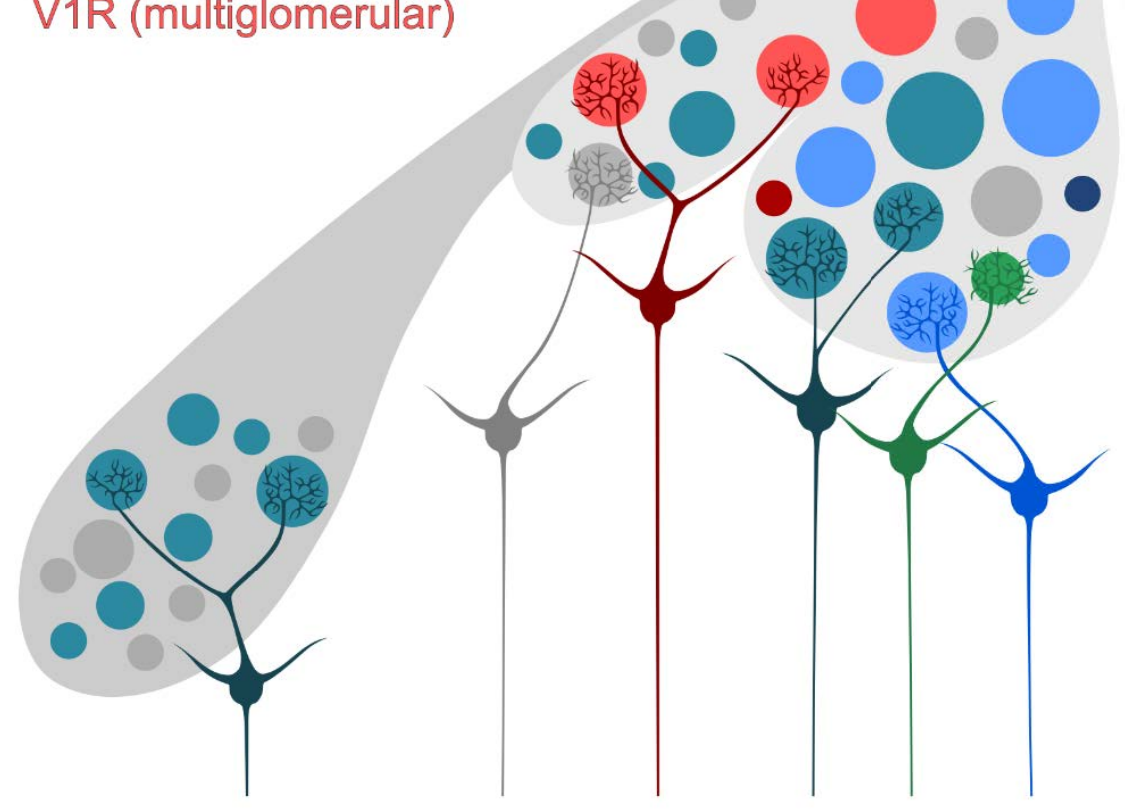

Schematic 19 MTC glomerular wiring strategies and possible odorant receptors involved

\subsection{UNI- AND MULTI-GLOMERULAR MTC WIRING PATTERNS AS A RESULT OF DIFFERENT V2R RECEPTORS}

While I have found several good indications pointing to a rodent AOS-like organization of the lateral olfactory stream in larval Xenopus laevis, the similar ratio of MTCs innervating one or two glomeruli does not seem to fit to the hypothesis at first sight. Several of the Xenopus MTCs I observed, resemble MCs or TCs of the rodent MOB, innervating one glomerulus with their primary dendritic tuft (Nagayama et al., 2014; Pinching and Powell, 1971a). In rodents, this type of neuron has so far almost exclusively been described in the MOS in combination with CAMP-dependent signal transduction and OR-type receptor expression of their parental glomeruli (Munger et al., 2009; Nagayama et al., 2014). The lateral olfactory stream in Xenopus is devoid of glomeruli/ORN axons that use cAMP-dependent signal transduction (Gliem et al., 2013). It cannot be ruled out completely, that OR-type glomeruli with cAMPindependent signal transduction would be present in the LC. In fact, there are reports of an OR-expressing, non-ciliated ORN population, which projects to the AOB in rodents and uses TRPC2 mediated signal transduction (Lévai et al., 2006). It is imaginable that also in those ORNs, the OR would dictate uni-glomerular axonal wiring. Too few is known about this ORN species to draw any conclusions. Rodents are among the most commonly used animal models in olfactory research. However, their MOS and consequently, the MTC types are 
mostly representative of air-borne odor detection systems (Bear et al., 2016; Munger et al., 2009). In zebrafish, most MTCs are innervating one glomerulus with their multiple primary dendrites (Fuller et al., 2006; Wanner et al., 2016). Consequently, zebrafish possess a cAMPindependent, amino acid-sensitive subsystem with uni-glomerular MTCs (Friedrich and Korsching, 1997; Fuller et al., 2006; Sato et al., 2005; Wanner et al., 2016). What could be the reason that some V2R-based olfactory subsystems exhibit mainly multi-glomerular MTCs (rodent $\mathrm{AOB}$ ) whereas others are dominated by the uni-glomerular MTC type? A possible explanation might be that though they are structurally related, OlfC receptors and mammalian V2Rs are phylogenetically distant receptors (Alioto and Ngai, 2005, 2006). An intriguing hypothesis could be that the $\mathrm{V} 2 \mathrm{R}$ receptor family's sequence diversity (including OlfC) results in different axonal wiring strategies depending on the individual receptor. ORNs that express OlfC receptors in zebrafish (and maybe other teleosts as well) could be an example of this clade's wiring logic in which axons coalesce to one glomerulus, and convey their input to a set of uni-glomerular MTCs. Later diverging 'mammalian'-like V2Rs in rodents form multiple glomeruli enabling cross-channel integration from several glomeruli by multi-glomerular MTCs (Belluscio et al., 1999; Rodriguez et al., 1999; Takami and Graziadei, 1991; Wagner et al., 2006). It is tempting to hypothesize that the diversity in V2R repertoire in Xenopus containing both more ancestral fish-like and later diverging V2R variants (Hagino-Yamagishi et al., 2004; Syed et al., 2013) would result in co-existence of both wiring logics. In the principal cavity of larval Xenopus, more ancestral V2R variants are expressed (Syed et al., 2013). That would mean, this ancestral receptor subgroup could possibly be divided further by their uni- or multi-glomerular wiring strategy. It is imaginable that the previously discussed selective co-expression of V2Rs with a V2R group $\mathrm{C}$ member might be predictive for one of the wiring patterns. In larval Xenopus, a subset of V2Rs that are more similar to OlfC receptors could contribute to the uni-glomerular wiring logic, whereas other ancestral 'fishlike' but later diverging V2Rs might be the source of the multi-glomerular wiring logic. The number of glomeruli that were maximally innervated by MTCs in Xenopus in this study was four, and the innervation of two glomeruli per MTC was the most common variant (33\%). This intermediate number of innervated glomeruli in amphibians compared to mammalian AMCs of the V2R domain (Larriva-Sahd, 2008; Takami and Graziadei, 1991) could lead to the impression, that there was a trend to increasing number of glomeruli, the later the V2R family diverged in vertebrates (Shi and Zhang, 2007). However, this linear relationship solely based on phylogeny seems very unlikely, since actually in the majority of other teleost fish investigated, MTCs form synapses with several glomeruli, often more than two (Dryer and Graziadei, 1994; Oka, 1983). Whether V2R-based axonal wiring is the basis of these MTC morphologies is not known. One way to interpret my results of the MTC morphological analysis is that at least two different wiring logics co-exist in the lateral olfactory system of larval Xenopus. One of them is more rodent AOS-like with MTC cross-channel integration from multiple glomeruli (Wagner et al., 2006). The other is based on singular glomerular 
innervation from pre- and postsynapse in a more labeled line like configuration (Mombaerts, 2006; Mombaerts et al., 1996). Those different wiring patterns could be instructed by different groups of V2Rs that do not necessarily need to match the phylogenetic subgroups.

One could argue, that V1Rs might as well be suitable candidates for some of the observed wiring phenomena in Xenopus since they are supposedly using CAMP-independent signal transduction in rodents (Munger et al., 2009) and are found in the larval Xenopus MOS (Syed et al., 2013). However, the variability of the glomerular map, as well as the high prevalence of multi-glomerular MTCs in the amino acid-sensitive mediolateral lobe of the LC, strongly suggests that at least a part of the Xenopus multi-glomerular MTC population might be receiving input from V2Rs. It cannot be excluded that V1R-based glomerular wiring patterns independently contribute to either the uni- or multi-glomerular MTC population. If Amphibian V1Rs were similar to mammalian V1Rs in that sense, the multi-glomerular phenotype of MTCs could occur preferentially (Wagner et al., 2006).

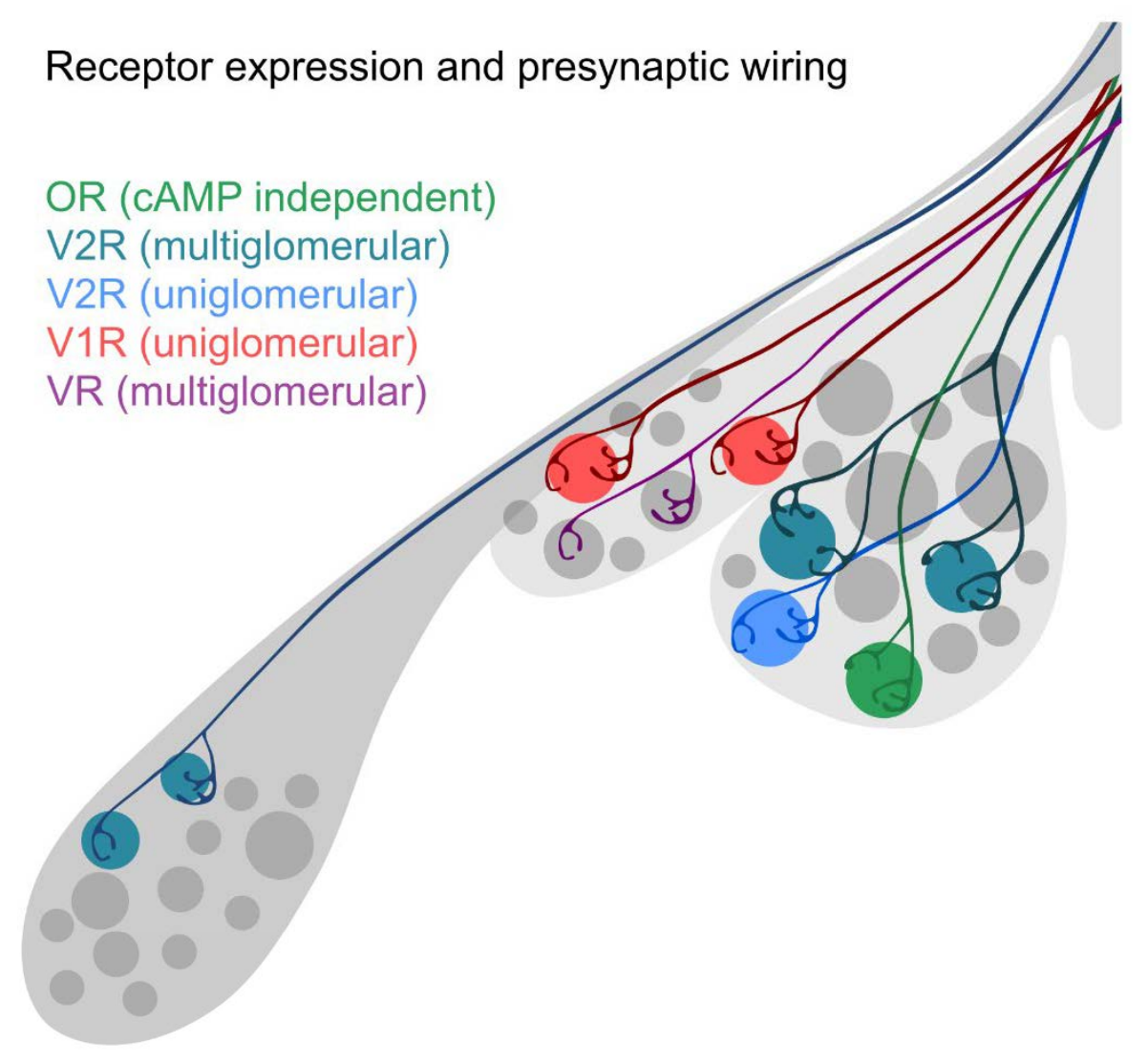




\subsection{A 'THREADED LABELED LINE' (THRLL) MODEL OF GLOMERULAR INFORMATION PROCESSING}

The axonal bifurcation and multi-glomerular innervation by single ORNs in larval and adult Xenopus laevis have been a challenging additional level in the task of dissecting the wiring strategy of Xenopus OB (Weiss et al., 2019) Especially, since a big part of the relevant literature for axonal wiring in either the MOS or AOS of rodents or fish have not reported multi-glomerular innervation by individual ORN axons (Belluscio et al., 1999; Dynes and Ngai, 1998; Klenoff and Greer, 1998; Mombaerts et al., 1996; Rodriguez et al., 1999). I only found one study by Larriva-Sahd using Golgi stainings in the AOS of rodents that revealed that bifurcations, as well as multi-glomerular innervation patterns by individual axons, do exist in almost $20 \%$ of all VRNs. This is a considerable ratio and seems to be a basic form of axonal wiring in the AOS in addition to the other types observed. Unfortunately, Larriva-Sahd does not specify in his work which domain of the AOB the branching axons were observed in (Larriva-Sahd, 2008). Very recent results of our lab have clearly shown that multi-glomerular innervation by individual ORN axons is a more common feature than previously anticipated. Among amphibians, it seems to be very prevalent (Weiss et al., 2019). In both larval and adult Xenopus laevis, the two most frequent mature patterns are innervation of one or two glomeruli (Hassenklöver and Manzini, 2013). Innervation of three or more glomeruli occurred rarer (Hassenklöver and Manzini, 2013; Weiss et al., 2019). The ratios of these particular wiring patterns match well with the MTC glomerular innervation patterns I observed on the postsynaptic side. Could it be, that the odor information of anatomically distinct glomeruli formed by bifurcating axons is simply converged again onto multi-dendritic MTCs? Or are glomeruli duplicated to provide more diverse possibilities of selective heterotypic or homotypic wiring by MTCs, as seen in the rodent AOS (Del Punta et al., 2002; Wagner et al., 2006). This question could not be solved conclusively by the experiments I performed during my thesis and will most likely be one of the final puzzle pieces to understand the complex circuitry of the lateral olfactory stream. Whichever wiring strategy it might turn out to be and whichever odorant receptor is involved, the observation of this phenomenon in $10-20 \%$ of rodent VRNs gives the impression, that also this wiring strategy could be an AOS-like phenomenon (Larriva-Sahd, 2008). Xenopus laevis like maybe many other Amphibians represent animals, in which MOS and AOS like subsystems are partially (Gliem et al., 2013) but not yet fully segregated as in rodents (Munger et al., 2009). The co-existence of both AOS- and MOS-like wiring patterns in the Xenopus MOB might thus account for the observed heterogeneity in neuronal connectivity on both the pre- and postsynaptic side. The bifurcation and multi-glomerular innervation by individual ORN species might be a yet overlooked, but fundamental wiring principle of certain VR subgroups across vertebrate species (Hassenklöver and Manzini, 2013; Larriva-Sahd, 2008; Nezlin and Schild, 2005; Weiss et al., 2019). If individual ORNs' axons innervated several anatomically distinct glomeruli, but those were postsynaptically all composed of the tufts contributed by a set of multi-glomerular sister 
MTCs, the wiring would not be that different from a classical labeled line configuration as in the rodent MOS. The 'threading' of this labeled line might provide redundant glomeruli from one ORN species that can be integrated differentially by different sets of MTCs. It is imaginable that in addition to this 'threaded labeled line,' wiring logic, heterotypic MTCs exist that integrate input from several of those different threads. This cross-channel integration would resemble the connectivity of AMCs in the rodent AOS (Wagner et al., 2006), and might co-exist alongside a 'threaded labeled line' or the uni-glomerular wiring logic.
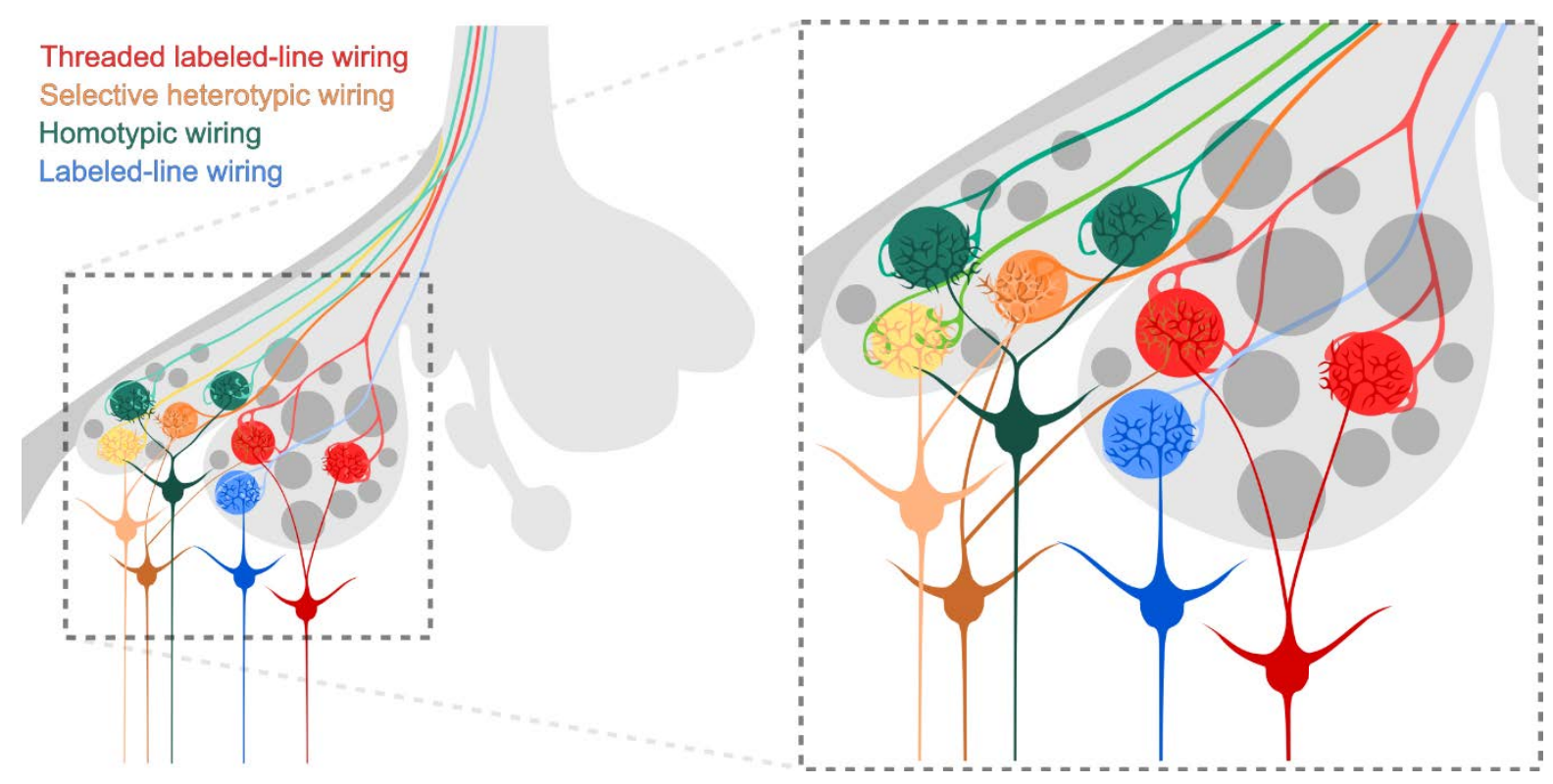

Schematic 21 Possible co-existence of different wiring strategies in the LC of larval Xenopus

\subsection{NBT EXPRESSION AS A MARKER OF A SUBSET OF MTCS IN LARVAL XENOPUS \\ $\mathrm{NbT}$ expression as a marker of MTC maturity and axogenesis.}

One of the crucial steps to unravel the larval OB's organization and wiring principles is to characterize the MTC population further. The target regions of individual subsets of projection neurons can be indicative for their function and behavioral relevance, as seen in the MCs and TCs of the MOS in rodents (Igarashi et al., 2012; Nagayama, 2010). The NbT-Katushka transgenic reporter line I used, seems to be well suited to tackle these questions in larval Xenopus. My results revealed that NbT-positive neurons in the larval bulb display MTC morphologies. However, the sparse cell electroporations I performed could not fully answer, whether NbT-positive neurons are mainly of the multi- or uni-glomerular MTC type, or both. The $3.8 \mathrm{~kb} \mathrm{NbT}$ promotor fragment used to generate the transgenic reporter line drives Xenopus neuronal beta-tubulin type II expression. Neuronal beta-tubulin II was shown to be associated with neuronal identity and axonal development in frog embryos (Moody et al., 1996). This is in contrast to mammalian systems, where beta-tubulin class III is a selective marker for neurons (Moody et al., 1996). Xenopus NbT expression peak in neurons across the brain that undergo phases of axonal growth during early development (Moody et al., 1996). I observed multi-glomerular wiring patterns in a couple of NbT-positive MTCs. The 
correlation between axonal development and $\mathrm{NbT}$ expression could at first sight hint to the proposed hypothesis that there might be a considerable number of immature MTCs and (proto-)glomeruli present in the larval OB. The activity of the NbT promotor, which was visible by fluorescent protein expression, might be a marker of neuronal maturation status. This would particularly apply for the MTC population since most other cells in the MCL should not possess extensive axons (Igarashi et al., 2012; Nagayama et al., 2014). I performed calcium imaging experiments in the whole olfactory system explant of larval NbT-Katushka tadpoles using amino acids as stimuli. I wanted to investigate whether NbT-positive MTCs are actually wired with their glomeruli. It was impossible to evaluate, whether irresponsive NbT-positive MTCs were just not tuned to the amino acid stimuli used or not receiving synaptic input at all. However, I could show that a set of NbT-positive MTCs does respond to amino acids and displays similar tuning properties as the surrounding NbT-negative neurons of the MCL. Like in the recordings of postsynaptic glomerular responses, the tuning properties of NbT-positive MTCs exhibit dominant responses to certain amino acids' structural features. I could not observe a significant number of MTCs that were broadly responsive to structurally different amino acids. Given the multi-glomerular innervation pattern of Xenopus MTCs this could have been theoretically possible as a form of glomerular cross-channel integration as in the rodent AOB (Takami and Graziadei, 1991; Wagner et al., 2006). My findings confirm that at least a set of NbT-positive MTCs is synaptically connected to their glomeruli and that those MTCs are also selectively tuned to individual amino acids' structural features.

While there is a lot of research on olfactory map formation on the ORN level (Imai and Sakano, 2007; Mori and Sakano, 2011), few is known about MTC development and which mechanisms underly the proper wiring with the higher olfactory brain centers. Developing MTCs were shown to send their axons towards higher brain centers already before the arrival of the ORN axons in the rodent MOB (López-Mascaraque and de Castro, 2002; LópezMascaraque et al., 1996). Igarashi and colleagues succeeded in reconstructing the extensive axonal projection field of individual MCs and TCs to their respective higher brain centers in adult mice (Igarashi et al., 2012). Interestingly, transsynaptic tracing studies imply that the coarse topological organization of the MTCs' axonal projections is conserved in some higher brain centers whereas, in others, it seems to be spatially reordered (Ghosh et al., 2011). Thus, the question arises, whether glomerular maturation is required before proper axonal wiring of MTCs with their higher brain centers. Could it be that wiring with higher brain centers is predetermined independently of sensory input and the higher brain centers adapt once sensory input arrives?

Our laboratory and others could show that tadpoles of pro-metamorphic stages can behaviorally respond to amino acid stimuli (Hawkins et al., unpublished results) Upon ON transection these responses are lost (Cervino et al., 2017; Terni et al., 2017). After ON axotomy MTC dendritic tufts' complexity decreases, but MTCs persist in the larval OB without 
sensory input for several weeks (Hawkins et al., 2017). Their synaptic connections to ORNs that re-innervate the $\mathrm{OB}$ upon injury restore their tuft complexity and odor-responsiveness to a significant extent seven weeks after transection (Hawkins et al., 2017). This ability of MTCs to persist without sensory input for several weeks is remarkable and might require specialized mechanisms of axonal maintenance or regrowth. One could speculate that Xenopus NbT promotor activity is not necessarily exclusive to immature cells undergoing axogenesis but could be a feature of mature MTCs. Maybe neuronal beta-tubulin II might even grant them their outstanding wiring stability even in the absence of sensory input. Immunohistochemical studies against the expressed fluorescent protein Katushka in combination with single-cell electroporations might help to solve the question of MTC subtypes and whether they all wire with higher brain centers in. Behavioral experiments with larval Xenopus laevis confirm, that the animals can react to amino acids as olfactory stimuli, so at least a set of MTCs must be wired (Cervino et al., 2017; Terni et al., 2017).

\section{$\mathrm{NbT}$ expression in developing MTCs}

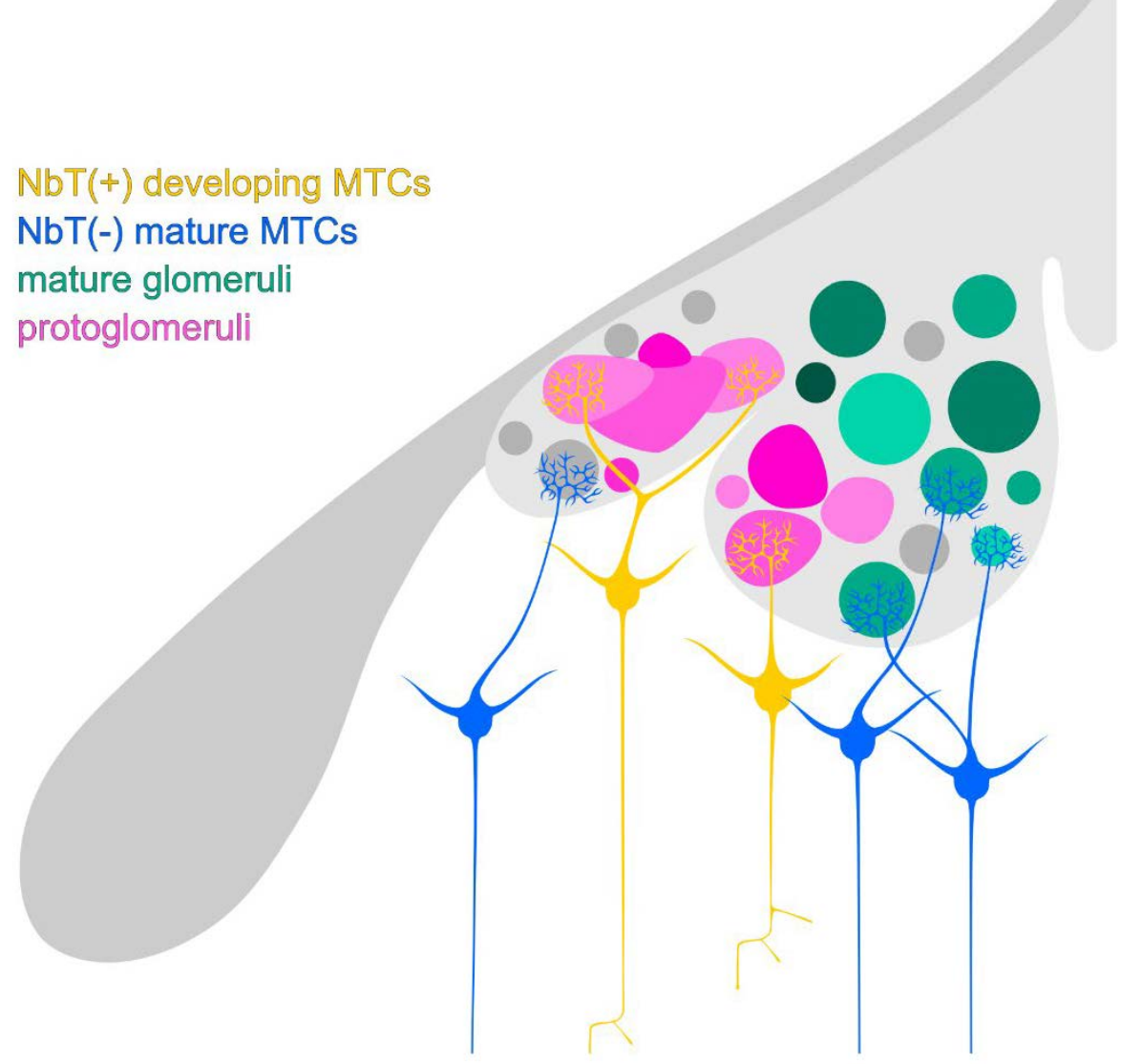

Schematic 22 NbT expression as a possible marker for immature MTCs in a developing olfactory subsystem

MTCs were often not fully traceable in this work due to caveats of the sparse-cell electroporation method. If further experiments with different techniques should reveal that uni-glomerular MTCs only possess short axons with collaterals that do not extend far beyond the $O B$, it might be, that those 'MTCs' in fact are a similar cell type as the ATCs of the AOS in rodents (Larriva-Sahd, 2008). ATCs only receive input from one glomerulus via a single primary dendrite from which accessory dendrites can emerge. The axon collaterals can be 
extensive, but do not seem to project to higher olfactory brain centers (Larriva-Sahd, 2008). I did see several MTCs that could fit those criteria in the larval bulb. In this scenario, the primary output neurons of the Xenopus larval OB could be the multi-glomerular MTCs, whereas the ATC-like cells could act as local interneurons (Larriva-Sahd, 2008). If the uniglomerular MTCs of larval Xenopus MOB should indeed be more similar to ATCs than to projection neurons, it would be interesting to investigate whether there are differences in $\mathrm{NbT}$ expression between those Xenopus ATC suspects and the multi-glomerular, rodent AMClike, MTCs. While both possess axons, the rodent ATCs' projections and collaterals are believed to not extend beyond the OB (Larriva-Sahd, 2008). That could imply that already during development, axogenesis in ATCs might be finished earlier than in projection neurons. Wiring of the mostly local targets in the OB might be followed by a cease in NbT promotor activity in mature ATCs. This should be visible as a decrease or loss of Katushka fluorescence in the transgenic reporter line.

\section{$\mathrm{NbT}$ expression in MCs vs. TCs}

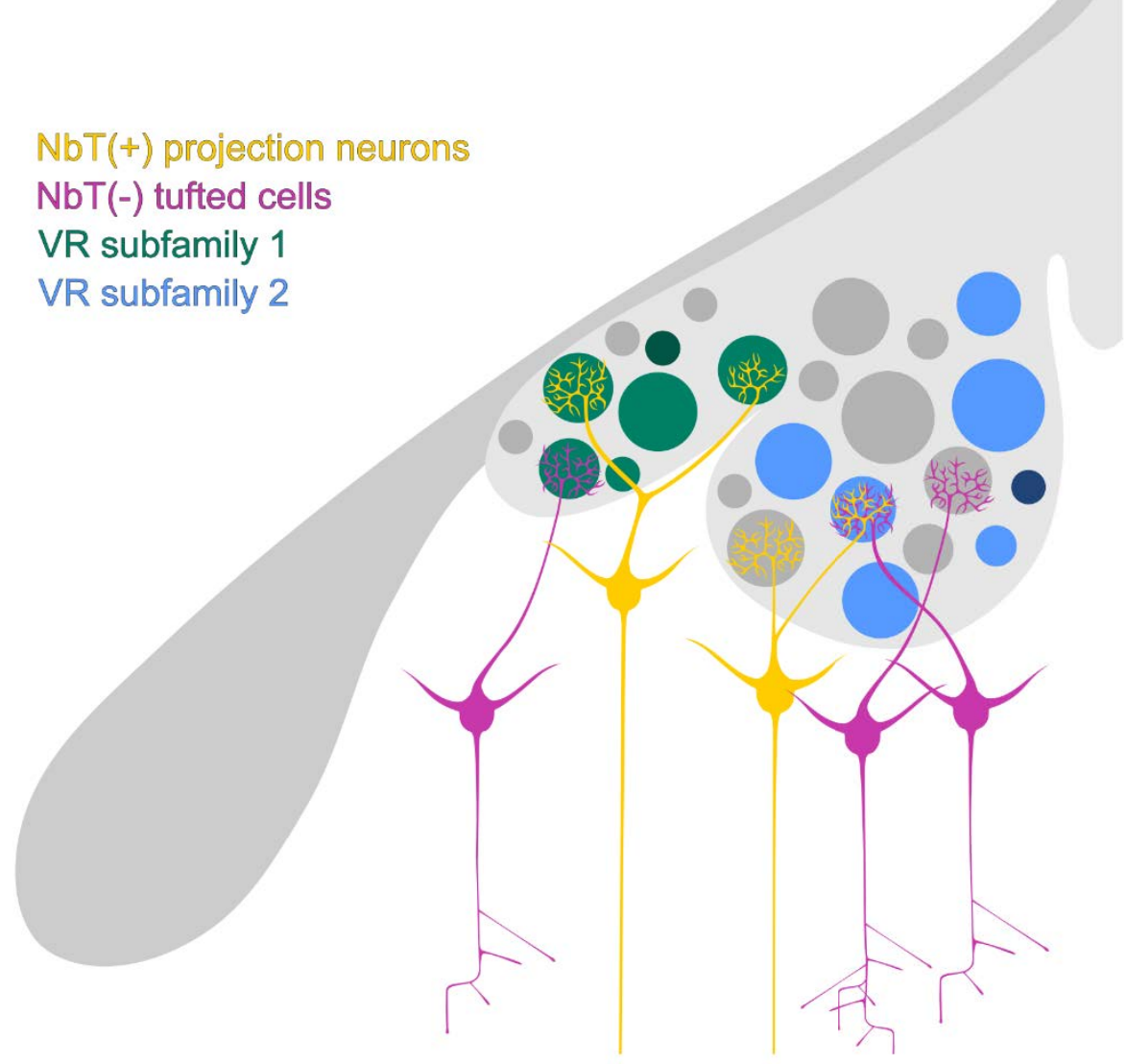

Schematic $23 \mathrm{NbT}$ expression as possible marker distinguishing mature MTCs from a rodent AOS-like TC type

\section{NbT-negative neurons between GL and GCL - MTCs, JGCs and more}

The NbT-negative population of neurons I recorded in the calcium imaging experiments was certainly more heterogeneous than the NbT-positive population. While I injected the Fluo-4 AM calcium indicator into the MCL, it could not be ruled out that GCs and juxtaglomerular 
cells also take up the dye and contribute to the amino acid-responsive population of NbTnegative cells. I was able to measure the tuning profiles of JGCs in the odor mapping experiments of the GL described earlier. Due to the restricted volume of the medioventral LC that I could scan at a reasonable speed, most cells I recorded were in close proximity to the glomeruli, which should justify classifying them as juxtaglomerular cells. The population of JGCs in the MOB of rodents is very diverse and consists of several different cell types varying in their morphology, function, neurotransmitter and other molecular markers (Kosaka and Kosaka, 2005, 2016; Kosaka et al., 1998; Nagayama et al., 2014). In the ventral bulb of larval Xenopus, the number of PGs, the GABAergic majority of juxtaglomerular cells, cover around 175+-20 cells (Nezlin and Schild, 2000). Other markers that have been validated in JGCs in Xenopus laevis are Tyrosine hydroxylase, an enzyme involved in dopamine synthesis (González et al., 1993) and the calcium-binding proteins Calretinin and Calbindin (Morona and González, 2013). Nezlin and colleagues could observe JGCs they classified as PGCs in larval Xenopus that covered entire glomerular clusters with their dendritic projections but possessed no tuft like arborizations that contributed to the ovoid shape of the glomerular neuropil (Nezlin et al., 2003).

\subsection{ODOR INFORMATION PROCESSING IN THE OB NEURONAL NETWORK}

The glomerular module in larval Xenopus - no apparent 'sharpening' of odorant tuning from the GL to the MTC level

In mammals, PGC dendrites usually cover the entire volume of the one sometimes up to two glomeruli they innervate in the MOB (Kosaka et al., 1998; Nagayama et al., 2014; Pinching and Powell, 1971b). Their particularly extensive arborizations contribute considerably to the postsynaptic glomerular neuropil (Kosaka et al., 1998; Pinching and Powell, 1971b). My findings that JGCs in larval Xenopus are similarly tuned to amino acid odorants as the postsynaptic MTC tuft part of glomeruli goes in accordance with other functional studies investigating individual glomeruli and their associated juxtaglomerular cells in rodents (Homma et al., 2013, 2019; Kikuta et al., 2013). Since a part of the PG population receives direct synaptic input from ORNs and are in general extensively coupled with MC and TC dendrites (Wachowiak and Shipley, 2006) their apparent odorant responses are similar to their parental glomeruli (Kikuta et al., 2013). This similarity in odor tuning holds as well true for the $A O B$, in which the sparse PGC population is believed to be mostly involved in intraglomerular inhibition via dendro-dendritic synapses (Mori, 1987). The similarity in odorant tuning between MTC tufts and JGCs I observed in Xenopus would theoretically fit the odor tuning properties of the MOB glomerular modules as proposed by Kikuta and colleagues (Kikuta et al., 2013). However, PGCs in larval Xenopus can exhibit dendritic projection fields spanning entire glomerular clusters (Nezlin et al., 2003). The similarity in odor tuning of PGCs and glomeruli I observed in larval Xenopus as well as PGCs' connectivity to so many glomeruli (Nezlin et al., 2003) seems in harsh contrast to the mostly uni- 
glomerular association of PGCs in the rodent MOB at first sight (Kosaka et al., 1998; Nagayama et al., 2014; Pinching and Powell, 1971b). Taking into account the possible redundancy of glomeruli in larval Xenopus due to the 'threaded labeled line' wiring strategy or rodent AOS-like wiring, the extensive dendritic projections of JGCs across the GL could be a result of their connectivity with multiple parental glomeruli. PGCs in larval Xenopus might thus operate between large sets of glomeruli via autoinhibition as in the rodent AOB (Mori, 1987; Mugnaini et al., 1984; Quaglino et al., 1999). The extensive dendrites of JGCs could also exert lateral inhibition. This might explain the observed similarity in odor tuning of JGCs and entire sets of postsynaptic glomeruli. Why JGCs somatic odor tuning seemed to be more selective to functional subgroups of amino acids than the actual glomerular neuropil remains unanswered.

I could not observe a clear 'sharpening of odor tuning' on the MTC level in Xenopus, as observed in the rodent MOB (Kikuta et al., 2013). Depending on the response threshold, odor tuning was somewhat similar or broader on the MTC level. In the MOB, glomerular input is processed via parallel channels of MCs and TCs that convey distinct qualities of the spatiotemporal sensory information (Igarashi et al., 2012; Nagayama et al., 2010). The rodent AOS-like organization of the lateral olfactory stream might be the underlying reason for this conserved or broader odor tuning of MTCs in larval Xenopus.

\section{The glomerular module in larval Xenopus - Conservation of parental glomerular odor tuning or integration from different glomerular channels on the MTC level}

Feature extraction and processing of the raw sensory input are often achieved by lateral inhibition between glomerular modules mostly on the GL level, leading to a preprocessed odor representation in rodents (Aungst et al., 2003; Cavarretta et al., 2016; Economo et al., 2016). The cross-channel integration by multi-glomerular AMCs of the rodent AOS is in sharp contrast to this odor processing strategy (Takami and Graziadei, 1991; Wagner et al., 2006). Instead of extracting information about molecular features, concentration, and spatiotemporal dynamics, the AOS wiring logic seems to serve the detection and integration of behaviorally relevant chemicals or molecular blends (Hammen et al., 2014). MTCs that integrate the input of glomeruli from different ORN species could serve to only convey odor information, when several glomeruli are simultaneously activated. Wagner and colleagues argued, that this type of molecule detection would avoid behaviorally irrelevant noise by activation of individual glomerular channels only (Wagner et al., 2006). The conserved or even broader odor tuning of MTCs in Xenopus could thus be a result of similar integrative operations via multi-dendritic MTCs. While MTCs showed a clear preference to certain individual amino acids' structural features, when lowering the response detection threshold, the odor tunings of the MTC were more diverse. The dominant tuning profile of MTCs could mirror the MRR i.e., molecular binding pocket properties of the VR subfamily from which the individual MTC integrates its information. The diversity on the level of smaller odorant response amplitudes might be due 
to effects caused by the activation of smaller subsets of the glomeruli that are integrated by each MTC. The (dominant) odor tuning of individual MTCs might carry the information about the receptor family subgroup in the form of the dominant, high amplitude responses. 'Smaller responses' might later on either be disregarded or contain additional relevant information. This would represent an interesting form of multiplexing on the MTC population level.

Especially, on the level of postsynaptic glomeruli and JGCs, a strong selectivity of neurons towards amino acids with either basic/aromatic or long-chain-neutral sidechains could be observed. This might be a correlate of different underlying odorant receptor subfamilies. On the level of MTCs, this segregation was conserved, and NbT-positive MTCs also responded preferentially to single or few amino acids of one of the amino acid mixes. Taking the existence and high prevalence of multi-glomerular MTCs into account, this could imply that MTCs integrate information from glomeruli of similar tuning properties, but do not integrate information of glomeruli with very dissimilar tuning. This conservation of odor tuning on the MTC level could support the existence of homotypic glomerular innervation by larval Xenopus MTCs similarly, as observed in the rodent AOB (Del Punta et al., 2002). The selective tuning of glomeruli, PGCs, and MTCs to molecular features of certain amino acid subgroups, could point to common input from ORNs that express specific odorant receptors or sub-families. Especially the proposed 'threaded labeled line' glomerular wiring logic could explain conserved odor tuning on the different levels of odor information processing in the larval Xenopus OB.

\section{Temporal coding of odor information in an AOS-like olfactory system}

Odor tuning and population-based coding strategies are just one of the ways the larval OB of Xenopus might encode or process odor information. Several studies, not restricted to rodents, have proposed, that the OB can encode odor qualities like odor identity and concentration by a latency code carried mainly by the output neurons. To be more precise, first spike latencies have proven to serve as a robust read-out of odor identity (Cang and Isaacson, 2003; Junek et al., 2010; Margrie and Schaefer, 2003; Schaefer and Margrie, 2012; Spors and Grinvald, 2002; Spors et al., 2006) or even odor concentration (Geramita and Urban, 2017; Junek et al., 2010). In contrast to absolute latencies, which always need an external reference frame to be interpreted by the brain, sequences of neuronal activation can be interpreted in a manner that is independent of the individual neuron's absolute latency. This latency rank coding was indeed discovered in larval Xenopus laevis MTCs and represents an innovative first spike latency-based coding method, that does not necessarily need an external reference frame (Junek et al., 2010) as in the rodent MOS (Spors and Grinvald, 2002; Spors et al., 2006). Especially in the rodent olfactory system, external reference frames for latency coding have been shown to play a significant role (Cang and Isaacson, 2003; Laurent et al., 2001; Margrie and Schaefer, 2003). Periodic, oscillatory patterns intrinsically generated by the olfactory network or superimposed onto it, serve as powerful external reference frames for 
latency coding across species (Kay, 2015). Whether such oscillatory rhythms exist in Xenopus OB is not known at the moment. Junek and colleagues found this latency-based coding strategy in MTCs of the lateral olfactory stream in tadpoles of the same developmental stages I used. It is very intriguing to contemplate how the odor coding mechanisms they observed fit with the hypothesis that that part of the lateral Xenopus MOB might operate in a rodent AOS-like manner. The heterogeneous lengths and trajectories of primary tufted dendrites I observed in the MTCs are not easy to interpret when it comes to time-sensitive processes in the range of milliseconds as in latency coding (Junek et al., 2010). Backpropagation of electrical signals from the soma towards the apical tuft was shown to be possible in MTCs and AMCs (Chen et al., 2006b; Ma and Lowe, 2004; Yuan and Knöpfel, 2006). Active backpropagation by somatic spiking or sub-threshold signal propagation might have the potential to interfere with incoming signals from the other glomeruli, especially in multi-glomerular MTCs (Castro and Urban, 2009; Ma and Lowe, 2004; Urban and Castro, 2005). These highly dynamic, nonlinear computations could provide a plethora of possible output patterns directly related to the morphological and biophysical features of each AMC. On the other hand, the somatic summation of primary dendritic inputs might be affected in multi-dendritic MTCs. If for instance one of the glomeruli was strongly activated by an odorant, the backpropagation of electrical signal into the other primary dendrites could cancel out possible incoming signals from those other glomerular channels (Castro and Urban, 2009; Ma and Lowe, 2004; Urban and Castro, 2005). This might even be of advantage if it was not necessary for all different glomerular inputs to arrive simultaneously to trigger MTC firing. It could provide a 'winner takes it all' like mechanism in which the summed electrical signal that reaches the soma first and triggers spiking defines its first spike latency regardless of all subsequent dendritic computations. However, recent results of Yoles-Frenkel and colleagues propose that the multi-glomerular dendritic configuration of AMCs disfavors fast temporal coding strategies (Yoles-Frenkel et al., 2018). They claimed that AMC input integration works on a slower scale and is too variable to reliably encode input arrival times (Yoles-Frenkel et al., 2018). How do multi-glomerular MTCs and latency coding of odors in larval Xenopus laevis fit? My results might provide a framework for those two concepts not to clash. The coexistence of several wiring strategies in one olfactory subsystem might allow the co-existence of several coding strategies. The labeled line, or rodent MOB-like glomerular module, might thus be the most promising candidate to carry the latency based, fast temporal coding in larval Xenopus (Junek et al., 2010). Since Junek and colleagues used amino acids as odorants in the latter study, this would also imply that a considerable part of the amino acidsensitive MTC population I measured in my work would wire in a labeled line configuration. The threaded labeled line wiring logic might also be imaginable for such a temporal coding strategy.

Chen and colleagues were able to show, that dispersed subsets of MTCs in the larval OB of Xenopus form functionally coupled modules that are associated with one glomerulus (Chen 
et al., 2009). My results regarding the distance between NbT-positive MTC somata and their similarity in odor tuning are in accordance with their study: There seems to be no obvious tight spatial clustering between sister MTCs in larval Xenopus. Spatial clustering of sister MTCs is controversial in rodent MCs (Buonviso et al., 2002; Egaña et al., 2005; Kikuta et al., 2013). It should be noted though, that recent research has revealed, that the so-called sister MCs innervating the same parental glomeruli in the MOB might show similar odor tuning on the level of calcium imaging, but can carry differential information on the finer temporal scale of action potentials that are not necessarily highly correlated (Dhawale et al., 2010). By performing multi-color sparse cell electroporation of MTCs, I managed to label pairs of sisterMTCs innervating the same two glomeruli (Figure 6). These findings, even though low in number, complement the results by Chen and colleagues and indicate that the existence of coupled sister-MTCs might not be restricted to MTCs innervating one glomerulus only (Chen et al., 2009). My concluding hypothesis on the different roles of MTCs in population and temporal coding is that the lateral olfactory stream could incorporate rodent MOB- as well as AOB-like coding strategies (on the population and temporal level) or might even use those wiring logics synergistically. The hypothetical 'threaded labeled line' wiring logic might be an amphibian intermediate solution, that could allow labeled-line-like odor encoding but at the same time offer the possibility of AOS-like cross-channel integration between the module's 'threads.'

\section{SUMMARY}

Olfactory systems of vertebrates have adapted to the various challenges of their environments on both the level of odorant receptors and neuronal wiring. Amphibians like the African clawed frog Xenopus laevis offer a powerful model to investigate how different neuronal wiring and odor information processing strategies emerged among vertebrates during water-to-land transition. In the olfactory bulb the sensory information from the peripheral olfactory organs is conveyed to mitral/tufted cells and their associated neuronal network in anatomical and functional units called olfactory glomeruli. My work aimed to answer the question, how olfactory sensory information is organized on the glomerular level and relayed to the mitral/tufted cells in the lateral olfactory bulb of larval Xenopus laevis. I could reveal the full extent of the olfactory receptor neuron population that innervates the lateral glomerular cluster and provide evidence for an anatomical and possibly functional finer subdivision of the lateral olfactory subsystem. I characterized sulfated steroids as suitable stimuli for mitral/tufted cells of the accessory and the lateral main olfactory bulb. The glomerular odor representation of the postsynaptic network to single amino acid stimuli appears to be very variable and non-stereotypical between animals. I found several similarities of the larval Xenopus lateral glomerular cluster to the rodent accessory olfactory system. Among those were the lack of chemotopy and the independence of tuning and spatial clustering between glomeruli. I characterized the population of mitral/tufted cells associated 
with the lateral glomerular cluster and found major differences in morphology and glomerular connectivity between them. The equal ratio between uni- and multi-glomerular MTCs imply the co-existence of several wiring logics in the lateral olfactory bulb. Neuronal beta tubulin II expression defines a subset of mitral/tufted cells of unknown maturation status, that was in parts responsive to amino acids. Overall, my work suggests, that the lateral olfactory subsystem incorporates several different wiring logics and resembles a rodent 'accessory olfactory system-like' subsystem that is still undergoing developmental processes. My work and further work from our laboratory might hint to the existence of a yet undescribed 'threaded labeled line' wiring logic in the olfactory system of amphibians. The actual connectivity and purpose of these potential wiring and odor information processing strategies remains to be determined.

\section{OUTLOOK}

There are plenty of ways imaginable, how the neuronal elements of the OB circuitry could be connected to form glomerular modules and encode odor information in larval Xenopus laevis. While my work and the work of our laboratory provided several new hints on how the glomerular modules might be organized, genetic tools and transgenic animal lines will be indispensable to disentangle the wiring logic and olfactory information processing. In his book 'What Mad Pursuit' (Crick, 1988), Francis Crick already contemplated about '..., a technique for injecting a single neuron in such a way that all the neurons connected to it (and only those) are labeled would be useful...' (as referenced in Yoshihara, 2002). Today this dream has partially come true through the introduction of (mono-synaptic) transsynaptic tracing methods (Luo et al., 2008; Nassi et al., 2015). While I have experimented with transsynaptic tracing methods based on WGA and vesicular stomatitis virus during my PhD, there is no way yet to label synaptically connected neurons in the olfactory system of Xenopus. I believe that conquering this method in our animal model will provide the final clues how the amphibian olfactory system and its glomerular modules are wired. Transgenic animal lines in which individual ORN or MTC species are labeled, might additionally help to understand the different neuronal subtypes involved in the glomerular module, especially how individual odorant receptor information channels are interpreted in the OB. As a next step, a newly available transgenic Xenopus laevis line that expresses a genetically encoded calcium indicator in NbT-positive neurons would complement my calcium imaging results in the OB neuronal network. Moreover, it would provide the possibility of simultaneous pre- and postsynaptic calcium imaging to investigate odor map transformation on the glomerular level. A combination of single MTC electroporation and odor activity mapping of ORN axon terminals could give valuable insight, whether individual multi-glomerular MTCs receive homotypic or heterotypic odorant receptor input. It will be necessary to find suitable markers or stimuli specific for individual amphibian odorant receptor families (especially V2Rs). That 
way it might be possible to draw conclusions on how individual odorant receptors dictate axonal wiring and glomerular module composition in amphibians or even vertebrates in general.

\section{ACKNOWLEDGEMENTS}

First and foremost, I want to thank my family, Karin, Robert and Johannes Offner as well as my grandparents Susanna and Andreas Löw. Without you and your continuous, loving support I would not be anywhere close to where I am right now. Thank you from the bottom of my heart for everything! I want to also thank all the friends I made on my journey. To my friends from Speichersdorf who are and will always be my home base: Thank you, Felix and Teresa Prischenk, Jonas and Julia Anjol, Hannes and Philipp Höcker, Steffen Hinke, Marco Fett, Christian Nerlich, Alexander Eberl and Sonja Porsch for your support and your patience even when I sometimes go 'off the radar' for months. I hope we stay as connected as we are, no matter where the winds carry us in the future. Thank you to my pirate crew from Erlangen who are still an important part of my life. Julia Kleemann, Anja Pfaus, Jasmin Knopf, Christine Rummel, Felix Bögler, Philipp Kestel, Sebastian ,Wolle‘ Widholz and Lukas Brecht. I hope we will manage to keep the pirates in us alive and sail together for many more years to come. Thank you to the numerous friends I made in Göttingen, from the IMPRS Neurosciences program and beyond. Julio Santos Viotti, Alexander Dieter, Sebastian Jähne, Carlos DuqueAfonso, Luis Ramos Traslosheros-López, Georg Hafner, Michael Feyerabend, Özge Demet Özçete, Diego Giraldo Sanchez, Nicolás Lemuz Diaz, Daniel Keppeler, Fritz Kobe, Christian and Isabelle Vogl, Angie Windisch, Paola Agüi Gonzalez, Sinem Meleknur Sertel, Shama Sograte Idrissi, Susanne Witt and those I forgot. The years with you there were the best years of my life and I would not want to miss any of the mind-opening and fun experiences I made in Göttingen. Especially, I want to thank Lina María Jaime Tobón for the five years of partnership and support. The time with you was wonderful. It was definitely a love worth loving. A big thank you goes to my lab-siblings Sara Joy Hawkins, Lukas Weiss and our beloved adoptive brother Lukas 'Quappo' Wichmann. Without you, the moving process to Giessen and this work would not have been possible. By annoying and supporting each other we have made it through difficult times. I am very grateful that we turned from colleagues to so much more than that. Can't wait for the future adventures of 'the three Idioteers and Quapp'argnon'. I also want to thank my Bachelor/Master students Anna Berk, Daniela Daume and Michelle Endres. Supervising you gave me a perspective and enriched my life here in Gießen. Thank you also to the other Bachelor and Master students in Gießen especially, Melina Kahl, Yvonne Gärtner and Michel Hauschild and also our colleague Anja (Sgt.) Schnecko

I want to thank Ivan Manzini for his support of this work. I am grateful for the trust you had into my abilities and the freedom I had all those years to work very independently. Your downto-earth personality and approachability contributed to the pleasant work ambience that kept 
me going those years. I am also grateful for the continuous financial support you provided from the moment I entered the lab and that I learned many things about lab management, that I would likely not have learned at this early stage elsewhere. I want to thank Thomas Hassenklöver for his scientific supervision throughout my PhD. I am grateful for everything you have taught me and your professionality.

Thank you to my thesis advisory committee members Thomas Dresbach and Silvio Rizzoli. I am grateful for our exciting collaborative projects but also for your guidance and advice until today. I could not have chosen a better committee for myself. A big thank you also to Felipe Opazo, Buket Basmanav from the Rizzoli lab as well as Irmgard Weiss, Julio Santos Viotti and the rest of the Dresbach laboratory.

I want to thank Markus Dietrich and the CNMPB for their great support of my work. The CNMPB has been absolutely crucial to my work. Thank you also to Regina Sommer-Kluß from the Rizzoli lab for being so helpful and patient.

I am really grateful, that I was part of the IMPRS Neuroscience graduate school. The wonderful diversity of people I met and the familial atmosphere have made me grow as a person. Especially, I want to thank Sandra Drube, Michael Hörner, Franziska Kühne, Steffen Burkhardt and Kirsten Pöhlker from the GGNB for their help and support. Your kindness and support allowed me to finish my work despite all the obstacles that had to be overcome, moving our lab to Gießen. Your help with the deadline extensions and the bridging fund have really saved my $\mathrm{PhD}$.

I want to thank the Central Mensa in Göttingen for the delicious meals they served for lunch. I cannot believe we ate that well for such a good price. Thank you to A. E. for always helping me with good advice regarding literature. I am very grateful to the RPG that has really made the transition from Göttingen to Gießen super easy... I want to particularly thank Innos who has always given me faith in science and has kept my fire of curiosity burning even in darker periods of my PhD. I want to thank the species Xenopus laevis for its sacrifice in the name of research. I hope my work will advance the knowledge in the field of neurobiology. Finally, I want to thank the uncaring, void universe for being so privileged to be able to fill almost two pages with gratitude for people in my life.

One must imagine Thomas Offner happy. 


\section{ABbreViations}

AMC: Accessory olfactory bulb mitral cell

ATC: Accessory tufted cell

ATP: Adenosine triphosphate

ADP: Adenosine diphosphate

AOB: Accessory olfactory bulb

AOS: Accessory olfactory system

cAMP: Cyclic adenosine monophosphate

DAG: Diacylglycerol

EPL: External plexiform layer

ETC: External tufted cell

GABA: Gamma amino butyric acid

GC: Granule cell

GCL: Granule cell layer

GDP: Guanosine diphosphate

GL: Glomerular layer

GPCR: G protein-coupled receptor

GTP: Guanosine triphosphate

JGC: Juxtaglomerular cell

LC: Lateral cluster

MC: Mitral cell

MCL: Mitral cell layer

MOB: Main olfactory bulb

MOE: Main olfactory epithelium

MOS: Main olfactory system

MRR: Molecular receptive range

MTC: Mitral/tufted cell

$\mathrm{NbT}$ : Neuronal beta tubulin

OB: Olfactory bulb

OlfC: Olfactory receptor class C-related

ON: Olfactory nerve

OR: OR type odorant receptor

ORA: Olfactory receptor class A-related

ORN: Olfactory receptor neuron

PGC: Periglomerular cell 
PLC: Phospholipase C

ROI: Region of interest

SSAC: superficial short axonal cell

TAAR: Trace-amine associated receptor

TC: Tufted cell

V1R: Vomeronasal type 1 receptor

V2R: Vomeronasal type 2 receptor

VR: Vomeronasal receptor

VRN: Vomeronasal receptor neuron

VNO: Vomeronasal organ

WGA: Wheat germ agglutinin

\section{REFERENCES}

Abraham, N.M., Egger, V., Shimshek, D.R., Renden, R., Fukunaga, I., Sprengel, R., Seeburg, P.H., Klugmann, M., Margrie, T.W., Schaefer, A.T., et al. (2010). Synaptic inhibition in the olfactory bulb accelerates odor discrimination in mice. Neuron 65, 399-411. Acher, F.C., and Bertrand, H.-O. (2005). Amino acid recognition by venus flytrap domains is encoded in an 8-residue motif. Biopolym. - Pept. Sci. Sect. 80, 357-366.

Ahuja, G., Ivandic, I., Saltürk, M., Oka, Y., Nadler, W., and Korsching, S.I. (2013). Zebrafish crypt neurons project to a single, identified mediodorsal glomerulus. Sci. Rep. 3, 2063.

Ahuja, G., Nia, S.B., Zapilko, V., Shiriagin, V., Kowatschew, D., Oka, Y., and Korsching, S.I. (2014). Kappe neurons, a novel population of olfactory sensory neurons. Sci. Rep. 4, 4037.

Alioto, T.S., and Ngai, J. (2005). The odorant receptor repertoire of teleost fish. BMC Genomics 6, 173.

Alioto, T.S., and Ngai, J. (2006). The repertoire of olfactory C family G protein-coupled receptors in zebrafish: candidate chemosensory receptors for amino acids. BMC Genomics 7, 309.

Allison, A.C. (1953). The structure of the olfactory bulb and its relationship to the olfactory pathways in the rabbit and the rat. J. Comp. Neurol. 98, 309-353.

Altner, H. (1962). Untersuchungen über Leistungen und Bau der Nase des südafrikanischen Krallenfrosches Xenopus laevis (Daudin 1803). Z Vergl. Physiol 45, $272-$ 306. 
Amjad, A., Hernandez-Clavijo, A., Pifferi, S., Maurya, D.K., Boccaccio, A., Franzot, J., Rock, J., and Menini, A. (2015). Conditional knockout of TMEM16A/anoctamin1 abolishes the calcium- activated chloride current in mouse vomeronasal sensory neurons. J. Gen. Physiol. 145, 285-301.

Aungst, J.L., Heyward, P.M., Puche, A.C., Karnup, S. V, Hayar, A., Szabo, G., and Shipley, M.T. (2003). Centre - surround inhibition among olfactory bulb glomeruli. Nature 426, 623.

Baier, H., and Korsching, S.I. (1994). Olfactory glomeruli in the zebrafish form an invariant pattern and are identifiable across animals. J. Neurosci. 14, 219-230.

Bains, G., Lee, R.T., Lee, Y.C., and Freire, E. (1992). Microcalorimetric study of wheat germ agglutinin binding to $\mathrm{N}$-acetylglucosamine and its oligomers. Biochemistry $31,12624-$ 12628.

Bakalyar, H.A., and Reed, R.R. (1990). Identification of a Specialized Adenylyl Cyclase That May Mediate Odorant Detection. Science (80-. ). 250, 1403-1406.

Baker, H., Cummings, D.M., Munger, S.D., Margolis, J.W., Franzen, L., Reed, R.R., and Margolis, F.L. (1999). Targeted Deletion of a Cyclic Nucleotide-Gated Channel Subunit ( OCNC1 ): Biochemical and Morphological Consequences in Adult Mice. J. Neurosci. 19, 9313-9321.

Ballesteros, J., and Palczewski, K. (2001). G protein-coupled receptor drug discovery: Implications from the crystal structure of rhodopsin. Curr Opin Drug Discov Devel 4, 561.

Banerjee, A., Marbach, F., Anselmi, F., Koh, M.S., Martin, B., Garcia, P., Delevich, K., Oyibo, H.K., Gupta, P., Li, B., et al. (2015). An interglomerular circuit gates glomerular output and implements gain control in the mouse olfactory bulb. Neuron 87, 193-207.

Barrios, A.W., Núñez, G., Sánchez Quinteiro, P., and Salazar, I. (2014). Anatomy, histochemistry, and immunohistochemistry of the olfactory subsystems in mice. Front. Neuroanat. 8, 63.

Barth, A.L., Dugas, J.C., and Ngai, J. (1997). Noncoordinate Expression of Odorant Receptor Genes Tightly Linked in the Zebrafish Genome. Neuron 19, 359-369.

Bayramli, X., Kocagöz, Y., Sakizli, U., and Fuss, S.H. (2017). Patterned arrangements of olfactory receptor gene expression in zebrafish are established by radial movement of specified olfactory sensory neurons. Sci. Rep. 7, 5572.

Bear, D.M., Jean-Marc, L., Hoekstra, H.E., and Datta, S.R. (2016). Evolution of the Genetic and Neural Architecture for Vertebrate Odor Perception. Curr. Biol. 26, R1039.

Belluscio, L., Gold, G.H., Nemes, A., and Axel, R. (1998). Mice deficient in G(olf) are anosmic. Neuron 20,69-81. 
Belluscio, L., Koentges, G., Axel, R., and Dulac, C. (1999). A map of pheromone receptor activation in the mammalian brain. Cell 97, 209-220.

Ben-shaul, Y., Katz, L.C., Mooney, R., and Dulac, C. (2010). In vivo vomeronasal stimulation reveals sensory encoding of conspecific and allospecific cues by the mouse accessory olfactory bulb. PNAS 107, 5172-5177.

Berridge, M.J. (1993). Inositol trisphosphate and calcium signalling. Nature 361, 315.

Bertrand, H.-O., Bessis, A.-S., Pin, J.-P., and Acher, F.C. (2002). Common and selective molecular determinants involved in metabotopic glutamate receptor agonist activity. J. Med. Chem. 45, 3171-3183.

Blanchart, A., De Carlos, J., and López-Mascaraque, L. (2006). Time frame of mitral cell development in mice olfactory bulb. J. Comp. Neurol. 469, 529-543.

Borowsky, B., Adham, N., Jones, K.A., Raddatz, R., Artymyshyn, R., Ogozalek, K.L., Durkin, M.M., Lakhlani, P.P., Bonini, J.A., Pathirana, S., et al. (2001). Trace amines: Identification of a family of mammalian G protein-coupled receptors. PNAS 98, 8966-8971.

Bozza, T., Feinstein, P., Zheng, C., and Mombaerts, P. (2002). Odorant receptor expression defines functional units in the mouse olfactory system. J. Neurosci. 22, 30333043.

Bozza, T., McGann, J.P., Mombaerts, P., and Wachowiak, M. (2004). In vivo imaging of neuronal activity by targeted expression of a genetically encoded probe in the mouse. Neuron 42, 9-21.

Braubach, O.R., Wood, H.-D., Gadbois, S., Fine, A., and Croll, R.P. (2009). Olfactory conditioning in the zebrafish (Danio rerio). Behav. Brain Res. 198, 190-198.

Braubach, O.R., Fine, A., and Croll, R.P. (2012). Distribution and functional organization of glomeruli in the olfactory bulbs of zebrafish (Danio rerio). J. Comp. Neurol. 520, 23172339.

Braubach, O.R., Miyasaka, N., Koide, T., Yoshihara, Y., Croll, R.P., and Fine, A. (2013). Experience-dependent versus experience-independent postembryonic development of distinct groups of zebrafish olfactory glomeruli. J. Neurosci. 33, 6905-6916.

Breer, H., Fleischer, J., and Strotmann, J. (2006). The sense of smell: Multiple olfactory subsystems. Cell Mol Life Sci 63, 1465-1475.

Brennan, P., and Keverne, E.B. (2015). Biological complexity and adaptability of simple mammalian olfactory memory systems. Neurosci. Biobehav. Rev. 50, 29-40.

Brinkmann, A., and Schild, D. (2016). One special glomerulus in the olfactory bulb of Xenopus laevis tadpoles integrates a broad range of amino acids and mechanical stimuli. J. 
Neurosci. 36, 10978-10989.

Broadwell, R.D., and Balin, B.J. (1985). Endocytic and exocytic pathways of the neuronal secretory process and trans synaptic transfer of wheat germ agglutinin-horseradish peroxidase in vivo. J. Comp. Neurol. 242, 632-650.

Brunet, L.J., Gold, G.H., and Ngai, J. (1996). General Anosmia Caused by a Targeted Disruption of the Mouse Olfactory Cyclic Nucleotide-Gated Cation Channel. Neuron 17, 681-693.

Buck, L.B., and Axel, R. (1991). A Novel Multigene Family May Encode Odorant Receptors: A Molecular Basis for Odor Recognition. Cell 65, 175-187.

Bulfone, A., Wang, F., Hevner, R., Anderson, S., Cutforth, T., Chen, S., Meneses, J., Pedersen, R., Axel, R., and Rubenstein, J.L.R. (1998). An olfactory sensory map develops in the absence of normal projection neurons or GABAergic interneurons. Neuron 21, 12731282.

Buonviso, N., Chaput, M.A., and Berthommier, F. (2002). Temporal pattern analyses in pairs of neighboring mitral cells. J. Neurophysiol. 68, 417-424.

Burton, S.D., and Urban, N.N. (2014). Greater excitability and firing irregularity of tufted cells underlies distinct afferent-evoked activity of olfactory bulb mitral and tufted cells. $\mathrm{J}$. Physiol. 592, 2097-2118.

Byrd, C.A., and Brunjes, P.C. (1995). Organization of the olfactory system in the adult zebrafish: Histological, immunohistochemical, and quantitative analysis. J. Comp. Neurol. $358,247-259$.

Cang, J., and Isaacson, J.S. (2003). In vivo whole-cell recording of odor-evoked synaptic transmission in the rat olfactory bulb. J. Neurosci. 23, 4108-4116.

Cao, Y., Oh, B.C., and Stryer, L. (1998). Cloning and localization of two multigene receptor families in goldfish olfactory epithelium. PNAS 95, 11987-11992.

Castro, J.B., and Urban, N.N. (2009). Subthreshold glutamate release from mitral cell dendrites. J. Neurosci. 29, 7023-7030.

Catania, K.C. (2013). Stereo and serial sniffing guide navigation to an odour source in a mammal. Nat. Commun. 4, 1441.

Cavarretta, F., Marasco, A., Hines, M.L., Shepherd, G.M., and Migliore, M. (2016).

Glomerular and mitral-granule cell microcircuits coordinate temporal and spatial information processing in the olfactory bulb. Front. Comput. Neurosci. 10, 67.

Cavarretta, F., Burton, S.D., Igarashi, K.M., Shepherd, G.M., Hines, M.L., and Migliore, M. (2018). Parallel odor processing by mitral and middle tufted cells in the olfactory bulb. Sci. 
Rep. 8, 7625 .

Cervino, A.S., Paz, D.A., and Frontera, J.L. (2017). Neuronal degeneration and regeneration induced by axotomy in the olfactory epithelium of Xenopus laevis. Dev. Neurobiol. 77, 1308-1320.

Chamero, P., Katsoulidou, V., Hendrix, P., Bufe, B., Roberts, R., Matsunami, H., Abramowitz, J., Birnbaumer, L., Zufall, F., and Leinders-Zufall, T. (2011). G protein Gao is essential for vomeronasal function and aggressive behavior in mice. PNAS 108, 1289812903.

Chamero, P., Leinders-Zufall, T., and Zufall, F. (2012). From genes to social communication: Molecular sensing by the vomeronasal organ. Trends Neurosci. 35, 597606.

Chan, L.L., Wilkinson, A.R., Paradis, B.D., and Lai, N. (2012). Rapid image-based cytometry for comparison of fluorescent viability staining methods. J Fluoresc $22,1301-$ 1311.

Charpak, S. (2001). Odor-evoked calcium signals in dendrites of rat mitral cells. PNAS 98, 1230-1234.

Chen, W.R., and Shepherd, G.M. (2005). The olfactory glomerulus: A cortical module with specific functions. J. Neurocytol. 34, 353-360.

Chen, C., Smye, S.W., Robinson, M.P., and Evans, J.A. (2006a). Membrane electroporation theories: A review. Medi Biol EngiComput 44, 5-14.

Chen, T.-W., Lin, B.-J., and Schild, D. (2009). Odor coding by modules of coherent mitral/tufted cells in the vertebrate olfactory bulb. PNAS 106, 2401-2406.

Chen, W.R., Shen, G.Y., Shepherd, G.M., Hines, M.L., and Midtgaard, J. (2006b). Multiple modes of action potential initiation and propagation in mitral cell primary dendrite. $\mathrm{J}$.

Neurophysiol. 88, 2755-2764.

Chess, A., Simon, I., Cedar, H., and Axel, R. (1994). Allelic inactivation regulates olfactory receptor gene expression. Cell 78, 823-834.

Christie, J.M., Bark, C., Hormuzdi, S.G., Helbig, I., Monyer, H., and Westbrook, G.L. (2005). Connexin36 mediates spike synchrony in olfactory bulb glomeruli. Neuron 46, 761-772.

Clapham, D.E. (2007). Calcium Signaling. Cell 131, 1047-1058.

Claxton, N.S., Fellers, T.J., and Davidson, M.W. (2006). Microscopy, confocal. Encycl. Med. Devices Instrum. 449-477.

Coffin, R.B. (1989). Bacterial uptake of dissolved free and combined amino acids in 
estuarine waters. Limnol. Ocean. 34, 531-542.

Cong, X., Qian, Z., Wenwen, R., Cheron, Jean-Baptiste, F.S., Wen, T., Zhang, C., Yu, H., Golebiowski, J., and Yu, Y. (2019). Zebrafish olfactory receptors ORAs differentially detect bile acids and bile salts. J. Biol. Chem. 294, 6762-6771.

Cox, J.P.L. (2008). Hydrodynamic aspects of fish olfaction. J. R. Soc. Interface 5, 575-593. Crick, F. (1988). What mad pursuit. Basic Books Inc, New York, 162.

Date-Ito, A., Ohara, H., Ichikawa, M., Mori, Y., and Hagino-Yamagishi, K. (2008). Xenopus V1R vomeronasal receptor family is expressed in the main olfactory system. Chem. Senses 33, 339-346.

DeMaria, S., Berke, A.P., Van Name, E., Heravian, A., Ferreira, T., and Ngai, J. (2013). Role of a ubiquitously expressed receptor in the vertebrate olfactory system. J. Neurosci. 33, 15235-15247.

Denk, W., and Svoboda, K. (1997). Photon Upmanship: Why multiphoton is more than a gimmick. Neuron 18, 351-357.

Denk, W., Strickler, J.H., and Webb, W.W. (1990). Two-Photon Laser Scanning Fluorescence Microscopy. Science (80-. ). 248, 73-76.

DeVries, S.H., and Baylor, D.A. (1993). Synaptic circuitry of the retina and olfactory bulb. Cell 72, 139-149.

Dhawale, A.K., Hagiwara, A., Bhalla, U.S., Murthy, V.N., and Albeanu, D.F. (2010). Nonredundant odor coding by sister mitral cells revealed by light addressable glomeruli in the mouse. Nat. Neurosci. 13, 1404.

Dibattista, M., Amjad, A., Maurya, D.K., Sagheddu, C., Montani, G., Tirindelli, R., and Menini, A. (2012). Calcium-activated chloride channels in the apical region of mouse vomeronasal sensory neurons. J. Gen. Physiol. 140, 3-15.

Dieris, M., Ahuja, G., Krishna, V., and Korsching, S.I. (2017). A single identified glomerulus in the zebrafish olfactory bulb carries the high-affinity response to death-associated odor cadaverine. Sci. Rep. 7, 40892.

Dittrich, K., Kuttler, J., Hassenklöver, T., and Manzini, I. (2016). Metamorphic remodeling of the olfactory organ of the African clawed frog, Xenopus laevis. J. Comp. Neurol. 524, 986998.

Dryer, L., and Graziadei, P.P.C. (1994). Mitral cell dendrites: a comparative approach. Anat. Embryol. (Berl). 189, 91-106.

Dulac, C., and Axel, R. (1995). A novel family of genes encoding putative pheromone 
receptors in mammals. Cell 83, 195-206.

Dulac, C., and Torello, A.T. (2003). Molecular detection of pheromone signals in mammals: From genes to behaviour. Nat. Rev. Neurosci. 4, 551.

Dynes, J.L., and Ngai, J. (1998). Pathfinding of olfactory neuron axons to stereotyped glomerular targets revealed by dynamic imaging in living zebrafish embryos. Neuron 20 , 1081-1091.

Economo, M.N., Hansen, K.R., and Wachowiak, M. (2016). Control of mitral/tufted Cell output by selective inhibition among olfactory bulb glomeruli. Neuron 91, 397-411.

Eerdunfu, Ihara, N., Ligao, B., Ikegaya, Y., and Takeuchi, H. (2017). Differential timing of neurogenesis underlies dorsal-ventral topographic projection of olfactory sensory neurons. Neural Dev. 12, 2.

Egaña, J.I., Aylwin, M.L., and Maldonado, P.E. (2005). Odor response properties of neighboring mitral/tufted cells in the rat olfactory bulb. Neuroscience 134, 1069-1080. Egger, V., and Urban, N.N. (2006). Dynamic connectivity in the mitral cell-granule cell microcircuit. Semin. Cell Dev. Biol. 17, 424-432.

Eisthen, H.L. (1997). Evolution of vertebrate olfactory systems. Brain. Behav. Evol. 50, 222-233.

Farrens, D.L., Altenbach, C., Yang, K., Hubbell, W.L., and Khorana, H.G. (1996). Requirement of rigid-body motion of transmembrane helices for light activation of rhodopsin. Science (80-. ). 274, 768-770.

Feinstein, P., and Mombaerts, P. (2004). A contextual model for axonal sorting into glomeruli in the mouse olfactory system. Cell 117, 817-831.

Feinstein, P., Bozza, T., Rodriguez, I., Vassalli, A., and Mombaerts, P. (2004). Axon guidance of mouse olfactory sensory neurons by odorant receptors and the $\beta 2$ adrenergic receptor. Cell 117, 833-846.

Figueres-Oñate, M., Gutiérrez, Y., and López-Mascaraque, L. (2014). Unraveling Cajal's view of the olfactory system. Front. Neuroanat. 8, 55.

Firestein, S., Darrow, B., and Shepherd, G.M. (1991). Activation of the sensory current in salamander olfactory receptor neurons depends on a G protein-mediated cAMP second messenger system. Neuron 6, 825-835.

Freitag, J., Krieger, J., Strotmann, J., and Breer, H. (1995). Two classes of olfactory receptors in Xenopus laevis. Neuron 15, 1383-1392.

Freitag, J., Beck, A., Ludwig, G., Von Buchholtz, L., and Breer, H. (1999). On the origin of 
the olfactory receptor family: receptor genes of the jawless fish (Lampetra fluviatilis). Gene 226, 165-174.

Fried, H.U., Fuss, S.H., and Korsching, S.I. (2002). Selective imaging of presynaptic activity in the mouse olfactory bulb shows concentration and structure dependence of odor responses in identified glomeruli. PNAS 99, 3222-3227.

Friedrich, R.W., and Korsching, S.I. (1997). Combinatorial and chemotopic odorant coding in the zebrafish olfactory bulb visualized by optical imaging. Neuron 18, 737-752.

Friedrich, R.W., and Korsching, S.I. (1998). Chemotopic, combinatorial, and noncombinatorial odorant representations in the olfactory bulb revealed using a voltagesensitive axon tracer. J. Neurosci. 18, 9977-9988.

Friedrich, J., Zhou, P., and Paninski, L. (2017). Fast online deconvolution of calcium imaging data. PLoS Comput. Biol. 13, e1005423.

Fukunaga, I., Herb, J., Kollo, M., Boyden, E.S., and Andreas, T. (2014). Independent control of gamma and theta activity by distinct interneuron networks in the olfactory bulb. Nat. Neurosci. 17, 1208.

Fuller, C.L., Yettaw, H.K., and Byrd, C.A. (2006). Mitral cells in the olfactory bulb of adult zebrafish (Danio rerio): morphology and distribution. J. Comp. Neurol. 499, 218-230.

Garaschuk, O., Milos, R.-I., and Konnerth, A. (2006). Targeted bulk-loading of fluorescent indicators for two-photon brain imaging in vivo. Nat. Protoc. 1, 380.

Gaudin, A., and Gascuel, J. (2005). 3D atlas describing the ontogenic evolution of the primary olfactory projections in the olfactory bulb of Xenopus laevis. J. Comp. Neurol. 489, 403-424.

Gautam, S.H., and Verhagen, J. V. (2012). Retronasal odor representations in the dorsal olfactory bulb of rats. J. Neurosci. 32, 7949-7959.

Geramita, M., and Urban, N.N. (2017). Differences in glomerular-layer-mediated feedforward inhibition onto mitral and tufted cells lead to distinct modes of intensity coding. J. Neurosci. 37, 1428-1438.

Ghosh, S., Larson, S.D., Hefzi, H., Marnoy, Z., Cutforth, T., Dokka, K., and Baldwin, K.K. (2011). Sensory maps in the olfactory cortex defined by long-range viral tracing of single neurons. Nature 472, 217.

Giorgi, C., Danese, A., Missiroli, S., Patergnani, S., and Pinton, P. (2018). Calcium Dynamics as a Machine for Decoding Signals. Trends Cell Biol. 28, 258-273.

Giovannucci, A., Friedrich, J., Gunn, P., Kalfon, J., Taxidis, J., Naja, F., Gauthier, J., Tank, D., Giovannucci, A., Friedrich, J., et al. (2019). CalmAn: An open source tool for scalable 
calcium imaging data analysis. Elife 8, e38173.

Gire, D.H., Franks, K., Zak, J.D., Tanaka, K.F., Whitesell, J., Mulligan, A.A., Hen, R., and Schoppa, N.E. (2012). Mitral cells in the olfactory bulb are mainly excited through a multistep signaling path. J. Neurosci. 32, 2964-2975.

Gliem, S., Syed, A.S., Sansone, A., Kludt, E., Tantalaki, E., Hassenklöver, T., Korsching, S.I., and Manzini, I. (2013). Bimodal processing of olfactory information in an amphibian nose: odor responses segregate into a medial and a lateral stream. Cell. Mol. Life Sci. 70, 1965-1984.

Göbel, W., and Helmchen, F. (2012). In vivo calcium imaging of neural network function in vivo calcium imaging of neural network function. Physiology 22, 358-365.

Gonatas, N.K., Harper, C., Mizutani, T., and Gonatas, J.O. (1979). Superior sensitivity of conjugates of horseradish peroxidase with wheat germ agglutinin for studies of retrograde axonal transport. J. Histochem. Cytochem. 27, 728-734.

González, A., Tuinhof, R., and Smeets, W.J.A.J. (1993). Distribution of tyrosine hydroxylase and dopamine immunoreactivities in the brain of the South African clawed frog Xenopus laevis. Anat. Embryol. (Berl). 187, 193-201.

Göppert-Mayer, M. Elementary processes with two quantum transitions. Ann. Der Phys. 18, 466-479.

Gorin, M., Tsitoura, C., Kahan, A., Watznauer, K., Drose, D.R., Arts, M., Mathar, R., O'Connor, S., Hanganu-Opatz, I.L., Ben-Shaul, Y., et al. (2016). Interdependent conductances drive infraslow intrinsic rhythmogenesis in a subset of accessory olfactory bulb projection neurons. J. Neurosci. 36, 3127-3144.

Greer, P.L., Bear, D.M., Lassance, J.M., Bloom, M.L., Tsukahara, T., Pashkovski, S.L., Masuda, F.K., Nowlan, A.C., Kirchner, R., Hoekstra, H.E., et al. (2016). A family of nonGPCR chemosensors defines an alternative logic for mammalian olfaction. Cell 165, 17341748.

Grüneberg, H. (1973). A ganglion probably belonging to the N. terminalis system in the nasal mucosa of the mouse. Z. Anat. Entwicklungsgesch. 140, 39-52.

Gschwend, O., Abraham, N.M., Lagier, S., Begnaud, F., Rodriguez, I., and Carleton, A. (2015). Neuronal pattern separation in the olfactory bulb improves odor discrimination learning. Nat. Neurosci. 18, 1474.

Gutièrrez-Mecinas, M., Crespo, C., Blasco-Ibáñez, J.M., Gracia-Llanes, F.J., Marqués-Marí, A.I., and Martínez-Guijarro, F.J. (2005). Characterization of somatostatin- and cholecystokinin-immunoreactive periglomerular cells in the rat olfactory bulb. J. Comp. Neurol. 489, 467-479. 
Haas, K., Sin, W., Javaherian, A., Li, Z., and Cline, H.T. (2001). for Gene Transfer In Vivo. Neuron 29, 583-591.

Haas, K., Jensen, K., Sin, W.C., Foa, L., and Cline, H.T. (2002). Targeted electroporation in Xenopus tadpoles in vivo-from single cells to the entire brain. Differentiation 70, 148-154.

Hagino-Yamagishi, K., Moriya, K., Kubo, H., Wakabayashi, Y., Isobe, N., Saito, S., Ichikawa, M., and Yazaki, K. (2004). Expression of vomeronasal receptor genes in Xenopus laevis. J. Comp. Neurol. 472, 246-256.

Halpern, M., and Martínez-Marcos, A. (2003). Structure and function of the vomeronasal system: an update. Prog. Neurobiol. 70, 245-318.

Halpern, M., Shapiro, L.S., and Jia, C. (1995). Differential localization of G proteins in the opossum vomeronasal system. Brain Res. 677, 157-161.

Hamdani, E.H., and Døving, K.B. (2002). The alarm reaction in crucian carp is mediated by olfactory neurons with long dendrites. Chem. Senses $27,395-398$.

Hamdani, E.H., and Døving, K.B. (2007). The functional organization of the fish olfactory system. Prog. Neurobiol. 82, 80-86.

Hamdani, E.H., Alexander, G., and Døving, K.B. (2001). Projection of sensory neurons with microvilli to the lateral olfactory tract indicates their participation in feeding behaviour in crucian carp. Chem. Senses 26, 1139-1144.

Hammen, G.F., Turaga, D., Holy, T.E., and Meeks, J.P. (2014). Functional organization of glomerular maps in the mouse accessory olfactory bulb. Nat. Neurosci. 17, 953.

Hansen, A., and Finger, T.E. (2000). Phyletic distribution of crypt-type olfactory receptor neurons in fishes. Brain. Behav. Evol. 55, 100-110.

Hansen, A., Reiss, J.O., Gentry, C.L., and Burd, G.D. (1998). Ultrastructure of the olfactory organ in the clawed frog, Xenopus laevis, during larval development and metamorphosis. J. Comp. Neurol. 398, 273-288.

Hansen, A., Rolen, S.H., Anderson, K., Morita, Y., Caprio, J., and Finger, T.E. (2003). Correlation between olfactory receptor cell type and function in the channel catfish. $\mathrm{J}$. Neurosci. 23, 9328-9339.

Hansen, A., Anderson, K.T., and Finger, T.E. (2004). Differential distribution of olfactory receptor neurons in goldfish: structural and molecular correlates. J. Comp. Neurol. 477, 347-359.

Hasegawa, S., Hirabayashi, T., Kondo, T., Inoue, K., Esumi, S., Okayama, A., Hamada, S., and Yagi, T. (2012). Constitutively expressed Protocadherin- $\alpha$ regulates the coalescence and elimination of homotypic olfactory axons through its cytoplasmic region. Front. Mol. 
Neurosci. 5, 97.

Hashiguchi, Y., and Nishida, M. (2007). Evolution of trace amine-associated receptor (TAAR) gene family in vertebrates: lineage-specific expansions and degradations of a second class of vertebrate chemosensory receptors expressed in the olfactory epithelium. Mol. Biol. Evol. 24, 2099-2107.

Hassenklöver, T., and Manzini, I. (2013). Olfactory wiring logic in amphibians challenges the basic assumptions of the unbranched axon concept. J. Neurosci. 33, 17247-17252.

Hassenklöver, T., Pallesen, L.P., Schild, D., and Manzini, I. (2012). Amino acid- vs. peptideodorants: responses of individual olfactory receptor neurons in an aquatic species. PLoS One 7, e53097.

Hawkins, S.J., Weiss, L., Offner, T., Dittrich, K., Hassenklöver, T., and Manzini, I. (2017). Functional reintegration of sensory neurons and transitional dendritic reduction of mitral/tufted cells during injury-induced recovery of the larval Xenopus olfactory circuit. Front. Cell. Neurosci. 11, 380.

Hayar, A., Karnup, S., Ennis, M., and Shipley, M.T. (2004a). External tufted cells: a major excitatory element that coordinates glomerular activity. J. Neurosci. 24, 6676-6685.

Hayar, A., Karnup, S., Shipley, M.T., and Ennis, M. (2004b). Olfactory bulb glomeruli: external tufted cells intrinsically burst at theta frequency and are entrained by patterned olfactory input. J. Neurosci. 24, 1190-1199.

Hayar, A., Shipley, M.T., and Ennis, M. (2005). Olfactory bulb external tufted cells are synchronized by multiple intraglomerular mechanisms. J. Neurosci. 25, 8197-8208.

Hayashi, Y., Momiyama, A., Takahashi, T., Ohishi, H., Ogawa-Meguro, R., Shigemoto, R., Mizuno, N., and Nakanishi, S. (1993). Role of metabotropic glutamate receptor in synaptic modulation of the accessory olfactory bulb. Nature 366, 687 .

Helmchen, F., and Denk, W. (2005). Deep tissue two-photon microscopy. Nat. Methods 2, 932.

Herrada, G., and Dulac, C. (1997). A novel family of putative pheromone receptors in mammals with a topographically organized and sexually dimorphic distribution. Cell 90 , 763-773.

Hildebrand, J.G., and Shepherd, G.M. (1997). Mechanisms of olfactory discrimination: converging evidence for common principles across phyla. Annu. Rev. Neurosci. 20, 595631.

Ho, S.Y., and Mittal, G.S. (1996). Electroporation of Cell Membranes : A Review. Crit. Rev. Biotechnol. 16, 349-362. 
Holy, T.E. (2000). Responses of vomeronasal neurons to natural stimuli. Sci. Reports 289, 1569-1572.

Homma, R., Kovalchuk, Y., Konnerth, A., Cohen, L.B., and Garaschuk, O. (2013). In vivo functional properties of juxtaglomerular neurons in the mouse olfactory bulb. Front. Neural Circuits 7, 23.

Homma, R., Lv, X., Sato, T., Imamura, F., Zeng, S., and Nagayama, S. (2019). Narrowly confined and glomerulus-specific onset latencies of odor-evoked calcium transients in the juxtaglomerular cells of the mouse main olfactory bulb. ENeuro 6, e0387-18.

Hubel, D.H., and Wiesel, T.N. (1959). Receptive fields of single neurones in the cat's striate cortex. J. Physiol. 148, 574-591.

Hunter, J.D. (2007). Matplotlib: A 2D graphics environment. Comput. Sci. Eng. 9, 90.

Hussain, A., Saraiva, L.R., and Korsching, S.I. (2009). Positive Darwinian selection and the birth of an olfactory receptor clade in teleosts. PNAS 106, 4313-4318.

Igarashi, K.M., and Mori, K. (2004). Spatial representation of hydrocarbon odorants in the ventrolateral zones of the rat olfactory bulb. J. Neurophysiol. 93, 1007-1019.

Igarashi, K.M., leki, N., An, M., Yamaguchi, Y., Nagayama, S., Kobayakawa, K., Kobayakawa, R., Tanifuji, M., Sakano, H., Chen, W.R., et al. (2012). Parallel mitral and tufted cell pathways route distinct odor information to different targets in the olfactory cortex. J. Neurosci. 32, 7970-7985.

Imai, T., and Sakano, H. (2007). Roles of odorant receptors in projecting axons in the mouse olfactory system. Curr. Opin. Neurobiol. 17, 507-515.

Imai, T., Yamazaki, T., Kobayakawa, R., Kobayakawa, K., Abe, T., Suzuki, M., and Sakano, H. (2009). Pre-target axon sorting establishes the neural map topography. Science (80-. ). $325,585-590$.

Isaacson, J.S. (1999). Glutamate spillover mediates excitatory transmission in the rat olfactory bulb. Neuron 23, 377-384.

Isaacson, J.S., and Strowbridge, B.W. (1998). Olfactory reciprocal synapses: dendritic signaling in the CNS. Neuron 20, 749-761.

Ishii, T., and Mombaerts, P. (2011). Coordinated coexpression of two vomeronasal receptor V2R genes per neuron in the mouse. Mol. Cell. Neurosci. 46, 397-408.

Isogai, Y., Si, S., Pont-Lezica, L., Tan, T., Kapoor, V., Murthy, V.N., and Dulac, C. (2012). Molecular organization of vomeronasal chemoreception. Nature 478, 241.

Jablonski, A. (1933). Efficiency of Anti-Stokes Fluorescence in Dyes. Nature 131, 839. 
Jahr, C.E., and Nicoll, R.A. (1981). Primary afferent depolarization in the in vitro frog olfactory bulb. J. Physiol. 318, 375-384.

Jia, C., Chen, W.R., and Shepherd, G.M. (1999). Synaptic organization and neurotransmitters in the rat accessory olfactory bulb. J. Neurophysiol. 81, 345-354.

Jiang, T., and Holley, A. (1992). Morphological variations among output neurons of the olfactory bulb in the frog (Rana ridibunda). J. Comp. Neurol. 320, 86-96.

Jones, D.T., and Reed, R.R. (1989). Golf: an olfactory neuron specific-G protein involved in odorant signal transduction. Science (80-. ). 244, 790-795.

Jørgens, N.G. (1986). Fluxes of free amino acids in three Danish lakes. Freshw. Biol. 16, 255-268.

Junek, S., Kludt, E., Wolf, F., and Schild, D. (2010). Olfactory coding with patterns of response latencies. Neuron 67, 872-884.

Kaba, H., Hayashi, Y., Higuchi, T., and Nakanishi, S. (1994). Induction of an olfactory memory by the activation of a metabotropic glutamate receptor. Science (80-. ). 265, 262264.

Kahan, A., and Ben-shaul, Y. (2016). Extracting behaviorally relevant traits from natural stimuli : benefits of combinatorial representations at the accessory olfactory bulb. PLoS Comput. Biol. 12, e1004798.

Katada, S., Hirokawa, T., Oka, Y., Suwa, M., and Touhara, K. (2005). Structural basis for a broad but selective ligand spectrum of a mouse olfactory receptor: mapping the odorantbinding site. J. Neurosci. 25, 1806-1815.

Kato, A., Katada, S., and Touhara, K. (2008). Amino acids involved in conformational dynamics and G-protein coupling of an odorant receptor: Targeting gain-of-function mutation. J. Neurochem. 107, 1261-1270.

Katz, P.S., and Calin-Jageman, R.J. (2009). Neuromodulation.

Katz, P.S., and Frost, W.N. (1996). Intrinsic neuromodulation: altering neuronal circuits from within. Trends Neurosci. 19, 54-61.

Kay, L.M. (2015). Olfactory system oscillations across phyla. Curr. Opin. Neurobiol. 31, 141-147.

Kermen, F., Franco, L.M., Wyatt, C., and Yaksi, E. (2013). Neural circuits mediating olfactory-driven behavior in fish. Front. Neural Circuits 7, 62.

Kikuta, S., Fletcher, M.L., Homma, R., Yamasoba, T., and Nagayama, S. (2013). Odorant response properties of individual neurons in an olfactory glomerular module. Neuron 77 , 
$1122-1135$.

Kim, S., Ma, L., and Yu, C.R. (2011). Requirement of calcium-activated chloride channels in the activation of mouse vomeronasal neurons. Nat. Commun. 2, 365.

Kim, S., Ma, L., Unruh, J., Mckinney, S., and Yu, C.R. (2015). Intracellular chloride concentration of the mouse vomeronasal neuron. BMC Neurosci. 16, 90.

Kimoto, H., Haga, S., Sato, K., and Touhara, K. (2005). Sex-specific peptides from exocrine glands stimulate mouse vomeronasal sensory neurons. Nature 437, 898.

Kleene, S.J., and Gesteland, R.C. (1991). Calcium-activated chloride conductance in frog olfactory cilia. J. Neurosci. 11, 3624-3629.

Klein, S.L., and Graziadei, P.P.C. (1983). The differentiation of the olfactory placode in Xenopus laevis: A light and electron microscope study. J. Comp. Neurol. 217, 17-30.

Klenoff, J.R., and Greer, C.A. (1998). Postnatal development of olfactory receptor cell axonal arbors. J. Comp. Neurol. 390, 256-267.

Kludt, E., Okom, C., Brinkmann, A., and Schild, D. (2015). Integrating temperature with odor processing in the olfactory bulb. J. Neurosci. 35, 7892-7902.

Koide, T., Miyasaka, N., Morimoto, K., Asakawa, K., Urasaki, A., Kawakami, K., and Yoshihara, Y. (2009). Olfactory neural circuitry for attraction to amino acids revealed by transposon-mediated gene trap approach in zebrafish. PNAS 106, 9884-9889.

Korsching, S.I. (2001). Odor maps in the brain: Spatial aspects of odor representation in sensory surface and olfactory bulb. Cell. Mol. Life Sci. 58, 520-530.

Korsching, S.I. (2002). Olfactory maps and odor images. Curr. Opin. Neurobiol. 12, 387392.

Korsching, S.I. (2008). The molecular evolution of teleost olfactory receptor gene families. Chemosens. Syst. Mammals, Fishes, Insects 221-238.

Kosaka, K., and Kosaka, T. (2005). Synaptic organization of the glomerulus in the main olfactory bulb: Compartments of the glomerulus and heterogeneity of the periglomerular cells. Anat. Sci. Int. 80, 80-90.

Kosaka, T., and Hama, K. (1982). Structure of the mitral cell in the olfactory bulb of the goldfish (Carassius auratus). J. Comp. Neurol. 212, 365-384.

Kosaka, T., and Kosaka, K. (2016). Neuronal organization of the main olfactory bulb revisited. Anat. Sci. Int. 91, 115-127.

Kosaka, K., Toida, K., Aika, Y., and Kosaka, T. (1998). How simple is the organization of the olfactory glomerulus?: The heterogeneity of so-called periglomerular cells. Neurosci. 
Res. 30, 101-110.

Kristensson, K., and Olsson, Y. (1971). Retrograde axonal transport of protein. Brain Res. $29,363-365$.

Kuffler, S.W. (1953). Discharge patterns and functional organization of mammalian retina. J. Neurophysiol. 16, 37-68.

Kupfermann, I. (1979). Modulatory actions of neurotransmitters. Annu. Rev. Neurosci. 2, 447-465.

Kurahashi, T., and Yau, K.-W. (1993). Co-existence of cationic and chloride components in odorant induced current of vertebrate olfactory receptor cells. Nature 363, 71-74.

Lancet, D., Greer, C. a, Kauer, J.S., and Shepherd, G.M. (1982). Mapping of odor-related neuronal activity in the olfactory bulb by high-resolution 2-deoxyglucose autoradiography. PNAS 79, 670-674.

Larriva-Sahd, J. (2008). The accessory olfactory bulb in the adult rat: A cytological study of its cell types, neuropil, neuronal modules, and interactions with the main olfactory system. J. Comp. Neurol. 510, 309-350.

Laurent, G., Stopfer, M., Friedrich, R.W., Rabinovich, M.I., Volkovskii, A., and Abarbanel, H.D.I. (2001). Odor encoding as an active, dynamical process: Experiments, computation, and theory. Annu. Rev. Neurosci. 24, 263-297.

LaVail, J.H., and LaVail, M.M. (1972). Retrograde axonal transport in the central nervous system. Science (80-. ). 176, 1416-1417.

Le, Y., Murphy, P.M., and Wang, J.M. (2002). Formyl-peptide receptors revisited. Trends Immunol. 23, 541-548.

LeCluyse, E.L., Frost, S.K., and Dentler, W.L. (1985). Development and ciliation of the palate in two frogs , Bombina and Xenopus ; A comparative study. Tissue Cell 17, 853-864.

Leinders-Zufall, T., Brennan, P., Widmayer, P., Chandramani S., P., Maul-Pavicic, A., Jäger, M., Li, X.H., Breer, H., Zufall, F., and Boehm, T. (2004). MHC class I peptides as chemosensory signals in the vomeronasal organ. Science (80-. ). 306, 1033-1037.

Leinders-Zufall, T., Ishii, T., Mombaerts, P., Zufall, F., and Boehm, T. (2009). Structural requirements for the activation of vomeronasal sensory neurons by MHC peptides. Nat. Neurosci. 12, 1551.

Lévai, O., Feistel, T., Breer, H., and Strotmann, J. (2006). Cells in the vomeronasal organ express odorant receptors but project to the accessory bulb. J. Comp. Neurol. 498, 476490. 
Li, Q., Tachie-Baffour, Y., Liu, Z., Baldwin, M.W., Kruse, A.C., and Liberles, S.D. (2015). Non-classical amine recognition evolved in a large clade of olfactory receptors. Elife 4 , e10441.

Liberles, S.D., and Buck, L.B. (2006). A second class of chemosensory receptors in the olfactory epithelium. Nature $442,645$.

Liberles, S.D., Horowitz, L.F., Kuang, D., Contos, J.J., Wilson, K.L., Siltberg-Liberles, J., Liberles, D.A., and Buck, L.B. (2009). Formyl peptide receptors are candidate chemosensory receptors in the vomeronasal organ. PNAS 106, 9842-9847.

Lichtman, J.W., and Conchello, J. (2005). Fluorescence microscopy. Nat. Methods 2, 910919.

Liman, E.R., Corey, D.P., and Dulac, C. (1999). TRP2 : A candidate transduction channel for mammalian pheromone sensory signaling. PNAS 96, 5791-5796.

Lin, M.Z., and Schnitzer, M. (2016). Genetically encoded indicators of neuronal activity. Nat. Neurosci. 19, 1142.

Lin, B.J., Chen, T.W., and Schild, D. (2007). Cell type-specific relationships between spiking and $[\mathrm{Ca} 2+]$ in neurons of the Xenopus tadpole olfactory bulb. J. Physiol. 582, 163175.

Lin, D.M., Wang, F., Lowe, G., Gold, G.H., Axel, R., Ngai, J., and Brunet, L. (2000). Formation of precise connections in the olfactory bulb occurs in the absence of odorantevoked neuronal activity. Neuron 26, 69-80.

Lindemann, L., and Hoener, M.C. (2005). A renaissance in trace amines inspired by a novel GPCR family. Trends Pharmacol. Sci. 26, 274-281.

Lindemann, L., Ebeling, M., Kratochwil, N.A., Bunzow, J.R., Grandy, D.K., and Hoener, M.C. (2005). Trace amine-associated receptors form structurally and functionally distinct subfamilies of novel G protein-coupled receptors. Genomics 85, 372-385.

Lizbinski, K.M., and Dacks, A.M. (2018). Intrinsic and extrinsic neuromodulation of olfactory processing. Front. Cell. Neurosci. 11, 424.

López-Mascaraque, L., and de Castro, F. (2002). The olfactory bulb as an independent developmental domain. Cell Death Differ. 9, 1279-1286.

López-Mascaraque, L., De Carlos, J.A., and Valverde, F. (1996). Early onset of the rat olfactory bulb projections. Neuroscience 70, 255-266.

Lowe, G., and Gold, G.H. (1993). Nonlinear amplification by calcium-dependent chloride channels in olfactory receptor cells. Nature 366, 283. 
Lucas, P., Ukhanov, K., Leinders-zufall, T., and Zufall, F. (2003). A diacylglycerol-gated cation channel in vomeronasal neuron dendrites is impaired in TRPC2 mutant mice: Mechanism of pheromone transduction. Neuron 40, 551-561.

Luo, L., Callaway, E.M., and Svoboda, K. (2008). Genetic dissection of neural circuits. Neuron 57, 634-660.

Ma, J., and Lowe, G. (2004). Action potential backpropagation and multiglomerular signaling in the rat vomeronasal system. J. Neurosci. 24, 9341-9352.

Ma, J., and Lowe, G. (2010). Correlated firing in tufted cells of mouse olfactory bulb. Neuroscience 169, 1715-1738.

Macrides, F., and Schneider, S.P. (1982). Laminar organization of mitral and tufted cells in the main olfactory bulb of the adult hamster. J. Comp. Neurol. 208, 419-430.

Malnic, B., Hirono, J., Sato, T., and Buck, L.B. (1999). Combinatorial receptor codes for odors. Cell 96, 713-723.

Manzini, I., and Korsching, S. (2011). The peripheral olfactory system of vertebrates: molecular, structural and functional basics of the sense of smell. E-Neuroforum 17, 68-77. Manzini, I., and Schild, D. (2003a). cAMP-independent olfactory transduction of amino acids in Xenopus laevis tadpoles. J. Physiol. 551, 115-123.

Manzini, I., and Schild, D. (2003b). Multidrug resistance transporters in the olfactory receptor neurons of Xenopus laevis tadpoles. J. Physiol. 546, 375-385.

Manzini, I., and Schild, D. (2004). Classes and narrowing selectivity of olfactory receptor neurons of Xenopus laevis tadpoles. J. Gen. Physiol. 123, 99-107.

Manzini, I., Peters, F., and Schild, D. (2002). Odorant responses of Xenopus laevis tadpole olfactory neurons: A comparison between preparations. J. Neurosci. Methods 121, 159167.

Manzini, I., Heermann, S., Czesnik, D., Brase, C., Schild, D., and Rössler, W. (2007a). Presynaptic protein distribution and odour mapping in glomeruli of the olfactory bulb of Xenopus laevis tadpoles. Eur. J. Neurosci. 26, 925-934.

Manzini, I., Brase, C., Chen, T.-W., and Schild, D. (2007b). Response profiles to amino acid odorants of olfactory glomeruli in larval Xenopus laevis. J. Physiol. 581, 567-579.

Margrie, T.W., and Schaefer, A.T. (2003). Theta oscillation coupled spike latencies yield computational vigour in a mammalian sensory system. J. Physiol. 546, 363-374.

Matsumoto, H., Kobayakawa, K., Kobayakawa, R., Tashiro, T., Mori, K., Sakano, H., and Mori, K. (2010). Spatial arrangement of glomerular molecular-feature clusters in the 
odorant-receptor class domains of the mouse olfactory bulb. J. Neurophysiol. 103, 34903500 .

Matsunami, H., and Buck, L.B. (1997). A multigene family encoding a diverse array of putative pheromone receptors in mammals. Cell 90, 775-784.

Meeks, J.P., Arnson, H.A., and Holy, T.E. (2010). Representation and transformation of sensory information in the mouse accessory olfactory system. Nat. Neurosci. 13, 723.

Meisami, E. (1989). A proposed relationship between increases in the number of olfactory receptor neurons, convergence ratio and sensitivity in the developing rat. Dev Brain Res 46, 9-19.

Meisami, E. (1991). Convergence in the vomeronasal and main olfactory system of neonatal and mature mammals and its relation to sensitivity. Soc Neurosci Abstr 17, 635.

Meisami, E., and Bhatnagar, K.P. (1998). Structure and diversity in mammalian accessory olfactory bulb. Microsc. Res. Tech. 43, 476-499.

Meister, M., and Bonhoeffer, T. (2001). Tuning and topography in an odor map on the rat olfactory bulb. J. Neurosci. 21, 1351-1360.

Meredith, M. (1991). Sensory processing in the main and accessory olfactory systems: comparisons and contrasts. J Steroid Biochem Mol Biol 39, 601-614.

Mezler, M., Konzelmann, S., Freitag, J., Rössler, P., and Breer, H. (1999). Expression of olfactory receptors during development in Xenopus laevis. J. Exp. Biol. 202, 365-376.

Miyamichi, K., Serizawa, S., and Sakano, H. (2005). Continuous and overlapping expression domains of odorant receptor genes in the olfactory epithelium determine the dorsal/ventral positioning of glomeruli in the olfactory bulb. J. Neurosci. 25, 3586-3592.

Mohrhardt, J., Nagel, M., Fleck, D., Ben-Shaul, Y., and Spehr, M. (2018). Signal detection and coding in the accessory olfactory system. Chem. Senses 43, 667-695.

Mombaerts, P. (1996). Targeting olfaction. Curr. Opin. Neurobiol. 6, 481-486.

Mombaerts, P. (2004a). Genes and ligands for odorant, vomeronasal and taste receptors. Nat. Rev. Neurosci. 5, 263.

Mombaerts, P. (2004b). Odorant receptor gene choice in olfactory sensory neurons: The one receptor-one neuron hypothesis revisited. Curr. Opin. Neurobiol. 14, 31-36.

Mombaerts, P. (2006). Axonal wiring in the mouse olfactory system. Annu. Rev. Cell Dev. Biol. 22, 713-737.

Mombaerts, P., Wang, F., Dulac, C., Chao, S.K., Nemes, A., Mendelsohn, M., Edmondson, J., and Axel, R. (1996). Visualizing an olfactory sensory map. Cell 87, 675-686. 
Moody, S.A., Miller, V., Spanos, A., and Frankfurter, A. (1996). Developmental expression of a neuron-specific beta-tubulin in frog (Xenopus laevis): A marker for growing axons during the embryonic period. J. Comp. Neurol. 364, 219-230.

Mori, K. (1987). Membrane and synaptic properties of identified neurons in the olfactory bulb. Prog Neurobiol 29, 275-320.

Mori, K., and Sakano, H. (2011). How is the olfactory map formed and interpreted in the mammalian brain? Annu. Rev. Neurosci. 34, 467-499.

Mori, K., and Shepherd, G.M. (1994). Emerging principles of molecular signal processing by mitral/tufted cells in the olfactory bulb. Semin. Cell Biol. 5, 65-74.

Mori, K., Kishi, K., and Ojima, H. (1983). Distribution of dendrites of mitral, displaced mitral, tufted, and granule cells in the rabbit olfactory bulb. J. Comp. Neurol. 219, 339-355.

Mori, K., von Campenhausen, H., and Yoshihara, Y. (2000). Zonal organization of the mammalian main and accessory olfactory systems. Philos. Trans. R. Soc. Lond. B. Biol. Sci. 355, 1801-1812.

Mori, K., Takahashi, Y.K., Igarashi, K.M., and Yamaguchi, M. (2006). Maps of odorant molecular features in the mammalian olfactory bulb. Physiol. Rev. 86, 409-433.

Morita, Y., and Finger, T.E. (1998). Differential projections of ciliated and microvillous olfactory receptor cells in the catfish, Ictalurus punctatus. J. Comp. Neurol. 398, 539-550.

Moriya-Ito, K., Endoh, K., Fujiwara-Tsukamoto, Y., and Ichikawa, M. (2013). Threedimensional reconstruction of electron micrographs reveals intrabulbar circuit differences between accessory and main olfactory bulbs. Front. Neuroanat. 7, 5.

Morona, R., and González, A. (2013). Pattern of calbindin-D28k and calretinin immunoreactivity in the brain of Xenopus laevis during embryonic and larval development. J. Comp. Neurol. 521, 79-108.

Mugnaini, E., Oertel, W.H., and Wouterlood, F.F. (1984). Immunocytochemical Localization of GABA neurons and dopamine neurons in the rat main and accessory bulbs. Neurosci. Lett. 47, 221-226.

Münch, J., Billig, G., Hübner, C.A., Leinders-Zufall, T., Zufall, F., and Jentsch, T.J. (2018). $\mathrm{Ca} 2+$-activated $\mathrm{Cl}$ - currents in the murine vomeronasal organ enhance neuronal spiking but are dispensable for male-male aggression aggression. JBC 293, 10392-10403.

Munger, S.D., Leinders-Zufall, T., and Zufall, F. (2009). Subsystem organization of the mammalian sense of smell. Annu. Rev. Physiol. 71, 115-140.

Nagao, H., Yoshihara, Y., Mitsui, S., Fujisawa, H., and Mori, K. (2000). Two mirror-image sensory maps with domain organization in the mouse main olfactory bulb. Neuroreport 11 , 
Nagayama, S. (2010). Differential axonal projection of mitral and tufted cells in the mouse main olfactory system. Front. Neural Circuits 4, 120.

Nagayama, S., Takahashi, Y.K., Yoshihara, Y., and Mori, K. (2004). Mitral and tufted cells differ in the decoding manner of odor maps in the rat olfactory bulb. J. Neurophysiol. 91, 2532-2540.

Nagayama, S., Homma, R., and Imamura, F. (2014). Neuronal organization of olfactory bulb circuits. Front. Neural Circuits 8, 98.

Najac, M., Jan, D. de Saint, Reguero, L., Grandes, P., and Charpak, S. (2011). Monosynaptic and polysynaptic feed-forward inputs to mitral cells from olfactory sensory neurons. J. Neurosci. 31, 8722-8729.

Nakamura, T., and Gold, G.H. (1987). A cyclic nucleotide-gated conductance in olfactory cilia. Nature 325, 442.

Nakamuta, S., Nakamuta, N., and Taniguchi, K. (2011). Distinct axonal projections from two types of olfactory receptor neurons in the middle chamber epithelium of Xenopus laevis. Cell Tissue Res. 346, 27.

Nakashima, A., Takeuchi, H., Imai, T., Saito, H., Kiyonari, H., Abe, T., Chen, M., Weinstein, L.S., Yu, C.R., Storm, D.R., et al. (2013). Agonist-independent GPCR activity regulates anterior-posterior targeting of olfactory sensory neurons. Cell 154, 1314-1325.

Nakashima, A., Ihara, N., Shigeta, M., Kiyonari, H., Ikegaya, Y., and Takeuchi, H. (2019). Structured spike series specify gene expression patterns for olfactory circuit formation. Science (80-. ). 365, eaaw5030.

Nassi, J.J., Cepko, C.L., Born, R.T., and Beier, K.T. (2015). Neuroanatomy goes viral! Front. Neuroanat. 9, 80.

Neumann, E., Kakorin, S., and Tœnsing, K. (1999). Fundamentals of electroporative delivery of drugs and genes. Bioelectrochemistry Bioenerg. 48, 3-16.

Nezlin, L.P., and Schild, D. (2000). Structure of the olfactory bulb in tadpoles of Xenopus laevis. Cell Tissue Res. 302, 21-29.

Nezlin, L.P., and Schild, D. (2005). Individual olfactory sensory neurons project into more than one glomerulus in Xenopus laevis tadpole olfactory bulb. J. Comp. Neurol. 481, 233239.

Nezlin, L.P., Heermann, S., Schild, D., and Rössler, W. (2003). Organization of glomeruli in the main olfactory bulb of Xenopus laevis tadpoles. J. Comp. Neurol. 464, 257-268. 
Ngai, J., Dowling, M.M., Buck, L., Axel, R., and Chess, A. (1993). The Family of genes encoding odorant receptors in the channel catfish. Cell 72, 657-666.

Nieuwkoop, P.D., and Faber, J. (1994). Normal Table of Xenopus laevis (Daudin). In New York, NY: Garland Publishing Inc, p. 252.

Niimura, Y., and Nei, M. (2005a). Comparative evolutionary analysis of olfactory receptor gene clusters between humans and mice. Gene 346, 13-21.

Niimura, Y., and Nei, M. (2005b). Evolutionary dynamics of olfactory receptor genes in fishes and tetrapods. 102, 6039-6044.

Niimura, Y., and Nei, M. (2007). Extensive gains and losses of olfactory receptor genes in mammalian evolution. PLoS One 2, e708.

Nishizumi, H., Miyashita, A., Inoue, N., Inokuchi, K., Aoki, M., and Sakano, H. (2019). Primary dendrites of mitral cells synapse unto neighboring glomeruli independent of their odorant receptor identity. Commun. Biol. 2, 14.

Nodari, F., Hsu, F.-F., Fu, X., Holekamp, T.F., Kao, L.-F., Turk, J., and Holy, T.E. (2008). Sulfated steroids as natural ligands of mouse pheromone-sensing neurons. J. Neurosci. 28, 6407-6418.

Oka, Y. (1983). Golgi, electron-microscopic and combined golgi-electron-microscopic studies of the mitral cells in the goldfish olfactory bulb. Neuroscience $8,723-742$.

Oka, Y., Saraiva, L.R., and Korsching, S.I. (2012). Crypt neurons express a single v1rrelated ORA gene. Chem. Senses 37, 219-227.

Orona, E., Rainer, E.C., and Scott, J.W. (1984). Dendritic and axonal organization of mitral and tufted cells in the rat olfactory bulb. J. Comp. Neurol. 226, 346-356.

Otazu, G.H., Chae, H., Davis, M.B., and Albeanu, D.F. (2015). Cortical feedback decorrelates olfactory bulb output in awake mice. Neuron 86, 1461-1477.

Ottoson, D. (1956). Analysis of the electrical activity of the olfactory epithelium. Acta Physiol. Scand. 35, 1-83.

Pace, U., Hanski, E., Salomon, Y., and Lancet, D. (1985). Odorant-sensitive adenylate cyclase may mediate olfactory reception. Nature 316, 255.

Panzeri, S., Brunel, N., Logothetis, N.K., and Kayser, C. (2010). Sensory neural codes using multiplexed temporal scales. Trends Neurosci. 33, 111-120.

Paredes, R.M., Etzler, J.C., Watts, L.T., and Lechleiter, J.D. (2009). Chemical Calcium Indicators. Methods 46, 143-151.

Peticolas, W.L., Goldsborough, J.P., and Rieckhoff, K.E. (1963). Double photon excitation 
in organic crystals. Phys. Rev. Lett. 10, 43-45.

Pfister, P., and Rodriguez, I. (2005). Olfactory expression of a single and highly variable V1r pheromone receptor-like gene in fish species. PNAS 102, 5489-5494.

Pinching, A.J., and Powell, T.P.S. (1971a). The neuron types of the glomerular layer of the olfactory bulb. J. Cell Sci. 9, 305-345.

Pinching, A.J., and Powell, T.P.S. (1971b). The neuropil of the periglomerular region of the olfactory bulb. J. Cell Sci. 9, 347-377.

Pnevmatikakis, E.A., and Giovannucci, A. (2017). NoRMCorre: An online algorithm for piecewise rigid motion correction of calcium imaging data. J. Neurosci. Methods 291, 8394.

Pnevmatikakis, E.A., Gao, Y., Soudry, D., Pfau, D., Lacefield, C., Poskanzer, K., Bruno, R., Yuste, R., and Paninski, L. (2014). A structured matrix factorization framework for large scale calcium imaging data analysis. ArXiv Prepr. ArXiv1409.2903.

Pnevmatikakis, E.A., Soudry, D., Gao, Y., Machado, T.A., Merel, J., Pfau, D., Reardon, T., Mu, Y., Lacefield, C., Yang, W., et al. (2017). Simultaneous denoising, deconvolution, and demixing of calcium imaging data. Neuron 89, 285-299.

Poplawsky, A.J., and Kim, S.-G. (2014). Layer-dependent BOLD and CBV-weighted fMRI responses in the rat olfactory bulb. Neuroimage 91, 237-251.

Pouget, A., Dayan, P., and Zemel, R. (2000). Information Processing with population codes. Nat. Rev. Neurosci. 1, 125.

Price, J.L., and Powell, T.P.S. (1970). The morphology of the granule cells of the olfactory bulb. J. Cell Sci. 7, 91-123.

Del Punta, K., Puche, A., Adams, N.C., Rodriguez, I., and Mombaerts, P. (2002). A divergent pattern of sensory axonal projections is rendered convergent by second-order neurons in the accessory olfactory bulb. Neuron 35, 1057-1066.

Quaglino, E., Giustetto, M., Panzanelli, P., Cantino, D., Fasolo, A., Sassoe, M., Anatomia, D., Legale, M., Torino, I.-, Animale, B., et al. (1999). Immunocytochemical localization of glutamate and $\mathrm{y}$-aminobutyric acid in the accessory olfactory bulb of the rat. J. Comp. Neurol. 408, 61-72.

Rajan, R. (2006). Rats smell in stereo. Science (80-. ). 311, 666-670.

Rall, W., Shepherd, G.M., Reese, S., and Brightman, M.W. (1966). Dendrodendritic synaptic pathway for inhibition in the olfactory bulb. Exp. Neurol. 14, 44-56.

Rebello, M.R., Kandukuru, P., and Verhagen, J. V. (2015). Direct behavioral and 
neurophysiological evidence for retronasal olfaction in mice. PLoS One 10, e0117218.

Reeber, S.L., Gebre, S. a., and Sillitoe, R. V. (2011). Fluorescence mapping of afferent topography in three dimensions. Brain Struct. Funct. 216, 159-169.

Reiner, A., Veenman, C.L., Medina, L., Jiao, Y., Del Mar, N., and Honig, M. (2000). Pathway tracing using biotinylated dextran amines Pathway tracing using biotinylated dextran amines. J. Neurosci. 103, 23-37.

Ressler, K.J., Sullivan, S.L., and Buck, L.B. (1993). A zonal organization of odorant receptor gene expression in the olfactory epithelium. Cell 73, 597-609.

Ressler, K.J., Sullivan, S.L., and Buck, L.B. (1994). Information coding in the olfactory system: evidence for a stereotyped and highly organized epitope map in the olfactory bulb. Cell 79, 1245-1255.

Restrepo, D., Boekhoff, I., and Breer, H. (1993). Rapid kinetic measurements of second messenger formation in olfactory cilia from channel catfish. Am. J. Physiol. Physiol. 264, c906-C911.

Rivière, S., Challet, L., Fluegge, D., Spehr, M., and Rodriguez, I. (2009). Formyl peptide receptor-like proteins are a novel family of vomeronasal chemosensors. Nature 459, 574.

Rodolfo-Masera, T. (1943). Su l'esistenza di un particolare organo olfacttivo nel setto nasale della cavia e di altri roditori. Arch Ital Anat Embryol 157-212.

Rodriguez, I., Feinstein, P., and Mombaerts, P. (1999). Variable patterns of axonal projections of sensory neurons in the mouse vomeronasal system. Cell 97, 199-208.

Rünnenburger, K., Breer, H., and Boekhoff, I. (2002). Selective G protein $\beta \gamma$-subunit compositions mediate phospholipase $\mathrm{C}$ activation in the vomeronasal organ. Eur. J. Cell Biol. 81, 539-547.

Ryba, N.J.P., and Tirindelli, R. (1995). A novel GTP binding protein y-subunit, Gy8, Is expressed during neurogenesis in the olfactory and vomeronasal neuroepithelia.pdf. J. Biol. Chem. 270, 6757-6767.

Ryba, N.J.P., and Tirindelli, R. (1997). A new multigene family of putative pheromone receptors. Neuron 19, 371-379.

Ryerson, W.G., and Deban, S.M. (2010). Buccal pumping mechanics of Xenopus laevis tadpoles : effects of biotic and abiotic factors. J. Exp. Biol. 213, 2444-2452.

De Saint Jan, D., Hirnet, D., Westbrook, G.L., and Charpak, S. (2009). External tufted cells drive the output of olfactory bulb glomeruli. J. Neurosci. 29, 2043-2052.

Salin, P.-A., Lledo, P.-M., Vincent, J.-D., and Charpak, S. (2000). Dendritic glutamate 
autoreceptors modulate signal processing in rat mitral cells. J. Neurophysiol. $85,1275-$ 1282.

Sansone, A., Hassenklöver, T., Syed, A.S., Korsching, S.I., and Manzini, I. (2014a).

Phospholipase $\mathrm{C}$ and diacylglycerol mediate olfactory responses to amino acids in the main olfactory epithelium of an amphibian. PLoS One 9, e87721.

Sansone, A., Syed, A.S., Tantalaki, E., Korsching, S.I., and Manzini, I. (2014b). Trpc2 is expressed in two olfactory subsystems, the main and the vomeronasal system of larval Xenopus laevis. J. Exp. Biol. 217, 2235-2238.

Sansone, A., Hassenklöver, T., Offner, T., Fu, X., Holy, T.E., and Manzini, I. (2015). Dual processing of sulfated steroids in the olfactory system of an anuran amphibian. Front. Cell. Neurosci. 9, 373.

Saraiva, L.R., and Korsching, S.I. (2007). A novel olfactory receptor gene family in teleost fish. Genome Res. 17, 1448-1457.

Sathyanesan, A., Feijoo, A.A., Mehta, S.T., Nimarko, A.F., and Lin, W. (2013). Expression profile of $\mathrm{G}$-protein $\beta$ y subunit gene transcripts in the mouse olfactory sensory epithelia. Front. Cell. Neurosci. 7, 84.

Sato, Y., Miyasaka, N., and Yoshihara, Y. (2005). Mutually exclusive glomerular innervation by two distinct types of olfactory sensory neurons revealed in transgenic zebrafish. J. Neurosci. 25, 4889-4897.

Sato, Y., Miyasaka, N., and Yoshihara, Y. (2007). Hierarchical regulation of odorant receptor gene choice and subsequent axonal projection of olfactory sensory neurons in zebrafish. J. Neurosci. 27, 1606-1615.

Scalia, F., and Winans, S.S. (1975). The differential projections of the olfactory bulb and accessory olfactory bulb in mammals. J Comp Neurol 161, 31-56.

Scalia, F., Gallousis, G., and Roca, S. (1991). A note on the organization of the amphibian olfactory bulb. J. Comp. Neurol. 305, 435-442.

Schaefer, A.T., and Margrie, T.W. (2012). Psychophysical properties of odor processing can be quantitatively described by relative action potential latency patterns in mitral and tufted cells. Front. Syst. Neurosci. 6, 30.

Schafer, J.R., Kida, I., Xu, F., Rothman, D.L., and Hyder, F. (2006). Reproducibility of odor maps by fMRI in rodents. Neuroimage 31, 1238-1246.

Schild, D., and Manzini, I. (2004). Cascades of response vectors of olfactory receptor neurons in Xenopus laevis tadpoles. Eur. J. Neurosci. 20, 2111-2123.

Schild, D., and Restrepo, D. (1998). Transduction mechanisms in vertebrate olfactory 
receptor cells. Physiol. Rev. 78, 429-466.

Schindelin, J., Arganda-Carrera, I., Frise, E., Verena, K., Mark, L., Tobias, P., Stephan, P., Curtis, R., Stephan, S., Benjamin, S., et al. (2012). Fiji - an Open platform for biological image analysis. Nat. Methods 9, 676.

Schneider, S.P., and Macrides, F. (1978). Laminar distributions of interneurons in the main olfactory bulb of the adult hamster. Brain Res. Bull. 3, 73-82.

Schoppa, N.E., and Urban, N.N. (2003). Dendritic processing within olfactory bulb circuits. Trends Neurosci. 26, 501-506.

Schoppa, N.E., and Westbrook, G.L. (2001). Glomerulus-specific synchronization of mitral cells in the olfactory bulb. Neuron 31,639-651.

Schultze, M.J.S. (1856). Über die Endigungsweise des Geruchsnerven und die Epithelialgebilde der Nasenschleimhaut. Verhandl. Akad.Wissensch. Berlin 21, $504-514$.

Schwartz-Levey, M., Cinelli, A.R., and Kauer, J.S. (1992). Intracellular injection of vital dyes into single cells in the salamander olfactory epithelium. Neurosci. Lett. 140, 265-269.

Seale, D.B., Hoff, K., and Wassersug, R. (1982). Xenopus laevis larvae (Amphibia , Anura) as model suspension feeders. Hydrobiologia 87, 161-169.

Serizawa, S., Ishii, T., Nakatani, H., Tsuboi, A., Nagawa, F., Asano, M., Sudo, K., Sakagami, J., Sakano, H., ljiri, T., et al. (2000). Mutually exclusive expression of odorant receptor transgenes. Nat. Neurosci. 3, 687.

Shao, X., Lakhina, V., Dang, P., Cheng, R.P., Marcaccio, C.L., and Raper, J.A. (2017). Olfactory sensory axons target specific protoglomeruli in the olfactory bulb of zebrafish. Neural Dev. 12, 18.

Sharp, F.R., Kauer, J.S., and Shepherd, G.M. (1975). Local sites of activity-related glucose metabolism in rat olfactory bulb during olfactory stimulation. Brain Res. 98, 596-600.

Shepherd, G.M., Chen, W.R., Willhite, D., Migliore, M., and Greer, C.A. (2007). The olfactory granule cell: From classical enigma to central role in olfactory processing. Brain Res. Rev. 55, 373-382.

Shi, L., and Javitch, J.A. (2002). The binding site of aminergic G protein-coupled receptors: The transmembrane segments and second extracellular loop. Annu Rev. Pharmacol Toxicol 42, 437-467.

Shi, P., and Zhang, J. (2007). Comparative genomic analysis identifies an evolutionary shift of vomeronasal receptor gene repertoires in the vertebrate transition from water to land.

Genome Res. 17, 166-174. 
Silva, L., and Antunes, A. (2017). Vomeronasal receptors in vertebrates and the evolution of pheromone detection. Annu. Rev. Anim. Biosci. 5, 353-370.

Silvotti, L., Moiani, A., Gatti, R., and Tirindelli, R. (2007). Combinatorial co-expression of pheromone receptors, V2Rs. J. Neurochem. 103, 1753-1763.

Speca, D.J., Lin, D.M., Sorensen, P.W., Isacoff, E.Y., Ngai, J., and Dittman, A.H. (1999). Functional identification of a goldfish odorant receptor. Neuron 23, 487-498.

Spehr, M., and Munger, S.D. (2009). Olfactory receptors: GPCRs and beyond. J. Neurochem. 109, 1570-1583.

Spehr, M., Hatt, H., and Wetzel, C.H. (2002). Arachidonic acid plays a role in rat vomeronasal signal transduction. J. Neurosci. 22, 8429-8437.

Spence, D.E., Kean, P.N., and Sibbett, W. (1991). 60-fsec pulse generation from a selfmode-locked $\mathrm{Ti}$ : sapphire laser. Opt. Lett. 16, 42-44.

Spors, H., and Grinvald, A. (2002). Spatio-temporal dynamics of odor representations in the mammalian olfactory bulb. Neuron 34, 301-315.

Spors, H., Wachowiak, M., Cohen, L.B., and Friedrich, R.W. (2006). Temporal dynamics and latency patterns of receptor neuron input to the olfactory bulb. J. Neurosci. 26, 12471259.

Sprague, J., Bayraktaroglu, L., Clements, D., Conlin, T., Fashena, D., Frazer, K., Haendel, M., Howe, D.G., Mani, P., Ramachandran, S., et al. (2006). The zebrafish information network: the zebrafish model organism database. Nucleic Acids Res. 34, D581-D585.

Stokes, G.G. (1852). On the change of refrangibility of light. Philos. Trans. R. Soc. London 142, 463-562.

Storace, D.A., and Cohen, L.B. (2017). Measuring the olfactory bulb input-output transformation reveals a contribution to the perception of odorant concentration invariance. Nat. Commun. 8, 81.

Svoboda, K., Denk, W., Kleinfeld, D., and Tank, D.W. (1997). In vivo dendritic calcium dynamics in neocortical pyramidal neurons. Nature 385, 161.

Syed, A.S., Sansone, A., Nadler, W., Manzini, I., and Korsching, S.I. (2013). Ancestral amphibian v2r s are expressed in the main olfactory epithelium. PNAS 110, 7714-7719.

Syed, A.S., Sansone, A., Röner, S., Bozorg Nia, S., Manzini, I., and Korsching, S.I. (2015). Different expression domains for two closely related amphibian TAARs generate a bimodal distribution similar to neuronal responses to amine odors. Sci. Rep. 5, 13935.

Syed, A.S., Sansone, A., Hassenklöver, T., Manzini, I., and Korsching, S.I. (2017). 
Coordinated shift of olfactory amino acid responses and V2R expression to an amphibian water nose during metamorphosis. Cell. Mol. Life Sci. 74, 1711-1719.

Takahashi, H., Yoshihara, S., Nishizumi, H., and Tsuboi, A. (2010). Neuropilin-2 is required for the proper targeting of ventral glomeruli in the mouse olfactory bulb. Mol. Cell. Neurosci. 44, 233-245.

Takami, S., and Graziadei, P.P.C. (1991). Light microscopic golgi study of mitral/tufted cells in the accessory olfactory bulb of the adult rat. J. Comp. Neurol. 311, 65-83.

Taniguchi, M., and Kaba, H. (2001). Properties of reciprocal synapses in the mouse accessory olfactory bulb. Neuroscience 108, 365-370.

Terni, B., Pacciolla, P., Masanas, H., Gorostiza, P., and Llobet, A. (2017). Tight temporal coupling between synaptic rewiring of olfactory glomeruli and the emergence of odorguided behavior in Xenopus tadpoles. J. Comp. Neurol. 525, 3769-3783.

Thommesen, G. (1983). Morphology, distribution, and specificity of olfactory receptor cells in salmonid fishes. Acta Physiol. Scand. 117, $241-249$.

Tolokh, I.I., Fu, X., and Holy, T.E. (2013). Reliable sex and strain discrimination in the mouse vomeronasal organ and accessory olfactory bulb. J. Neurosci. 33, 13903-13913.

Treloar, H.B., Purcell, A.L., and Greer, C.A. (1999). Glomerular formation in the developing rat olfactory bulb. J. Comp. Neurol. 413, 289-304.

Trotier, D., and Døving, K.B. (1998). Anatomical description of a new organ in the nose of domesticated animals by Ludvig Jacobson. Chem Senses 23, 743-754.

Tsien, R.Y. (1981). A non disruptive technique for loading calcium buffers and indicators into cells.pdf. Nature 290, 527.

Tsien, R.Y. (2005). Building and breeding molecules to spy on cells and tumors. FEBS Lett. $579,927-932$.

Tsuboi, A., Miyazaki, T., Imai, T., and Sakano, H. (2006). Olfactory sensory neurons expressing class I odorant receptors converge their axons on an antero-dorsal domain of the olfactory bulb in the mouse. Eur. J. Neurosci. 23, 1436-1444.

Tsuriel, S., Gudes, S., Draft, R.W., Binshtok, A.M., and Lichtman, J.W. (2015). Multispectral labeling technique to map many neighboring axonal projections in the same tissue. Nat.

Methods 12, 547.

Turaga, D., and Holy, T.E. (2012). Organization of vomeronasal sensory coding revealed by fast volumetric calcium imaging. J. Neurosci. 32, 1612-1621.

Uchida, N., and Mainen, Z.F. (2003). Speed and accuracy of olfactory discrimination in the 
rat. Nat. Neurosci. 6, 1224-1229.

Uchida, N., Takahashi, Y.K., Tanifuji, M., and Mori, K. (2000). Odor maps in the mammalian olfactory bulb: Domain organization and odorant structural features. Nat. Neurosci. 3, 1035.

Untiet, V., Moeller, L.M., Ibarra-Soria, X., Sánchez-Andrade, G., Stricker, M., Neuhaus, E.M., Logan, D.W., Gensch, T., and Spehr, M. (2016). Elevated cytosolic Cl concentrations in dendritic knobs of mouse vomeronasal sensory neurons. Chem. Senses 41, 669-676.

Urban, N.N., and Castro, J.B. (2005). Tuft calcium spikes in accessory olfactory bulb mitral cells. J. Neurosci. 25, 5024-5028.

Urban, N.N., and Sakmann, B. (2002). Reciprocal intraglomerular excitation and intra- and interglomerular lateral inhibition between mouse olfactory bulb mitral cells. J. Physiol. 542, 355-367.

Vassar, R., Ngai, J., and Axel, R. (1993). Spatial segregation of odorant receptor expression in the mammalian olfactory epithelium. Cell 74, 309-318.

Vassar, R., Chao, S.K., Sitcheran, R., Nuñez, J.M., Vosshall, L.B., and Axel, R. (1994). Topographic organization of sensory projections to the olfactory bulb. Cell 79, 981-991.

Victor, J.D. (2000). How the brain uses time to represent and process visual information. 886, 33-46.

Wachowiak, M., and Cohen, L.B. (2001). Representation of odorants by receptor neuron input to the mouse olfactory bulb. Neuron 32, 723-735.

Wachowiak, M., and Shipley, M.T. (2006). Coding and synaptic processing of sensory information in the glomerular layer of the olfactory bulb. Semin. Cell Dev. Biol. 17, 411-423. Wachowiak, M., Denk, W., and Friedrich, R.W. (2004). Functional organization of sensory input to the olfactory bulb glomerulus analyzed by two-photon calcium imaging. PNAS 101, 9097-9102.

Wachowiak, M., Economo, M.N., Díaz-quesada, M., Brunert, D., Wesson, D.W., White, J.A., and Rothermel, M. (2013). Optical dissection of odor information processing in vivo using GCaMPs expressed in specified cell types of the olfactory bulb. J. Neurosci. 33, 5285-5300.

Wagner, S., Gresser, A.L., Torello, A.T., and Dulac, C. (2006). A multireceptor genetic approach uncovers an ordered integration of VNO sensory inputs in the accessory olfactory bulb. Neuron 50, 697-709.

Wakisaka, N., Miyasaka, N., Koide, T., Masuda, M., Hiraki-Kajiyama, T., and Yoshihara, Y. (2017). An adenosine receptor for olfaction in fish. Curr. Biol. 27, 1437-1447.e4. 
Wanner, A.A., Genoud, C., Masudi, T., Siksou, L., and Friedrich, R.W. (2016). Dense EMbased reconstruction of the interglomerular projectome in the zebrafish olfactory bulb. Nat. Neurosci. 19, 816.

Weiss, L., Offner, T., Hassenklöver, T., and Manzini, I. (2018). Dye electroporation and imaging of calcium signaling. In Xenopus: Methods and Protocols, Methods in Molecular Biology, pp. 217-231.

Weiss, L., Jungblut, L.D., Pozzi, A.G., Zielinski, B.S., Connell, L.A.O., Hassenklöver, T., and Manzini, I. (2019). Multi-glomerular projection of single olfactory receptor neurons is conserved among amphibians. BioRxiv 788133.

Whitesell, J.D., Sorensen, K.A., Jarvie, B.C., Hentges, S.T., and Schoppa, N.E. (2013). Interglomerular lateral inhibition targeted onexternal tufted cells in the olfactory bulb. $\mathrm{J}$. Neurosci. 33, 1552-1563.

Willhite, D.C., Nguyen, K.T., Masurkar, A. V., Greer, C.A., Shepherd, G.M., and Chen, W.R. (2006). Viral tracing identifies distributed columnar organization in the olfactory bulb. PNAS 103, 12592-12597.

Wilson, K.C.P., and Raisman, G. (1980). Age-related changes in the neurosensory spithelium of the mouse vomeronasal organ: Extended period of post-natal growth in size and evidence for rapid cell turnover in the adult. Brain Res. 185, 103-113.

Wilson, T., and Sheppard, C. (1984). Theory and practice of scanning optical microscopy. Theory Pract. Scanning Opt. Microsc. 180, Londo.

Wong, S.T., Trinh, K., Hacker, B., Chan, G.C.K., Lowe, G., Gaggar, A., Xia, Z., Gold, G.H., and Storm, D.R. (2000). Disruption of the type III adenylyl cyclase gene leads to peripheral and behavioral anosmia in transgenic mice. Neuron 27, 487-497.

Woodley, S. (2014). Hormones and behavior chemosignals, hormones, and amphibian reproduction. Horm. Behav. 68, 8-13.

Wu, Y., Tirindelli, R., and Ryba, N.J.P. (1996). Evidence for different chemosensory signal transduction pathways in olfactory and vomeronasal neurons. Biochem. Biophys. Res. Commun. 220, 900-904.

Xu, F., Liu, N., Kida, I., Rothman, D.L., Hyder, F., and Shepherd, G.M. (2003). Odor maps of aldehydes and esters revealed by functional MRI in the glomerular layer of the mouse olfactory bulb. PNAS 100, 11029-11034.

Xu, P.S., Lee, D., and Holy, T.E. (2016). Experience-dependent plasticity drives individual differences in pheromone-sensing neurons. Neuron 91, 878-892.

Yabuki, Y., Koide, T., Miyasaka, N., Wakisaka, N., Masuda, M., Ohkura, M., Nakai, J., 
Tsuge, K., Tsuchiya, S., Sugimoto, Y., et al. (2016). Olfactory receptor for prostaglandin F2 $\alpha$ mediates male fish courtship behavior. Nat. Neurosci. 19, 897-904.

Yang, C., and Delay, R.J. (2010). Calcium-activated chloride current amplifies the response to urine in mouse vomeronasal sensory neurons. J. Gen. Physiol. 135, 3-13.

Yang, W., and Yuste, R. (2017). In vivo imaging of neural activity. Nat. Methods 14, 349. Yoles-Frenkel, M., Kahan, A., and Ben-shaul, Y. (2018). Temporal response properties of accessory olfactory bulb neurons : Limitations and opportunities for decoding. J. Neurosci. 38, 4957-4976.

Yonekura, J., and Yokoi, M. (2008). Conditional genetic labeling of mitral cells of the mouse accessory olfactory bulb to visualize the organization of their apical dendritic tufts. Mol. Cell. Neurosci. 37, 708-718.

Yoshihara, Y. (2002). Visualizing selective neural pathways with WGA transgene: combination of neuroanatomy with gene technology. Neurosci. Res. 44, 133-140.

Yoshihara, Y., Mizuno, T., Nakahira, M., Kawasaki, M., Watanabe, Y., Kagamiyama, H., Jishage, K., Ueda, O., Suzuki, H., Tabuchi, K., et al. (1999). A genetic approach to visualization of multisynaptic neural pathways using plant lectin transgene. Neuron 22, 3341.

Young, J.M., and Trask, B.J. (2007). V2R gene families degenerated in primates, dog and cow, but expanded in opossum. Trends Genet. 23, 212-215.

Yuan, Q., and Knöpfel, T. (2006). Olfactory nerve stimulation-induced calcium signaling in the mitral cell distal dendritic tuft. J. Neurophysiol. 95, 2417-2426.

Zapiec, B., and Mombaerts, P. (2015). Multiplex assessment of the positions of odorant receptor-specific glomeruli in the mouse olfactory bulb by serial two-photon tomography. PNAS 112, E5873-E5882.

Zhang, C., and Restrepo, D. (2002). Expression of connexin 45 in the olfactory system. Brain Res. 929, 37-47.

Zhang, X., and Firestein, S. (2002). The olfactory receptor gene superfamily of the mouse. Nat. Neurosci. 5, 124.

Zhang, L., Huang, Y., and Hu, B. (2016). Olfactory experiences dynamically regulate plasticity of dendritic spines in granule cells of Xenopus tadpoles in vivo. Sci. Rep. 6 , 35009.

Zhang, X., Rodriguez, I., Mombaerts, P., and Firestein, S. (2004). Odorant and vomeronasal receptor genes in two mouse genome assemblies. Genomics 83, 802-811. 
Zou, D., Chesler, A.T., Le Pichon, C.E., Kuznetsov, A., Pei, X., and Hwang, E.L. (2007).

Absence of adenylyl cyclase 3 perturbs peripheral olfactory projections in mice. J. Neurosci.

27, 6675-6683. 